\title{
A Thermoelastic Hydraulic Fracture Design Tool for Geothermal Reservoir Development
}

Final Report Submitted By:

\author{
Ahmad Ghassemi \\ DEPARTMENT OF GEOLOGY \& GEOLOGICAL ENGINEERING \\ UNIVERSITY OF NORTH DAKOTA \\ GRAND FORKS, ND \\ June 2003
}

Prepared for the U.S. DOE Assistant Secretary for Energy Efficiency \& Renewable Energy Under DOE Idaho Operations Office

Financial Assistance Award DE-FG07-99ID13855 


\section{Contents}

1 Introduction $\quad 1$

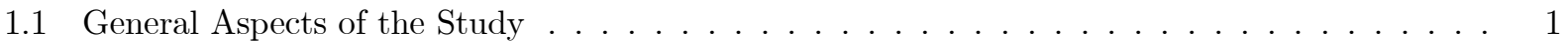

1.2 Issues \& Scope . . . . . . . . . . . . . . . . . . . . . . . . . . . . . 1

2 Hydraulic Fracturing in Geothermal Reservoirs: Fundamental Concepts \& Mathematical Framework

2.1 Fracture Initiation $\ldots \ldots \ldots \ldots \ldots \ldots \ldots \ldots \ldots \ldots$

2.1.1 Hydraulic fracture initiation . . . . . . . . . . . . . . . . . . . . 4

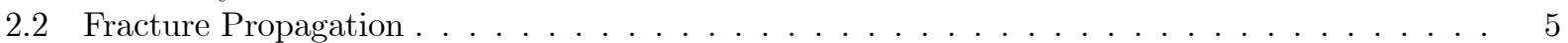

2.2 .1 Mode-I or opening mode . . . . . . . . . . . . . . . . . . . . . . . . 9

2.2 .2 Mode-II or sliding mode . . . . . . . . . . . . . . . . . . . . . . . . . . . . . 9

2.2 .3 Mode-III or tearing mode . . . . . . . . . . . . . . . . . . . . . . . . . 9

2.3 Fluid Flow in a Fracture . . . . . . . . . . . . . . . . . . . . . . . . . . . . . 13

2.4 Fluid Diffusion into the Formation (Leak-off) $\ldots \ldots \ldots \ldots \ldots \ldots \ldots$

2.5 Hear Transfer \& Heat Flow Model Development . . . . . . . . . . . . . . . . . . . . . 18

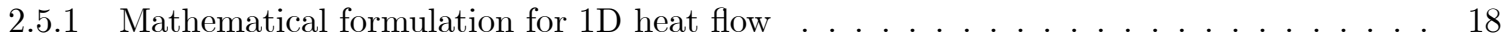

2.5.2 Mathematical model for 2D heat flow . . . . . . . . . . . . . . . . . . 21

2.5.3 Mathematical model for 3D heat flow . . . . . . . . . . . . . . . . . . . 22

2.6 Coupled Poro-Thermoelastic Processes . . . . . . . . . . . . . . . . . . . . . . . . . . . 24

3 Mathematical Methods \& Numerical Algorithms 28

3.1 Poro-Thermoelastic Boundary Element Method . . . . . . . . . . . . . . . . . . 28

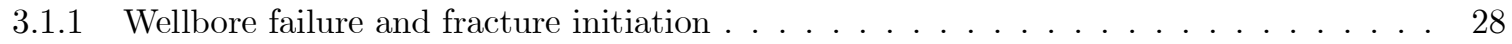

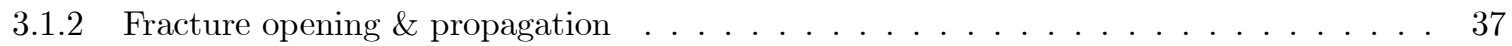

3.2 Modeling Fracture Propagation . . . . . . . . . . . . . . . . . . . 50

3.2 .1 Complex variable integral equation method . . . . . . . . . . . . . . . 50

4 Examples \& Applications $\quad 55$

4.1 Fracture Propagation from a Wellbore: An Isothermal Fracture Mechanics Analysis . . . . . 55

4.1 .1 Influence of various parameters on propagation . . . . . . . . . . . . . 56

4.1 .2 Hydraulic fracture propagation near a fault $\ldots \ldots \ldots \ldots \ldots$

4.1 .3 Implications for geothermal reservoir stimulation . . . . . . . . . . . . . . . . 81

4.2 Graphics User Interface . . . . . . . . . . . . . . . . . . . . . . . . . . . . 82

4.2 .1 General concepts \& characteristics . . . . . . . . . . . . . . . . . . . . 82

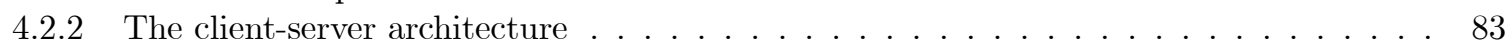

4.2 .3 En echelon fracture . . . . . . . . . . . . . . . . . . . . . . . . 84

4.2 .4 Fracture propagation from a wellbore . . . . . . . . . . . . . . . 84

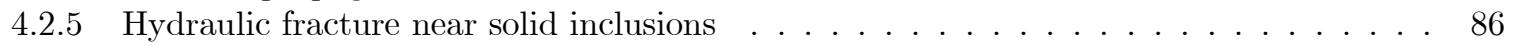

4.3 Heat Extraction . . . . . . . . . . . . . . . . . . . . . . . . . . 86

4.3 .1 Solution of the general $1 \mathrm{D}$ model $\ldots \ldots \ldots \ldots \ldots$

4.3.2 Integral formulation \& solution of the $2 \mathrm{D}$ model $\ldots \ldots \ldots \ldots$

4.3.3 Integral formulation \& solution for the $3 \mathrm{D}$ model $\ldots \ldots \ldots \ldots$

5 Conclusions \& Future Research $\quad 120$

5.1 Heat Extraction \& Thermoelastic Effects . . . . . . . . . . . . . . . . . . . . . . 120

5.2 Wellbore Failure and Fracture Initiation; Porothermoelastic Effects . . . . . . . . . . . . . 121

5.3 Fracture Propagation . . . . . . . . . . . . . . . . . . . . . . . . . . 121

5.4 Graphics User Interface . . . . . . . . . . . . . . . . . . . . . . . . . . . . 122

5.5 Practical Guidelines for Enhanced Geothermal Systems _ . . . . . . . . . . . . . . 122 


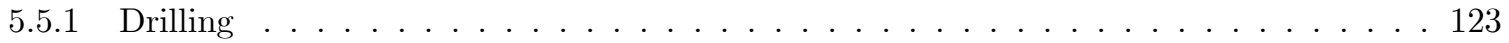

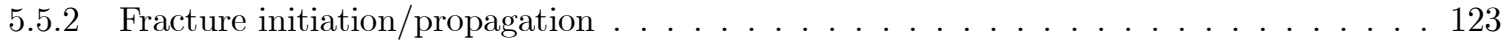

5.5 .3 Fracturing of naturally-fractured reservoirs $\ldots \ldots \ldots \ldots \ldots \ldots \ldots \ldots \ldots$

5.5 .4 Influence of cold fluid injection . . . . . . . . . . . . . . . . . . 123

5.6 Future Work . . . . . . . . . . . . . . . . . . . . . . . . . . . . 124

6 Appendix A $\quad 134$

6.1 Mathematical Formulation and Numerical Aspects of GeoFrac . . . . . . . . . . . . . . . 134

6.1.1 Basic integral equation for a plane without a circular hole . . . . . . . . . . . . . 134

6.1 .2 Basic integral equation for a plane with a circular hole . . . . . . . . . . . . . . 134

6.1 .3 Calculation of SIFs . . . . . . . . . . . . . . . . . . . 136

6.1.4 Approximation of the crack path at each stage of propagation . . . . . . . . . . . 139

6.2 Numerical Algorithm for Crack Propagation . . . . . . . . . . . . . . . . . . . . 141

7 Appendix B $\quad 142$

7.1 Graphics User Interface $(\mathrm{GUI}) \quad \ldots \ldots \ldots \ldots \ldots$

7.1 .1 Input procedures: . . . . . . . . . . . . . . . . . . . . . 145

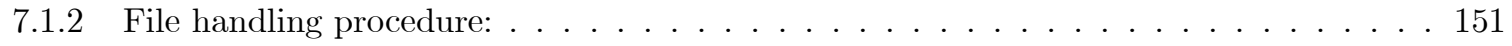

7.1 .3 Simulation output . . . . . . . . . . . . . . . . . . . . 152

7.1 .4 Real time output . . . . . . . . . . . . . . . . . . . . . 158

7.1 .5 Current limitations . . . . . . . . . . . . . . . . . . . . . . . . 164

8 Appendix C $\quad 166$

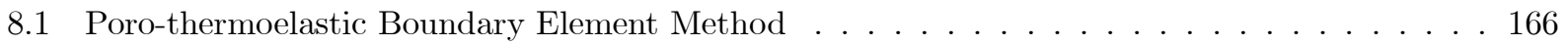

$9 \begin{array}{ll}168 \\ \end{array}$

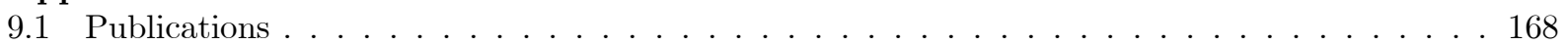




\section{List of Figures}

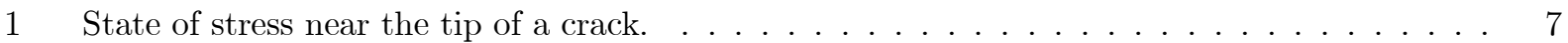

2 A sketch of three fundamental modes of fracture . . . . . . . . . . . . . . . . 8

3 Problem geometry for an inclined crack. . . . . . . . . . . . . . . . . . . 11

4 Crack-tip closure resulting from Barenblatt's $3 r d$ postulate . . . . . . . . . . . . . . . 12

5 Paralle plate model for flow in a fracture . . . . . . . . . . . . . . . . . 15

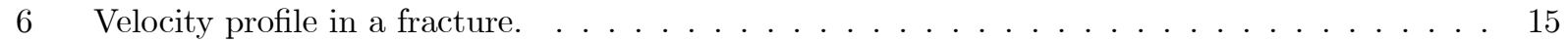

$7 \quad$ Idealized view of heat extraction from a hot dry rock reservoir, 1D model. $\ldots \ldots \ldots$

8 Mathematical model for heat extraction from a fracture. . . . . . . . . . . . . . . . 20

9 Geometry of two-dimensional heat extraction from a reservoir. . . . . . . . . . . . . 21

10 Heat extraction from a plane fracture. . . . . . . . . . . . . . . . . . . 23

11 Problem geometry and boundary conditions for a circular borehole in an infinite porous medium. 28

12 Temperature distribution around the wellbore. . . . . . . . . . . . . . . . 29

13 Cooling induced pore pressure around the wellbore. . . . . . . . . . . . . . . . . . 30

14 Cooling induced radial stress around the wellbore. . . . . . . . . . . . . . . . . . 31

15 Cooling induced tangential stress around the wellbore. . . . . . . . . . . . . . . . . 32

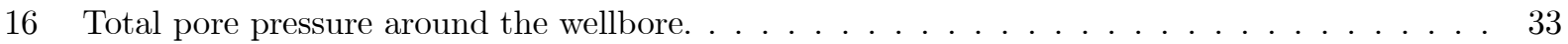

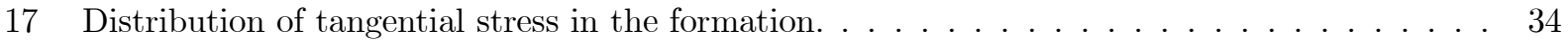

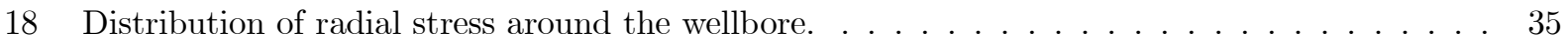

19 Tangential stress in the vicinity of the well, in the $\mathrm{S}_{\max }$ direction. . . . . . . . . . 36

20 Uniformly pressurized, cooled crack. . . . . . . . . . . . . . . . . . 37

21 Magnitude of maximum crack opening as a fucntion of time for mode $1 \ldots \ldots$. . . . . . 38

22 Normalized crack opening for mode $1 . \ldots \ldots$. . . . . . . . . . . . . . 39

23 Magnitude of crack opening due to an applied pore pressure. . . . . . . . . . . . . . 40

24 Normalized crack opening due to pore pressure loading. . . . . . . . . . . . . . . . . 41

25 Maximum crack opening due to cooling, also shown is the steady-state analytical value. . . . 42

26 Time evolution of normalized (with respect to Mode 1 value at $t^{0+}$ ) crack width. . . . . . . . 43

27 Location of the point where DD's are calculated for SIF determination. . . . . . . . . . . . 44

28 Magnitude of SIF for mode 1 loading. . . . . . . . . . . . . . . . . . . 45

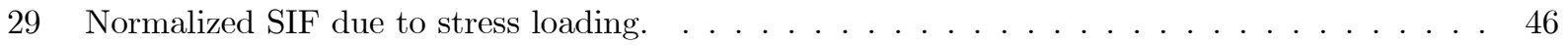

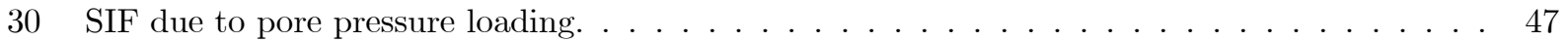

31 Normalized SIF for pore pressure loading. . . . . . . . . . . . . . . . . 48

32 Temporal evolution of stress intensity factor due to cooling. . . . . . . . . . . . . 49

33 Crack path under uniaxial stress. . . . . . . . . . . . . . . . . . . . . 52

34 Crack path under biaxial stress. . . . . . . . . . . . . . . . . . . . 53

35 Crack growth near a circular inclusion under biaxial stress $(R / a=0.5 ; q / p=2) \ldots \ldots$. . . 53

36 Crack growth under biaxial stresses near the circular inclusion $(R / a=0.75 ; q / p=2) \ldots \ldots \quad 54$

37 Crack growth near a circular inclusion in a biaxial stress field $(R / a=1.0 ; q / p=2)$. . . . . 54

38 Problem geometry for studying fracture initiation and propagation from a wellbore. . . . . . 56

39 Dependence of the fracture path on dimensionless parameter $\beta \ldots \ldots \ldots$. . . . . . . . 57

40 Variation of propagation pressure along the crack path ( $n$ is number of the propagation step,

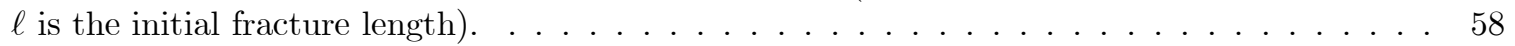

41 Dependence of the minimum of propagation pressure on $\Pi$. . . . . . . . . . . . 58

42 Dependence of the fracture path on the inclination angle $\alpha$ (slow rate) . . . . . . . . . 60

43 Variation of propagation pressure along the paths (slow rate) . . . . . . . . . . 60

44 Dependence of the fracture path on preexisting crack length. . . . . . . . . . . . 61

45 Dependence of the initiation pressure on the initial crack length, $\ell \ldots \ldots \ldots \ldots$

46 Dependence of the fracture path on the inclination angle $\alpha$ (fast rate) . . . . . . . . . . . 62

47 Variation of the propagation pressure along the paths (fast rate) . . . . . . . . . . . 62

48 Problem geometry for fracture propagation from a wellbore in the vicinity of a fault. . . . . 63 
49 The trajectories of isolated cracks under fluid pressure (slow rate of loading).

50 Fractures trajectories for differenet inclinations of the preexisting crack (slow pressurization, $\beta=3.95) \ldots \ldots \ldots \ldots \ldots \ldots \ldots \ldots \ldots \ldots \ldots$

51 Fractures trajectories for different inclinations of the preexisting crack (fast pressurization, $\beta=3.95$ ). . . . . . . . . . . . . . . . . . . . . . . . 67

52 Fractures trajectories in the close vicinity of a gently inclined fault with $\sigma_{H}-\sigma_{h}=2 \mathrm{MPa}$, $\beta=1.19$. . . . . . . . . . . . . . . . . . . . . . . . . 69

53 Fractures trajectories in the close vicinity of a steep fault , $\ell=0.56 \mathrm{~m}, \sigma_{H}-\sigma_{h}=2 \mathrm{MPa}$. . 70

54 The role initial crack length, $\ell=0.06 \mathrm{~m}, \sigma_{H}-\sigma_{h}=2 \mathrm{MPa}$. . . . . . . . . . . . . . . 71

55 Trajectories due to the modification of the stresses, $\sigma_{H}-\sigma_{h}=6 \mathrm{MPa}, \ell=0.56 \mathrm{~m}$ (compare with Figure 53). . . . . . . . . . . . . . . . . . . . . . . . . . . 72

56 Trajectories near a steep fault at a larger distance, with $\sigma_{H}-\sigma_{h}=6 \mathrm{MPa}$ and $\ell=0.06 \mathrm{~m}$ (compare with Figure 54). . . . . . . . . . . . . . . . . . . . . . . . 73

57 Crack paths for the case when the maximum stress is in the x-direction. . . . . . . . . . . .

58 The effect of friction on the fracture path (small friction angle). . . . . . . . . . . . . . .

59 The effect of friction on the fracture path (larger friction angle). . . . . . . . . . . . . . . .

60 Crack propagation from a wellbore near a fault, $\beta=3.95$ (compare with Figure 50). . . . . .

61 Crack emanating from a wellbore near a steep fault, $\beta=3.95 \ldots \ldots . . . . . . . . .$.

62 Crack paths for the case of slow pressurization, with $\sigma_{H}=15 \mathrm{MPa}, \sigma_{H}=10 \mathrm{MPa}, K_{I C}=2$ $\mathrm{MPa} \cdot \mathrm{m}^{\frac{1}{2}}(\beta=0.79) . \ldots \ldots \ldots \ldots \ldots \ldots \ldots \ldots$

63 Crack paths for fast pressurization rate, with $\sigma_{H}=15 \mathrm{MPa}, \sigma_{H}=10, \mathrm{MPa}, K_{I C}=2 \mathrm{MPa} \cdot \mathrm{m}^{\frac{1}{2}}$ $(\beta=0.79)$. . . . . . . . . . . . . . . . . . . . . . . 80

64 Real time propagation of en echelon cracks under isotropic tension. . . . . . . . . . . . . . 85

65 Hydraulic fracture propagation from a wellbore. Also shown, are the graphs of pressure and SIF history. . . . . . . . . . . . . . . . . . . . . . . . 85

66 Fracture propagation in heterogeneous rock. . . . . . . . . . . . . . . . . 86

67 Plot of $T_{w}^{*}\left(t_{D}\right) \ldots \ldots \ldots \ldots \ldots . \ldots \ldots$

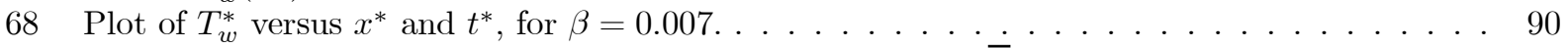

69 Difference of $T_{w}^{*}$ between Model I and Model II for the case $\bar{\beta}=0.1$. . . . . . . . . . . . . . 91

70 Velocity profile in parallel plates. . . . . . . . . . . . . . . . . . . . . . . . 91

71 Difference of $T_{w}^{*}$ between Model II and Model III for the case $\bar{\beta}=0.007$ and $P_{e}=100$. . . . 93

72 Geometry of mathematical model. . . . . . . . . . . . . . . . . . . . . . . . 93

73 Heat balance in a fracture segment. . . . . . . . . . . . . . . . . . . . . . . . . 94

74 Extraction Temperature $T^{*}$ as function of time, at $20 \%$ fluid loss (dash line) and no fluid loss (solid line). . . . . . . . . . . . . . . . . . . . . . . . . . 96

75 Extraction Temperature $T^{*}$ as function of time, at $50 \%$ fluid loss (dash line) and no fluid loss (solid line). . . . . . . . . . . . . . . . . . . . . . . . . . . 97

76 Extraction temperature $T^{*}$ as function of time, with $50 \%$ injection rate without leakoff (dash line), and full injection rate without leakoff (solid line). . . . . . . . . . . . . . . . . 98

77 Heat extraction rate per unit height of fracture (dash line: 50\% leakoff; solid line: no leakoff) 98

78 Temperature distribution in the reservoir and the fracture $(y=0)$ at $t=30$ yr., $50 \%$ leakoff. 99

79 Temperature distribution in the reservoir and the fracture $(y=0)$ at $t=30$ yr., no leakoff. $\quad .99$

80 Normalized temperature distribution in the fracture based on 1-D and 2-D heat conduction models. . . . . . . . . . . . . . . . . . . . . . . . . . . . . 104

81 Normalized extraction temperature based on 1-D and 2-D heat conduction models. . . . . . 105

82 Normalized water temperature extracted form a fracture using the 2D BEM (fluid velocity of $0.5 \mathrm{~cm} / \mathrm{sec}$, see Cheng et al., 2001 for fluid \& rock properties). . . . . . . . . . . . . . . . 106

83 Thermal stresses on the fracture surface in an injection/extraction operation. . . . . . . . 107

84 Computational mesh for a planar fracture. . . . . . . . . . . . . . . . . . . . . 113

85 An infinite fracture with an injection well. . . . . . . . . . . . . . . . . . . 113

86 Normalized temperature distribution in the infinite fracture for various times. . . . . . . . 114 
87 Computational mesh for heat extraction from a circular crack showing flow vectors. . . . . . 114

88 Temperature distribution in circular fracture at $t=3$ days, based on integral equation solution.115

89 Comparison of the 1-D FVM and analytical results. . . . . . . . . . . . . . . 115

90 Extraction temperature for various fracture sizes. . . . . . . . . . . . . . . . . 116

91 A fracture with an arbitrary geometry. . . . . . . . . . . . . . . . . 116

92 Fluid flow in an arbitrarily shaped fracture with one injection well and two extraction wells. . 117

93 Normalized temperature deficit in a fracture with an injection well and two extraction wells. . 117

94 Approximation of the crack path at each propagation stage . . . . . . . . . . . . . 139

95 Part 1 of the fowchart. . . . . . . . . . . . . . . . . . . . . . . 142

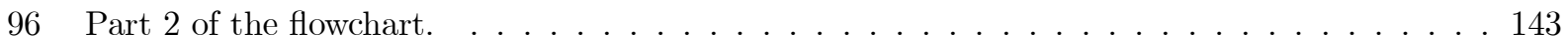

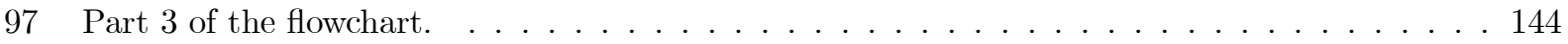

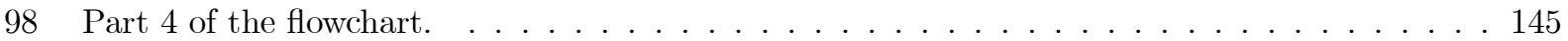

99 Window showing establishing a connection to the server. . . . . . . . . . . . . . 145

100 Window showing program access and run options. . . . . . . . . . . . . . 146

101 Window showing accessing a file. . . . . . . . . . . . . . . . . . . 147

102 Window for choosing output plot color, size, etc. . . . . . . . . . . . . . . 148

103 Window showing an input data file. . . . . . . . . . . . . . . . . . 151

104 Window showing file access procedure . . . . . . . . . . . . . . . . . . 152

105 An example of an output file generated by the program (Result.dat). . . . . . . . . . . 153

106 Window showing the history of files accessed. . . . . . . . . . . . . . 153

107 Window showing a brief description of the program and various engine options. . . . . . . 154

108 Window showing program's response to blank input lines. . . . . . . . . . . . . 155

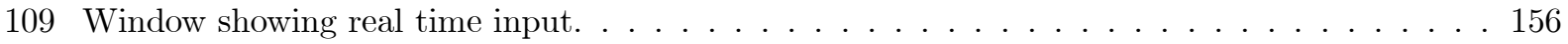

110 Window showing a message in response to a RUN command. . . . . . . . . . . . . 157

111 Window showing program's response to a download request. . . . . . . . . . . . . . 157

112 A plot generated from an output file. . . . . . . . . . . . . . . . 158

113 Window showing example output data files. . . . . . . . . . . . . . . . 159

114 Window showing program's response to a pause command issued by user. . . . . . . . . . 160

115 Window showing options upon pausing the program . . . . . . . . . . . . . 161

116 First window for changing input parameters after pausing the program. . . . . . . . . . . 161

117 Second window for changing problem input parameters. . . . . . . . . . . . . . . . 162

118 Third window for changing input parameters (e.g., shear stress) . . . . . . . . . . . . . . 162

119 Real time illustration of the crack path adjusting to new boundary conditions. . . . . . . 163 


\section{ACKNOWLEDGEMENTS}

The PI (A. Ghassemi) has benefited from the contributions of many individuals in completing this project, including graduate students, post-doctoral fellows, subcontractors, and consultants; whose efforts are reflected in the publications listed at the end of this report. S. Mogilevskaya provided her Cgrowth and Leo codes and conducted a number of fracture propagation studies. The algorithms were later combined by A. Savitski; who also implemented a preliminary program, under the supervision of E. Detournay, to calculate thermal stresses along a crack in a homogeneous medium. V. Koshelev contributed by working on development of a thermoelastic complex variable boundary element method and also investigating the influence of faults and inhomogeneities on fracture propagation. Q. Zhang participated in poro-thermoelastic modeling and program development and its applications to crack and wellbore problems. S. Satyan developed the first version of the client-server architecture and added the interface algorithms to the fracture propagation codes; his work was modified by J. Wang, who also assisted with numerous illustrative examples and improvements. A. Cheng carried out the formulation and solution of the $2 \mathrm{D}$ heat extraction problem using the boundary element method (BEM), and also formulated the 3D version. S. Tarasovs participated in the BEM implementation of the heat extraction problem and the solution of the resulting integral equations as well as the finite volume equations; he also developed the interface for the resulting algorithms. The research also benefited from consultations with A. Simakin, A. Diek, and A. Linkov. 


\section{DISCLAIMER}

This information was prepared as an account of work sponsored by an agency of the U.S. Government. Neither the U.S. Government nor any agency thereof, nor any of their employees, makes any warranty, express or implied, or assumes any legal liability or responsibility for the accuracy, completeness, or usefulness of any information, apparatus, product, or process disclosed, or represents that its use would not infringe privately owned rights. References herein to any specific commercial product, process, or service by trade name, trademark, manufacturer, or otherwise, does not necessarily constitute or imply its endorsement, recommendation, or favoring by the U.S. Government or any agency thereof. The views and opinions of authors expressed herein do not necessarily state or reflect those of the U.S. Government or any agency thereof. 


\section{Introduction}

\subsection{General Aspects of the Study}

Geothermal energy is recovered by circulating water through heat exchange areas within a hot rock mass. Geothermal reservoir rock masses generally consist of igneous and metamorphic rocks that have low matrix permeability. Therefore, cracks and fractures play a significant role in extraction of geothermal energy by providing the major pathways for fluid flow and heat exchange. Thus, knowledge of conditions leading to formation of fractures and fracture networks is of paramount importance. Furthermore, in the absence of natural fractures or adequate connectivity, artificial fracture are created in the reservoir using hydraulic fracturing. At times, the practice aims to create a number of parallel fractures connecting a pair of wells. Multiple fractures are preferred because of the large size necessary when using only a single fracture. Although the basic idea is rather simple, hydraulic fracturing is a complex process involving interactions of high pressure fluid injections with a stressed hot rock mass, mechanical interaction of induced fractures with existing natural fractures, and the spatial and temporal variations of in-situ stress. As a result it is necessary to develop tools that can be used to study these interactions as an integral part of a comprehensive approach to geothermal reservoir development, particularly enhanced geothermal systems. In response to this need we have set out to develop advanced thermo-mechanical models for design of artificial fractures and rock fracture research in geothermal reservoirs. These models consider the significant hydraulic and thermo-mechanical processes and their interaction with the in-situ stress state. Wellbore failure and fracture initiation is studied using a model that fully couples poro-mechanical and thermo-mechanical effects. The fracture propagation model is based on a complex variable and regular displacement discontinuity formulations. In the complex variable approach the displacement discontinuities are defined from the numerical solution of a complex hypersingular integral equation written for a given fracture configuration and loading. The fracture propagation studies include modeling interaction of induced fractures with existing discontinuities such as faults and joints. In addition to the fracture propagation studies, two- and three-dimensional heat extraction solution algorithms have been developed and used to estimate heat extraction and the variations of the reservoir stress with cooling. The numerical models have been developed in a user-friendly environment to create a tool for improving fracture design and investigating single or multiple fracture propagation in rock.

\section{$1.2 \quad$ Issues \& Scope}

Fracture initiation and propagation are very important in development of geothermal reservoirs particularly EGS. Other important coupled processes that control flow and heat extraction in a geothermal reservoir include ( $i$ ) fracture closure/opening in response to changing effective normal stress, (ii) fracture shear dilation during stimulation and circulation, (iii) thermoelastic effects in stimulation and circulation operations, and (iv) chemical dissolution and precipitation during circulation.

The objective of this project is to develop an advanced two-dimensional, thermo-mechanical model that allow investigation of these processes (with the exception of chemical phenomena) in a geothermal environment. This has been accomplished by developing rock mechanics models that consider significant hydraulic and thermo-mechanical processes and their interaction with the in-situ stress state. However, the number

and complexity of the processes involved in drilling, stimulation, and circulation preclude development of a single model for treatment and analyses of various problems. Thus, a number of analytic and numerical 
models have been developed to investigate various aspects of a problem of interest. Specifically, the research activities consist of:

- development of a poro-thermoelastic model for wellbore failure analysis and fracture initiation

- development of a two-dimensional boundary element code for modeling single/multiple fractures and fracture propagation near a natural discontinuity such as joints and faults; and studying thermoelastic effects

- analyses of variations in fracture propagation as a function of changes in the reservoir stress state;

- theoretical study of fluid flow and heat exchange in artificial and natural fractures;

- integration of the analytic and numerical algorithms in a user friendly environment.

By considering the relevant rock mechanics issues and processes in fracture design, the proposed project represents an advancement of the state-of-the-knowledge and technology in high pressure thermo-mechanical rock/fluid interactions; and provides the domestic geothermal industry with sophisticated cutting-edge technology. It is anticipated that utilizing this tool will improve design of artificial fractures and enhanced geothermal systems.

\section{Hydraulic Fracturing in Geothermal Reservoirs: Fundamental Concepts \& Mathematical Framework}

Hydraulic fracturing and stimulation of geothermal reservoirs involves a number of processes; namely

- fracture initiation

- fracture propagation

- fluid flow in a deformable fracture

- fluid diffusion into the rock

- heat transfer between the fluid and rock

Each of these aspects forms, in itself, a complex problem which has been the subject of many investigations and merits further study. A detailed examination of these processes with the intent of simultaneously improving their treatment is beyond the objectives of the present investigation. However, it is beneficial to review each area to gain a better understanding of the problems and how they are treated in the present work. We begin with fracture initiation and propagation.

During pressurization of the borehole, the solid (rock) is subjected to a system of external loads under certain environmental conditions (stress, temperature, chemical state). The response of the rock to imposed loads is simply one of deformation in the sense that the rock remains topologically unchanged [10]. Application of the load will eventually cause the formation of a fracture creating new surface areas within the rock mass. Therefore, from a mathematical viewpoint, two types of problems may be distinguished: fracture initiation and fracture propagation. 


\subsection{Fracture Initiation}

In dealing with the fracture initiation problem, one needs to evaluate the critical level of the applied loads that correspond to inception of a fracture. An appropriate fracture criterion is necessary to augment the analysis of deformation and stresses, for the purpose of relating it to the fracture resistance of the rock. Several different failure criteria are in existence for use in various applications [25]:

- Maximum tensile stress theory; according to this theory, failure initiates when the minimum principal stress component, $\sigma_{3}$, reaches the tensile strength, $T_{0}$, of the material, $\sigma_{3}=-T_{0}$.

- Coulomb's theory; it is the maximum shear stress criterion which postulates that fracture will occur at a point in the material for a specific value $S_{0}$, referred to as the shear strength $\frac{\left(\sigma_{1}-\sigma_{3}\right)}{2} \geq S_{0}$ with $\sigma_{1}$ and $\sigma_{3}$ representing the major and minor principal stresses, respectively. According to this theory the failure plane will bisect the angle between the minor and major principal stresses so that in a triaxial test, the plane of failure should be at $45^{\circ}$ to the axial stress. This conclusion, however, is not observed experimentally. The orientation of the failure plane varies with rock type and is less than $45^{\circ}$ to the direction of maximum compression.

- Coulomb-Navier (Modified max. shear criterion); Coulomb's theory was modified by Navier to include the influence of the normal stress acting on the plane of failure which tends to increase the shear resistance of the material [28]. Representing the normal stress by $\sigma$ and the shear stress by $\tau$, the theory stipulates that failure occurs when the magnitude of the shear stress acting on the failure plane reaches a value given by $|\tau|=S_{0}+\mu^{*} \sigma$ in which $\mu^{*}$ is the coefficient of internal friction. This criterion for failure indicates that the angle between the failure plane and the direction of maximum load, $\theta$, will be less than $45^{\circ}$ which is in agreement with experimental evidence [28].

- Mohr's Theory; it postulates that a material fractures or begins to deform permanently when the shear stress acting on the plane of failure increases to a value which is a function of the normal stress acting on the same plane $\tau=f(\sigma)$. However, if the largest tensile principal stress has reached the tensile strength of the material, the maximum tensile stress theory, mentioned previously, is assumed. The form of $f(\sigma)$ is determined experimentally. Unlike the previously described criteria, Mohr's theory of failure predicts the direction of failure in addition to the state of stress at which failure occurs. Mohr's theory also implies that the value of the intermediate stress does not affect the shear stress, $\tau$, so that failure is independent of this stress. It also indicates that failure will not occur in hydrostatic compression consistent with experimental observation. It should be mentioned that the Coulomb-Navier and Mohr criteria are identical if the coefficient of internal friction is constant [2].

- Griffith's Theory; in contrast to the previously described empirical criteria that do not relate failure to any internal mechanisms or processes taking place in the rock material, the [14] theory is based on a given mechanism and relates failure to it. According to Griffith, two conditions are necessary for fracture; a stress condition and an energy condition, i.e., the cohesive forces must be exceeded and the energy required to form two surfaces in the solid must be provided. Together, these conditions are sufficient to ensure fracture. The first condition provides the basis for a failure criterion, while many fracture propagation criteria originate from the energy condition. Griffith's theory is based on the assumption that rocks contain flaws such as microcracks. When the rock body is subjected to 
external loads, stress concentrations develop around these features. These stress concentrations cause crack initiation and growth, which will ultimately cause macroscopic failure. Griffith considered the problem of a thin, linearly elastic, isotropic, infinite plate, containing a thin elliptical slit of length $2 a$ subjected to a tensile stress $\sigma$. The normal stress developed at the tip of the crack, due to the applied load, is given by [21]:

$$
\sigma_{t}=\sigma\left(1+\frac{4 a}{2 b}\right)
$$

in which $2 a$ and $2 b$ represent the major and minor axes of the elliptical hole. Thus for sharp slits (i.e. large values of $\frac{a}{b}$ ) the stress, $\sigma_{t}$, could be large enough to cause failure at moderate levels of applied load. This formed the basis for Griffith's analysis and formulation of his stress condition for failure. [15] derived his failure criterion by considering the variation of the tangential stress $\sigma_{t}$ at the surface a flat elliptical crack under the action of two-dimensional stresses $\sigma_{1}, \sigma_{2}$ at infinity and obtained:

$$
\frac{\left(\sigma_{1}-\sigma_{3}\right)^{2}}{\sigma_{1}+\sigma_{3}}=8 T_{0}
$$

If $\sigma_{3}=0$ and $\sigma_{1}=C_{0}$, the uniaxial compressive strength of the rock, the above relation indicates that $T_{0}=\frac{C_{0}}{8}$, i.e., tensile strength is exactly one eighth of the uniaxial compressive strength, a condition that is not consistent with experimental observations. The compressive strength of most rocks varies from 10 to 100 times the tensile strength [16]. In addition, [17] showed that the failure criterion corresponds to a Mohr's envelope at failure is given by:

$$
\tau^{2}+4 T_{0} \sigma=4 T_{0}^{2}
$$

This is a parabolic criterion, and so it is not valid for many rocks (especially igneous). Typically brittle rocks such as granite and quartzite, for which the Griffith criterion is most likely valid, have a straight envelope in compression, in disagreement with the normal Griffith criterion [3]. When modified to include closing of cracks in compression [27], the prediction turns out to be linear and identical to the Navier-Coulomb criterion, upon complete closure of cracks. This modified version gives an improved ratio of the compressive-to-tensile strengths for rocks of 12 , but the ratio is still low in comparison with that obtained from measurements [3]. It should also be kept in mind that the Griffith theory does not predict the path of a growing crack except when loaded normal to its surface (simple tension). It does, however, indicate that, in compression, the direction in which fracture is initiated is not in the direction of the original crack and turns towards the direction of the maximum principal stress. However, Griffith's failure criterion is valid in both tension and compression. This is very important and adds to the attractiveness of the theory, particularly when viewed in light of the fact that tension and shear are the two basic modes causing fracture, and there is no fundamental compression mechanism of failure. Finally, similar to Coulomb and Mohr criteria; Griffith's theory predicts that the intermediate principal stress has no effect on the strength.

\subsubsection{Hydraulic fracture initiation}

The maximum tensile stress theory is used herein to predict the tensile failure of the borehole wall $\left(\sigma_{3}=-T_{0}\right)$ and hydraulic fracture initiation. For a borehole in an impermeable rock formation, whose axis is parallel to 
the major principal stress, the condition for failure and creation of a tensile fracture is given by:

$$
3 \sigma_{h, \min }-\sigma_{H, \max }-p+T=p_{b}
$$

in which $p$ is the initial pore pressure, and $p_{b}$ is the breakdown pressure which is viewed as the pressure at which the crack is formed; often assumed to correspond to the initial peak of the pressure-time record. $T$ represents the tensile strength of the intact rock material, and $p$ is the reservoir pore pressure. The term $\left(3 \sigma_{h, \min }-\sigma_{H, \max }\right)$ in the above equation represents the least stress concentration along the borehole wall due to the far-field horizontal stresses, and is obtained from the Kirsch solution [12].

However, there are indications that shear may be a mode of failure in initiation of hydraulic fractures from inclined wellbores [13], [29], [41]. Indeed, some investigators [4], [30] argue that a hydraulic fracture is induced by shear rather than tensile failure and use a Mohr-Coulomb shear failure criterion.

As noted by [5], neither the tensile nor the shear failure criterion is capable of predicting the high breakdown pressures observed in the laboratory as well as its dependence on borehole size and injection rate. A fracture mechanics model [33],[56]-[59] based on the concept of unstable fracture propagation using the fracture toughness criterion, $K_{I}=K_{c}$, seems more promising. Introduction of an additional condition,

namely, $\frac{\partial\left(K_{I}-K_{c}\right)}{\partial L} \geq 0$ makes it possible to take into account phenomena such as pumping rate- and sizedependence of the breakdown pressure.

Fracture initiation from a wellbore may be treated using the classical strength of materials approach [1], [54] with Eq. 4 or the fractures mechanics approach [57]-[59]. The fracture mechanics approach can be considered by using a fracture propagation model based on the principles of fracture mechanics. In some instances it provides a better estimate of the breakdown pressure. However, because of its practicality, the classical approach is the one that is widely used and is adopted here.

The above Eq. 4 for fracture initiation pressure does not take into account the influence of poroelastic and thermoelastic effects. Using the classical approach, fracturing of a vertical well in a cooled rock was considered in [60]; it was found that thermal stresses can impose a significant influence on hydraulic fracturing and interpretation of its results to determine the maximum far-field stress. Cooling the rock induces tensile stresses and results in a lower wellbore pressure for fracture initiation. Both analytical and numerical models have been developed that can calculate the stress distribution around a wellbore of arbitrary orientation while considering, thermoelastic, and poroelastic effects. These are useful for calculating the failure pressure and its location around the wellbore based on the classical approach. The details of the governing equation and solution methodologies can be found in [61] for the analytical approach; and in [125] for the numerical solution. Certain aspects of the latter appear in this report also.

Once the onset of failure and fracture initiation is established from the knowledge of the stresses and the limit of the material's ability to carry an applied load (i.e., a failure criterion), the question arises as to if and how the fracture propagates. This is the subject of fracture propagation modeling portion of this project. However, the numerical model can also be used to study fracture initiation using a fracture mechanics approach.

\subsection{Fracture Propagation}

Whether a fracture subject to a system of loads propagates, and if the propagation is stable or not is the subject of the field of fracture mechanics. In this work fracture propagation is studied within the framework 
of what is known as the field of Linear Elastic Fracture Mechanics (LEFM). The mathematical framework of LEFM is described in detail below.

According to LEFM, near the crack tip the displacements are proportional to $\sqrt{r}$ and the stresses contain a singularity of the form $\frac{1}{\sqrt{r}}$. This modern theory of fracture mechanics has its roots in the work of [14] who emphasized the energy dissipated in an increment of fracture extension, which is now referred to as the energy release rate, $G$.

In the previous section, Griffith's theory was described within the framework of a failure criterion. It was indicated that to initiate a fracture it is necessary to have a stress concentration which is provided by inherent microcracks or flaws. This stress condition is necessary but not sufficient for fracture propagation as very sharp flaws may be present within a loaded body without causing failure. Indeed, Inglis' results show that the stresses at the edge of a sharp crack can tend to infinity. This paradox can be understood by the second aspect of Griffith's theory, namely the energy balance which leads to a critical condition for fracture propagation. It is written as an equality between the change in the potential energy due to an increment of crack extension and the energy required to create two new surfaces [26]:

$$
\frac{d(W-U)}{d A}=\gamma
$$

where $W$ and $U$ are the external work done on the body and its internal strain energy, respectively, and $A=4 B a$ is the crack surface area for an internal fracture of length $2 a$ in a body of thickness B (it equals unity under plain strain condition). Westergaard's solution [46] for the opening displacement, $v$, of the Griffith crack is given by:

$$
v=\frac{2 \sigma}{E^{\prime}}\left(a^{2}-x^{2}\right)^{\frac{1}{2}}
$$

in which $E^{\prime}=E$ for plane stress, $E^{\prime}=\frac{E}{1-\nu^{2}}$ for plane strain, and $\sigma$ is the applied stress. Thus, it can be shown that:

$$
W-U=4 B \int_{0}^{a} \frac{1}{2} \sigma v(x) d x=\frac{B \pi a^{2} \sigma^{2}}{E^{\prime}}
$$

Assuming fixed grip conditions (constant displacement at the boundary) so that the external work equals zero, and substituting Eq. (5) gives the strain energy released when the crack grows by an amount $d a$ :

$$
\frac{d U}{d a}=\frac{\pi \sigma^{2} a}{2 E^{\prime}}
$$

This can be solved to yield the magnitude of the stress at failure:

$$
\sigma=\left(\frac{2 E^{\prime} \gamma}{\pi a}\right)^{\frac{1}{2}}
$$

The strain energy release rate at one tip is:

$$
G=\frac{\pi \sigma^{2} a}{4 E^{\prime}}
$$

Therefore, Griffith's energy balance states that crack growth occurs when $G$ reaches a critical value, $G_{c}$; a material property representing its resistance to crack growth. The parameter $G$ can be expressed in terms of tip parameters. This became possible as a result of the work of Westergaard [46] who used stress functions 

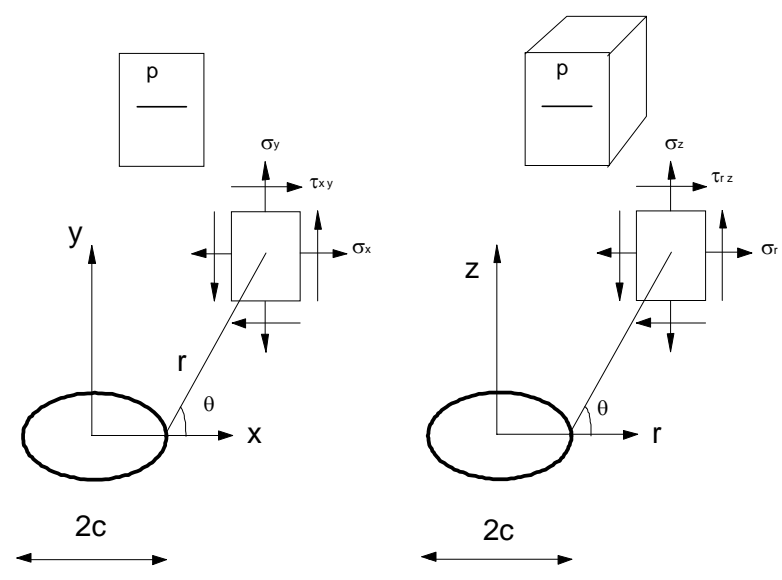

Figure 1: State of stress near the tip of a crack.

derived from complex potentials to find expressions for distribution of stresses around the tip of cracks with thicknesses approaching zero. Sneddon [40] applied Westergaard's results to both two- and three-dimensional pressurized cracks. The stress field in the vicinity of the tip of a two-dimensional crack is given by:

$$
\left[\begin{array}{c}
\sigma_{x} \\
\sigma_{y} \\
\tau_{x y}
\end{array}\right]=P \sqrt{\frac{a}{2 r}}\left[\begin{array}{l}
\frac{3}{4} \cos \frac{\theta}{2}+\frac{1}{4} \cos \frac{5 \theta}{2} \\
\frac{3}{4} \cos \frac{\theta}{2}-\frac{1}{4} \cos \frac{5 \theta}{2} \\
\frac{3}{2} \sin \theta \cos \frac{3 \theta}{2}
\end{array}\right]
$$

and for a penny-shaped crack,

$$
\left[\begin{array}{c}
\sigma_{r} \\
\sigma_{z} \\
\tau_{z r}
\end{array}\right]=\frac{2 P}{\pi} \sqrt{\frac{a}{2 r}}\left[\begin{array}{l}
\frac{3}{4} \cos \frac{\theta}{2}+\frac{1}{4} \cos \frac{5 \theta}{2} \\
\frac{3}{4} \cos \frac{\theta}{2}-\frac{1}{4} \cos \frac{5 \theta}{2} \\
\frac{3}{4} \sin \theta \cos \frac{3 \theta}{2}
\end{array}\right]
$$

where $P$ is the internal pressure on the crack face, $a$ is the fracture half-length in two-dimensions and its radius in three dimensions, $r$ is the distance from a point to the crack tip, and $\theta$ represents the angle that $r$ makes on the central plane of the crack (Figure 1).

As can be observed, and noted by [40], the above expressions for stress components around the tip in three dimensions differ from those in the two-dimensional case by a numerical factor only.

Writing the second of Equations (11) for the case $\theta=0$, and translating the center of the coordinate system to the center of the crack yields:

$$
\sigma_{y}=\frac{K}{\sqrt{2 \pi(x-a)}}
$$

where $K$ is the stress intensity factor. It is a quantity which depends on the geometry and loading and has the value $\sigma \sqrt{2 \pi a}$ where $\sigma=\lim _{x \rightarrow a} \sigma_{y}$. The crack opening displacement in terms of $K$ is given by [26]:

$$
v=\frac{(1+\nu)(\kappa+1)}{E} K\left(\frac{a-x}{2 \pi}\right)^{\frac{1}{2}}
$$




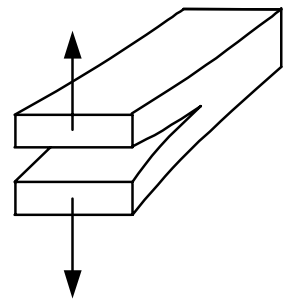

I

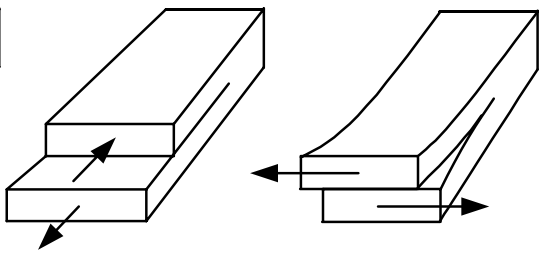

II
III

Figure 2: A sketch of three fundamental modes of fracture.

in which $\kappa$ equals $\left(\frac{3-\nu}{1+\nu}\right)$ for plane stress and $(3-4 \nu)$ for plane strain, and $\nu$ represents the Poisson's ratio. Using these equations, Irwin [22] calculated the work required to close the crack that has extended by an amount $\Delta a$, which may be equated to the product of the energy release rate and the increment of crack extension. Therefore,

$$
G \Delta a=2 \int_{a}^{a+\Delta a} \frac{1}{2} \sigma_{y}(x) v(x-\Delta a) d x
$$

so that:

$$
G=\frac{K^{2}}{E^{\prime}}
$$

This expression, derived by Irwin [22], relating the Griffith's strain energy release rate, a global parameter, to the stress intensity factor, a local crack-tip quantity, is considered as a major contribution. It shows that Griffith's criterion for crack growth is essentially identical with that of crack growth when the stress intensity factor, $K$, reaches a critical value $K_{c}$. This parameter is known as the fracture toughness. It is a material property which can be determined experimentally, and is known to be a function of temperature, confining pressure, and moisture content [23]. But, when defining the fracture toughness, $K_{c}$, it is assumed that the material is homogeneous and linearly elastic. These conditions do not generally hold true in the case of rock, and nonlinear behavior prevails at the crack tip in case of a homogeneous, linear elastic rock. The size of this zone has a large influence on the value of the fracture toughness and results in a scale effect. Indeed, laboratory tests indicate that fracture toughness increases with an increase in crack length or specimen size [24]. For large crack lengths or specimens, $K_{c}$ becomes independent of size, however, and approaches an asymptotic value considered to be an intrinsic material parameter called fracture toughness.

When applied to a hydraulic fracture, the above criterion predicts that the higher the fracture toughness, the higher the pressure required to extend the fracture, and the longer the fracture, the lower the pressure required for its extension (if the overburden remains constant).

Hydraulically-driven fractures are considered to propagate in Mode I or the opening mode, however, mixmode propagation is possible but is not considered in the modeling efforts. However, it is useful to review other modes of fracture propagation as shear growth may take place on a macro-level in some geologic situations, out-of-plane propagation, and injection operations from pre-existing joints.

In addition to a crack loaded in uniaxial tension, Irwin also considered other configurations of fracture loading and classified them into three independent modes. Mode-I is the above considered problem, Mode-II 
refers to a crack loaded in shear, i.e., the load is tangential to the crack surface in the direction of propagation; and Mode-III is defined by a crack loaded by a tangential load in the direction perpendicular to the direction of propagation.

This is also called the antiplane mode (Figure 2). The equations for stresses and displacement for these problems are as given below:

\subsubsection{Mode-I or opening mode}

In this mode, the displacements of the crack surfaces are perpendicular to the plane of the crack. Mode-I crack tip stress and displacement fields are [22]:

$$
\begin{gathered}
{\left[\begin{array}{l}
\sigma_{r} \\
\sigma_{\theta} \\
\tau_{r \theta}
\end{array}\right]=\frac{K_{I}}{\sqrt{2 \pi r}} \cos \frac{\theta}{2}\left[\begin{array}{l}
1+\sin ^{2} \frac{\theta}{2} \\
\cos ^{2} \frac{\theta}{2} \\
\sin \frac{\theta}{2} \cos \frac{\theta}{2}
\end{array}\right]} \\
\sigma_{z}=\nu\left(\sigma_{r}+\sigma_{\theta}\right) \tau_{r z}=\tau_{r \theta}=0, \text { for plane strain } \\
\sigma_{z}=\tau_{r z}=\tau_{\theta z}=0, \text { for plane stress } \\
{\left[\begin{array}{l}
u \\
v \\
w
\end{array}\right]=\frac{K_{I}}{4 G} \sqrt{\frac{r}{2 \pi}}\left[\begin{array}{l}
(2 \kappa-1) \cos \frac{\theta}{2}-\cos \frac{3 \theta}{2} \\
-(2 \kappa+1) \sin \frac{\theta}{2}+\sin \frac{3 \theta}{2} \\
0
\end{array}\right]}
\end{gathered}
$$

\subsubsection{Mode-II or sliding mode}

During Mode-II loading, the displacement of the crack surface is in the plane of the crack and perpendicular to the leading edge of the crack. This is caused by in-plane shear. Mode-II crack tip stress and displacement fields are given by [22]:

$$
\begin{aligned}
& {\left[\begin{array}{c}
\sigma_{r} \\
\sigma_{\theta} \\
\tau_{r \theta}
\end{array}\right]=\frac{K_{I I}}{\sqrt{2 \pi r}}\left[\begin{array}{l}
\sin \frac{\theta}{2}\left(1-3 \sin ^{2} \frac{\theta}{2}\right) \\
-3 \sin \frac{\theta}{2} \cos ^{2} \frac{\theta}{2} \\
\cos \frac{\theta}{2}\left(1-3 \sin ^{2} \frac{\theta}{2}\right)
\end{array}\right]} \\
& \sigma_{z}=\nu\left(\sigma_{r}+\sigma_{\theta}\right) \tau_{r z}=\tau_{r \theta}=0, \text { for plane strain; } \\
& \sigma_{z}=\tau_{r z}=\sigma_{\theta z}=0, \text { for plane stress } \\
& {\left[\begin{array}{c}
u \\
v \\
w
\end{array}\right]=\frac{K_{I I}}{4 G} \sqrt{\frac{r}{2 \pi}}\left[\begin{array}{l}
-(2 \kappa-1) \sin \frac{\theta}{2}+3 \sin \frac{3 \theta}{2} \\
-(2 \kappa-1) \cos \frac{\theta}{2} \\
0
\end{array}\right]}
\end{aligned}
$$

In Mode-II the material failure occurs when $K_{I I}$ reaches its critical value $K_{I I c}$, known as the Mode-II fracture toughness.

\subsubsection{Mode-III or tearing mode}

In this mode, the crack surface displacements are in the plane of the crack and parallel to its leading edge. This is caused by out-of-plane shear. Mode-III near tip stress and displacement fields are given as [22]:

$$
\begin{gathered}
{\left[\begin{array}{l}
\tau_{r z} \\
\tau_{\theta z}
\end{array}\right]=\frac{K_{I I I}}{\sqrt{2 \pi r}}\left[\begin{array}{l}
\sin \frac{\theta}{2} \\
\cos \frac{\theta}{2}
\end{array}\right]} \\
\sigma_{z}=\sigma_{r}=\sigma_{\theta}=\tau_{r \theta}=0
\end{gathered}
$$




$$
\begin{gathered}
w=\frac{1}{G} \int \sigma_{x z} d x=\frac{2 K_{I I I}}{G} \sqrt{\frac{r}{2 \pi}} \sin \frac{\theta}{2} \\
u=v=0
\end{gathered}
$$

The elastic stress and displacement fields in mixed mode problems may be obtained from the above results using the superposition principle. For Mode-I and Mode-II combination, for example, this yields:

$$
\left[\begin{array}{l}
\sigma_{r} \\
\sigma_{\theta} \\
\tau_{r \theta}
\end{array}\right]=\frac{K_{I}}{\sqrt{2 \pi r}}\left[\begin{array}{l}
\cos \frac{\theta}{2}\left(1+\sin ^{2} \frac{\theta}{2}\right) \\
\cos ^{3} \frac{\theta}{2} \\
\sin \frac{\theta}{2} \cos ^{2} \frac{\theta}{2}
\end{array}\right]+\frac{K_{I I}}{\sqrt{2 \pi r}}\left[\begin{array}{l}
\sin \frac{\theta}{2}\left(1-3 \sin ^{2} \frac{\theta}{2}\right) \\
-3 \sin \frac{\theta}{2} \cos ^{2} \frac{\theta}{2} \\
\cos \frac{\theta}{2}\left(1-3 \sin ^{2} \frac{\theta}{2}\right)
\end{array}\right]
$$

The corresponding displacement components can be written as:

$$
\begin{aligned}
{\left[\begin{array}{l}
u \\
v
\end{array}\right]=} & \frac{K_{I}}{4 G} \sqrt{\frac{r}{2 \pi}}\left[\begin{array}{l}
(2 \kappa-1) \cos \frac{\theta}{2}-\cos \frac{3 \theta}{2} \\
-(2 \kappa+1) \sin \frac{\theta}{2}+\sin \frac{3 \theta}{2}
\end{array}\right]+ \\
& \frac{K_{I I}}{4 G} \sqrt{\frac{r}{2 \pi}}\left[\begin{array}{l}
-(2 \kappa-1) \sin \frac{\theta}{2}+3 \sin \frac{3 \theta}{2} \\
-(2 \kappa-1) \cos \frac{\theta}{2}
\end{array}\right]
\end{aligned}
$$

In the preceding expressions, $G$ represents the shear modulus. The above stresses and displacements can be expressed in the integral form as:

$$
\begin{gathered}
\sigma_{i j}=\frac{K_{I}}{\sqrt{2 \pi r}} f_{I i j}(\theta)+\frac{K_{I I}}{\sqrt{2 \pi r}} f_{I I i j}(\theta) \\
u_{i}=\frac{K_{I}}{4 g} \sqrt{\frac{r}{2 \pi}} g_{I i}(\theta)+\frac{K_{I I}}{4 g} \sqrt{\frac{r}{2 \pi}} g_{I I i}(\theta)
\end{gathered}
$$

where the stress intensity factors are defined as:

$$
\begin{gathered}
K_{I}=\sigma_{n} \sqrt{\pi a}=\sigma \sqrt{\pi a} \sin ^{2} \beta \\
K_{I I}=\sigma_{n} \sqrt{\pi a}=\sigma \sqrt{\pi a} \sin \beta \cos \beta
\end{gathered}
$$

The above equations show that $K_{I}$ and $K_{I I}$ are function of the crack inclination angle, $\beta$ (see Figure 3 ). Thus, in mixed-mode, the stress intensity factors are functions of the applied load, crack dimensions, and crack inclination with respect to vertical.

These equations form the mathematical framework for fracture propagation studies using the Linear Elastic Fracture Mechanics (LEFM). It can be seen that displacements are proportional to $\sqrt{r}$ and that whatever the mode of loading, the stresses contain a singularity of the form $\frac{1}{\sqrt{r}}$. Therefore, the stress field becomes infinite at the tip for all values of $K$ not equal to zero, for any arbitrary loading. It is apparent that infinite stresses are physically unacceptable and are a direct result of the simplified model used to describe the real physical state. The model assumes perfectly sharp cracks and linear brittle material behavior. But the development of plastic zones ahead of cracks in metals, and microcracking in rocks are manifestations of non-linear behavior and reduce the tip stresses to a finite value. This region of microcracking ahead of a fracture in rocks is often referred to as the process zone and differs from the region of yielding near the crack tip in metals. For rocks, the size and shape of this zone remain the same whether the crack tip location is a free surface or deep within the material, i.e., for plane stress and plane strain. This does not hold for metals [35]. It is for this reason that laboratory observations indicate that thickness has no effect on fracture 

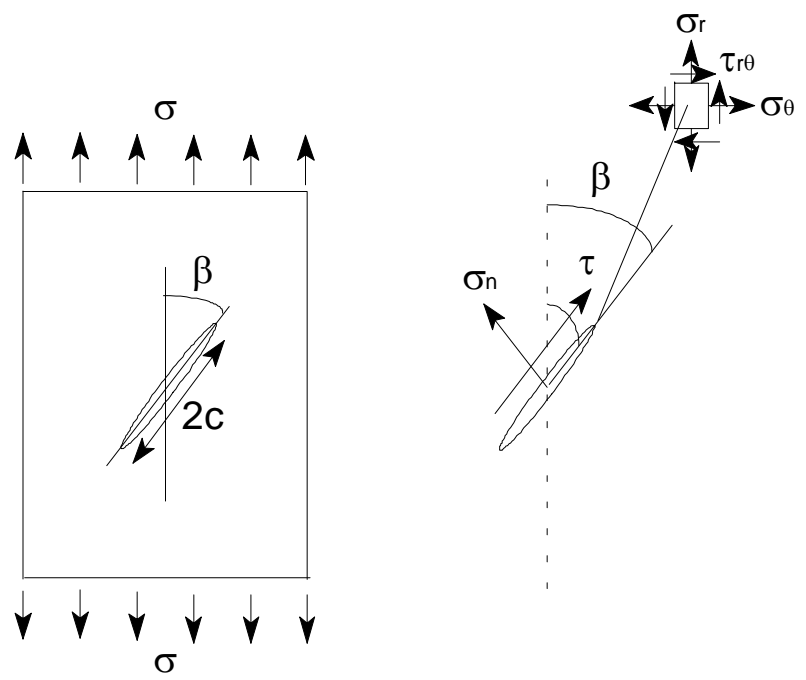

Figure 3: Problem geometry for an inclined crack.

toughness of rocks. So long as the size of the process zone remains small relative to other dimensions of the problem, linear elastic conditions prevail, and LEFM remains applicable. The LEFM is used in the current study.

To resolve the problem of stress singularity at the tip, Barenblatt [52] proposed a new theory in which two postulates were invoked when characterizing the crack tip: (1) that there are intense cohesive forces that act over a small region at the ends of the crack, and (2) that the local distribution of these cohesive forces is the same for a given material under specified conditions. The stress singularity arising from these cohesive forces is such that it cancels out the stress singularity resulting from the applied stresses. This, according to Goodier [11] can be regarded as a third postulate of Barenblatt's theory of equilibrium cracks. The consequence of the third postulate is that the crack closes smoothly and the crack opening displacement has a slope of zero at the tip (Figure 4).

This is in contrast to the opening of a Griffith crack used in this work which is elliptical with a finite radius of curvature and closes with an infinite slope at the tip. The theory of Barenblatt suggest that the cohesive forces increase in response to an increase, from zero, of the applied load on the crack. When these forces are unable to keep the crack from opening, the crack becomes a mobile equilibrium crack and propagation ensues. Barenblatt introduced a new material property called the modulus of cohesion defined as:

$$
K_{0}=\int_{0}^{d} \frac{g(\xi)}{\xi^{\frac{1}{2}}} d \xi
$$

in which $g(\xi)$ is a function describing the intensity of the cohesive forces over a small interval, $d$, at the tip of the crack. The form of this function is unknown so that the above integral cannot be performed. However, Barenblatt showed that it can be expressed in terms of elastic modulus and the surface energy, $\gamma$, as:

$$
K_{0}=(\pi \gamma E)^{\frac{1}{2}}
$$




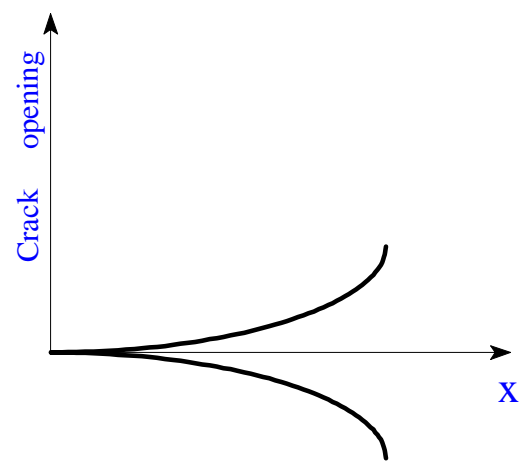

Figure 4: Crack-tip closure resulting from Barenblatt's 3rd postulate.

Using the concept of the stress intensity factor, $K$, Barenblatt's fracture criterion can be written as:

$$
K=\frac{K_{0}}{\pi}
$$

which can be compared to the Griffith's stress condition by using $K=\sigma \sqrt{2 \pi a}$ to obtain the stress required for fracture:

$$
\sigma_{t}=\sqrt{\frac{1}{2 \pi}} \sqrt{\frac{2 E \gamma}{\pi a}}
$$

This differs from the Griffith criterion, Eq. (9), by only a constant.

The Griffith-Irwin criterion is a useful one when applied to situations where the fracture propagates in its own plane. However, the direction of crack propagation is not known $\grave{a}$ priori under combined loading situations so that the above criterion is inadequate and other propagation theories are needed. There are a number of such theories that can be divided into two groups, namely local criteria and global criteria. The maximum circumferential tensile stress, $\left(\sigma_{\theta}\right)_{\max }$, criterion [9] and the minimum strain energy density, $S(\theta)_{\min }$, [39] are examples of local propagation criteria. Propagation is determined by the value of these parameters at a distance from the crack tip. Global criteria are based on the maximum energy release rate, $G(\theta)_{\max }$, associated with crack propagation [20],[34],[44]. In both groups, propagation is assumed to occur at a point where the value of the parameter of interest reaches a critical value characteristic of the material. The propagation direction is determined by the direction in which this parameter has its minimum or maximum as required by the criterion.

In the $\left(\sigma_{\theta}\right)_{\max }$-theory, proposed by Erdogan and Sih [9], the crack extension starts at the tip, in a radial direction, and in a plane perpendicular to the direction of maximum tension. Fracture propagates when $\left(\sigma_{\theta}\right)_{\max }$ reaches its critical value.

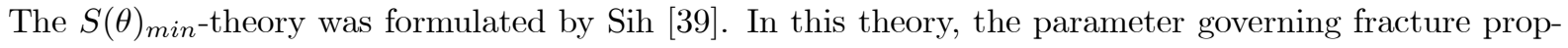
agation is the strain density at the point of propagation. The initial crack growth occurs in the direction along which the strain energy density factor possesses a stationary (minimum) value. The crack extends when the strain energy density reaches a critical value, $S_{\theta}$, a material property. The value of $S_{c}$ is evaluated along a contour $r=r_{0}$, with $r_{0}$ being a property of the material. 
The global energy approach has its roots in the Griffith criterion and is based on the universal principle of energy minimization. It predicts that a fracture will grow in the direction along which the elastic energy release per unit crack extension will be maximum and the crack will start to grow when this energy release rate reaches a critical value. However, according to [36], the application of the energy release rate concept to cracks under mixed mode loading are of doubtful value due to the mathematical difficulties caused by prevailing stress singularities at the crack tip and corner where it is kinked, and because of the problems with accuracy of numerical solutions for solving problems involving stress singularities. Despite the above arguments and that there is no clear reason why the propagation path has to follow the direction of maximum $G(\theta),[20],[34],[31],[32]$ use the above approach to arrive at propagation theories for mixed mode problems involving metals.

From a numerical modeling point of view, the computational effort required to predict the propagation path is very important. The energy release rate needs to be calculated numerically by taking the derivative of the energy release, G, with respect to the fracture length or area in the direction of propagation. The direction of propagation is not known $\grave{a}$ priori so that a trial-and-error method is needed for its determination. For three-dimensional problems, this procedure has to be repeated at several locations along the fracture edge (at every node when there is a possibility of branching). This makes the energy release rate criterion relatively computationally intensive. This is an important factor and plays a crucial role in choosing a suitable propagation criterion in numerical modeling of a hydraulic fracture.

\subsection{Fluid Flow in a Fracture}

Fluid flow in hydraulic fracturing treatments can be a complex problem in fluid mechanics which, in addition to the flow of a non-Newtonian fluid, involves phenomena of turbulence, leak-off, and variation of flow channel dimensions with time. Other factors adding to the complexity of the process are the difference in temperature between the fluid and the surrounding rock, change of fluid properties with temperature and flow, proppant transport and deposition. Most geothermal applications, however, do not involve complex fluid characteristics or variations of rheology with temperature, thus the Newtonian model is adequate. Newtonian fluids are defined as those exhibiting a direct proportionality between shear stress and shear rate in laminar flow, i.e.:

$$
\tau_{x y}=\mu \frac{d u}{d y}
$$

where $\mu$ is the viscosity. It is a proportionality constant independent of shear rate and is only a function of pressure and temperature in a given fluid system. Energy dissipation in Newtonian materials is by molecular collision. Therefore, all gases, liquids and solutions with low molecular weight are Newtonian. All those fluids for which the shear stress vs. shear rate relationship is non-linear through the origin, at a given pressure and temperature, are non-Newtonian.

Non-Newtonian fluids and can have complex constitutive equations. The simplest rheological model used for non-Newtonian fluids is the power-law model. This model has two constants, namely a power-law index, $n$, and a consistency index, $K$, and is written as [47]:

$$
\tau=K \gamma^{n}
$$

where $\gamma$ is the shear rate. If $n>1$, the fluid is dilatant; and if $n<1$, it is pseudoplastic. For power-law fluids one can define an apparent viscosity, $\mu_{a}$, analogous to viscosity of Newtonian fluids. It is computed 
using the relation:

$$
\mu_{a}=K(\gamma)^{n-1}
$$

The study of fluid rheology, in general, and viscoelasticity of crosslinked fluids specifically, need special attention and there is a need in the industry for viscoelastic characterization of fracturing fluid [7]. However, that pursuit is beyond the scope of the present study. Therefore, complex fluid behavior is not considered here.

A hydraulic fracture can be viewed as a channel of variable cross-section and height whose surfaces (fracture walls) are rough on both micro- and macro-scale. We assume that the rate of fluid injection is sufficiently small and the fluid viscosity is sufficiently large that the flow is laminar and the lubrication approximation can be applied to the fluid flow in the fracture. In lubrication approximation, the inertia terms can be neglected, and the applied pressure gradient is balanced by the laminar shear stresses. This is the same as the parallel plate flow model that is generally used to predict fluid flow in a hydraulic fracture and is also known as the Hele-Shaw model [18].

For laminar flow of a Newtonian fluid, the Navier-Stokes equations are:

$$
\rho \frac{D \mathbf{V}}{D t}=\rho \mathbf{F}-\nabla p+\mu \nabla^{2} \mathbf{V}+(\mu+\lambda) \nabla(\nabla \cdot \mathbf{V})
$$

where the material derivative is given by [22]:

$$
\frac{D}{D t}=\frac{\partial}{\partial t}+u \frac{\partial}{\partial x}+v \frac{\partial}{\partial y}+w \frac{\partial}{\partial z}
$$

And $\nabla^{2}$ is the Laplacian operator defined as:

$$
\nabla^{2}=\frac{\partial^{2}}{\partial x^{2}}+\frac{\partial^{2}}{\partial y^{2}}+\frac{\partial^{2}}{\partial z^{2}}
$$

and $\mathbf{V}$ is the velocity vector having the components $u, v$, and $w$ in the $x$-, $y$-, and $z$-directions, respectively. The fluid pressure is given by $p ; \mu$ is the fluid viscosity; $\lambda$ is the second viscosity coefficient, and $\mathbf{F}$ represents the body force vector with components $f_{x}, f_{y}, f_{z}$.

Assuming the fluid to be incompressible so that the rate of fluid volume dilation vanishes, i.e. $\nabla \cdot \mathbf{V}=0$, yields:

$$
\frac{D \mathbf{V}}{D t}=\rho \mathbf{F}-\nabla p+\mu \nabla^{2} \mathbf{V}
$$

Neglecting the effect of leak-off, the fluid has zero velocity (no slip condition) at the walls and at the top and bottom. Therefore, there is a three-dimensional velocity distribution. But, the width of the fracture, is much smaller than other (parallel-plate) dimensions (Figures 5 and 6).

As a result $w=0$ and the flow can be treated as a case of two-dimensional flow in the plane of the fracture. Then, for zero body forces, the flow equations become:

$$
\begin{aligned}
\mu \nabla^{2} u-\frac{\partial p}{\partial x} & =\rho\left(\frac{\partial u}{\partial t}+u \frac{\partial u}{\partial x}+v \frac{\partial u}{\partial y}\right) \\
\mu \nabla^{2} v-\frac{\partial p}{\partial y} & =\rho\left(\frac{\partial v}{\partial t}+u \frac{\partial v}{\partial x}+v \frac{\partial v}{\partial y}\right) \\
\frac{\partial p}{\partial z} & =0
\end{aligned}
$$

Due to the fact that the fluid velocity at the fracture wall equals zero, the change in the velocity components perpendicular to the wall is much greater than the changes along the fracture. Hence, the latter can be 


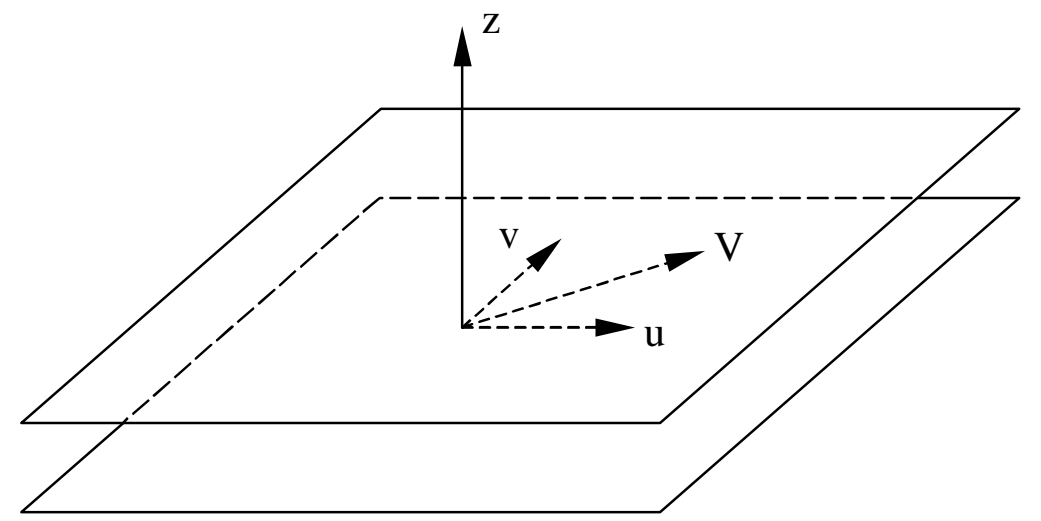

Figure 5: Paralle plate model for flow in a fracture.

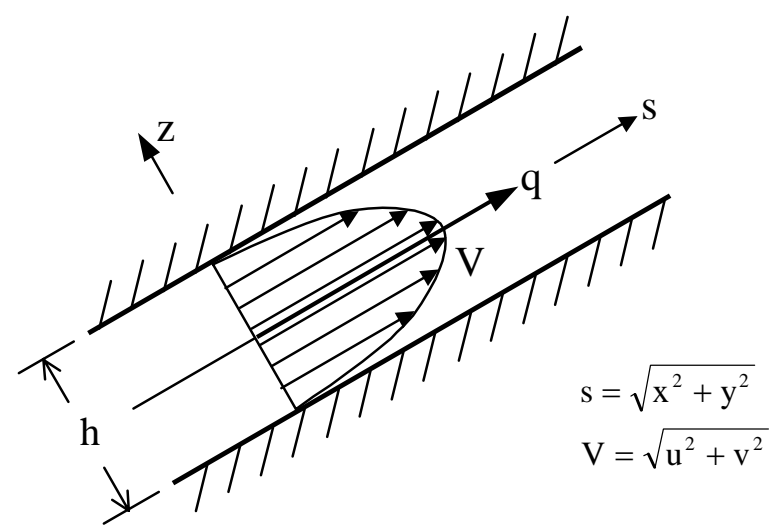

Figure 6: Velocity profile in a fracture. 
neglected, i.e., $\frac{\partial u}{\partial x}=\frac{\partial u}{\partial y}=\frac{\partial v}{\partial x}=\frac{\partial v}{\partial y}=0$. In addition for steady-state flow $\frac{\partial u}{\partial t}=\frac{\partial v}{\partial t}=0$, so that the equations reduce to:

$$
\begin{gathered}
\frac{\partial p}{\partial x}=\mu \frac{\partial^{2} u}{\partial z^{2}} \\
\frac{\partial p}{\partial y}=\mu \frac{\partial^{2} v}{\partial z^{2}} \\
\frac{\partial p}{\partial z}=0
\end{gathered}
$$

The last equation of the set indicates that the variation in pressure in the $z$ - direction is hydrostatic only, i.e., the total pressure at any point within the flow domain is a function of only the $x$ - and $y$-coordinates and is independent of $z$ [18]. Therefore, the above equations can be integrated with respect to $z$ to yield:

$$
\begin{aligned}
& u=\frac{z^{2}}{2 \mu} \frac{\partial p}{\partial x}+c_{1} z+c_{2} \\
& v=\frac{z^{2}}{2 \mu} \frac{\partial p}{\partial y}+c_{3} z+c_{4}
\end{aligned}
$$

The boundary conditions are given by $\frac{\partial u}{\partial z}=\frac{\partial v}{\partial z}=0$ at $z=0$ (from symmetry), and $u=v=0$ at $z=\frac{W}{2}$, where $W$ represents the width of the flow channel. The constants of integration are obtained by application of these boundary conditions which yields:

$$
\begin{array}{r}
c_{1}=c_{3}=0 \\
c_{2}=-\frac{W^{2}}{8 \mu} \frac{\partial p}{\partial x}=0 \\
c_{4}=-\frac{W^{2}}{8 \mu} \frac{\partial p}{\partial y}=0
\end{array}
$$

Therefore, the velocity components are described by:

$$
\begin{aligned}
& u=\frac{1}{2 \mu}\left(z^{2}-\frac{W^{2}}{4}\right) \frac{\partial p}{\partial x} \\
& v=\frac{1}{2 \mu}\left(z^{2}-\frac{W^{2}}{4}\right) \frac{\partial p}{\partial y}
\end{aligned}
$$

The average velocities over the distance $W$ between the plates are obtained by integration of velocities over that distance:

$$
\begin{aligned}
& u_{\text {avg }}=\frac{1}{W} \int_{\frac{-W}{2}}^{\frac{W}{2}} u d z=-\frac{W^{2}}{12 \mu} \frac{\partial p}{\partial x} \\
& v_{\text {avg }}=\frac{1}{W} \int_{\frac{-W}{2}}^{\frac{W^{2}}{2}} v d z=-\frac{W^{2}}{12 \mu} \frac{\partial p}{\partial y}
\end{aligned}
$$

Multiplication by cross-sectional area open to flow, $W b$, yields the flow rate:

$$
q_{i}^{\prime}=-\frac{b W^{3}}{12 \mu} p_{, i}
$$

Thus the flow rate per unit length is given by:

$$
q_{i}=-\frac{W^{3}}{12 \mu} p_{, i}
$$

where $i$ is the direction of flow and the comma denotes differentiation with respect to the $i$ - direction. The above equation is known as the cubic law.

Because the roughness of the fracture wall was not considered in the preceding derivation, the validity of theoretical results based on the parallel plate models have been questioned [49]; however, Wiles [48] has concluded that for typical fracture geometries (width greater than $1 \mathrm{~cm}$ ), the wall roughness effects can be neglected.

Implicit in the above model is the assumption that fluid leak-off and spurt loss do not influence the fluid flow behavior in the fracture. In order to include this influence, the flow model should account for flow 
perpendicular to the fracture wall so that the Hele-Shaw model is inadequate for this purpose. The fluid losses can have an important influence on the flow behavior if the magnitude of fluid loss is large enough, but, for small leak-off, normally the effect on parabolic velocity profile is insignificant [51].

The continuity (conservation of mass) equation for steady-state flow of an incompressible fluid through a channel of constant (in time) cross-section is:

$$
q_{i, i}^{\prime}=Q^{\prime}
$$

where $Q^{\prime}$ is a flow rate source per unit area of fracture surface (or sink) which represents the spurt loss, leak-off, and storage losses. Using this equation and Eq.(27), the following governing differential equation for steady-state laminar flow of a Newtonian fluid is obtained:

$$
k p_{, i i}+Q^{\prime}=0
$$

in which $k=\frac{-W^{3}}{12 \mu}$ is the conductivity coefficient.

It should be noted that the fracture aperture is a (non-linear) function of the effective normal stress, material properties, and its surface characteristics. Shear displacement of the fracture caused by stimulation, chemical dissolution, and rock cooling alters the aperture and thus the flow.

\subsection{Fluid Diffusion into the Formation (Leak-off)}

The fluid which leaks from the fracture into the rock formation is referred to as fluid loss. Fluid loss in geothermal systems can occur in preexisting cracks and pores or in new cracks that are formed as a result of cooling of the rock. In lieu of a more rigorous theoretical treatment; the fluid loss has been traditionally accounted for by considering two different contributing mechanisms, namely (i) storage loss and (ii) leak-off. Storage loss is the fluid that remains in the fracture and can be calculated using fracture width. The leak-off represents the losses by porous media flow, including flow into existing fractures.

As a first approximation, assuming that the fluid in the fracture has the same viscosity and compressibility as the reservoir fluid, the fluid leak-off is calculated using a one-dimensional steady-state potential flow perpendicular to the fracture wall. This is referred to as Carter's model of leak-off, which is usually written as [19]:

$$
q_{\ell}=\frac{C_{\ell}}{\sqrt{t}}
$$

where $q_{\ell}$ represents fluid flux. The pressure and other constants are lumped into $C_{\ell}$, the fluid loss coefficient. It is an experimentally determined constant that takes into consideration the build-up of a filter-cake on the fracture walls. This expression for leak-off was derived based on the assumption that the pressure at the fracture surface does not affect leak-off.

The fluid leak-off increases the pore pressure of the surrounding rock through a diffusion mechanism which introduces a domain-type coupling between fluid flow and solid deformation processes [125]. The above procedures for treatment of fluid leak-off are not coupled to rock deformation. Hence, the influence of fluid diffusion on fracture opening is not included. When a hydraulic fracture is modeled within the framework of the theory of poroelasticity [125], not only is leak-off coupled to deformation via the use of a poroelastic source solution but also, the leak-off coefficient emerges as a natural outcome of the theory in terms of fluid and solid material parameters. This poroelastic coupling will be briefly described later but is not included in the fracture propagation modeling. 


\subsection{Hear Transfer \& Heat Flow Model Development}

Although the focus of this project is rock mechanics issues and in particular hydraulic fracturing, the modeling of heat flow is necessary in order to quantify the impact of cooling on rock stresses. Furthermore, most heat extraction models in the literature are one-dimensional and robust three-dimensional models are not available. The ancillary benefit of the work is development of reliable and optimum energy production plan.

The thermal energy from enhanced geothermal systems is extracted from the reservoir by water circulation. Heat extraction is controlled by conduction through the intact rock blocks towards the water conducting fractures whose width may have been increased by stimulation. Fluid flow rates in the fractures are strongly dependant on fracture aperture. As indicted before, the fluid velocity is proportional to the square of the fracture width (for a given pressure gradient) which itself varies with hydraulic, thermo-mechanical, and chemical factors. These processes are intrinsically coupled and their interaction needs to be studied numerically. But, the chemical aspects are beyond the scope of this project that addresses two other important aspects of enhanced geothermal systems namely fracture propagation and the thermal disturbance induced by hydraulic flow in terms of its impact on fluid temperature and reservoir stress. As a result, we will investigate heat extraction from a fracture by allowing two- and three-dimensional heat flow in the rock mass.

It should be emphasized that in modeling stimulation by hydraulic fracturing, we treat the hydromechanical aspects explicitly while neglecting the thermo-mechanical effects due to heat transfer. This is justified because, as will be shown, the thermal stresses develop very slowly in comparison to fracture propagation. Also, when treating the circulation problem, we consider the fracture geometry and fluid velocity to be known a priori from a previous step or independent analyses, and treat the thermoelastic coupling explicitly. To close the loop, one would need to use the calculated thermal stresses to determine the new fracture geometry and fluid velocity in the fracture. This, however, is not treated herein and will have to be completed in the next phase of the project.

In treating the heat transfer problem in the context of the circulation operations, we first re-examine the traditional solution to the problem of heat extraction from a two-dimensional fracture [81, 85]. Subsequently, the influence of heat storage and heat dispersion terms in the heat transport equation within the fracture are considered and their effects investigated. Furthermore, we investigate the role of fluid loss on extraction temperature using a one-dimensional leak-off model. Then, the solution to the problem of heat extraction from a fracture is considered using a two-dimensional heat flow model in the rock matrix. The predicted water temperature is compared with that obtained from a conventional one-dimensional approach to heat flow in the rock mass. Then, the resulting thermal stresses are calculated using the $2 \mathrm{D}$ solution. Finally, we consider and numerically solve the three-dimensional heat conduction effect in hot dry rock. The numerical difficulty of modeling a three-dimensional, unbounded domain is overcome by utilizing the integral equation formulation and the three-dimensional Green's function of heat conduction. The three-dimensional heat conduction effect is then investigated against its one-dimensional simplification.

In should be noted that in studying heat transfer and extraction we will neglect fluid viscosity/temperature coupling, density driven convection, and effects of transient flow.

\subsubsection{Mathematical formulation for 1D heat flow}

In developing an advanced 3D model for the heat extraction problem, a quasi-two-dimensional heat conduction model is examined first $[81,85]$. This solution is not fully two-dimensional because the heat conduction in 
the reservoir is restricted to the direction perpendicular to the fracture surface. In revisiting and reformulating this problem, we make the following commonly accepted assumptions for a fracture in a two-dimensional reservoir [85]:

1. The reservoir is of constant thickness and infinite horizontal extent, and is insulated at the top and the bottom (see Figure 7)

2. The fracture is vertical and penetrates the entire thickness of the reservoir. The curvature of its trajectory on the horizontal plan is negligible

3. All properties, which include the fracture thickness, permeability, reservoir heat capacity, etc., are constant

4. The injection rate of cold water through a vertical well intersecting the fracture is steady. The reservoir is impermeable to water and the fracture has no storage capacity; hence the production rate of hot water is equal to the injection rate

5. In the fracture, the heat transport by advection and dispersion takes place only in the longitudinal direction

6. In the rock mass, heat conduction takes place only in the direction perpendicular to the fracture (see Figure 8). However, this one-dimensional heat flow assumption will be relaxed in the next stage of solution.

The governing equations for the quasi-2D model are:

Equation for heat transport in the fracture:

$$
b \rho_{w} c_{w}\left(\frac{\partial T_{w}}{\partial t}+v \frac{\partial T_{w}}{\partial x}-D_{L} \frac{\partial^{2} T_{w}}{\partial x^{2}}\right)=\left.K_{r} \frac{\partial T_{r}}{\partial y}\right|_{y=0}
$$

where $T_{w}=T_{w}(x, t)$ is the water temperature in the fracture, $T_{r}=T_{r}(x, y, t)$ is the rock temperature, $b$ is the half-thickness of the fracture, $\rho_{w}$ is the water density, $c_{w}$ is the specific heat of water, $v=Q / 2 b$ is the average velocity of water in the fracture, $Q$ is the injection flow rate per unit height of fracture, $D_{L}$ is the longitudinal dispersion coefficient, and $K_{r}$ is the rock thermal conductivity. The first term on the left represents the rate of change of temperature in a control volume (transient heat storage in the fluid); the second term represents advective heat transport; and the third term denotes the dispersive heat transport.

Equation for heat conduction in the rock:

$$
\frac{\partial^{2} T_{r}}{\partial y^{2}}=\frac{\rho_{r} c_{r}}{K_{r}} \frac{\partial T_{r}}{\partial t}
$$

where $\rho_{r}$ is the rock density, and $c_{r}$ is the specific heat of rock. The fracture is idealized to be straight; however, in a fracture model having a small curvature, the $x$-direction would be a measurement along the fracture trajectory, and the $y$-direction would be normal to it (Figure 7). Mathematically, the geometry of the system under consideration is shown in Figure 8.

The initial temperature in the rock is considered to be a constant, $T_{r}=T_{r o}$, as is the initial temperature of water in the fracture, $T_{w}=T_{r o}$. At the injecting point, $x=0$, the injected water is at a constant 


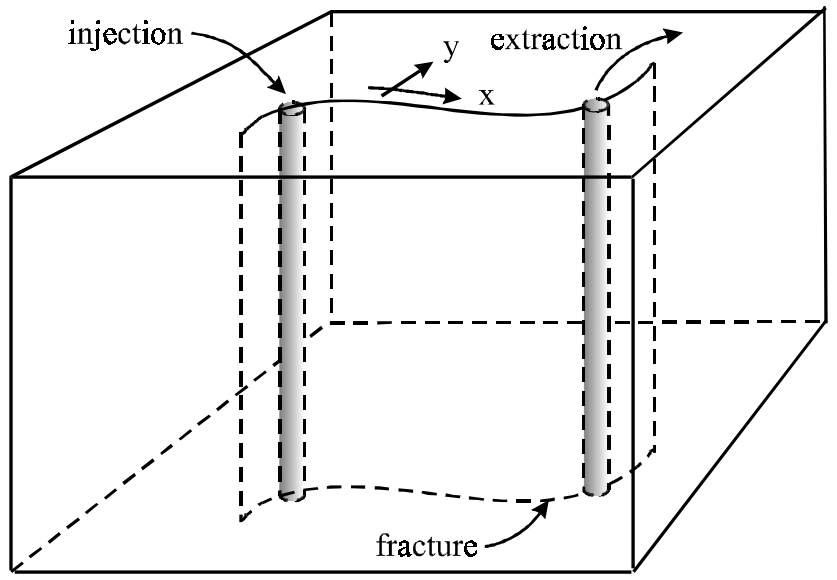

Figure 7: Idealized view of heat extraction from a hot dry rock reservoir, 1D model.

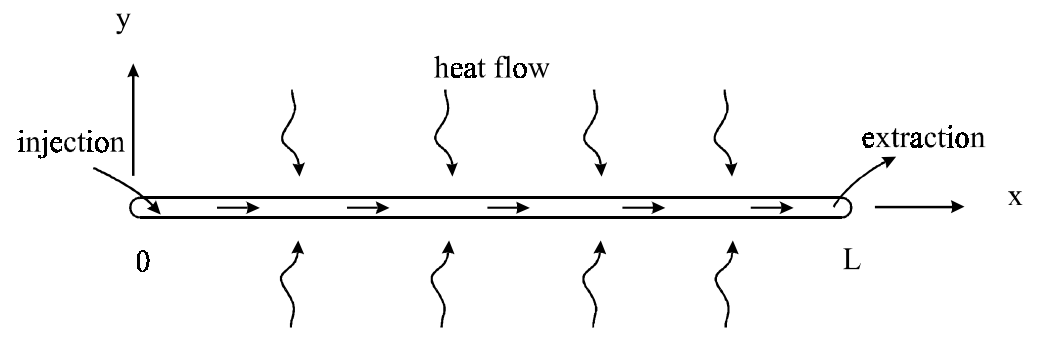

Figure 8: Mathematical model for heat extraction from a fracture. 


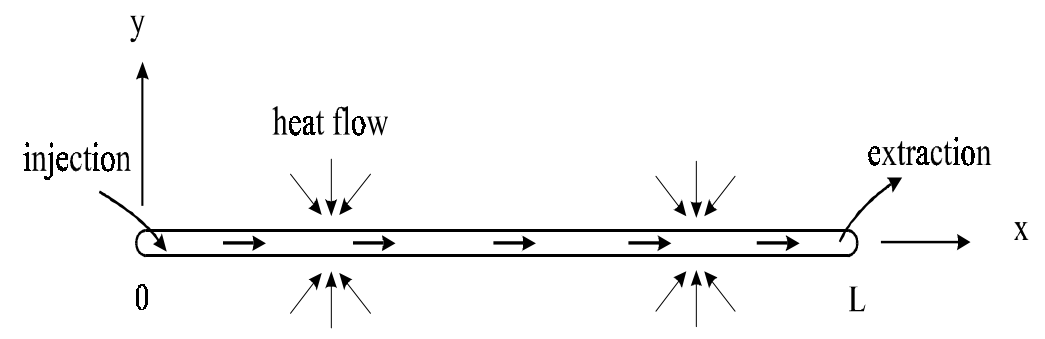

Figure 9: Geometry of two-dimensional heat extraction from a reservoir.

temperature $T_{w o}$. These two equations, (31) and (32), are to be solved with the above initial and boundary conditions.

The above models have common deficiency: the heat flow in the reservoir is assumed to be onedimensional, perpendicular to the fracture face. Indeed, this is a common assumption for the majority of hot dry rock heat extraction models. Next, we relax this assumption and include the effect of full twodimensional heat flow is included. Then, we compare the results with the one-dimensional model in order to assess the impact of this simplification on water temperature.

\subsubsection{Mathematical model for 2D heat flow}

Figure 9 defines the geometry of the mathematical problem defined below.

The governing equations for the two-dimensional model are:

For heat transport in the fracture:

$$
b \rho_{w} c_{w} v \frac{\partial T_{w}}{\partial x}=\left.K_{r} \frac{\partial T_{r}}{\partial y}\right|_{y=0^{+}} \quad \text { for } 0 \leq x \leq L
$$

where $T_{w}=T_{w}(x, t)$ is the water temperature in the fracture, $T_{r}=T_{r}(x, y, t)$ is the rock temperature in the reservoir, $b$ is the half-thickness of the fracture, $\rho_{w}$ is the water density, $c_{w}$ is the specific heat of water, $v=Q / 2 b$ is the average velocity of water, $Q$ is the water injection rate per unit height of fracture, $K_{r}$ is the rock thermal conductivity, and $L$ is the fracture length. In the above we have neglected the heat storage and dispersion terms based on earlier findings [80] that showed their contributions to be negligible under most practical conditions.

For heat conduction in the rock:

$$
K_{r}\left(\frac{\partial^{2} T_{r}}{\partial x^{2}}+\frac{\partial^{2} T_{r}}{\partial y^{2}}\right)=\rho_{r} c_{r} \frac{\partial T_{r}}{\partial t} \quad \text { for }-\infty \leq x, y \leq+\infty
$$

where $\rho_{r}$ is the rock density, and $c_{r}$ is the specific heat of rock. The left side of the above equation indicates that the heat flow in the reservoir is two-dimensional. For comparison, the Lowell [81] model assumes a one-dimensional heat flow:

$$
K_{r} \frac{\partial^{2} T_{r}}{\partial y^{2}}=\rho_{r} c_{r} \frac{\partial T_{r}}{\partial t} \quad \text { for } 0 \leq x \leq L \quad \text { and } \quad-\infty \leq y \leq+\infty
$$


The above governing equations, (33) and (34), are subject to initial and boundary conditions. The initial temperature of the rock and the water in fracture is at a constant,

$$
\begin{gathered}
T_{r}(x, y, 0)=T_{r o} \\
T_{w}(x, 0)=T_{r o}
\end{gathered}
$$

prior to the injection of cold water. At the injecting point, $x=0$, the injected water is at a different constant temperature

$$
T_{w}(0, t)=T_{w o}
$$

On the fracture face, the reservoir temperature equals the water temperature:

$$
T_{r}(x, 0, t)=T_{w}(x, t)
$$

\subsubsection{Mathematical model for 3D heat flow}

A number of investigators have noted the importance of three-dimensional heat flow in the rock. However, no robust analytical solution for the case a finite fracture exists. Moreover, most numerical solutions that been developed for the case of a finite fracture use the finite element method. We consider and numerically solve the three-dimensional heat conduction problem for finite and infinite fractures in hot dry rock. The numerical difficulty of modeling a three-dimensional, unbounded domain is overcome by utilizing the integral equation formulation and the three-dimensional Green's function of heat conduction. The need for discretizing the reservoir is entirely eliminated, and the final numerical solution system involves only the two-dimensional fracture plane, resulting in a much more efficient numerical scheme. Figure 10 illustrates a schematic view of heat extraction from a hot dry rock system by circulating water through a natural or man-made fracture. The fracture is assumed to be flat, of finite size, and arbitrary shape. The geothermal reservoir, on the other hand, is of infinite extent.

Other physical assumptions are similar to these postulated in [108]. Specifically, it is assumed that the geothermal reservoir is impermeable to water and is non-deformable, and has constant heat conduction properties. The heat storage and dispersion effects in the fracture fluid flow are negligible. We further postulate that the fracture width is small such that the flow in the fracture is laminar and governed by the lubrication flow equation:

$$
\nabla_{2} p(x, y)=-\frac{\pi^{2} \mu}{w^{3}(x, y)} \mathbf{q}(x, y) ; \quad x, y \in A
$$

where $\nabla_{2}$ is the gradient operator in two spatial dimensions ( $x$ and $\left.y\right), p$ is the fluid pressure, $\mu$ the fluid viscosity, $w$ the fracture width, and $A$ the fracture surface (see Figure 10). We note that:

$$
\mathbf{q}=w \overline{\mathbf{v}}
$$

is the discharge per unit width, and $\overline{\mathbf{v}}$ is the average flow velocity given by:

$$
\overline{\mathbf{v}}(x, y)=\frac{1}{w(x, y)} \int_{0}^{w(x, y)} \mathbf{v}(x, y, z) d z
$$




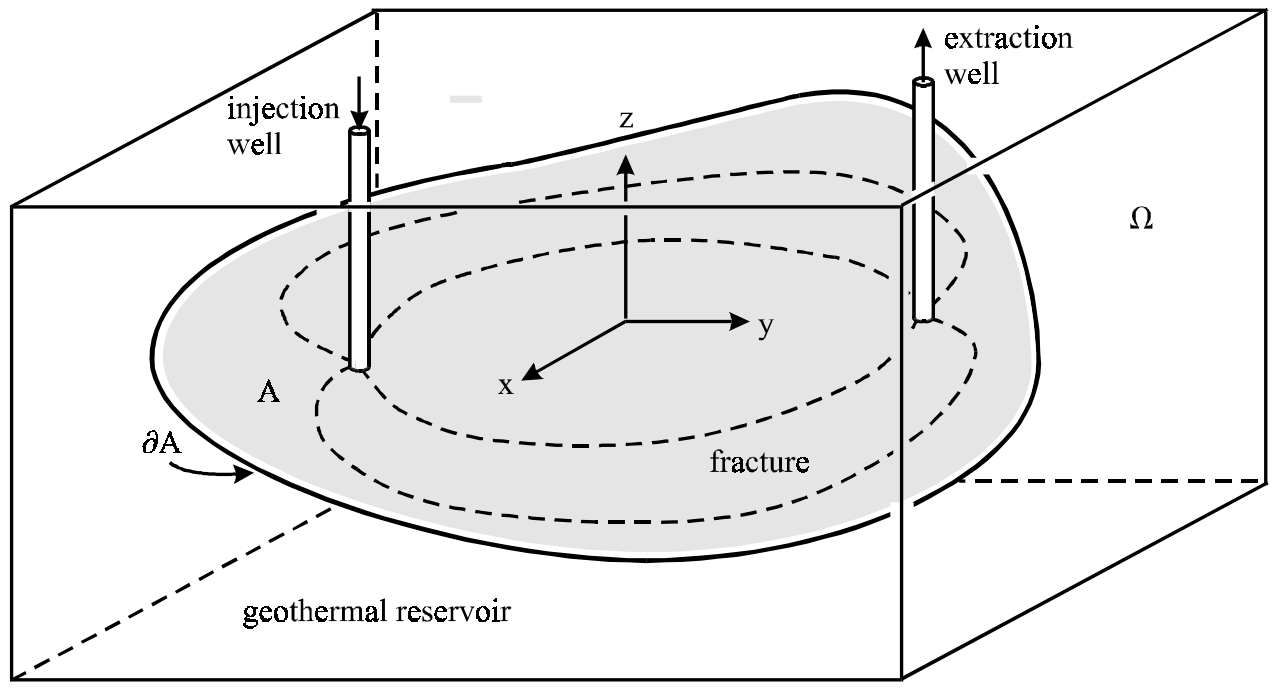

Figure 10: Heat extraction from a plane fracture.

where $\mathbf{v}$ is the flow velocity.

Assuming that the fluid is incompressible, the reservoir is impermeable to fluid flow, and the fracture width does not change with time, we can write the fluid continuity equation as:

$$
\nabla_{2} \cdot \mathbf{q}(x, y)=Q \delta\left(x-x_{e}, y-y_{e}\right)-Q \delta\left(x-x_{i}, y-y_{i}\right)
$$

where $\nabla_{2}$. is the divergence operator in two-dimension. In the above we have considered an injection well located at $\left(x_{i}, y_{i}\right)$, and an extraction well at $\left(x_{e}, y_{e}\right)$, both of discharge $Q$; and $\delta$ is the Dirac delta function.

Combining (31) and (43), we obtain the following second order partial differential equation

$$
\nabla_{2} \cdot\left[w^{3}(x, y) \nabla_{2} p(x, y)\right]=\pi^{2} \mu Q\left[\delta\left(x-x_{i}, y-y_{i}\right)-\delta\left(x-x_{e}, y-y_{e}\right)\right]
$$

which is subject to the boundary condition

$$
\frac{\partial p}{\partial n}=0 \quad \text { on } \partial A
$$

where $\partial A$ is the fracture boundary (Figure 10), and $n$ is the outward normal of $\partial A$. With known fracture width, the above equation can be solved for the pressure distribution in the fracture. The discharge $\mathbf{q}$ and average velocity $\overline{\mathbf{v}}$ are then obtained from (40) and (41).

Heat Transport The heat transport takes place both in the geothermal reservoir and the fracture. For the geothermal reservoir, the heat conduction is governed by the three-dimensional diffusion equation:

$$
K_{r} \nabla_{3}^{2} T(x, y, z, t)=\rho_{r} c_{r} \frac{\partial T(x, y, z, t)}{\partial t} ; \quad x, y, z \in \Omega
$$

where $T$ is the temperature, $\rho_{r}$ is the rock density, $c_{r}$ is the specific heat of rock, $\nabla_{3}^{2}$ is the Laplacian operator in three-dimensions, and $\Omega$ represents the infinite domain that is the geothermal reservoir. 
For heat transport in the fracture, in addition to the heat storage and diffusion terms as found in (46), we also need to consider advection by fluid flow and the heat source effects. However, under the condition of relatively large advection velocity and the continuous injection of cooling water, it can be demonstrated that the heat storage and diffusion effects are negligible [108, 81]. Dropping these terms, the governing equation becomes

$$
\begin{gathered}
\rho_{w} c_{w} \nabla_{2} \cdot[\mathbf{q}(x, y) T(x, y, 0, t)]=\left.2 K_{r} \frac{\partial T(x, y, z, t)}{\partial z}\right|_{z=0^{+}} \\
+\rho_{w} c_{w} Q\left[T\left(x_{e}, y_{e}, 0, t\right) \delta\left(x-x_{e}, y-y_{e}\right)\right. \\
\left.-T_{i n j} \delta\left(x-x_{i}, y-y_{i}\right)\right] ; \quad x, y \in A
\end{gathered}
$$

where $\rho_{w}$ is the water density, $c_{w}$ is the specific heat of water, $K_{r}$ is the rock thermal conductivity, and $T_{i n j}$ is the injection water temperature. In the above, the term on the left hand side of (47) represents the heat advection by fracture fluid flow, the first term on the right hand side gives the heat supply through the fracture walls (two sides) by conduction, and the last two terms correspond to a heat sink and a heat source, caused by the extraction and injection of water. We note that in (47) a single notation $T(x, y, z, t)$ is used to denote the temperature of both the rock and the fracture fluid, because temperature is continuous across the two media. The water temperature $T_{w}$ is equal to the rock temperature on the fracture plane $z=0$, i.e., $T_{w}(x, y, t)=T(x, y, 0, t), x, y \in A$.

Equation (47) can be simplified by expanding the quantities under the divergence operator and utilizing the flow equation (43). It becomes:

$$
\rho_{w} c_{w} \mathbf{q}(x, y) \cdot \nabla_{2} T(x, y, 0, t)-\left.2 K_{r} \frac{\partial T(x, y, z, t)}{\partial z}\right|_{z=0^{+}}=0
$$

We notice that the Dirac delta functions are removed from the equation.

The governing equations (46) and (48) are subject to initial and boundary conditions. The temperature of the rock and the fracture fluid before the heat extraction operation is assumed to be a constant,

$$
T(x, y, z, 0)=T_{o} ; \quad x, y, z \in \Omega
$$

At the injection point $\left(x_{i}, y_{i}, 0\right)$, the temperature is equal to that of the injected water:

$$
T\left(x_{i}, y_{i}, 0, t\right)=T_{i n j}
$$

The extraction temperature $T\left(x_{e}, y_{e}, 0, t\right)$ is unknown. It is of interest to note that there is no explicit boundary or interfacial condition for the geothermal reservoir. The heat flux and temperature continuity at the interface with the fracture is automatically contained in the coupling term in (48) and the inherent assumption of a single temperature for the entire system. The only boundary value introduced to the system is the injection temperature shown in (50).

\subsection{Coupled Poro-Thermoelastic Processes}

As discussed previously, poromechanical, thermal, and chemical processes are active when developing a geothermal reservoirs. These processes occur on various time scales and the significance of their interaction and coupling is dependent upon the problem of interest. For example during drilling operations there is a strong coupling between thermal and poromechanical effects that has significant impact of the stress/pore 
pressure distribution around the wellbore and thus hole failure (fracture initiation). This is because usually the time required for drilling a length equal to five times the hole's radius is much smaller than the characteristic time $\frac{a^{2}}{c^{f}}$, where $a$ is the radius of the well and $c^{f}$ is the fluid diffusivity. On the other hand during the stimulation by hydraulic fracturing, the evolution of the fluid-rock mechanics coupling evolves rapidly (on the scale of minutes, hours to possibly days) compared to thermal processes, thus the thermal effects have little effect on the fluid-mechanical processes involved in fracture propagation. However, During the circulation phase (time scale of years), the thermo-mechanical coupling can no longer be neglected. In fact, thermo-mechanical processes are also coupled to chemical processes. Accordingly, different levels of coupling are considered herein when studying wellbore failure, fracture propagation, and fluid circulation. Below the theoretical framework for coupling poromechanical and thermal phenomena are presented and their significance in various problems of interest are described.

When fluid saturated rocks are heated (cooled), the bulk solid as well as the pore fluid tend to undergo expansion (contraction). The volumetric expansion can result in significant pressurization of the pore fluid depending on the degree of containment and the thermal and hydraulic properties of the fluid as well as the solid. The net effect is a coupling of the thermal and poromechanical processes. The poro-thermomechanical response of fluid saturated porous rocks plays an important role in wellbore failure and fracture initiation, insitu stress measurements, and injection/production aspects of geothermal energy production. These effects are accounted for in the theory of porothermoelasticity.

The isothermal theory of poroelasticity couples pore pressure and solid stress fields in deformable fluid saturated porous rocks [119]. The time dependent fluid flow is incorporated by combining the fluid mass conservation with Darcy's law; and the basic constitutive equations relate the total stress to both the effective stress given by deformation of the rock matrix and the pore pressure arising from the fluid. A poro-thermoelastic approach combines the theory of heat conduction with poroelastic constitutive equations, coupling the temperature field with the stresses and pore pressure. The governing equations of poro-thermoelasticity are [126]-[127]:

Constitutive equation:

$$
\begin{gathered}
\Delta \varepsilon_{i j}=\frac{\Delta \sigma_{i j}}{2 G}, \quad i \neq j \\
\Delta \varepsilon_{k k}=\frac{\Delta \sigma_{k k}}{3 K}+\frac{\alpha \Delta p}{K}+\beta_{s}^{\prime} \Delta T \\
\sigma_{i j}=2 G \varepsilon_{i j}+\frac{2 G \nu}{1-2 \nu} \varepsilon \delta_{i j}-\alpha p \delta_{i j}+K \beta_{s}^{\prime} T \delta_{i j} \\
\Delta \zeta=\frac{\alpha}{3 K} \Delta \sigma_{k k}+\frac{\alpha \Delta p}{B K}-\phi_{0}\left(\beta_{f}-\beta_{s}^{\prime \prime}\right) \Delta T
\end{gathered}
$$

Equilibrium equations: 


$$
\sigma_{i j, j}=0
$$

Darcy's law:

$$
v_{i}=-\frac{k}{\mu} p_{, i}
$$

Continuity equations:

$$
\frac{\partial \zeta}{\partial t}+q_{i, j}=0
$$

Fourier:

$$
q_{i}^{T}=-k^{T} T_{, i}
$$

where $\sigma_{i j}$ denotes the components of the total stress tensor, $\zeta$ is the variation of the fluid content per unit volume of the porous material, and $\varepsilon_{i j}$ are the components of the strain tensors related to the solid displacements $u_{i}$ by $\varepsilon_{i j}=\frac{1}{2}\left(u_{i, j}+u_{j, i}\right)$. The constant $K$ is the rock's bulk modulus; $B$ is Skempton's pore pressure coefficient; $\alpha=\frac{3\left(\nu_{u}-\nu\right)}{B(1-2 \nu)\left(1+\nu_{u}\right)}$ is Biot's effective stress coefficient; $\beta_{s}^{\prime}$ is the volumetric thermal expansion coefficient of the bulk solid under constant pore pressure and stress; and $\beta_{s}^{\prime \prime}$ and $\beta_{f}$ represent volumetric thermal expansion coefficients of the solid matrix and the pore fluid, respectively. The former, $\beta_{s}^{\prime \prime}$, reflects the phenomena engendered by the internal pore geometry and stress fields that are caused by a temperature change; it may be considered equal to $\beta_{s}^{\prime}$ (henceforth denoted by $\beta_{s}$ ) if the change in temperature is not expected to change porosity, $\phi_{o} . \kappa=\frac{k}{\mu}$, in which $k$ is dynamic permeability and $\mu$ is the fluid viscosity; $k^{T}$ is the thermal conductivity and $c^{T}$ is the thermal diffusivity. The above equations can be combined to yield a set of field equations:

$$
\begin{gathered}
G \nabla^{2} \mathbf{u}+\left(\frac{G}{1-2 \nu}\right) \boldsymbol{\nabla}(\boldsymbol{\nabla} \cdot \mathbf{u})-\alpha \boldsymbol{\nabla} \mathbf{p}-\beta_{s} K \boldsymbol{\nabla} T=\mathbf{0} \\
\frac{1}{M} \frac{\partial p}{\partial t}=\frac{k}{\eta} \nabla^{2} p-\alpha \frac{\partial(\boldsymbol{\nabla} \cdot \mathbf{u})}{\partial t}+\left[\alpha \beta_{s}+\phi\left(\beta_{f}-\beta_{s}\right)\right] \frac{\partial T}{\partial t} \\
\frac{\partial T}{\partial t}=c^{T} \nabla^{2}
\end{gathered}
$$

in which $G$ is shear modulus and $M=\frac{2 G\left(\nu_{u}-\nu\right)}{\alpha^{2}(1-2 \nu)\left(1-2 \nu_{u}\right)}$ is Biot's modulus. It should also be noted that for most rocks, heating/cooling produces thermal stresses and changes pore pressure, but stress and pressure changes do not significantly alter the temperature field so that the latter is not coupled to the equations for the pore pressure and solid displacements. Also, note that convective heat transport is neglected. This is justified for most rocks of interest as they are well represented by a low permeability system. 
In order to consider the influence of a temperature gradient on both pore pressure and stresses, it is necessary to use the above non-isothermal poroelastic theory, or poro-thermoelasticity. However, many problems formulated within the framework of poro-thermoelasticity are not amenable to analytical treatment and need to be solved numerically. This is accomplished here using a boundary element method. 


\section{Mathematical Methods \& Numerical Algorithms}

In this section we present details of mathematical methods and certain aspects of the numerical algorithms involved in various models and solutions. We also present some examples that will show the validity of the analytical and numerical solutions. Additional examples are presented to highlight the various physical processes and their interactions.

\subsection{Poro-Thermoelastic Boundary Element Method}

As indicated before, many problems formulated within the framework of poro-thermoelasticity need to be solved numerically. This is accomplished here using a boundary element method. The BEM scheme for porothermoelasticity is described in Appendix D. The validity of the numerical approach for porothermoelastic problems has been demonstrated in [125] where the numerical model has been compared to analytical results; it has been shown that the numerical results are in very good agreement with the analytic solution. Some example are presented here to highlight the coupled effects in problems of concern in geothermal reservoir development.

\subsubsection{Wellbore failure and fracture initiation}

The stress distribution around a wellbore is an important concern in drilling and hydraulic fracturing operations for production of geothermal energy. Consider a borehole with radius $R=0.1 \mathrm{~m}$ in a reservoir (Figure 11) at a temperature of $200^{\circ} \mathrm{C}$ (fluid/rock parameters are shown in the above Table). The formation is assumed to be Westerly Granite, with the major principle stress, $\sigma_{H}=25 \mathrm{MPa}$, minor principle stress, $\sigma_{h}=20 \mathrm{MPa}$, and pore pressure, $p=10 \mathrm{MPa}$. The wellbore wall is suddenly cooled by water and maintained at $80^{\circ} \mathrm{C}$. For clarity of presentation and investigation of the role of temperature, only induced stresses and pore pressure are studied and the far field pore pressure and stresses are considered to be zero. Due to symmetry only a quarter of the wellbore is modeled using 10 elements. For each computation, the time is divided into 10 steps with the value of increment adjusted accordingly.

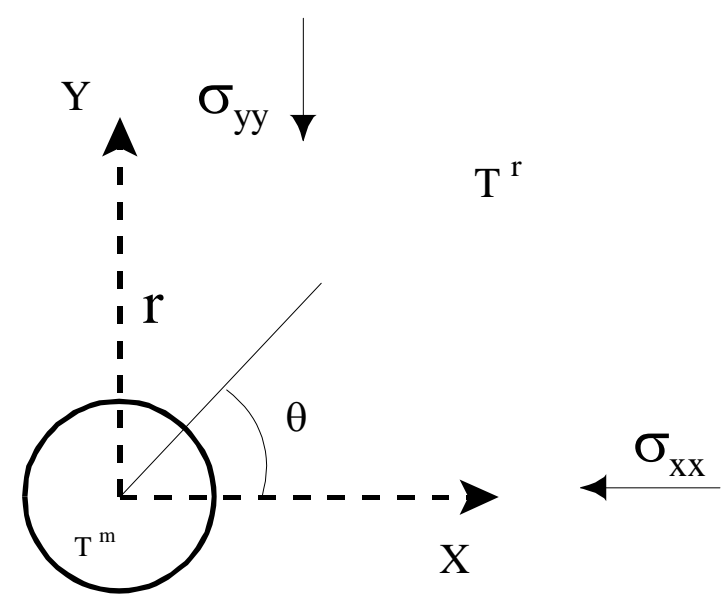

Figure 11: Problem geometry and boundary conditions for a circular borehole in an infinite porous medium. 
Temperature around a circular borehole

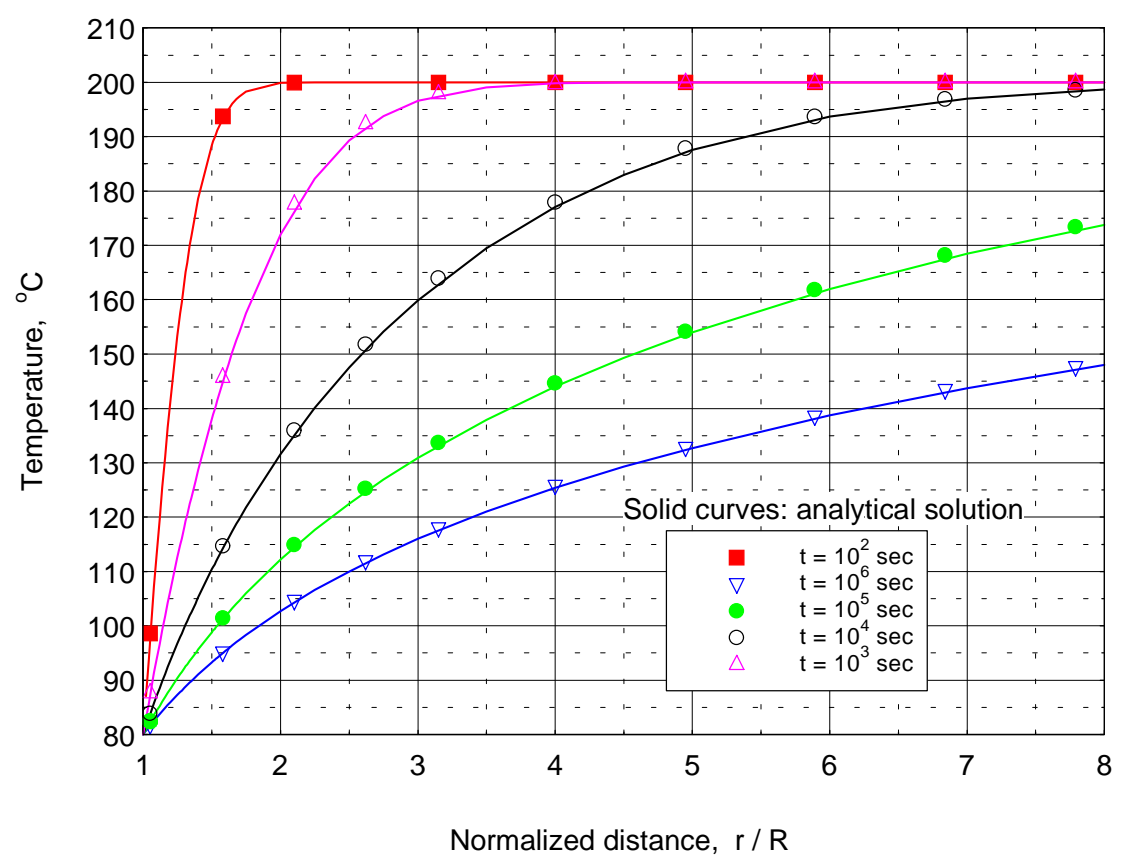

Figure 12: Temperature distribution around the wellbore.

\begin{tabular}{|l|l|l|l|}
\hline \multicolumn{3}{|l|}{ Table 1. Input Parameters } \\
\hline$E$ & elastic modulus & $3.75 \times 10^{04}$ & $\mathrm{MPa}$ \\
\hline$\nu$ & Poisson's ratio & 0.25 & \\
\hline$\nu_{u}$ & undrained Poisson's ratio & 0.34 & \\
\hline$K_{s}$ & solid bulk modulus & $4.50 \times 10^{04}$ & $\mathrm{MPa}$ \\
\hline$K_{f}$ & fluid bulk modulus & $2.50 \times 10^{03}$ & $\mathrm{MPa}$ \\
\hline$C$ & heat capacity & 790.0 & $\mathrm{Joule} /\left(\mathrm{kg} \cdot{ }^{\circ} \mathrm{C}\right)$ \\
\hline$c^{*}$ & thermal diffusivity & $5.10 \times 10^{-06}$ & $\mathrm{~m}^{2} / \mathrm{sec}$ \\
\hline$c^{f}$ & fluid diffusivity & $6.48 \times 10^{-05}$ & $\mathrm{~m}^{2} / \mathrm{sec}$ \\
\hline$\beta_{s}$ & solid thermal expansivity & $2.40 \times 10^{-05}$ & $\mathrm{~m} /{ }^{\circ} \mathrm{C}$ \\
\hline$\beta_{f}$ & fluid thermal expansivity & $3.00 \times 10^{-04}$ & $\mathrm{~m} /{ }^{\circ} \mathrm{C}$ \\
\hline$\phi_{0}$ & porosity & 0.01 & \\
\hline$k$ & dynamic permeability & $4.053 \times 10^{-08}$ & $\mathrm{~m} / \mathrm{sec}$ \\
\hline$B$ & Skempton's coefficient & 0.85 & \\
\hline$\mu$ & fluid viscosity & $3.55 \times 10^{-04}$ & $\mathrm{~kg} /(\mathrm{m} \cdot \mathrm{sec})$ \\
\hline
\end{tabular}

Figures 12-15 illustrate the profiles of induced temperature, pore pressure, radial, and tangential stress around the wellbore. It can be observed in Figure 12 that the transient temperature distribution is typical of conductive heat transfer. The formation is gradually cooled when the borehole temperature is suddenly reduced. Figure 13 shows that a significant pressure drop is generated near the borehole at early times. With time, the pore pressure peak is reduced and moves away from the well where it gradually recovers the original state. Figure 14 presents the induced radial stress, note that a significant radial tensile stress peak 


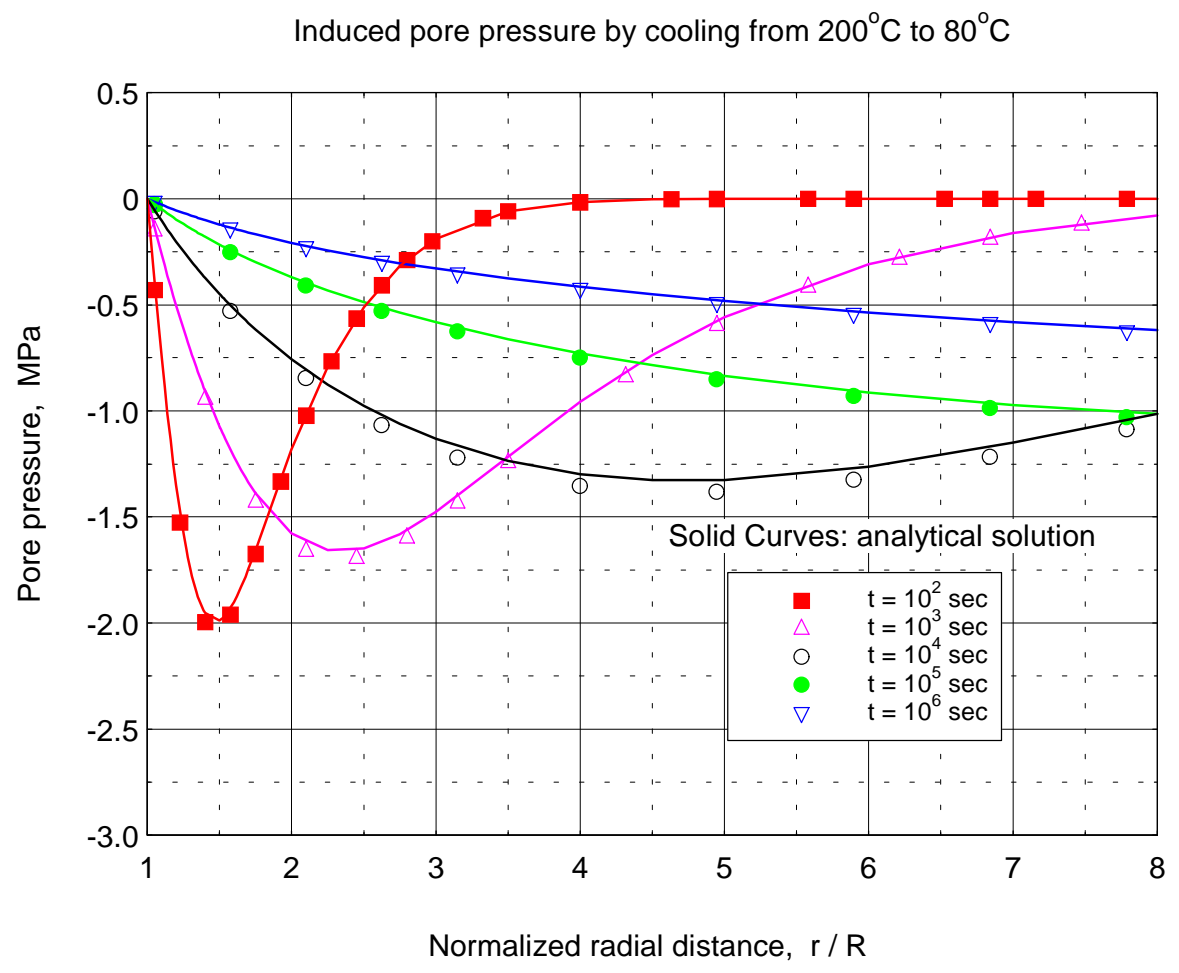

Figure 13: Cooling induced pore pressure around the wellbore. 
Induced radial stress by cooling from $200^{\circ} \mathrm{C}$ to $80^{\circ} \mathrm{C}$

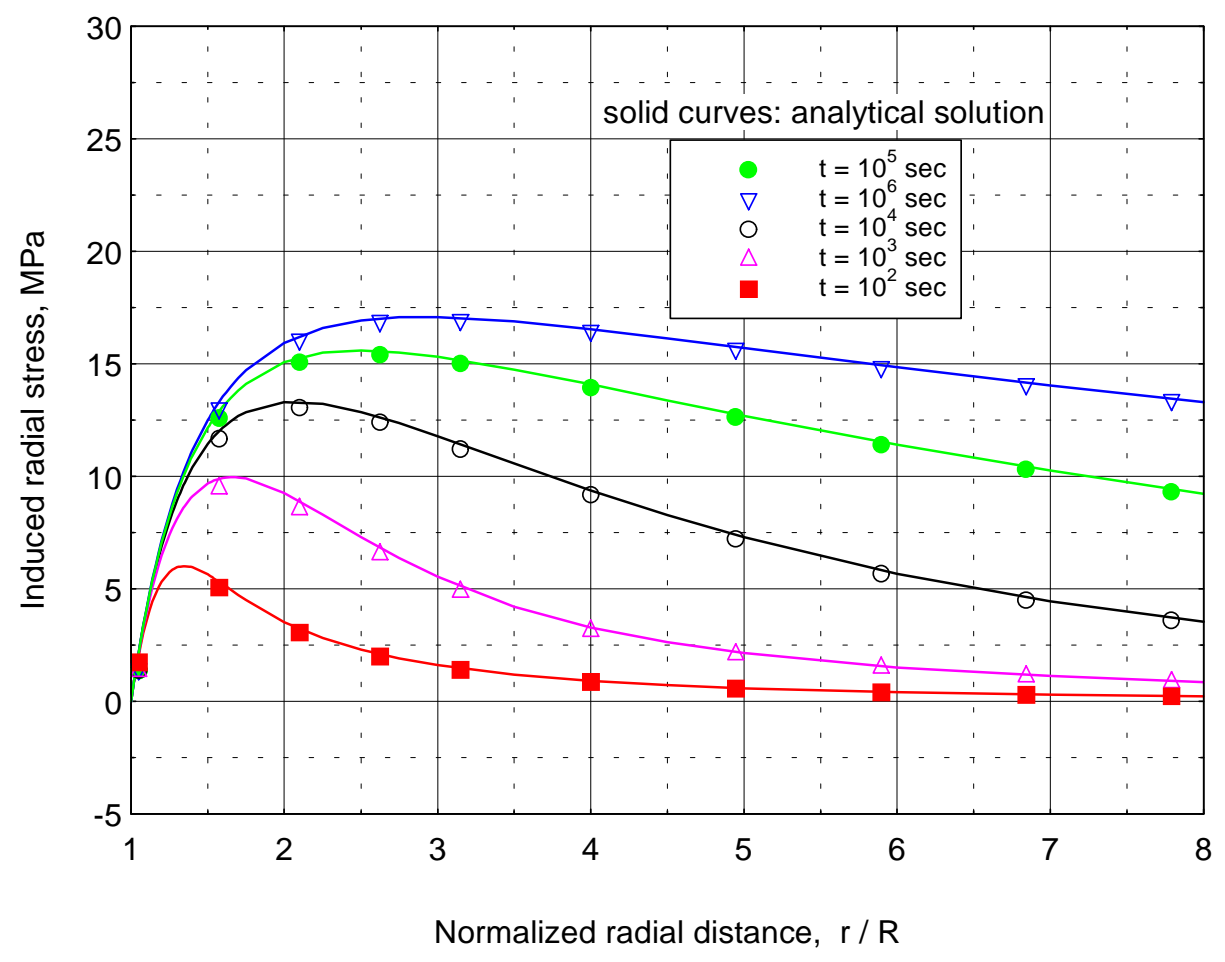

Figure 14: Cooling induced radial stress around the wellbore. 


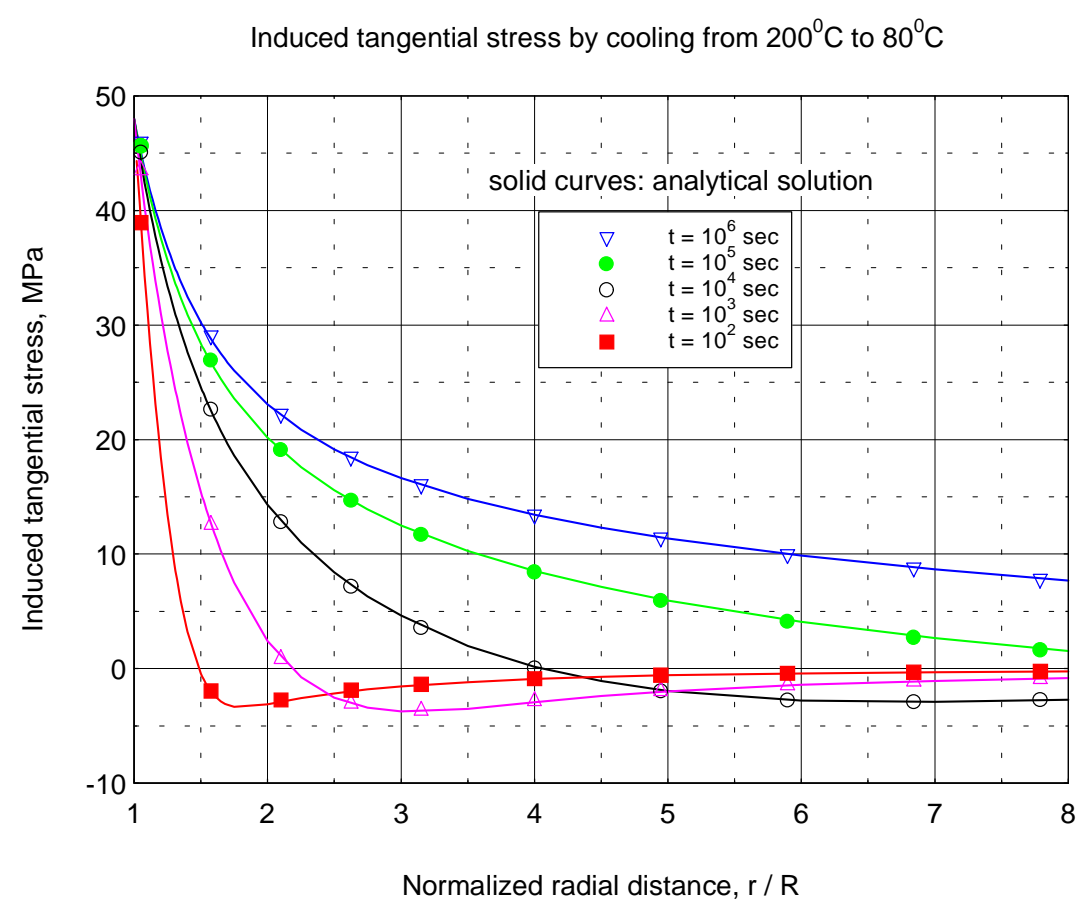

Figure 15: Cooling induced tangential stress around the wellbore. 


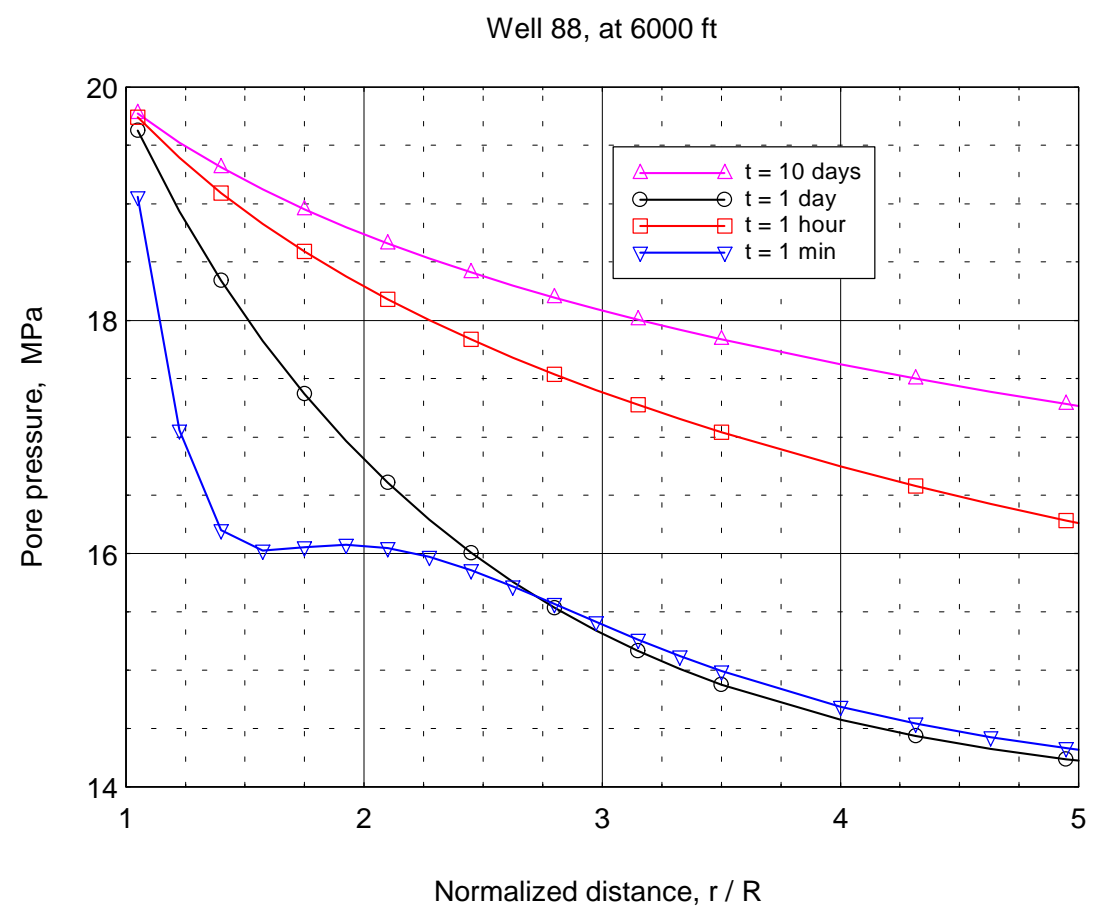

Figure 16: Total pore pressure around the wellbore. 
Well 88, at $6000 \mathrm{ft}$

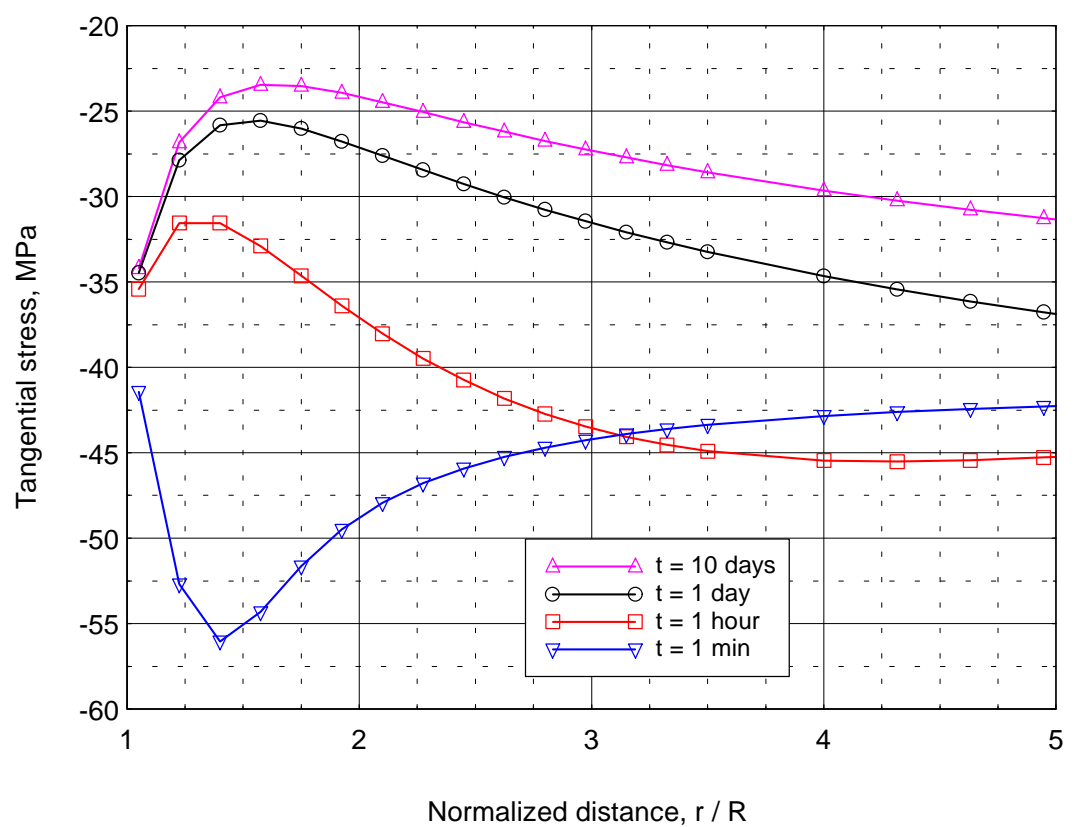

Figure 17: Distribution of tangential stress in the formation. 
Well 88, at $6000 \mathrm{ft}$

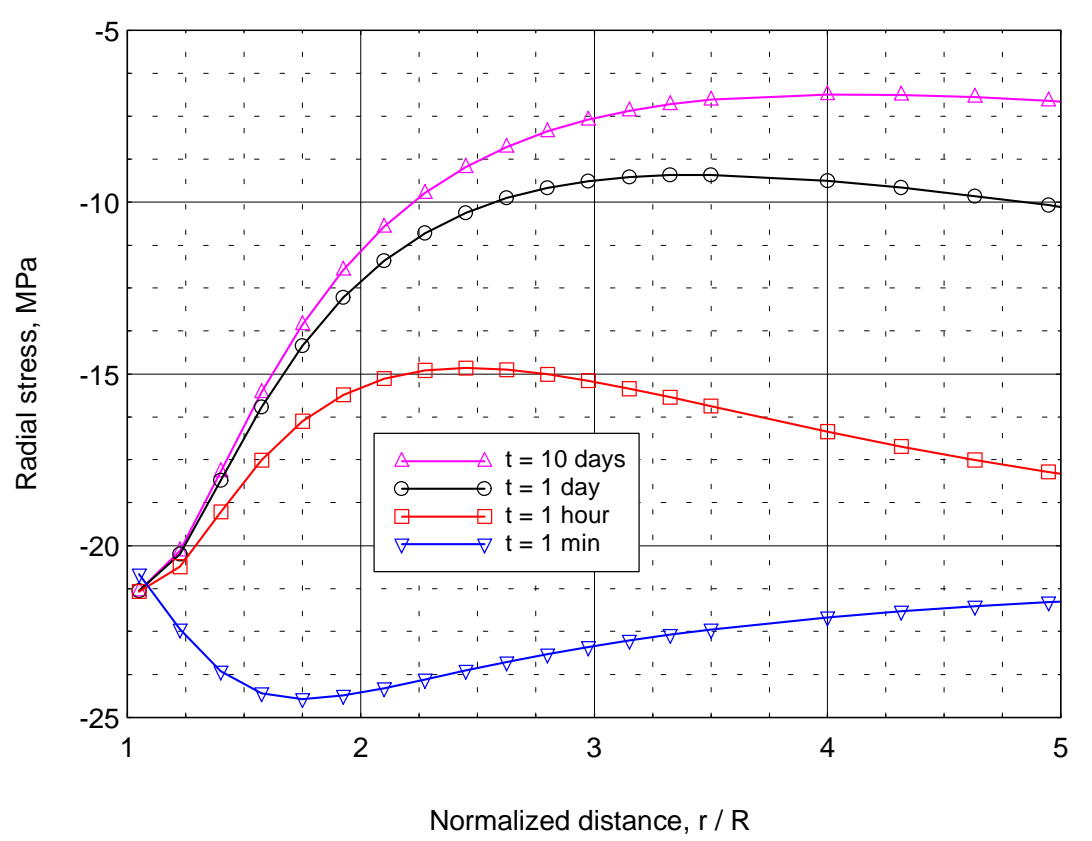

Figure 18: Distribution of radial stress around the wellbore.

is produced inside the formation. With time, the tensile stress zone moves inside the formation while the magnitude of the "peak" increases. Figure 15 presents the thermally induced tangential stress; the tensile tangential stress is induced around the wellbore due to the tendency of the rock to shrink near the borehole wall. Away from the borehole wall, the magnitude of the induced tensile stress decreases and changes its sign at some point inside the formation, i.e., it becomes compressive. This is because the shrinkage of the material near the borehole due to cooling tends to pull on the outer rock thus inducing a compressive stress zone that gradually moves away from the borehole. Note that although the maximum tensile (tangential) stress always occurs at the wall, a zone of tension also develops and extends into the formation with increasing cooling time. With time, the tensile radial stress zone moves inside the formation while the magnitude of the "peak" increases. Again, note that the zone of tension propagates into the rock and enhances fracturing.

The total pore pressure, tangential, and radial stress (the sum of the induced and in-situ stress) distribution for $\theta=0$ (direction of $S_{\min }$ in-situ stress) is shown in Figures 16, 17, and 18, respectively. These results are for Well \#88 in the Coso field (at a depth of $6000 \mathrm{ft}$ ). Note the high compressive stresses that can lead to wellbore breakouts. The tangential stress for $\theta=90$ illustrated in Figure 19. It can be observed that the high tensile tangential stresses are developed around the well as a result of cooling and in-situ stress concentration. Cooling causes the tensile zone to expand into the reservoir with time resulting in fracture initiation and propagation. Thus, one can take advantage of thermal stresses to stimulate geothermal reservoirs. 
Tangential stress field, $\theta=90^{\circ}$, tension ( + )

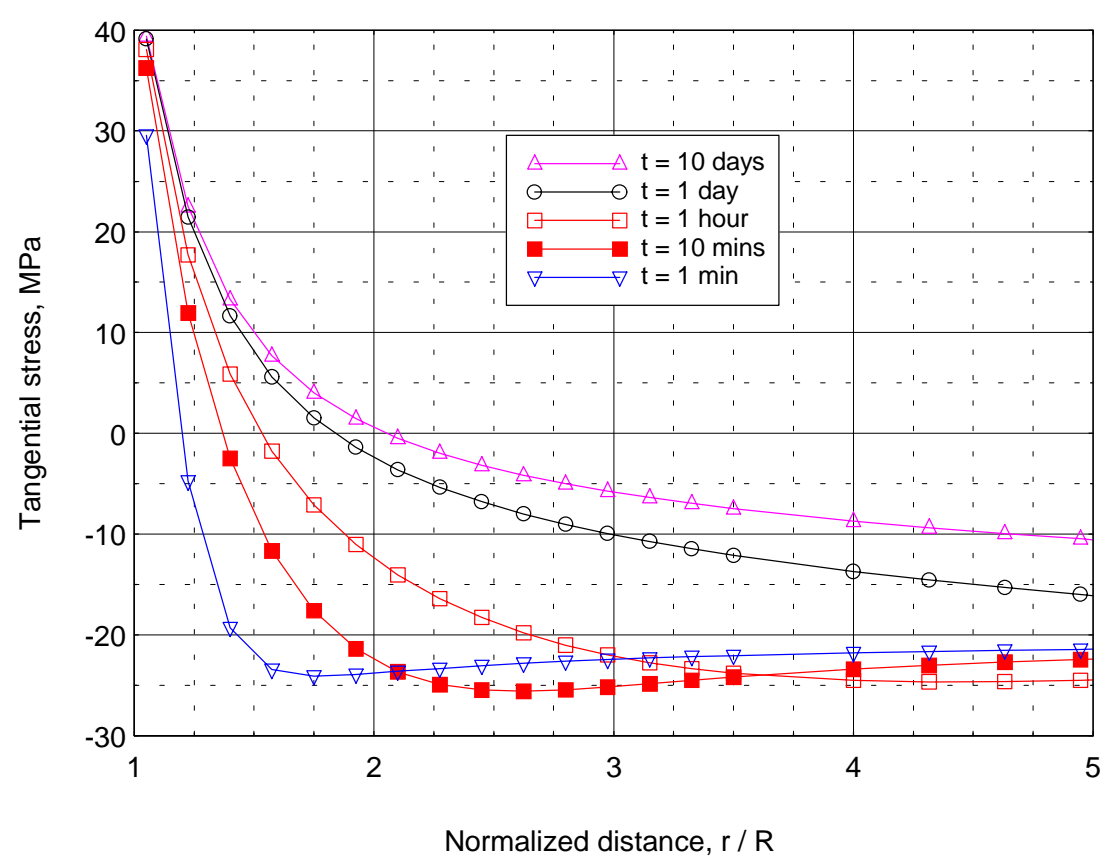

Figure 19: Tangential stress in the vicinity of the well, in the $S_{\max }$ direction. 


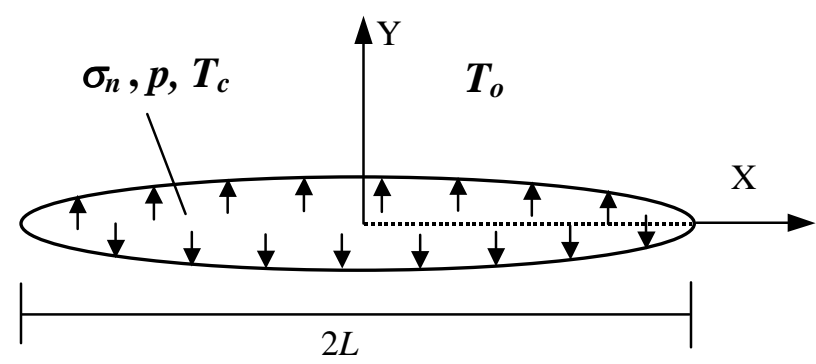

Figure 20: Uniformly pressurized, cooled crack.

\subsubsection{Fracture opening \& propagation}

The application of the fictitious stress boundary element method to wellbore stress analysis was described in the previous section. In this section, we apply a porothermoelastic DD method to the problem of a stationary crack in a geothermal reservoir. The results will be used to examine the relative importance of various mechanisms in fracture propagation.

The problem under consideration is the opening of a suddenly pressurized crack of length $2 L$ (shown in Figure 20). This problem of fracture pressurization by a cooler fluid under pressure P may be decomposed into three sub-problems corresponding to three fundamental modes of loading: a normal stress loading, a pore pressure loading, and a temperature loading:

$$
\begin{array}{ll}
\text { Mode 1: } & \sigma_{n}(x, t)=P H(t) ; p(x, t)=0 ; T(x, t)=0 \\
\text { Mode 2: } & p(x, t)=P H(t) ; \quad \sigma_{n}(x, t)=0 ; T(x, t)=0 \\
\text { Mode 3: } & T(x, t)=T_{1} H(t) ; \quad \sigma_{n}(x, t)=0 ; p(x, t)=0
\end{array}
$$

where $H(t)$ denotes the Heaviside step function. The initial conditions for each sub-problem are zero stress, pore pressure, and a temperature of $T_{0}$ everywhere. We will focus on the induced quantities with the aim of investigating various mechanisms.

The result of Mode 1 loading is shown in Figure 21. This mode of loading is responsible for the opening of the fracture. At time $t=0^{+}$, the fracture opens according to the well known solution (Sneddon, 1946) with undrained material properties:

$$
W=-\frac{2 p L\left(1-\nu_{u}\right)}{G}\left(1-\frac{x^{2}}{L^{2}}\right)
$$

As time increases the crack opening also increases, approaching the steady-state solution given by the previous equation with drained material properties. This stage of rock deformation is referred to as the drained stage. The crack opening is a maximum at this stage reflecting the softer material behavior. Figure 22 shows the normalized width evolution (with respect to Eq. 62) as a function of normalized time as defined by:

$$
\tau=\frac{t c^{f}}{L^{2}}
$$




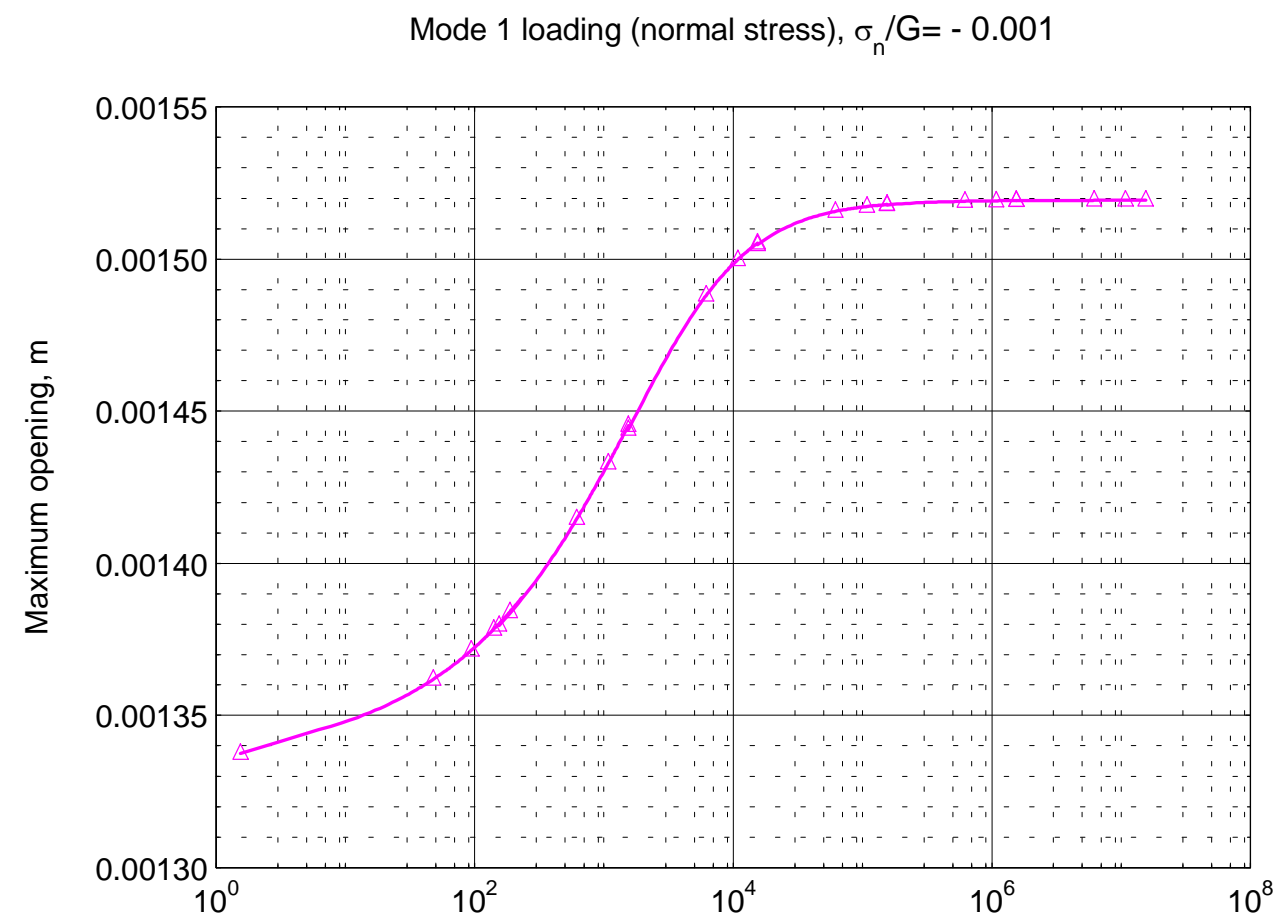

Time, sec

Figure 21: Magnitude of maximum crack opening as a fucntion of time for mode 1. 


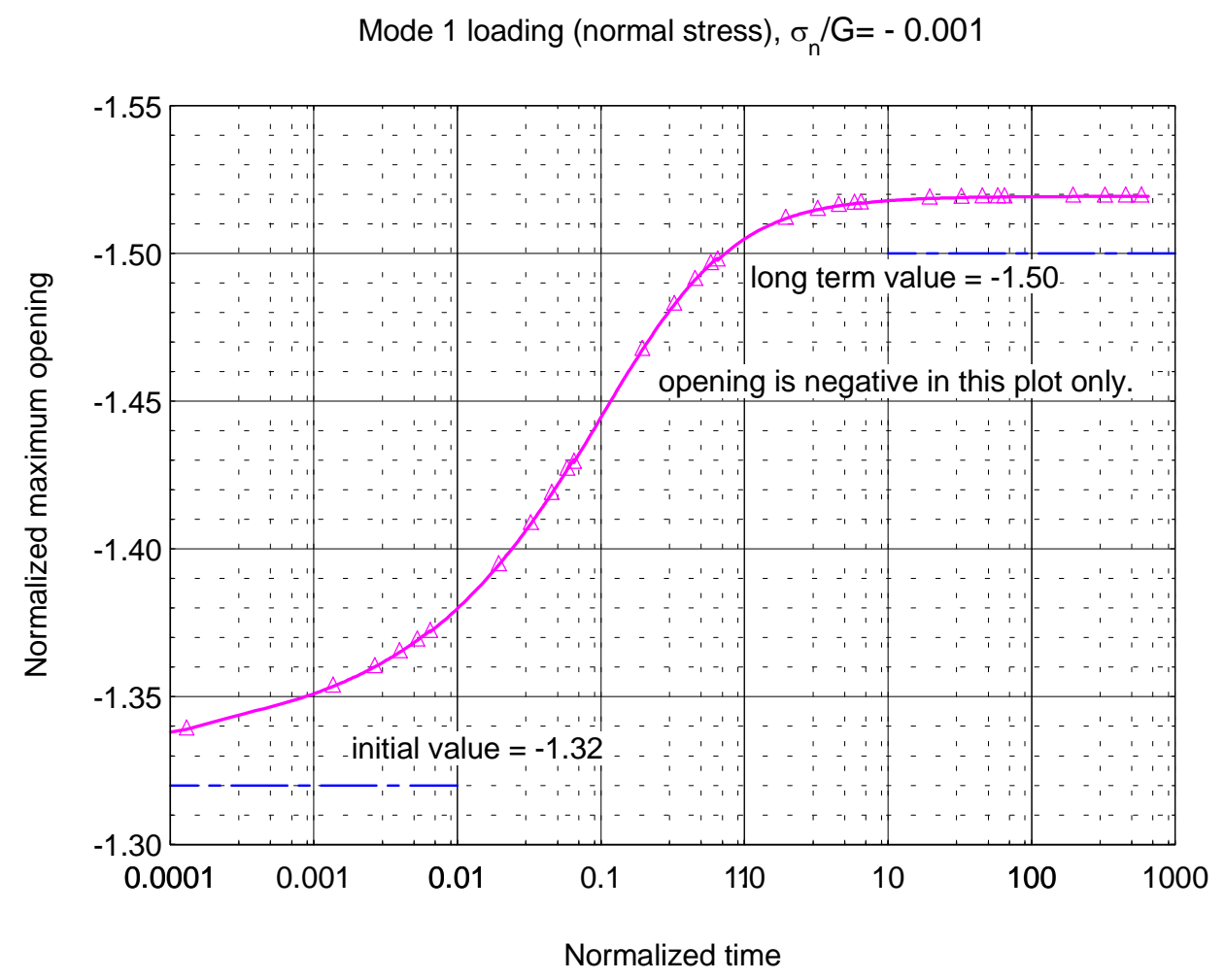

Figure 22: Normalized crack opening for mode 1. 
Mode 2 loading (pore pressure), $P / G=0.001$

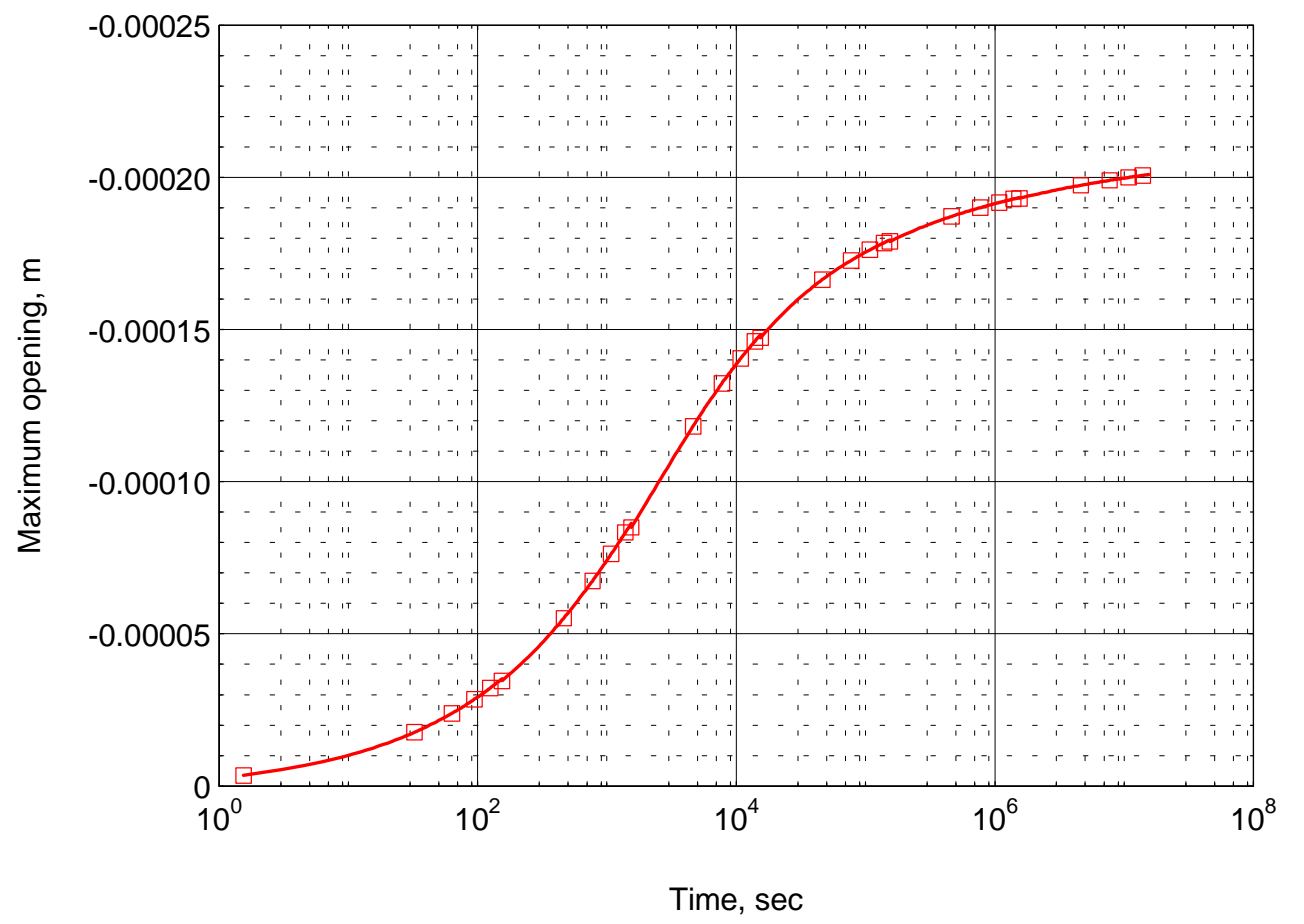

Figure 23: Magnitude of crack opening due to an applied pore pressure.

The fracture response in Mode 2 is illustrated in Figure 23. The crack closes progressively, starting from a zero value and reaching a final closure value of 0.22 given by:

$$
\left(\hat{D}_{n}\right)_{\max }=2 \eta(1-\nu)
$$

where $\eta$ is the poroelastic stress parameter defined as (Vandamme et al., 1989):

$$
\eta=\frac{3\left(\nu_{u}-\nu\right)}{2 B\left(1+\nu_{u}\right)(1-\nu)}
$$

Because the two crack surfaces cannot overlap, this closure is physically possible only if the crack remains open, under appropriate combinations of loading modes 1,2, and 3. The numerical model overestimates the fracture opening by about 5 per cent. This may be attributed to the used of constant elements. A comparable error was also observed when modeling pressurized cracks in elastic media using constant elements [119].

The Mode 3 fracture response as a function of time is illustrated in Figure 25. It can be seen that the cooling the crack surfaces and the surrounding rock results in opening of the fracture. This is the opposite of the effect of fluid invasion into the rock mass that tends to close the crack. 


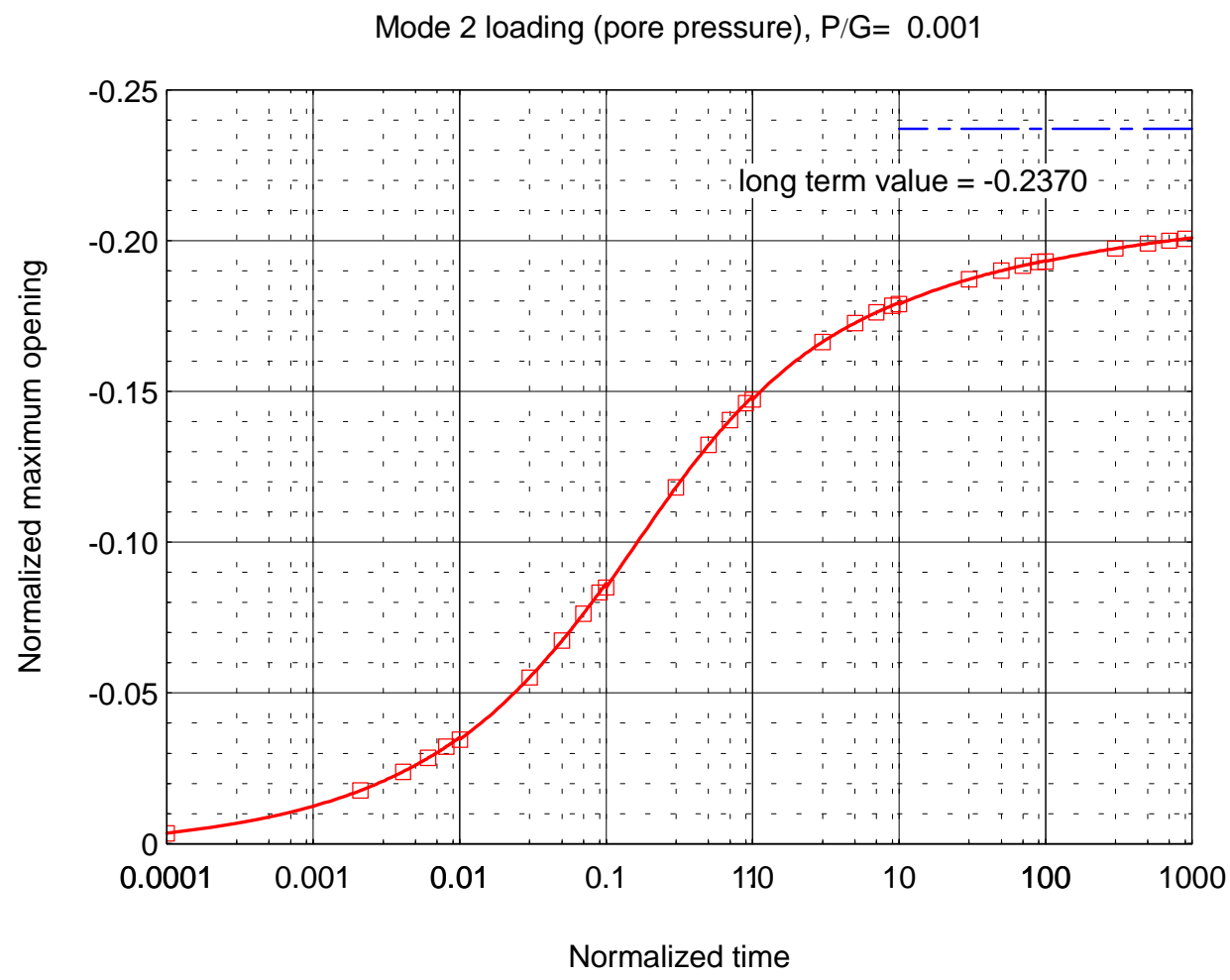

Figure 24: Normalized crack opening due to pore pressure loading. 


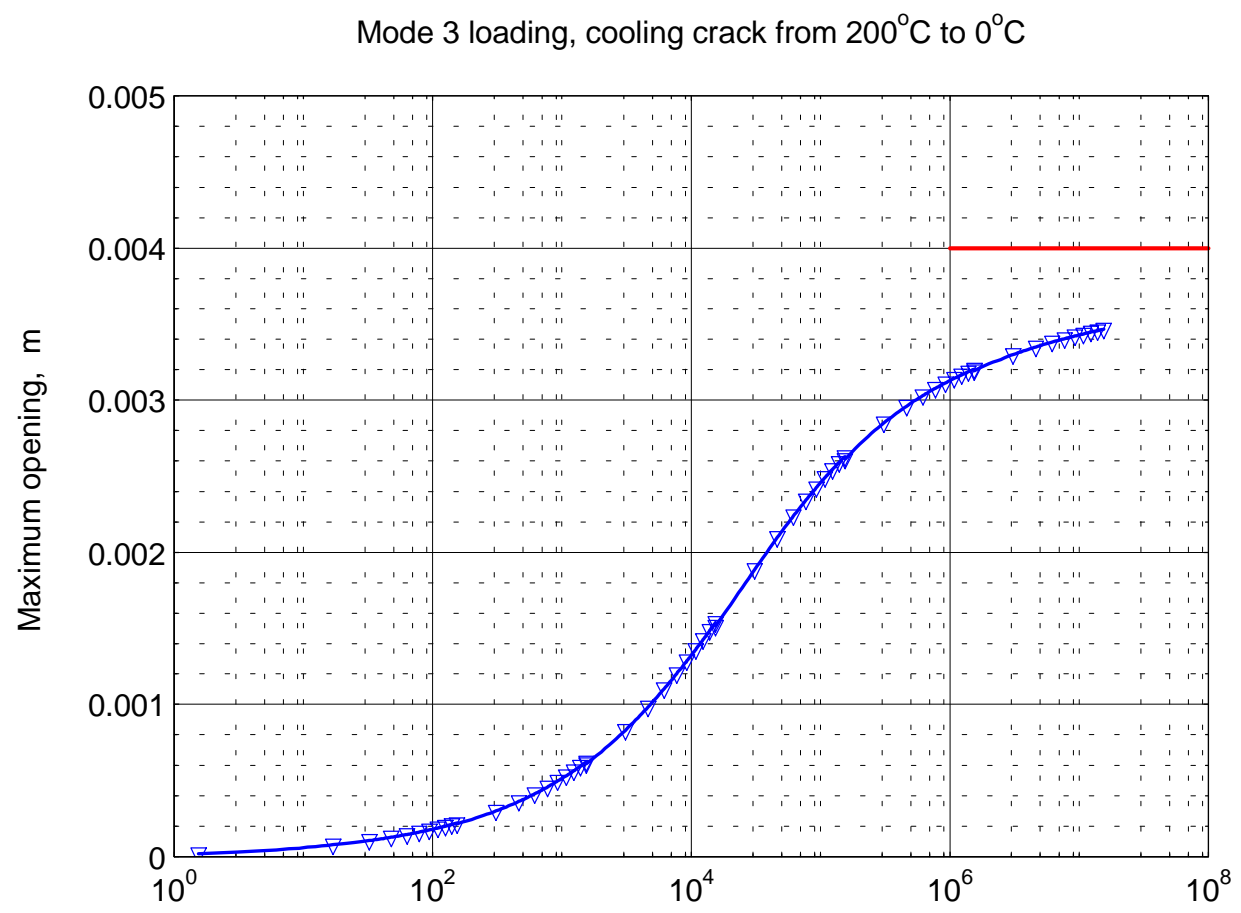

Time, sec

Figure 25: Maximum crack opening due to cooling, also shown is the steady-state analytical value. 


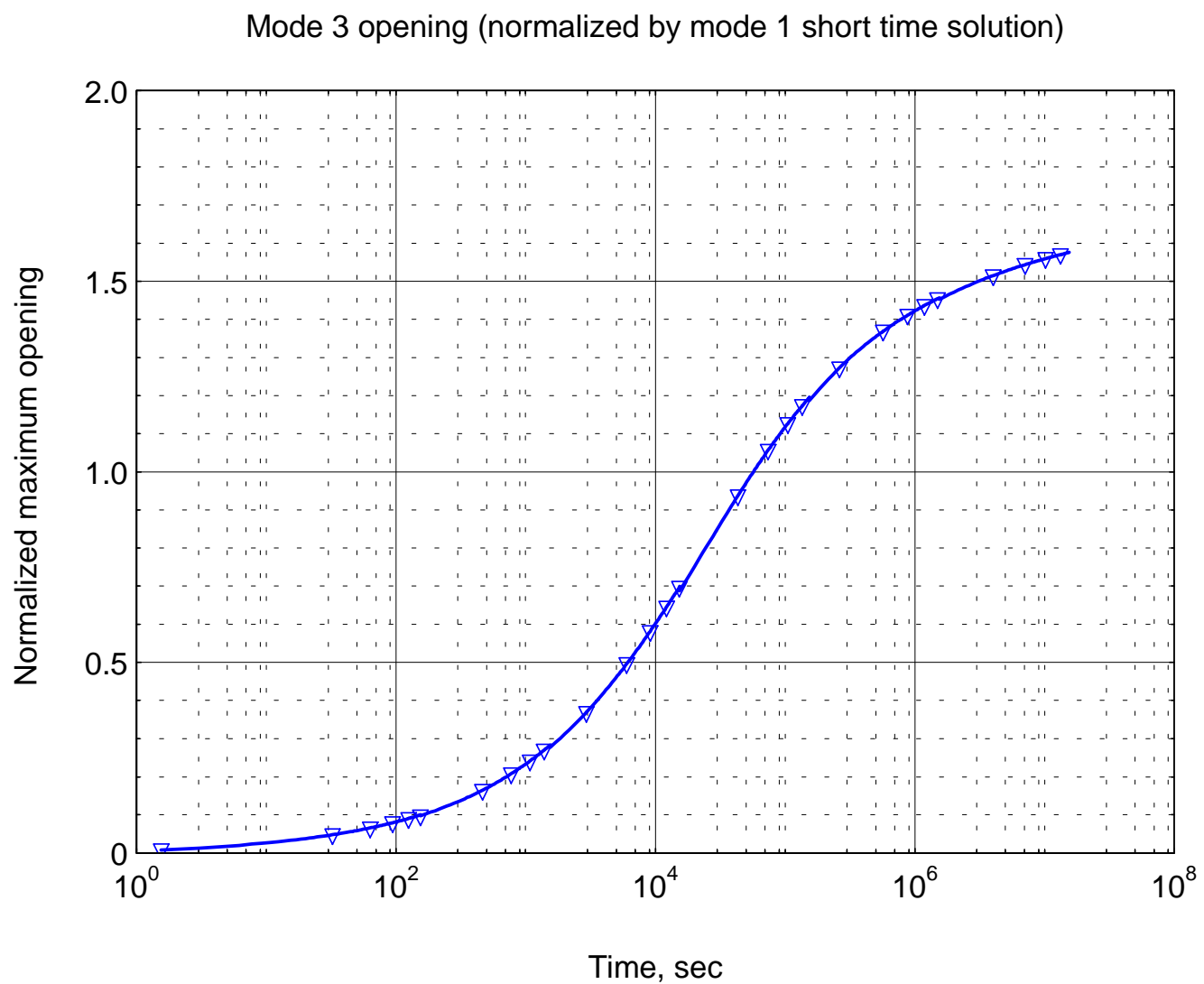

Figure 26: Time evolution of normalized (with respect to Mode 1 value at $t^{0+}$ ) crack width.

The fracture opening approaches the asymptotic value shown in red which is obtained by applying the thermal stresses that are generated by cooling, to the surface of a Griffith crack. For a two dimensional geometry (plane strain) it can be shown analytically that the stresses induced on the fracture faces (at steady-state) are given by:

$$
\sigma_{x x}=\sigma_{y y}=\frac{\beta_{s} E \Delta T}{3(1-\nu)}
$$

By comparing the fracture opening profiles as a function of time for various modes, it can be observed that in many practical situations in geothermal reservoir development, the contributions of Modes 1 and 2 to fracture propagation during stimulation can become negligible in comparison to cooling. This is evident from Figure 26 that illustrates the Mode 3 crack width normalized with respect to the instantaneous value of crack opening due to Mode 1.

However, these results indicate that the contribution of Mode 3 or thermal stresses, is significant in circulation operations that are carried out over a long period of time such that the thermal front has had sufficient time to develop. It should be noted that the impact of Mode 2 can significantly increase if one takes 


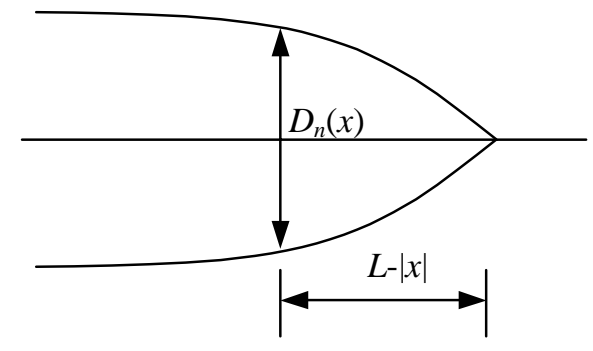

Figure 27: Location of the point where DD's are calculated for SIF determination.

into consideration the increase in rock permeability due to thermal cracking. The contribution of various mechanisms can be further illustrated by plotting the temporal evolution of SIF's for each mode.

Stress intensity factor The opening and shear mode stress intensity factors (SIFs) are used to define the magnitude of the singular stress and displacement fields (local stresses and displacements near the crack tip). The classical elastic relationship between the crack opening displacement and the SIF is given by:

$$
K_{1}=\frac{\sqrt{\pi} G}{\sqrt{8}(1-\nu)} \lim _{|x| \rightarrow L} \frac{D_{n}(x)}{\sqrt{L-|x|}}
$$

Where $G$ is shear modulus, $D_{n}$ is crack opening at a point $(x, 0)$ near the crack tip, as shown in Figure 27 , and $(L-|x|)$ is the distance from this point to the tip. As mentioned previously, the calculated crack opening $D_{n}(x)$ is least accurate near the tip. Thus, in this report the displacements are calculated at the point $(0.872,0)$, where $(L-|x|) / L=12.8 \%$. The value of $D_{n}(x)$ at this point has an acceptable error of about $5 \%$. It should be mentioned that an accurate estimate of the stress intensity factor requires implementation of a square-root tip element [130] but is not pursued here at this time. Note that $\left(\hat{D}_{n}\right)_{\max }=\frac{D_{n}(0, t) G}{\sigma_{n} L}$ and that $K_{1}$ has a unit $\left(\mathrm{MPa} \cdot \mathrm{m}^{1 / 2}\right)$. The normalized SIF is defined by $\hat{K}_{1}=\frac{K_{1}}{\sigma_{n} \sqrt{L}}$. In the plots below, the short-term value (initial value) of the SIF is calculated using [123]:

$$
\hat{K}_{1}\left(0^{+}\right)=\frac{\left(1-\nu_{u}\right)}{(1-\nu)} \sqrt{\pi L}
$$

There is no induced displacement on the crack surface by Mode 2 loading at time $t=0^{+}$, so the initial value of the SIF is zero. The final value of the normalized SIF is $(-0.2626)$, obtained from [123]:

$$
\stackrel{\wedge}{K}_{1}(\infty)=-\eta \sqrt{\pi L}
$$

The calculated SIF is normalized as $\hat{K}_{1}=\frac{K_{1}}{P \sqrt{L}}$. Note that the negative SIF for Mode 2 loading should be interpreted as a reduction of the (positive) Mode 1 loading stress intensity factor.

A comparison of the stress intensity plots further illustrates the significance of thermal stresses in fracture propagation at large times that are typically encountered in injection and circulation operations. In fact, the thermal stresses can exceed the contributions of the hydraulic pressure applied to the crack surfaces. 


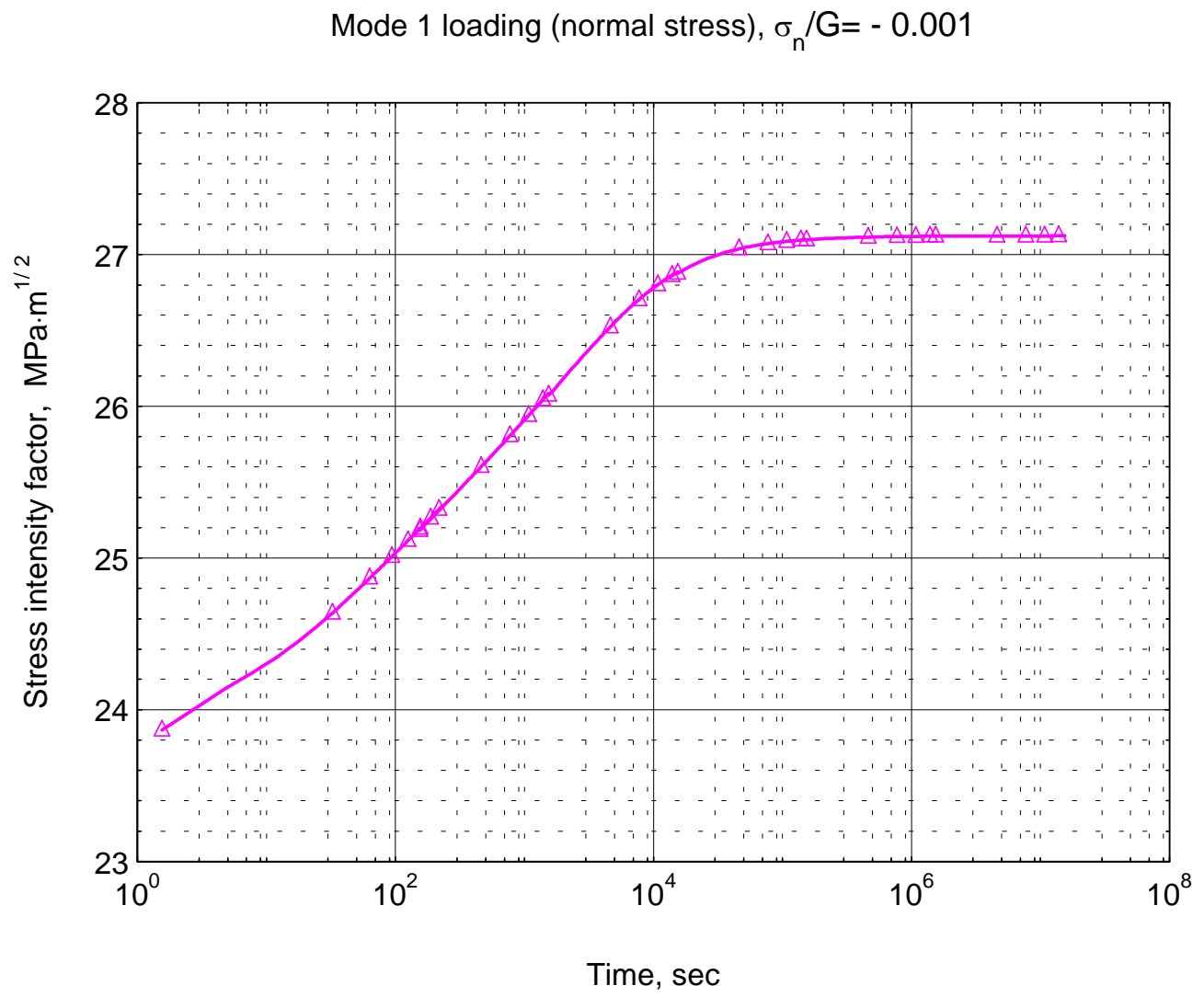

Figure 28: Magnitude of SIF for mode 1 loading. 


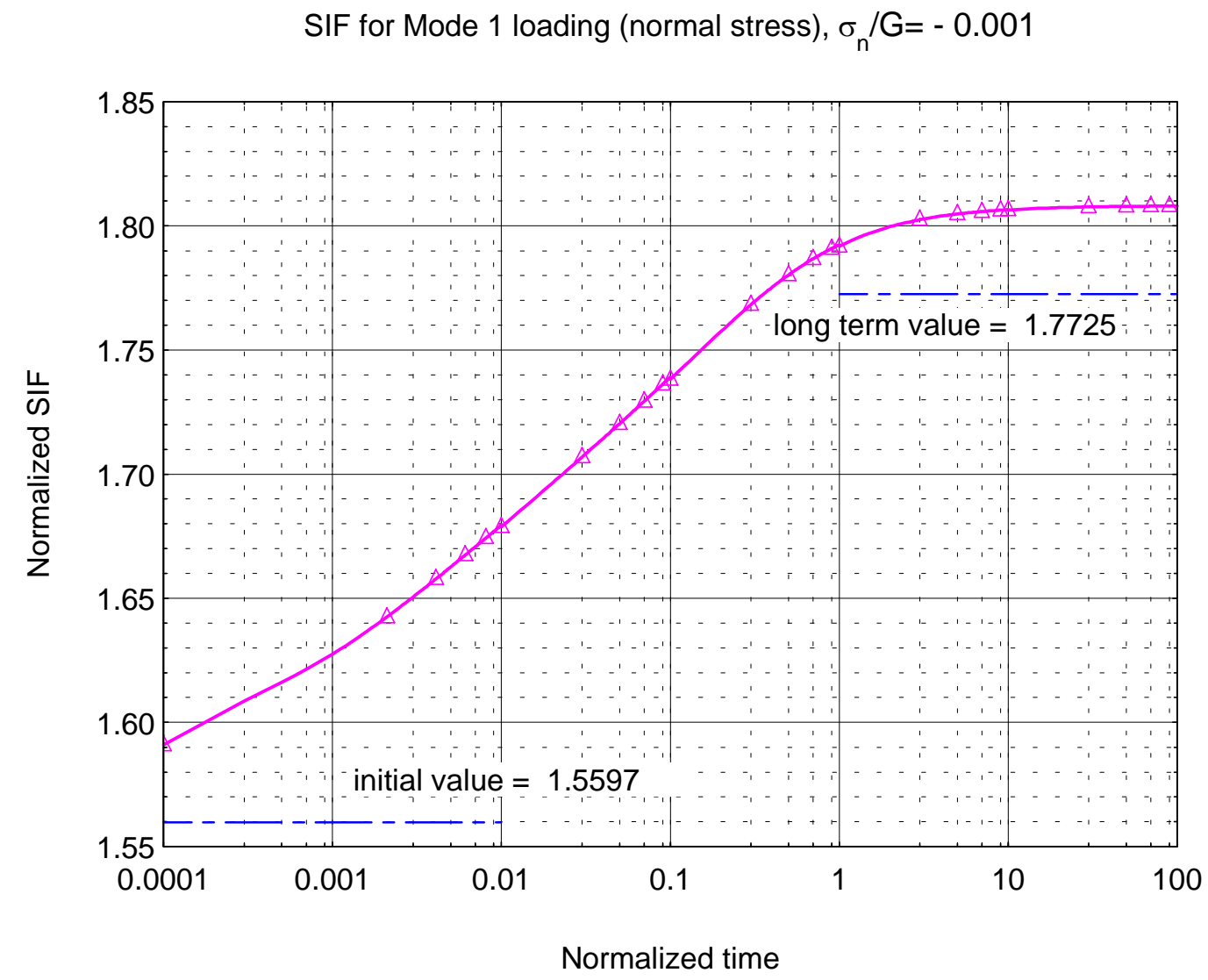

Figure 29: Normalized SIF due to stress loading. 
SIF for Mode 2 loading (pore pressure), $P / G=0.001$

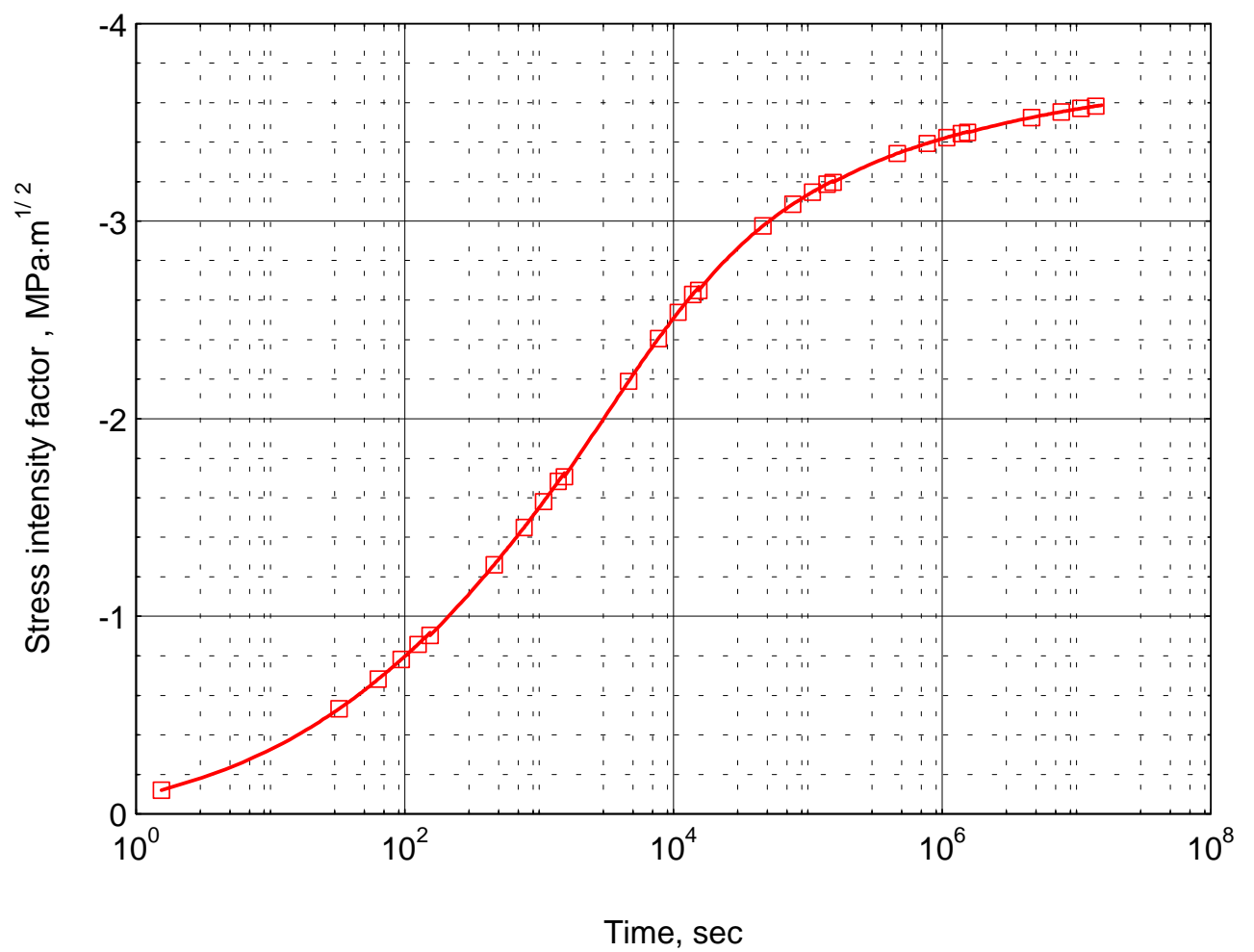

Figure 30: SIF due to pore pressure loading. 
SIF for Mode 2 loading (pore pressure), $P / G=0.001$

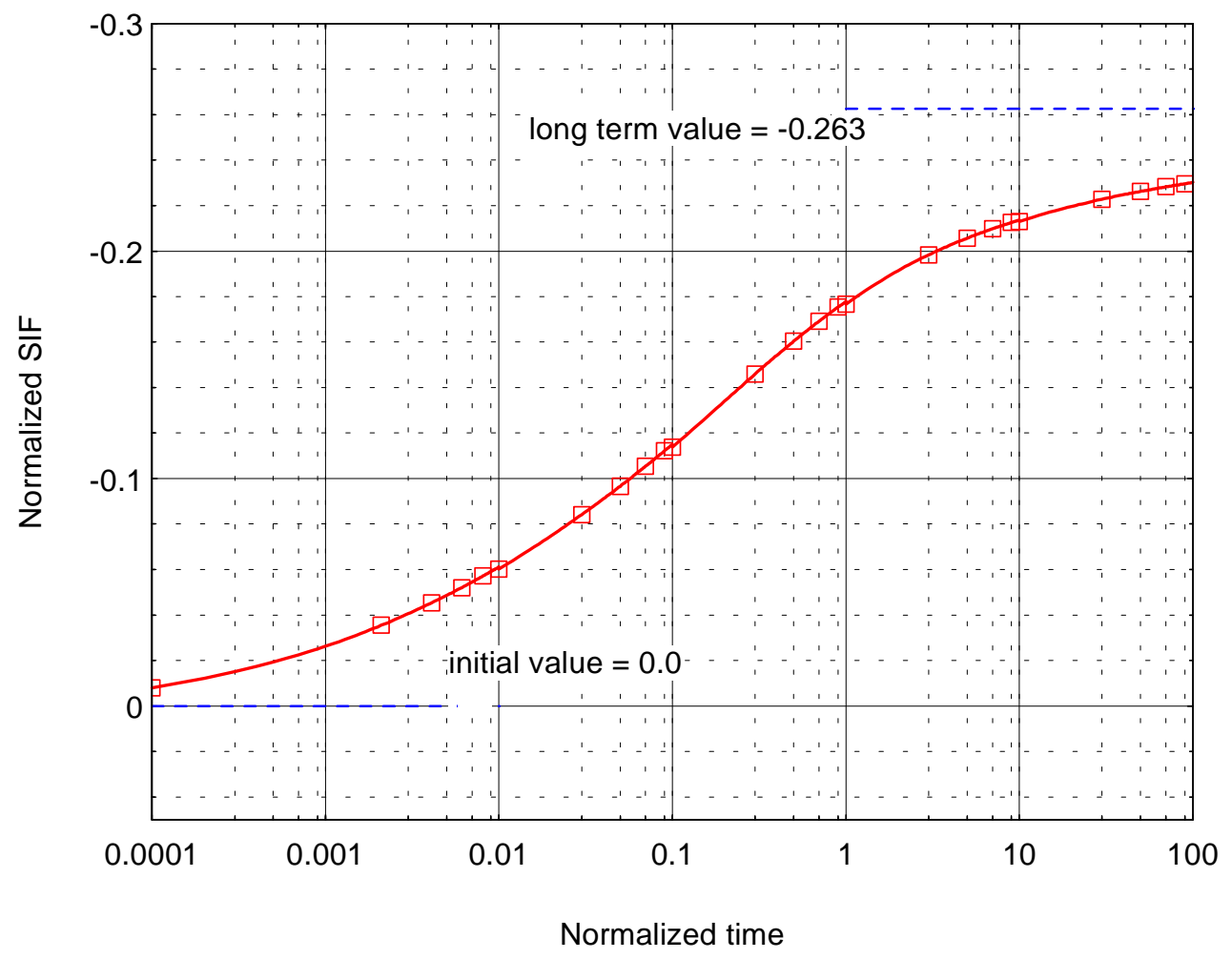

Figure 31: Normalized SIF for pore pressure loading. 


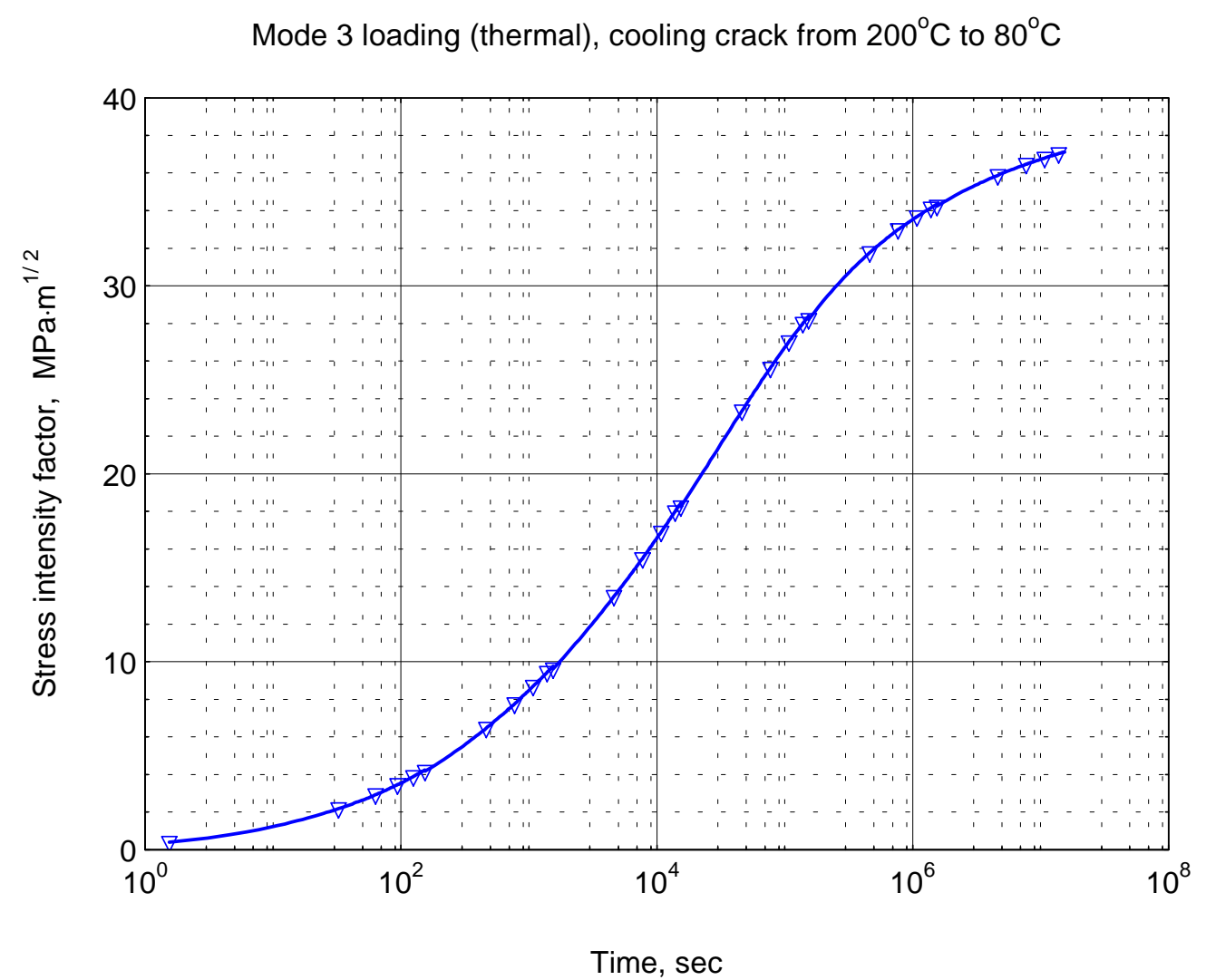

Figure 32: Temporal evolution of stress intensity factor due to cooling. 


\subsection{Modeling Fracture Propagation}

A specific type of boundary element technique called the displacement discontinuity method (DDM) is used in this part of the study to develop a two-dimensional boundary element code for modeling hydraulic fracturing in a geothermal environment. The DDM is particularly well-suited to hydraulic fracturing problems because the unknowns of the method represent the actual fracture opening and ride. A special variant of the DDM technique, the complex variable displacement discontinuity formulation [72]-[92] is used in the fracture propagation model. In this approach to DDM the displacement discontinuities are defined from the numerical solution of a complex hypersingular integral equation written for the fracture of a given configuration and loading. The main advantages of the complex variable approach are:

1. The ability to consider fractures in piecewise homogeneous media, i.e., to take into account inclusions, openings and notches (this is important for studying fracture interactions)

2. The use of a circular arc (straight segment as a degenerated case) to model the crack path increment at each propagation stage. Because the tangents at the contact between neighboring arcs of circles are kept the same, one can model smooth crack growth.

3. The use of high order approximating polynomials with square-root asymptotics for the unknown functions (DD)

4. The use of a circular arc (straight segment) for segments of the crack path that allows one to avoid the numerical integration under the solution of the hypersingular integral equation. This leads to high accuracy in the calculation of the stress intensity factors

Details of this novel technique, its numerical procedures, and illustrative examples are described next.

\subsubsection{Complex variable integral equation method}

The prediction of crack propagation is an important area of study in fracture mechanics. Considerable research has been conducted on experimental, analytical, and numerical modeling of crack growth by previous researchers (see [64]-[65] and references therein). The analytic and numerical methods to model crack propagation may be divided in two main classes [63]:

- the differential (stepwise) methods based on a local criterion of fracture propagation; and

- the integral (global) methods based on a global criterion.

The stepwise method is used in most works employing numerical simulation of fracture propagation. The crack path is considered as a sequence of small steps. At each step of propagation, the crack is extended from the previous tip by a length increment $\delta \ell$ in some direction which is found by choosing an appropriate propagation criterion. Extensive discussions on various criteria for fracture propagation may be found elsewhere (see Section 1 and [63], [64]); however, the most common criterion used is that of maximum tensile stress. The stress intensity factors (SIFs) involved in this criterion are calculated using a boundary element (BEM) or the finite element (FEM) method. Therefore, both a method and a criterion of fracture propagation are necessary to model fracture growth. This approach to modeling fracture propagation leads to a crack path that resembles a jagged line, contrary to the expectation of a smooth crack path (except, 
perhaps, at the point of initiation). The only stepwise method that yields a smooth path is suggested in [62], in which the maximum tensile stress criterion is used and the crack path at each stage of propagation is approximated by a cubic parabola. The complex singular integral equation is solved by means of a mechanical quadrature method to calculate the SIFs. However, the use of the maximum tensile stress criterion leads to a crack path in the form of a waving line (the deviation's angle $\theta$ will change its sign at the two neighboring stages). Moreover, use of the cubic parabola as the part of the crack path leads to formation of singular integral equations that must be numerically integrated to obtain a solution. This increases computational time and decreases the level of accuracy. In addition, the use of the singular equation in crack problems requires satisfaction of additional conditions, namely, that the displacement discontinuity (DD) be zero at the crack tips.

In this project, a new, simpler method [76] and [69] is used which not only provides for a smooth crack path but also alleviates the disadvantages mentioned above. This particular formulation will enable the user to numerically simulate the propagation of fluid-pressurized fractures in piece-wise homogeneous finite, infinite, and semi-infinite domains, taking into account thermally-induced stresses. The fracture propagation scheme is comprised of two procedures, or two criteria of crack propagation. First, the propagation direction is determined by assuming that the fracture grows in the direction perpendicular to the maximum circumferential tensile stress. Then, one exploits the fact that the mode II stress intensity factor $K_{I I}$ is equal to zero at the current crack tip. The crack is extended from the previous tip once the condition for crack propagation $K_{I}=K_{I c}$ is satisfied (where $K_{I}$ is the mode I stress intensity factor, and $K_{I c}$, the toughness). The stress intensity factors $K_{I}$ and $K_{I I}$ are calculated at each step of propagation via the known displacement discontinuities (DDs) at the fracture surfaces. The DDs are found using a boundary element method based on the analytic solution to a complex hypersingular integral equation (CHSIE).

The crack path at each stage is approximated by a circular arc and contrary to other approaches that use approximate formulae (see e.g. [64]), the SIFs are calculated using analytical formulae that are valid for any curvilinear crack. The use of a hypersingular equation does not lead to any additional conditions and the use of the circular arc (or straight segment as a limiting case of the circular arc) allows one to avoid numerical integration.

The method used in here is universal so that the crack can be situated in a piecewise homogeneous plane. This means that crack propagation in a piecewise homogeneous half-plane (the corresponding CHSIE was obtained in [93]), bonded half-planes, etc. can be handled. Furthermore, growth and interaction of multiple cracks can also be considered.

Details of the numerical technique are reported in Appendix 1. However, a few key examples are presented next in order to illustrate the program capabilities and its high level of robustness. These examples also serve to illustrate that the program is very accurate and that the complex hypersingular integral equation (196) and formulae (195) in Appendix A provide reliable expressions for calculation of the SIFs.

Crack path under uniaxial stress Consider the problem of predicting the path of a straight crack located on the interval $|x| \leq a$ of $x$-axis in an infinite plane under uniaxial stress, $p$. The angle between the direction of loading and the $O x$ axis is given by $\gamma$. The crack path was calculated using the new method and is plotted in Figure 33 for the different values of $\gamma$. The results are in a good agreement with the numerical solutions reported in [58] and [62]. 


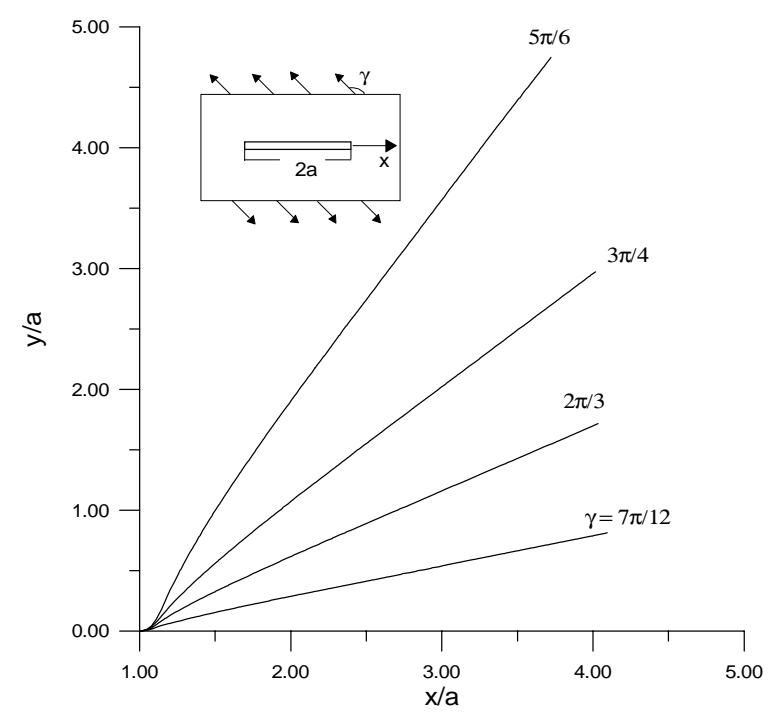

Figure 33: Crack path under uniaxial stress.

Crack path under biaxial stress The crack is again situated on the interval $|x| \leq a$ of $x$-axis, but the stresses at infinity are: $\sigma_{x x}=q, \sigma_{y y}=p$, and $\sigma_{x y}=0$. Due to the symmetry the crack will always grow straight. This is not the case if it is assumed that during the first propagation step the crack grows at an angle $\theta=5^{\circ}$ to the direction of $O x$ (this assumption is made in [62]). The crack paths for different values of $q / p$ are plotted in Figure 34. The results are consistent with the numerical, analytical, and experimental results reported in [62], [64], and [56], respectively.

Crack propagation near a circular inclusion in a biaxial stress field Suppose that the crack is situated on the interval $|x| \leq a$ of $x$-axis and that the stresses at infinity are given as: $\sigma_{x x}=q, \sigma_{y y}=p$, $\sigma_{x y}=0$. Further, suppose that there is a perfectly bonded circular inclusion having a radius $R$ with its center at the point $O\left(x_{0}, y_{0}\right)$. The problem is solved numerically for the case when $x_{0}=3 a, y_{0}=2 a$. In addition, different values of radius $(R / a=0.5 ; 0.75 ; 1.0)$ and shear modulus $(\mu 2 / \mu 1=0.3 ; 1.0 ; 3.0)$ are considered. In the case when $\mu 2 / \mu 1=1.0$, it is assumed once again that the crack will initially propagate at an angle of $\theta=5^{\circ}$ to the $O x$ direction. Figures 35-37 illustrate the crack paths for $q / p=2$. As expected, the presence of the circular inclusion with different elastic properties has a significant influence on the crack path. This influence increases with an increase in the size of the inclusion.

To summarize, a procedure for simulating two-dimensional crack propagation is used. It is a stepwise method based on a local fracture propagation criterion. These expressions involve displacement discontinuities that result from the numerical solution of a complex hypersingular integral equation for a piecewise homogeneous plane with curvilinear cracks. The crack path is approximated smoothly using circular arcs. A numerical algorithm based on this method yields accurate solutions for complex fracture propagation problems. 


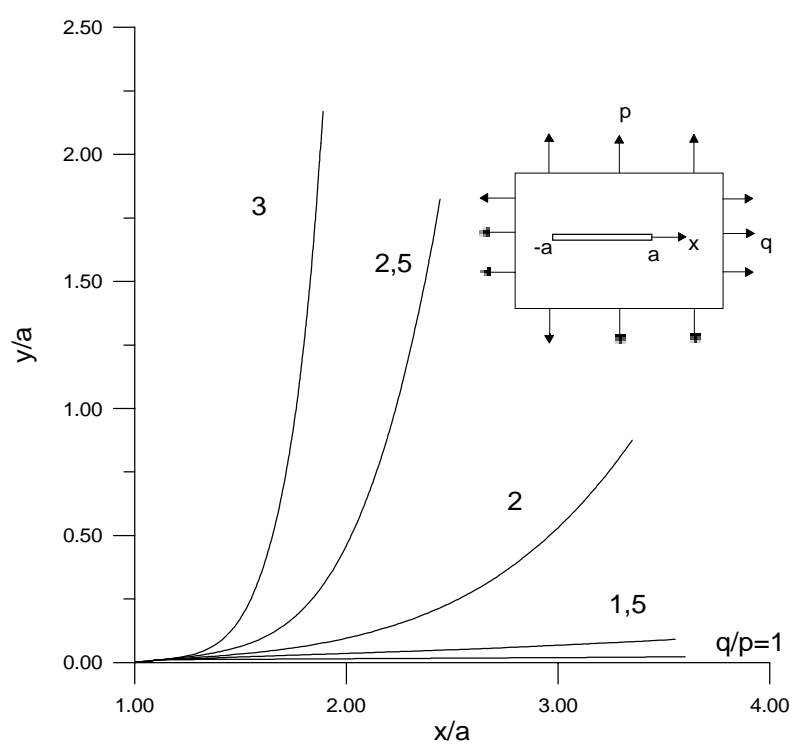

Figure 34: Crack path under biaxial stress.

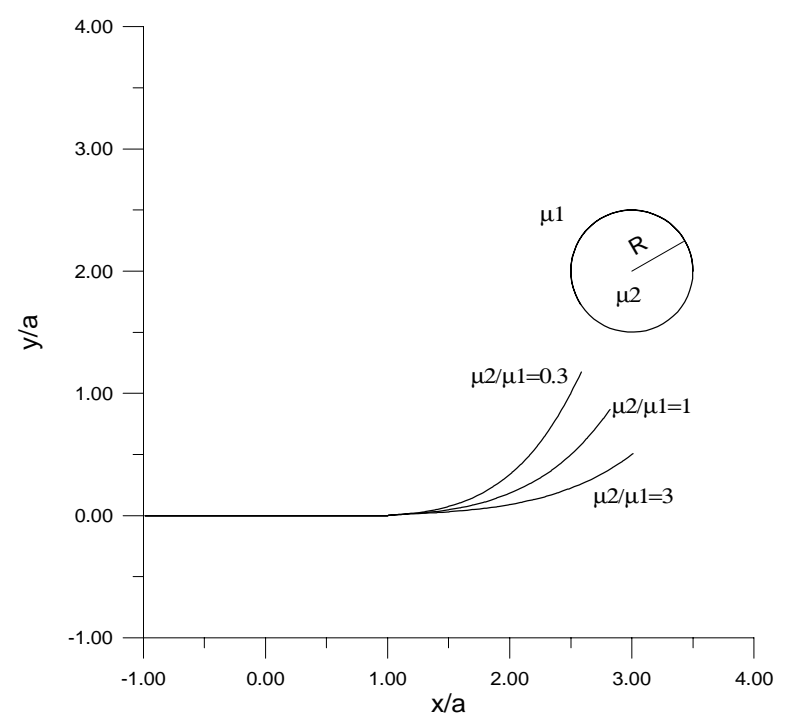

Figure 35: Crack growth near a circular inclusion under biaxial stress $(R / a=0.5 ; q / p=2)$ 


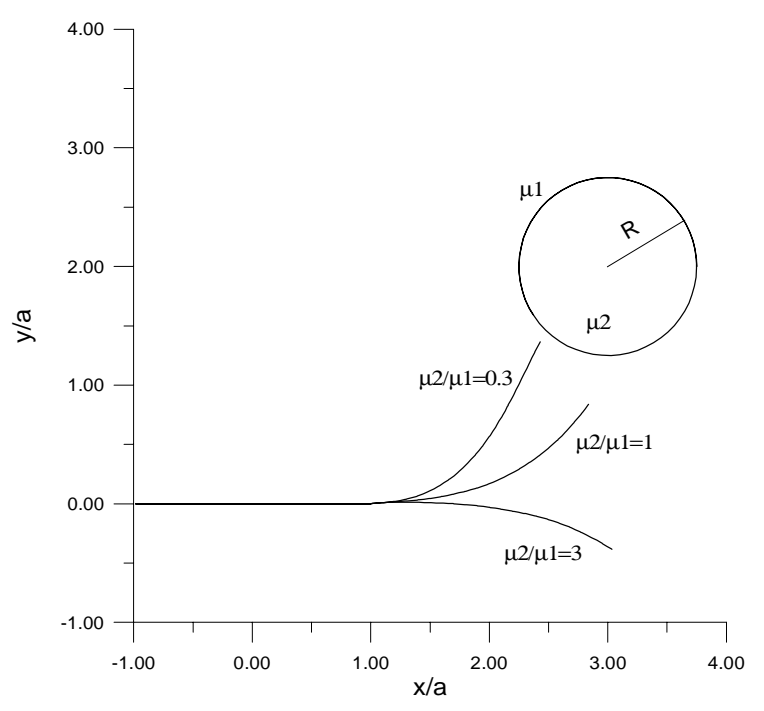

Figure 36: Crack growth under biaxial stresses near the circular inclusion $(R / a=0.75 ; q / p=2)$

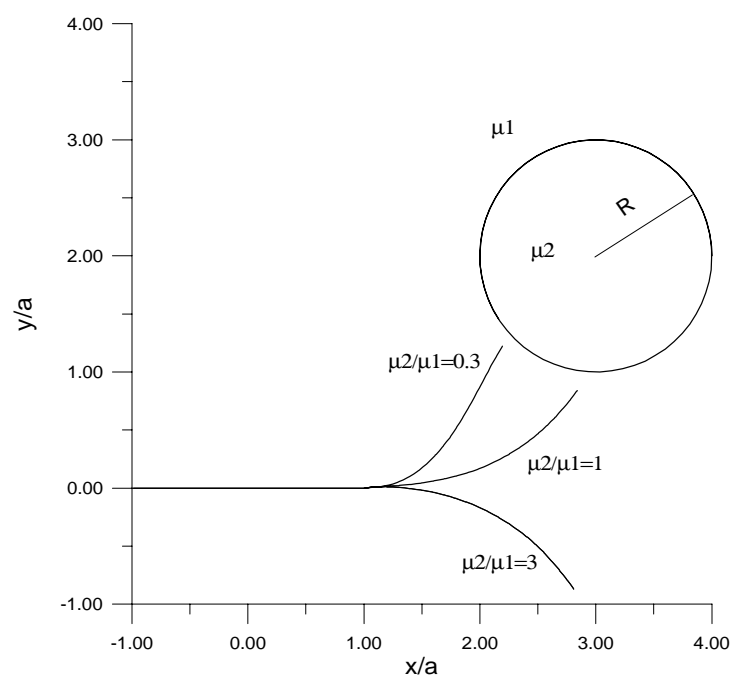

Figure 37: Crack growth near a circular inclusion in a biaxial stress field $(R / a=1.0 ; q / p=2)$. 


\section{Examples \& Applications}

\subsection{Fracture Propagation from a Wellbore: An Isothermal Fracture Mechanics Analysis}

The problem of wellbore failure and fracture initiation was studied using the classical approach in Section 3 , while considering the role of transient thermal and poromechanical effects. However, as indicated previously, a fracture mechanics approach can capture certain phenomena that the classical approach cannot explain. Thus, a numerical scheme is useful to study fracture initiation and propagation. The scheme is isothermal and neglects pore pressure effects at this time. The fracture propagation pressure is determined through application of a fracture propagation criterion within the framework of a step-wise algorithm [76]. The stress intensity factors $K_{1}$ and $K_{2}$ used at each propagation step are calculated using known displacement discontinuities (DDs) at the fracture surfaces. These DDs are defined from the numerical solution of a complex hypersingular integral equation (CHSIE) written for the fracture configuration and loading at each step. The corresponding CHSIE for a plane with a circular opening and a system of arbitrary curvilinear cracks has been obtained in [67] and the basic equations as well as the fundamental aspects of the numerical procedures are reported in Appendix 1.

As an example of the application of the model to fracture initiation, we use the problem of calculating the fracture initiation pressure for a wellbore in an infinite homogeneous elastic isotropic medium as studied by [88] and [76] (Figure 38). The wellbore has a radius $R$ and its center is located at the origin of the global Cartesian coordinate system with axes in the direction of in-situ stresses. The well is drilled in a biaxial compressive far-field stress $\sigma_{H} \geq \sigma_{h}$ (compression positive) with $\Pi=\sigma_{H} / \sigma_{h}$. As required by the fracture mechanics approach to initiation, it is assumed that the wellbore boundary contains a preexisting crack of length $\ell$ that is inclined at an angle $\alpha$ to the direction of maximum principal stress (38). The wellbore is subjected to an internal pressure $p$ which may be applied at a slow rate or a fast rate [65] [66]. These two pressurization schemes can be represented by two different pressure conditions at the crack boundary, namely, $(i)$ a traction-free crack, and $(i i)$ a pressurized crack with an internal pressure $p$. Due to pressurization of the wellbore, the preexisting crack will grow into a hydraulic fracture that will eventually orient itself in the direction of maximum in-situ stress. The results of the numerical solution $\left(p_{N}\right)$ for the case of $\ell / R=0.001, \Pi=2, K_{1 C}=0.25$ are shown in Table 1 . The fracture initiation pressure for the special case of a very small initial crack having a length $\ell<<R$ has been estimated analytically [63]. The propagation pressure in this case is determined by the SIF of an edge crack perpendicular to the surface of a traction-free half-space:

$$
\begin{cases}K_{1 C}=2.24 \sqrt{\pi \ell}(2 p-\sigma) & \text { fast rate } \\ K_{1 C}=1.12 \sqrt{\pi \ell}(2 p-\sigma) & \text { slow rate }\end{cases}
$$

where $\sigma$ is the stress away from the fracture. In the context of the present wellbore problem, illustrated in Figure 38, $\sigma$ is the hoop stress which can be obtained from Kirsch solution:

$$
\sigma=\sigma_{h}\left[3-\Pi+4(\Pi-1) \sin ^{2} \alpha\right]
$$

The analytical asymptotic solution $\left(p_{A}\right)$ is also shown in Table 1 . As can be observed, there is good agreement between numerically calculated values and the asymptotic solution for both pressurization rates. 

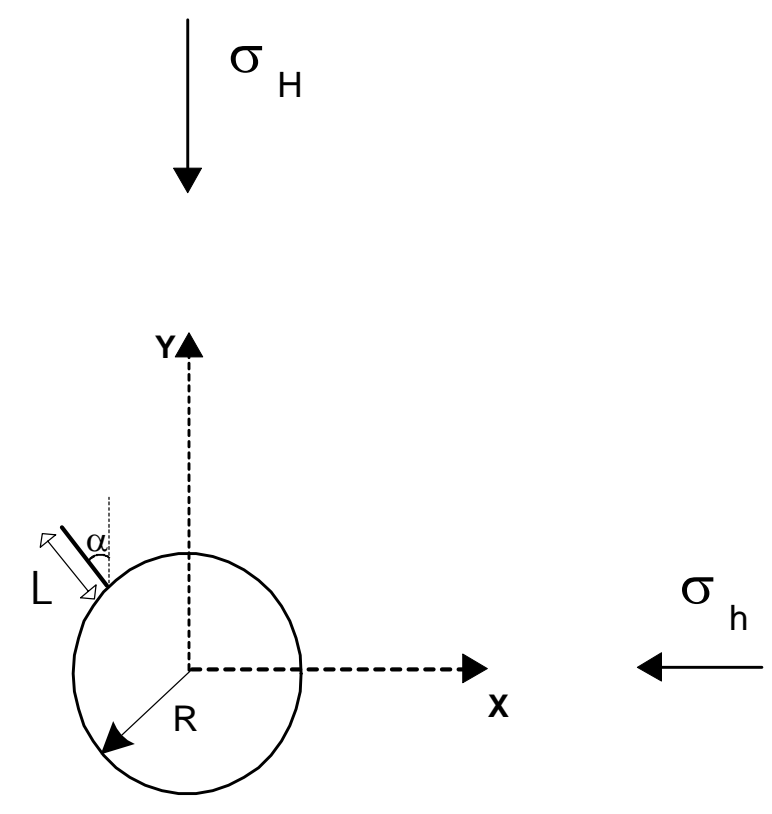

Figure 38: Problem geometry for studying fracture initiation and propagation from a wellbore.

Table 1. Fracture initiation pressure

\begin{tabular}{|l|l|l|l|l|l|l|}
\hline & & Slow & rate & & Fast & rate \\
\hline$\alpha$ & & $p_{A}$ & $p_{n}$ & & $p_{A}$ & $p_{n}$ \\
\hline 81 & & 4.4423 & 4.4376 & & 8.8845 & 8.8807 \\
\hline 63 & & 4.0790 & 4.0743 & & 8.1580 & 8.1536 \\
\hline 45 & & 3.4912 & 3.4875 & & 6.9824 & 6.9792 \\
\hline 27 & & 2.9034 & 2.9002 & & 5.8064 & 5.8039 \\
\hline 0 & & 2.5402 & 2.5379 & & 5.0803 & 5.0788 \\
\hline
\end{tabular}

\subsubsection{Influence of various parameters on propagation}

Fracture propagation studies suggest that the fracture path is a function of the difference between the in-situ stresses, $\sigma_{H}-\sigma_{h}$ (this conclusion seems obvious as fractures propagate along a linear path when the stress field is hydrostatic), the initial angle of crack, $\alpha$, and its initial length $\lambda=\ell / R$. In order to determine the nature of these dependencies and to elucidate their role, the problem of fracture growth from a wellbore is studied. For the sake of generality of results, the stress difference is normalized by $K_{1 C} / \sqrt{R}$ and the following parameter controlling the fracture path is introduced: $\beta=\frac{\sigma_{H}-\sigma_{h}}{\frac{K_{1} C}{\sqrt{R}}}=\frac{\Pi-1}{K}$ where $\widetilde{K}=K_{1 C} / \sigma_{h} \sqrt{R}$.

Slow pressurization rate Several fracture paths for different values of $\beta$ are plotted in 39. For a hydrostatic far-field stress $(\beta=0)$ the fracture propagates in the radial direction along the preexisting fracture. Any non-zero $\beta$ results in fracture turning and eventually running along the direction of maximum stress. As $\beta$ increases, the fracture undergoes a sharper turn and reaches the favorable path faster. Based on a series of computer runs, it can be concluded that the propagation paths of similarly oriented initial cracks coincide (within 5 digits) as long as the combinations of parameters involved lead to the same value of $\beta$ 


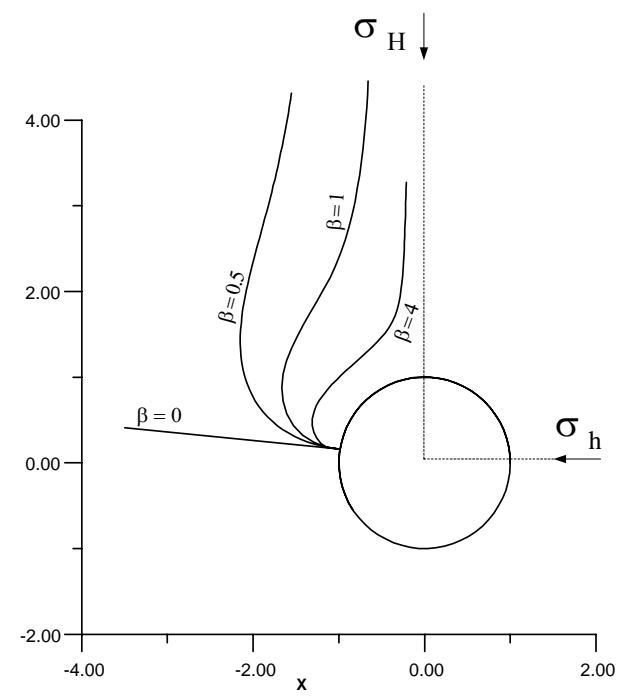

Figure 39: Dependence of the fracture path on dimensionless parameter $\beta$.

$[76]$.

Variation of propagation pressure along various paths is shown in 40. Although the path of the fracture is independent of the mean stress, the propagation pressure does dependent on it. The corresponding pressure for every path attains a minimum at a point corresponding to the inflection point of the path. When the inflection point does not exist (for low $\beta$ ), the pressure curve has no minimum.

41 illustrates the magnitudes of the minimum propagation pressure plotted against the stress ratio $\Pi$ for various fracture paths corresponding to a range of $\beta$ and initiating at the same point. Note that a minimum propagation pressure exists for all values of $\beta$ considered in the figure.

Dependence of fracture path and propagation pressure on inclination angle As can be observed in 42 , the orientation of the initial fracture has a strong effect on the fracture path . The fracture tends to return to what would be the path of the "favorably oriented fracture". The propagation pressures exhibit the already mentioned minimum at an inflection point of the path (43). The propagation pressure drops to a rather low value irrespective of the orientation of the preexisting fracture. In general, higher values of the stress ratio lead to lower minimum values of the propagation pressure.

Dependence of fracture path on propagation increment Computations show that the fracture path is practically independent of the fracture increment length. This has been checked for increment lengths as small as $s=1 / 40$ of the wellbore circumference.

Dependence of the fracture path and of initiation pressure on the preexisting crack length For a fixed value of $\beta$, the fracture path is only weakly dependent on the initial fracture length. The fracture tends to return to a straight path as illustrated in 44 . However, the initiation pressure is strongly dependent 


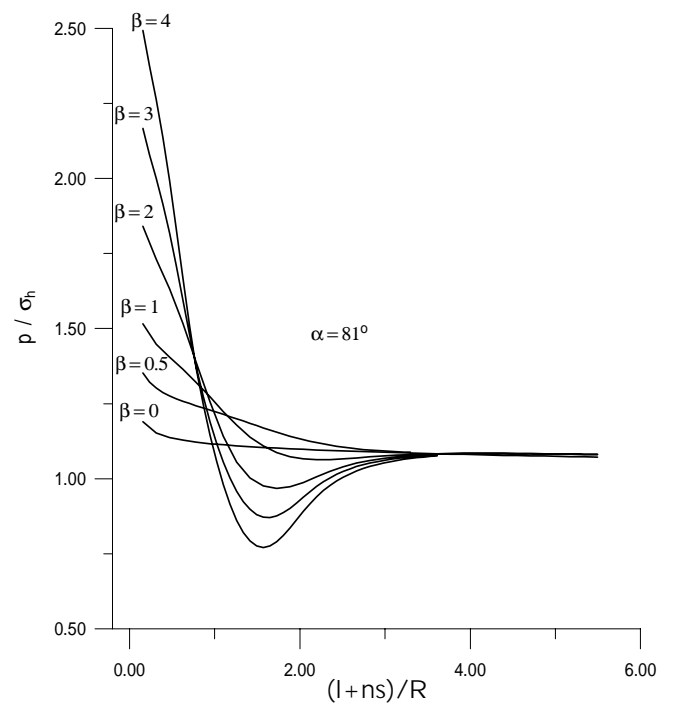

Figure 40: Variation of propagation pressure along the crack path ( $n$ is number of the propagation step, $\ell$ is the initial fracture length).

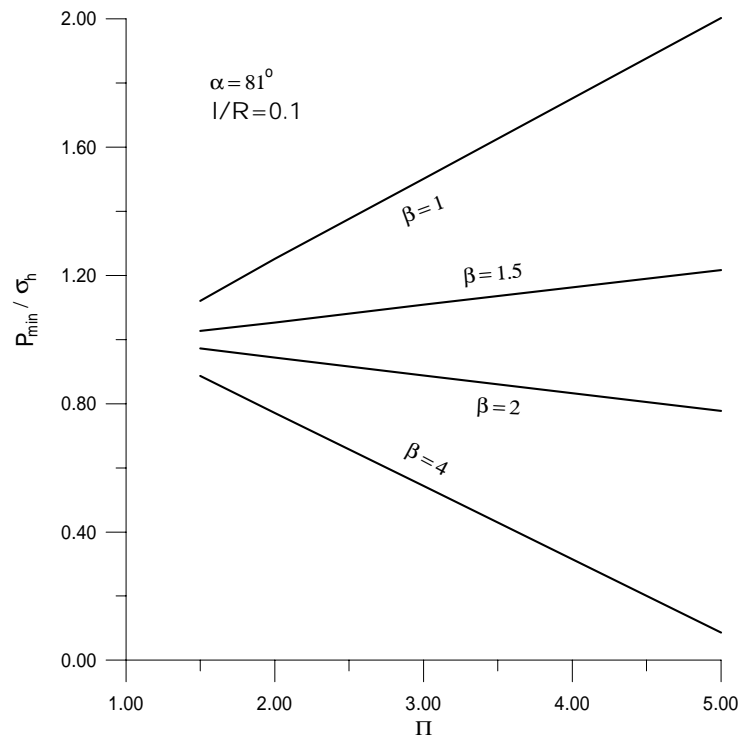

Figure 41: Dependence of the minimum of propagation pressure on $\Pi$. 
on the initial fracture length for all initial orientations of the fracture. This dependency is illustrated in 45 for $\beta=4$.

Fast pressurization rate When calculations are carried out without fluid pressure penetrating the crack, fractures generally follow the path along the initial radial direction with little curvature (see 46). Pressures required to propagate the fracture are high and increase rapidly with the fracture length, 47 . This type of behavior has been observed in laboratory experiments [56].

The above parametric study of hydraulic fracturing process from the point of view of fracture mechanics for conditions of slow and fast pressurization rates suggests that a dimensionless parameter, defined in terms of the far-field stress deviator, fracture toughness, and wellbore radius, influences the fracture path. It has been observed that the fracture path is determined by the far-field stress deviator and the fracture toughness. The path is very sensitive to these parameters when both are small.

\subsubsection{Hydraulic fracture propagation near a fault}

In this section we study fracture propagation near a natural discontinuity. This is an important problem in modeling hydraulic fracture propagation in a naturally fractured reservoir. The numerical model described above cannot be used to treat this problem, and a different numerical scheme is necessary to address two non-linear effects namely, changes in geometry caused by the growing cracks; and irreversible deformations on contact between surfaces. These effect are simultaneously accounted for by using a method discussed in [101]. This method has been implemented in CVBEM of [103] and is used herein.

Problem geometry and boundary conditions We suppose that all of the forces applied to the boundary of the domain under consideration are known; and distinguish between external forces (weight or tectonic) and fluid pressure that acts on the wellbore walls and the fracture surfaces. As before, there are two modes (regimes) of pressurization, fast and slow. In the first case pressure acts only to the wellbore walls. The second one corresponds to the pressurization of both the wellbore and the fracture surfaces. These limiting cases are simplifications of the real situation. A rigorous formulation of the problem would address the initially unknown coordinates of the point where the type of boundary conditions changes and its determination as a part of the solution. Of course, one does not know a priori where the points of boundary condition alteration (i.e., coordinates of the front of the fluid penetration) will be along the fracture.

The goals of our studies allow us to exploit a simplified approach, similar to those in previous section and in [76]. We consider two extreme cases: (1) the total length of the crack is under fluid pressure; (2) the total length is free from pressure. There are reasons to predict that the real trajectory will be between these two trajectories corresponding to the mentioned limiting cases. We can consider different loading paths, meaning that the in situ stress components or fluid pressure may depend on time, space, or other parameters. The computational procedures allow for loads to evolve in an incremental manner.

Fracture model Consider a crack emanating from a wellbore in the vicinity of a fault (Figure 48). The radius of the wellbore is $R$; the crack has an initial length of $\ell$ and makes an angle of $\alpha$ with the y-axis. The distance from the center of the wellbore to the fault is $d$ and the angle of the fault with the y-axis is $\phi$. 


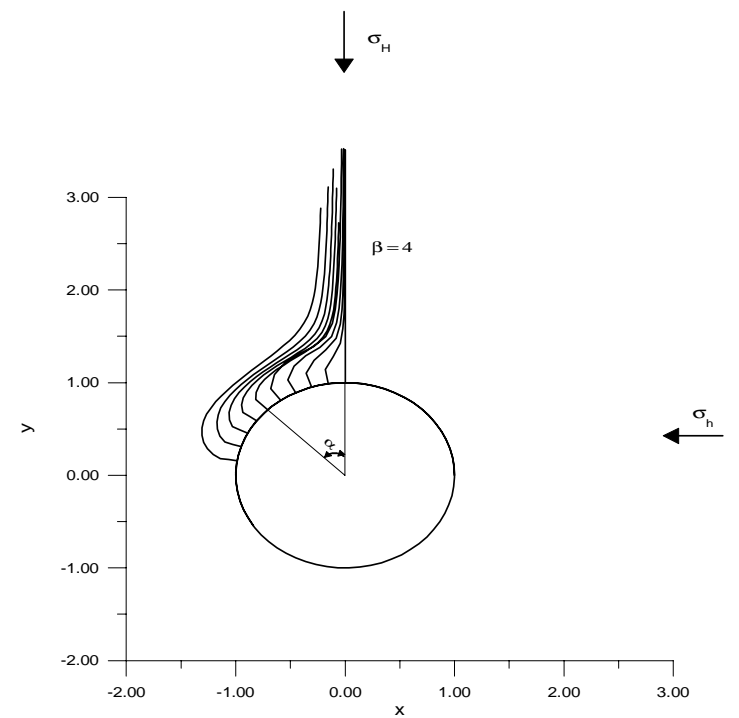

Figure 42: Dependence of the fracture path on the inclination angle $\alpha$ (slow rate).

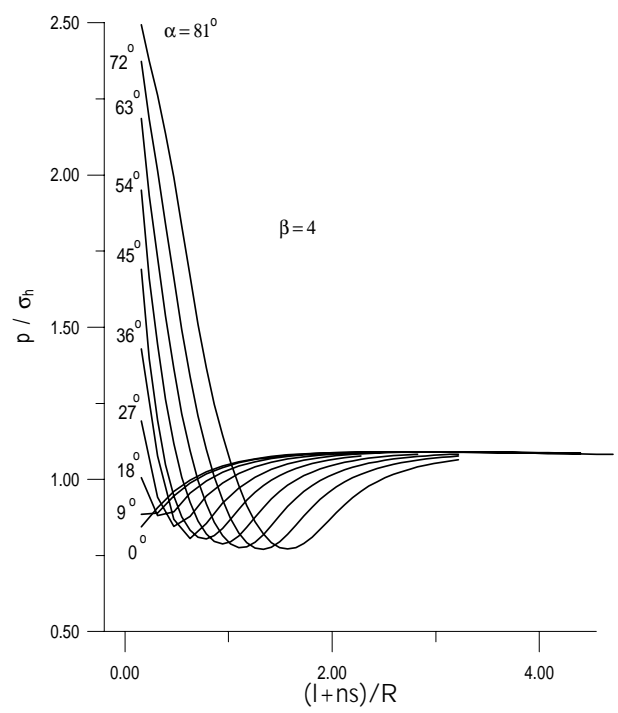

Figure 43: Variation of propagation pressure along the paths (slow rate). 


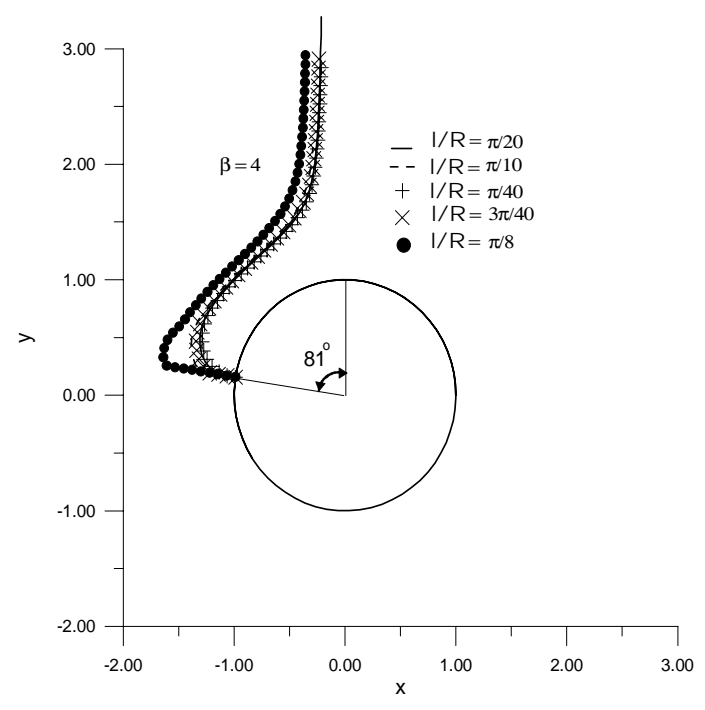

Figure 44: Dependence of the fracture path on preexisting crack length.

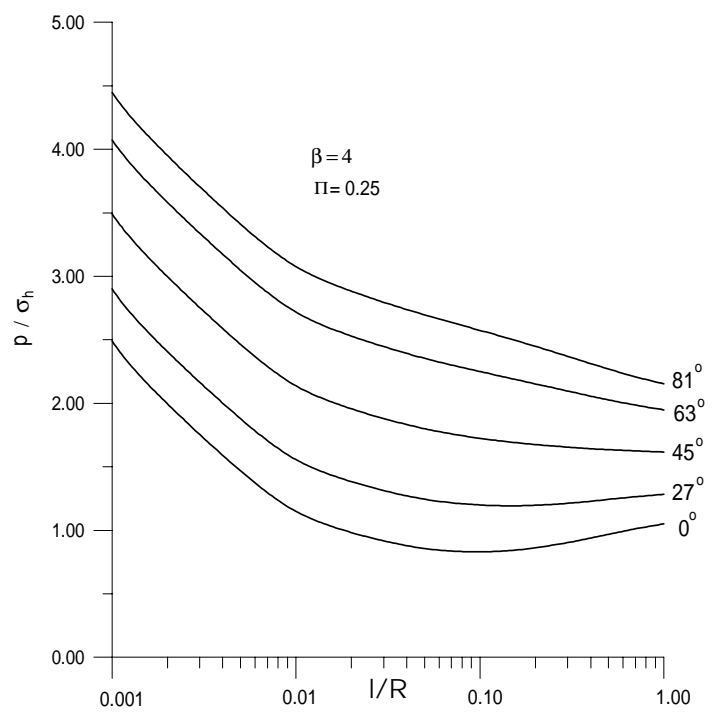

Figure 45: Dependence of the initiation pressure on the initial crack length, $\ell$. 


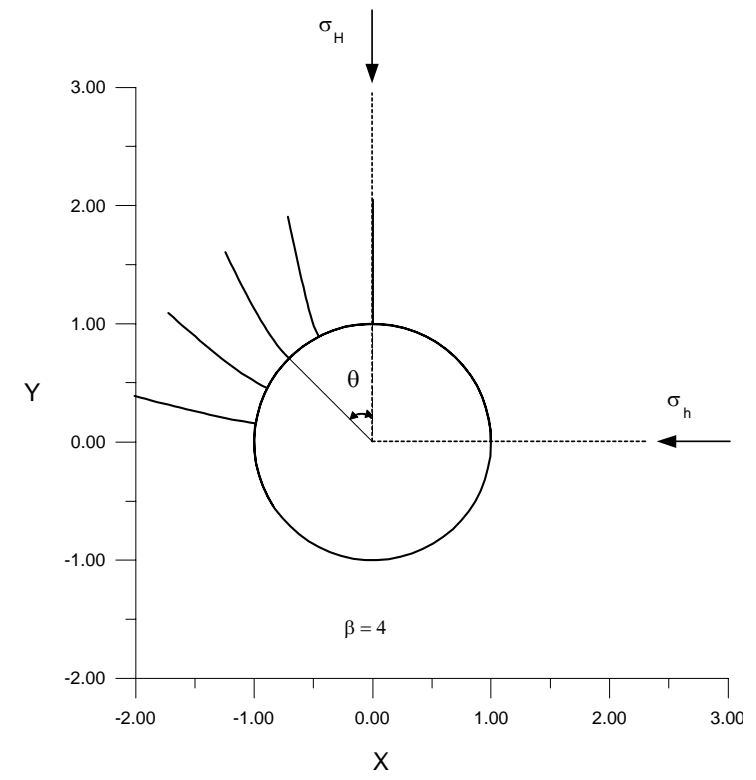

Figure 46: Dependence of the fracture path on the inclination angle $\alpha$ (fast rate).

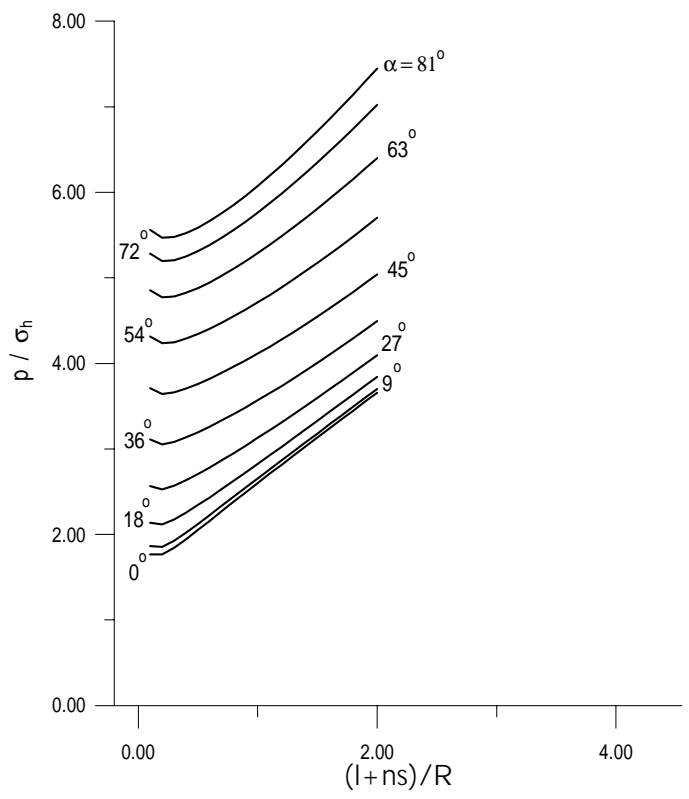

Figure 47: Variation of the propagation pressure along the paths (fast rate). 


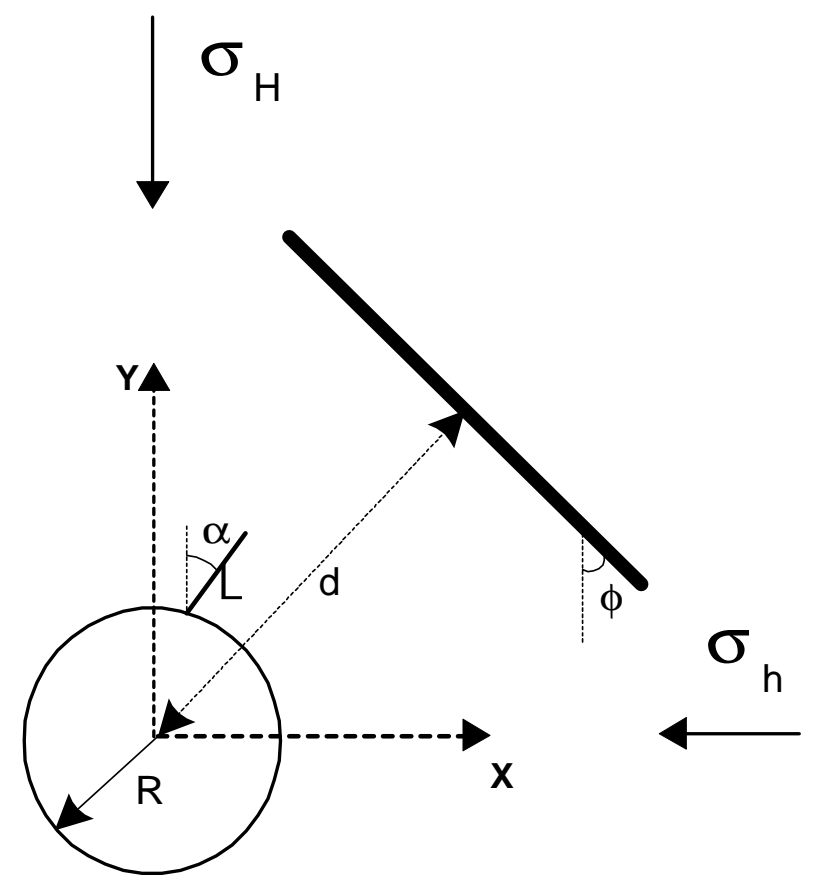

Figure 48: Problem geometry for fracture propagation from a wellbore in the vicinity of a fault.

It is further assumed that the initial crack is small enough not to propagate if it and the wellbore are not internally pressurized $(p=0)$. Then, the pressure $p$ increases from zero up to the value sufficient for crack propagation in mode I in accordance with the criterion:

$$
K_{I}=K_{I C}
$$

The crack trajectory is defined by the condition that crack grows in the direction of maximum $K_{I}$. It is well known (see, e.g., [132]) that this direction is defined by the condition:

$$
K_{I I}=0
$$

where $K_{I I}$ is the shear SIF. As mentioned above, in contrast to [76], we do not presume that the internal pressure $p$ is maintained at the level strictly satisfying (68) along the entire trajectory (stable propagation scheme). In other words, we do not exclude unstable (with $K_{I}>K_{I C}$ ) crack propagation once the condition (68) is satisfied. Following [69], [131], [132], and [133], we assume that unstable crack propagation occurs under unchanged external forces. Firstly, the minimum pressure $p_{0}$, corresponding to the initiation of crack growth is calculated. If this pressure leads to an unstable process along the trajectory, we take $p_{0}$ as the driving pressure, $p_{m}$. In the opposite case, when $K_{I}$ decreases with crack propagation, we increase $p$ from its initial value up to the pressure $p_{m}$ sufficient to restore (68) at the point of the minimum $K_{I}$. Thus, while (68) must be satisfied at least at one point on the trajectory, the condition: 


$$
K_{I} \geq K_{I C}
$$

is imposed at other points.

In most of the examples that we consider, the fracture faces are loaded by a fluid pressure (this is the case of slow pressurization). For the case of fast pressurization we assume that the fracture is closed, (practically, this means that $\left|\Delta u_{n}\right| \ll\left|\Delta u_{t}\right|$ ); only the tip element is open, and the fracture faces are frictionless: $\left|\sigma_{n t}\right| \ll\left|\sigma_{n n}\right|$. We suppose that the length of the preexisting fault or joint and its inclination are known in advance.

One can use (68) for an actual crack configuration, i.e., the result of previous steps of propagation; to find the loads necessary to continue crack growth. If we have a simple loading path (e.g., one component of the load does change or all the components change proportionally to one another) then Equation (68) has a unique solution which determines the critical condition for actual configuration. As before, we calculate the Mode II stress intensity factor, $K_{I I}$, at each step of propagation for a set of possible trajectories for future growth. Both circular arc and straight increment are used in extending the crack from the previous configuration. The choice of the actual direction from the possible set is based on the criterion $K_{I I}(t)=0$, where $t$ is a new crack tip coordinate.

Using the CVH-BEM algorithm as described in [101], we automatically calculate both the crack path and the changes on the fault surfaces. The problem is reduced to finding the crack trajectory, displacement discontinuities, and the tractions on the fault surface give that:

- $\sigma_{H}$ and $\sigma_{h}$ are prescribed at infinity

- fracture propagation is governed by the conditions (68)-(70)

- the condition on the fault is specified

Our objective is to elucidate the influence of the wellbore and/or a natural discontinuity with various characteristics and their mutual interactions with a propagating crack. Also, we aim to evaluate the role of material inhomogeneity. We suggest (and can consider) that the growth of a preexisting fracture maybe caused by the increase in loads due to one or more mechanisms, e.g., fluid pressure on the wall of wellbore and/or on crack surfaces, tectonic activity, or a long-wave source. The only requirement (condition) is that the loads be represented by the given stresses at infinity and/or given stresses or displacements at the boundaries of the domain under consideration.

Condition on the Fault For a natural fault we use the same type of the boundary conditions as for a rapidly pressurized cracks i.e., $\Delta u_{n}=0$ and there is Coulomb's friction. When under compression, the condition on the fault is usually described by Coulomb's law with cohesion $c$ and a friction angle $\rho$. If the normal traction on some part becomes tensile due to the influence of the approaching crack, the tractions are set to zero on that part to reflect its opening. To evaluate the role of friction, we consider Coulomb's relation without cohesion:

$$
\sigma_{n t}=f \cdot \sigma_{n n} \frac{\Delta u_{t}}{\left|\Delta u_{t}\right|}
$$




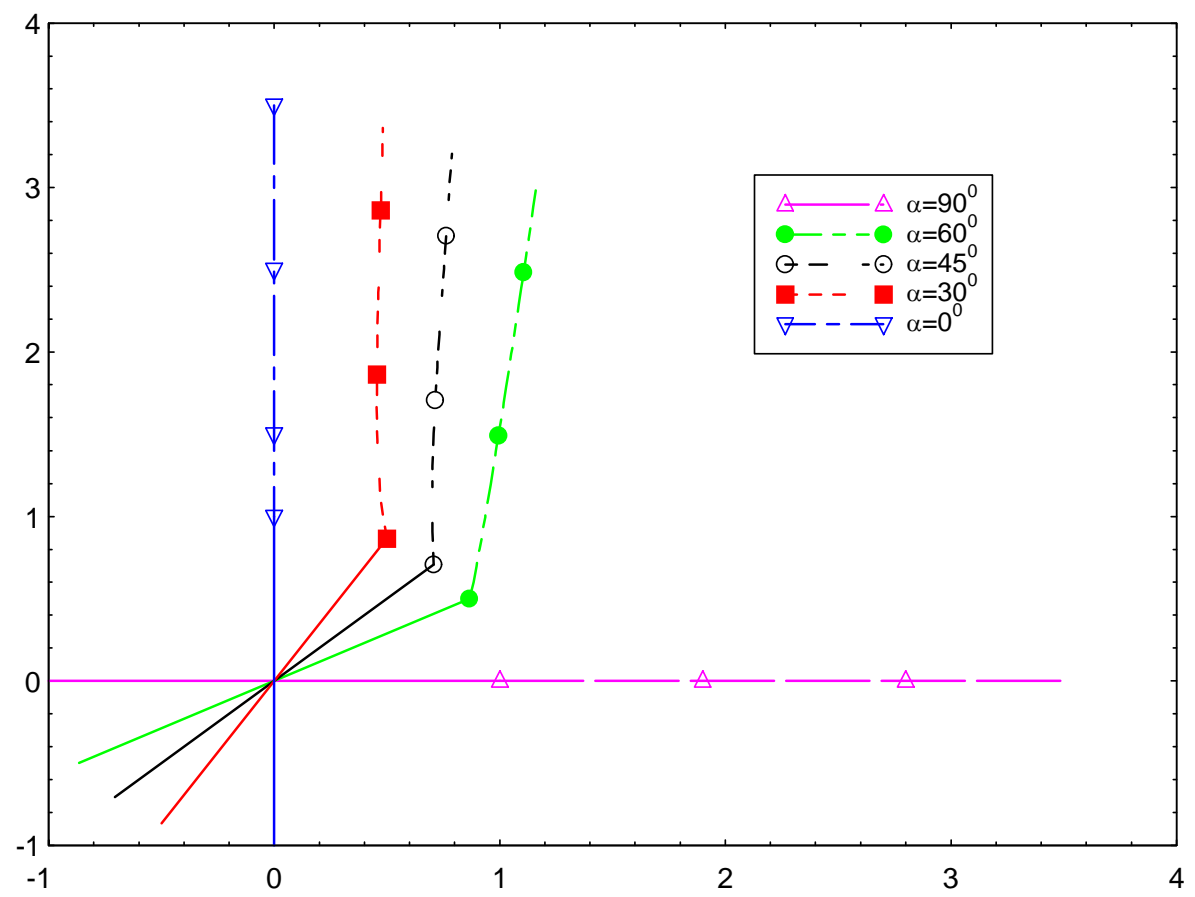

Figure 49: The trajectories of isolated cracks under fluid pressure (slow rate of loading).

where $\sigma_{n t}$ and $\sigma_{n n}$ are tangential and normal tractions on the fault surface, $\Delta u_{t}$ is the tangential displacement discontinuity (between opposite boundary points), $f=\tan \rho$, where $\rho$ is the angle of internal friction. This condition refers to the limiting case when the entire fault is in the plastic state. When searching for the crack trajectory in the close vicinity of a fault, a more general concept of interaction between the fault surfaces should be considered to include fault cohesion and its deterioration under loading. In this way, the stress is redistributed due to the approaching fracture, and due to the growing load, and the fault surface gradually is transferred into a sliding state. Of course, the case of open fracture should not be excluded a priori.

Fracture trajectory and the inclination of the preexisting crack We first consider the trajectory of a crack emanating from a wellbore and an isolated crack; and investigate the dependence of the fracture trajectory on the angle $\alpha$ measuring the inclination of the preexisting crack relative to the vertical axis (coinciding with the direction of the maximum principal stress at infinity). Then, we will study its behavior near a fault (Figure 48). For the isolated crack problem we consider the crack half-length to be $\ell=0.16$ $\mathrm{m}$ and assume that $K_{I C}=2 \mathrm{MPa} \sqrt{\mathrm{m}}$, and $\left(\sigma_{H}-\sigma_{h}\right)=2 \mathrm{MPa}$. When considering the wellbore/crack problem we assume that $\frac{\ell}{R}=0.1$ ( $\ell$ denoting the total crack length) and define a dimensionless parameter $\beta=\frac{\left(\sigma_{H}-\sigma_{h}\right) \sqrt{R}}{K_{I C}}$ controlling the fracture path [76]. For the first wellbore problem we have $\sigma_{H}=37.5 \mathrm{MPa}$ and $\sigma_{h}=25, \mathrm{MPa}$ and $K_{I C}=2 \mathrm{MPa} \sqrt{\mathrm{m}}$. In both problems, the fluid pressure is allowed to increase until the critical condition $K_{I}=K_{I C}$, thereafter it is held constant.

It can be seen in Figure 49 that as expected, the isolated crack turns in the direction of maximum in-situ 


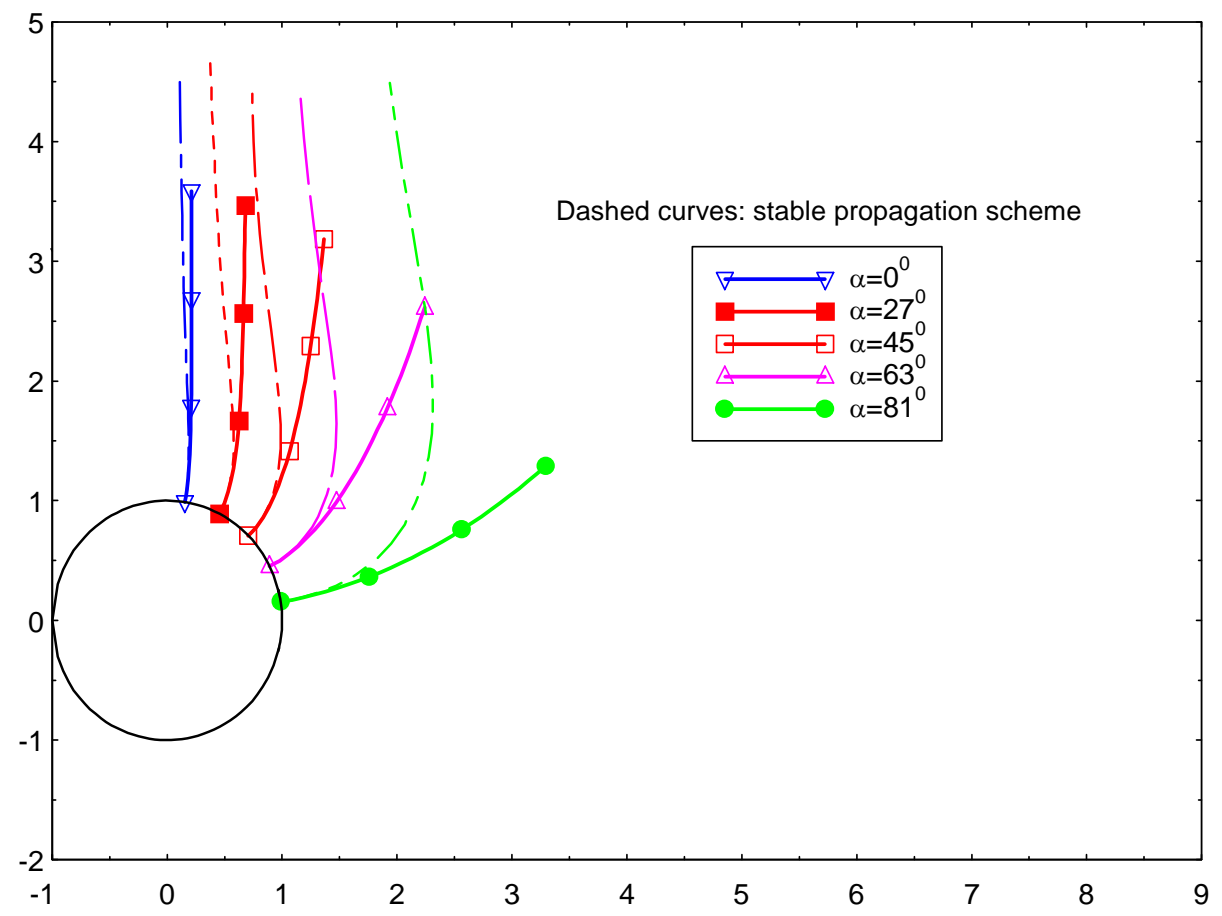

Figure 50: Fractures trajectories for differenet inclinations of the preexisting crack (slow pressurization, $\beta=3.95)$. 


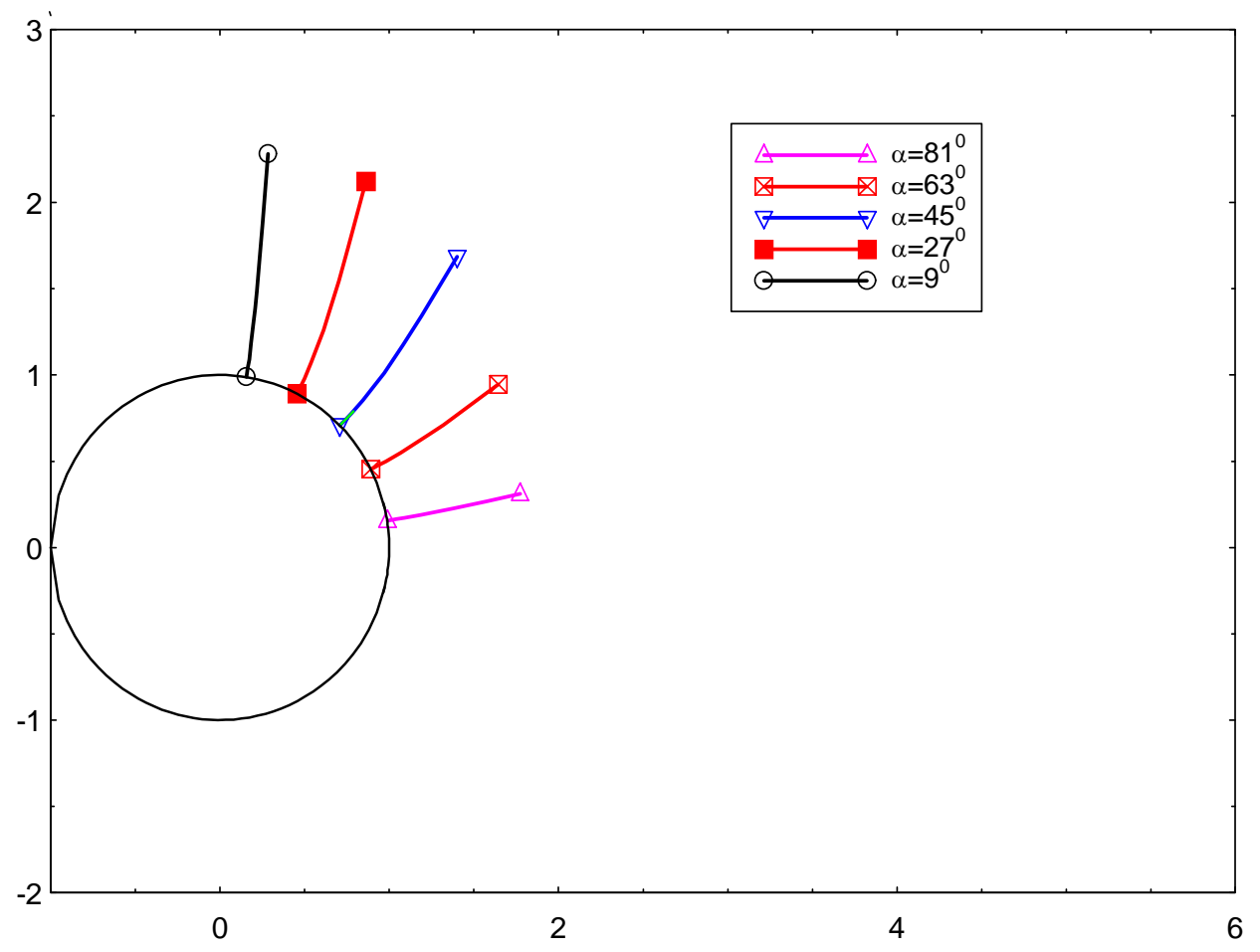

Figure 51: Fractures trajectories for different inclinations of the preexisting crack (fast pressurization, $\beta=$ $3.95)$. 
stress. This example dealt with the simplest case of an unstable crack configuration $\left(\alpha=90^{\circ}\right)$ and the results could be expected by taking into account the symmetry of the geometry and the loading conditions. Below we shall consider a more complicated problem.

Figures 50-51 refer to the cases of slow and fast pressurization. The behavior of the cracks agrees well with those reported by [76] for fast pressurization i.e., the trajectory generally maintains its initial direction. The differences for the slow pressurization cases are due to the fact we are considering unstable crack growth herein. In the opposite case, one can observe the trajectories turning to the direction of the maximum in situ stress. ${ }^{1}$.

Interaction of a crack and a fault In this section we study fracture propagation near a natural discontinuity. This is an important problem in modeling hydraulic fracture propagation in a naturally fractured reservoir. As before, we define a parameter $\beta=\frac{\left(\sigma_{H}-\sigma_{h}\right) \sqrt{d}}{K_{I C}}$ with $d=1.5 \ell \sqrt{2}$, where $\ell$ is the half-length of the preexisting crack, consider $\sigma_{H}$ and $\sigma_{h}$ to be constant, and increase the fluid pressure up to a critical value to satisfy the condition $K_{I}=K_{I C}$. For the examples of this section we let $\left(\sigma_{H}-\sigma_{h}\right)=2 \mathrm{MPa}$ and consider frictionless sliding of the fault. Thereby, this example is the case of slow pressurization rate so that the crack surfaces are completely opened by the fluid pressure.

Figures 52-56 illustrate the simulation results for a crack propagating in the vicinity of a fault. It is evident that the fault, as a surface with tangential displacement discontinuity (slip), modifies the stress field near the crack tip and the crack opening. Therefore, we observe deflections of the fractures trajectories from the direction of maximum far-field stress when they approach the fault. Figure 52 corresponds to a fault that is inclined to the $y$-axis by an angle $\varphi=45^{\circ}$, the distance between the center of the preexisting crack $(\ell=0.56 \mathrm{~m})$ and the fault is given by $d=\sqrt{2}(1.5)(0.56)$. We see that the fractures trajectories change considerably due to the presence of the fault. There is no tendency for them to turn towards the $y$-axis (compare with Figure 49). The next example (Figure 53) illustrates the role of the steepness of the fault. Now the angle $\varphi=18^{0}$, the effects of sliding become more important. One can observe that there is an angle $\alpha_{*}\left(75^{0}<\alpha_{*}<90^{0}\right)$ which determines the unstable crack configuration; for $\alpha=\alpha_{*}$, small disturbances of the parameters lead to a sharp deflection of the propagation trajectory.

Figure 54 demonstrates the influence of the initial crack length. We have the same angle $\varphi=18^{0}$ as in the previous case, but $\ell$ is now $0.06 \mathrm{~m}$ and $d=\sqrt{2}(1.5)(0.06)$. There are no essential changes in the behavior of the cracks.

The next two graphs series (Figures 55-56) depict the trajectories corresponding the case of $\left(\sigma_{H}-\sigma_{h}\right)=6$ MPa. The other parameters are equal to the ones of the previous case (Figure 53 and 54, respectively). As expected, the essential difference consists only in more sharp turning of the trajectories for the new series when $\left(\sigma_{H}-\sigma_{h}\right)=6 \mathrm{MPa}$. It should be noted that if we change the direction of $\sigma_{H}$ and $\sigma_{h}$, in the case of $\varphi=45^{\circ}$, the complete scheme and as a result, the trajectories rotate by an angle of $90^{\circ}$. For the case of $\varphi=18^{0}$ the situation is not so trivial. One can expect that the cracks will more readily follow the far-field stress direction, the results are shown in Figure 57.

The influence of friction Figures 58-59 depict the results of modeling the fractures trajectories near a frictional fault whose surfaces experience sliding. The friction parameter $f$ is equal to 0.5 and 2 , respectively. The geometric features of the propagation scheme are the same as that in Figure 53. We see that the friction

\footnotetext{
${ }^{1}$ T he marks on the graphs on figures 1-2 and consequent ones serve only to identify the curves.
} 


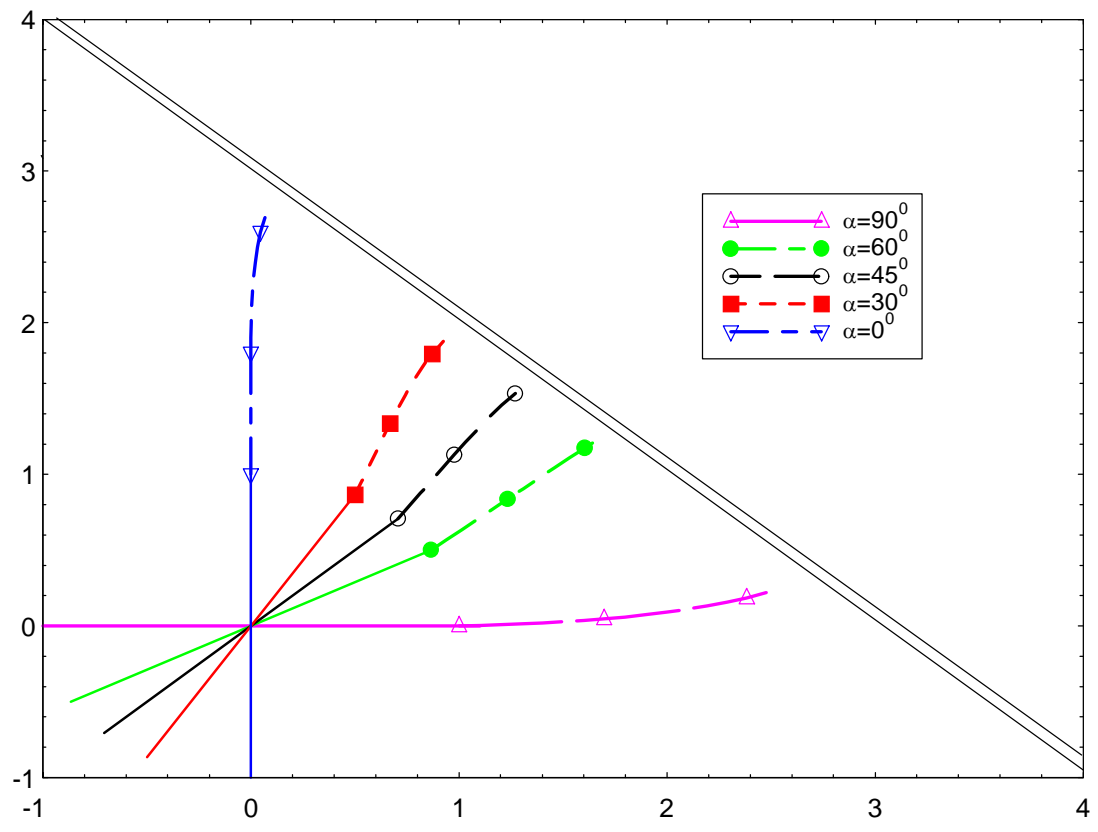

Figure 52: Fractures trajectories in the close vicinity of a gently inclined fault with $\sigma_{H}-\sigma_{h}=2 \mathrm{MPa}$, $\beta=1.19$. 


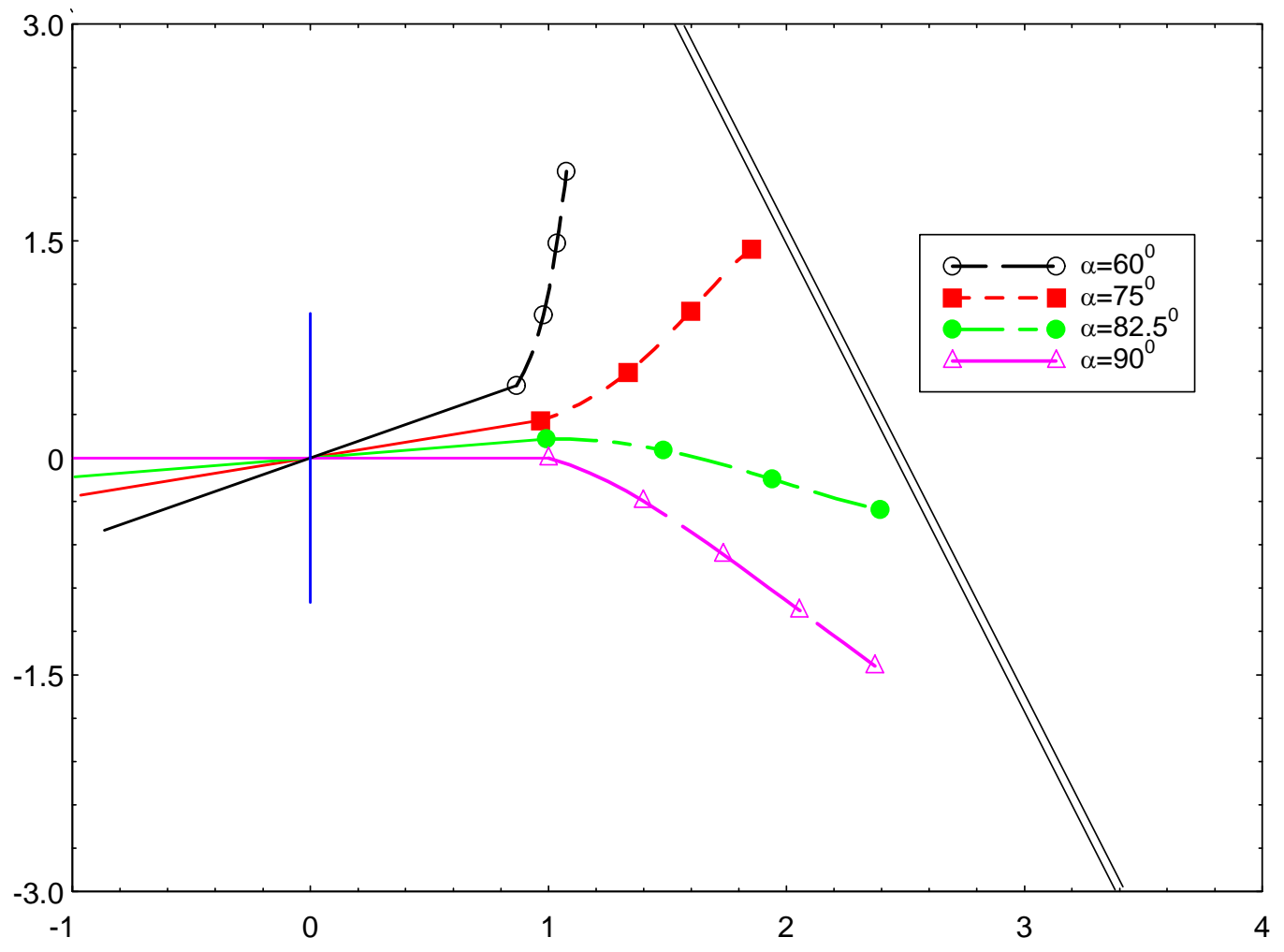

Figure 53: Fractures trajectories in the close vicinity of a steep fault, $\ell=0.56 \mathrm{~m}, \sigma_{H}-\sigma_{h}=2 \mathrm{MPa}$. 


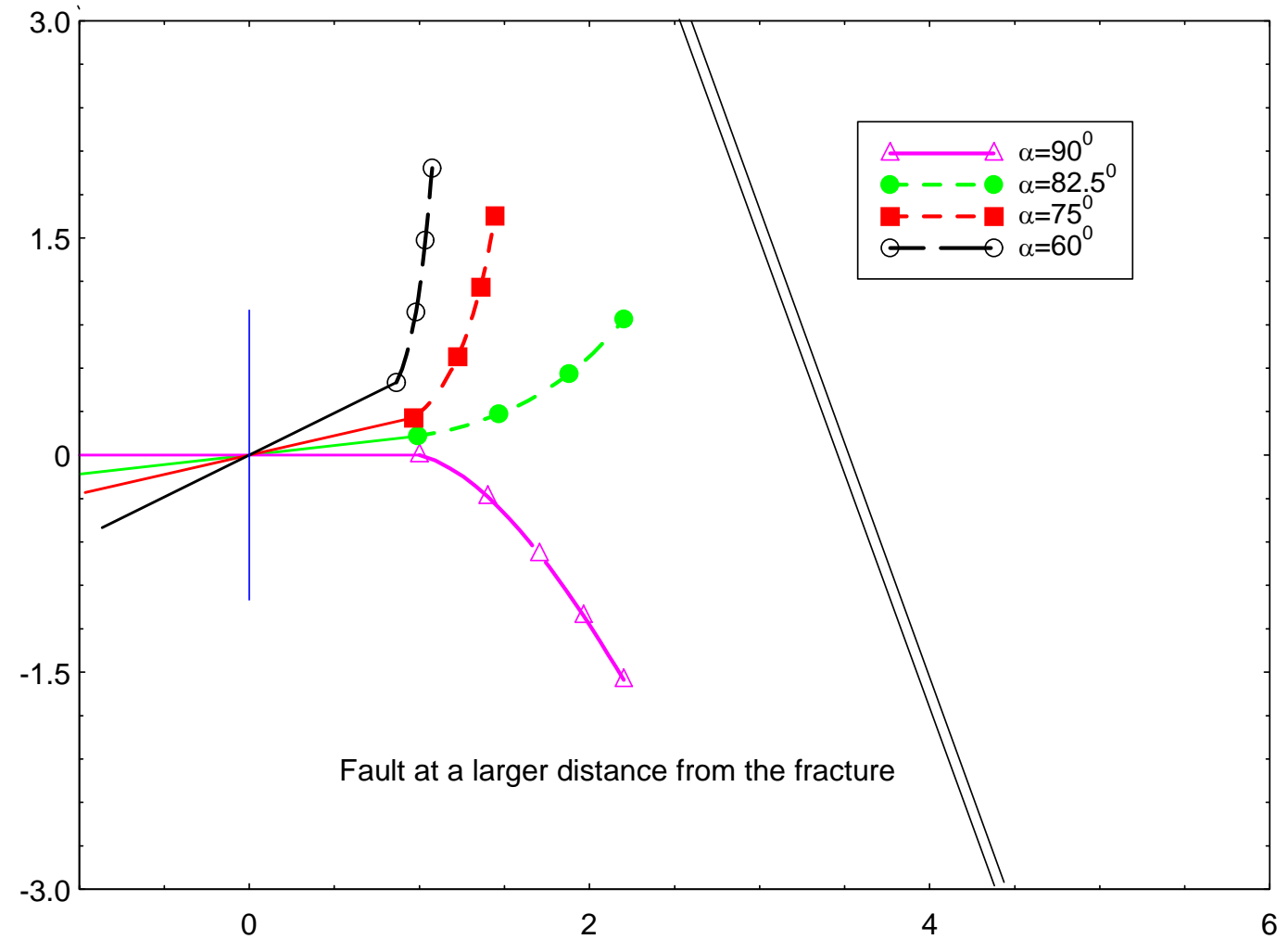

Figure 54: The role initial crack length, $\ell=0.06 \mathrm{~m}, \sigma_{H}-\sigma_{h}=2 \mathrm{MPa}$. 


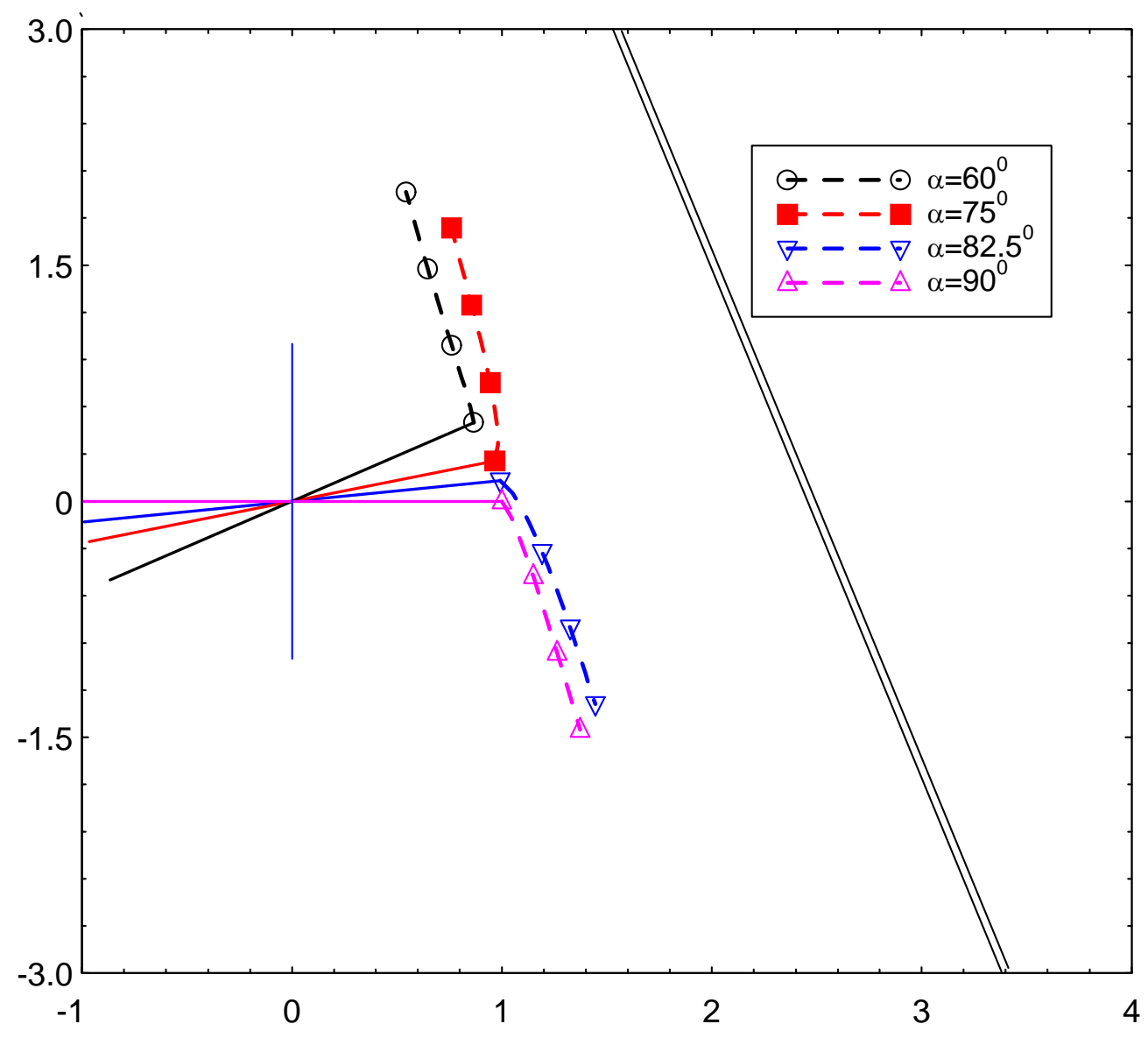

Figure 55: Trajectories due to the modification of the stresses, $\sigma_{H}-\sigma_{h}=6 \mathrm{MPa}, \ell=0.56 \mathrm{~m}$ (compare with Figure 53). 


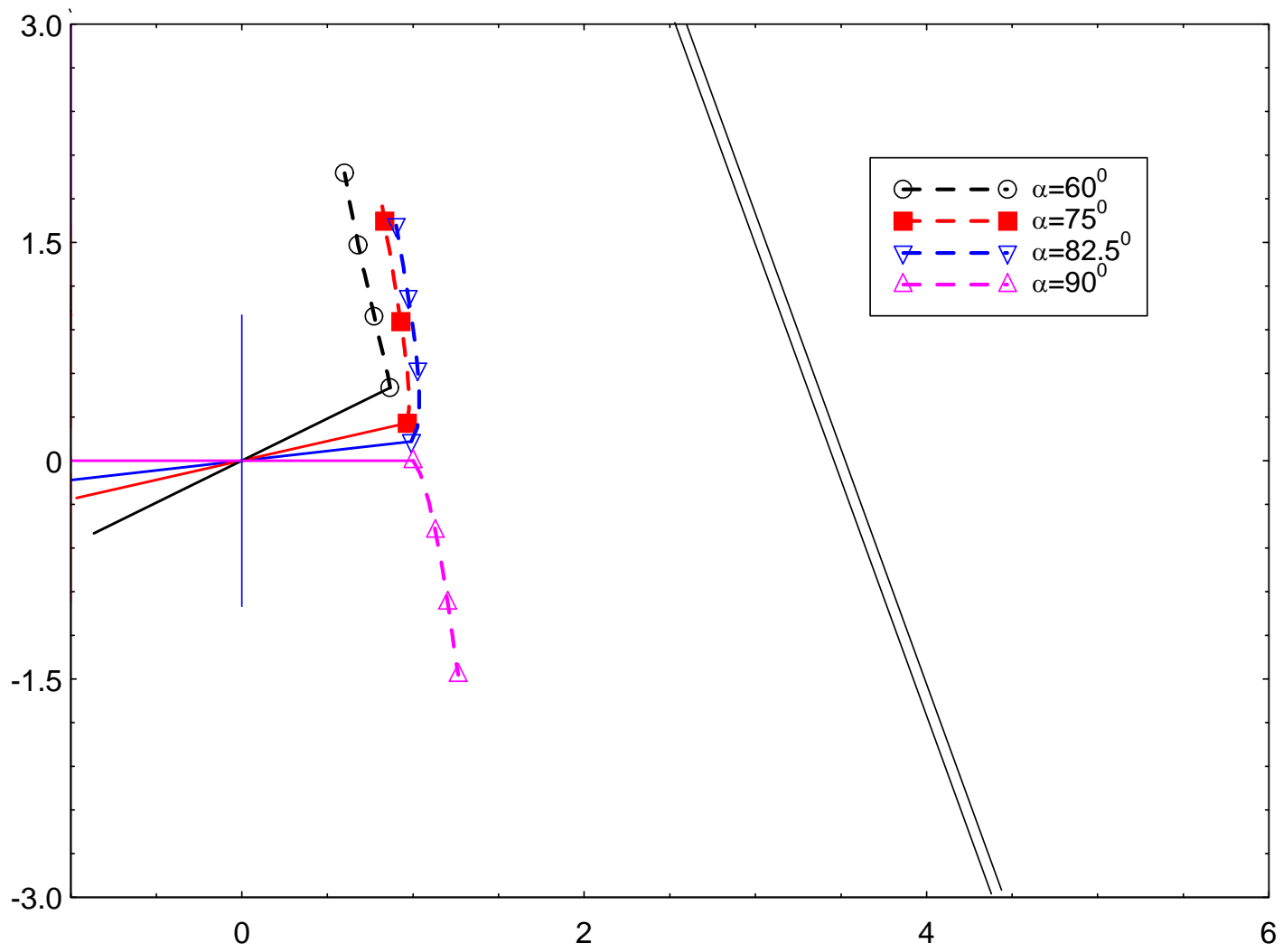

Figure 56: Trajectories near a steep fault at a larger distance, with $\sigma_{H}-\sigma_{h}=6 \mathrm{MPa}$ and $\ell=0.06 \mathrm{~m}$ (compare with Figure 54). 


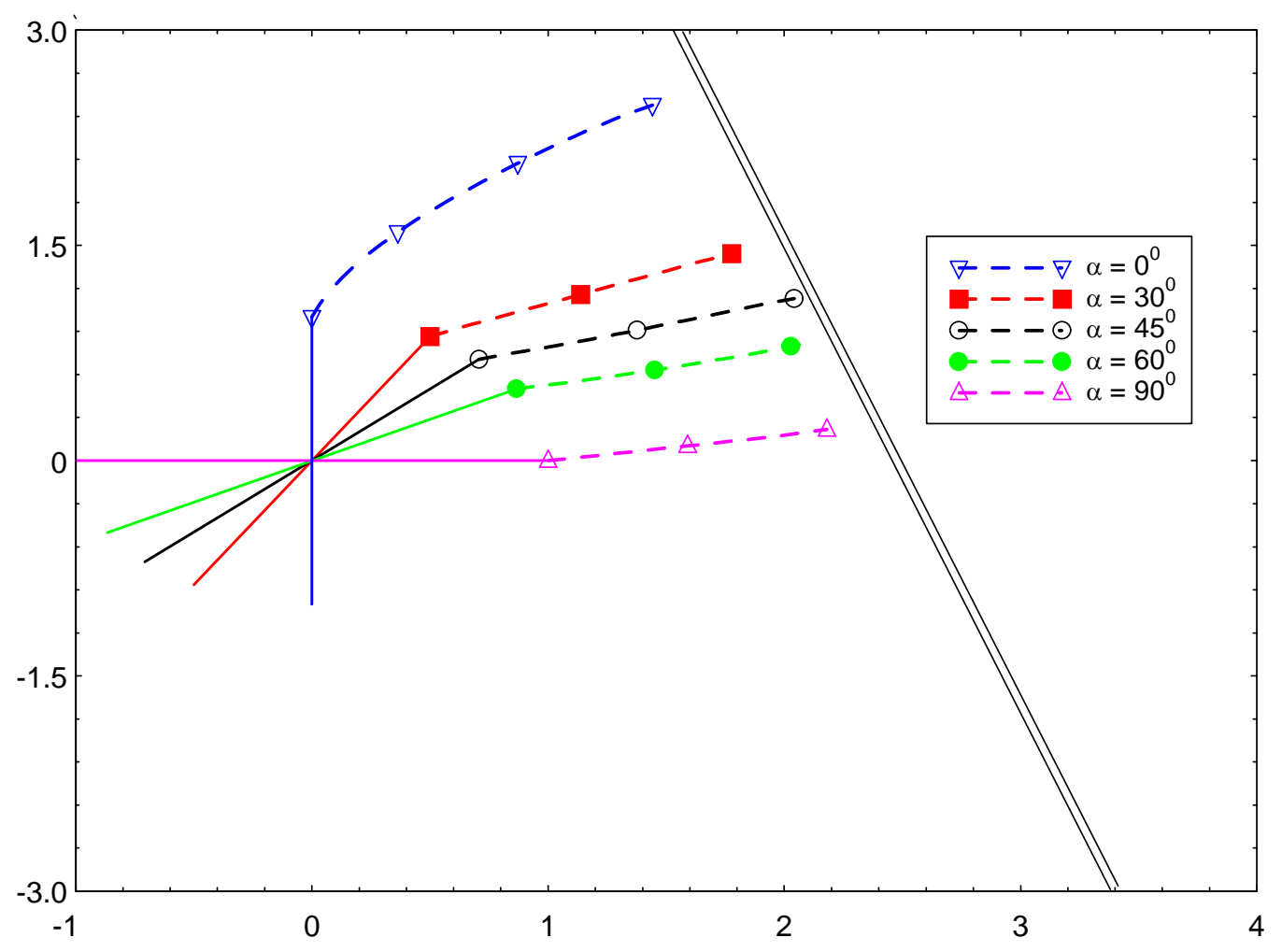

Figure 57: Crack paths for the case when the maximum stress is in the x-direction. 


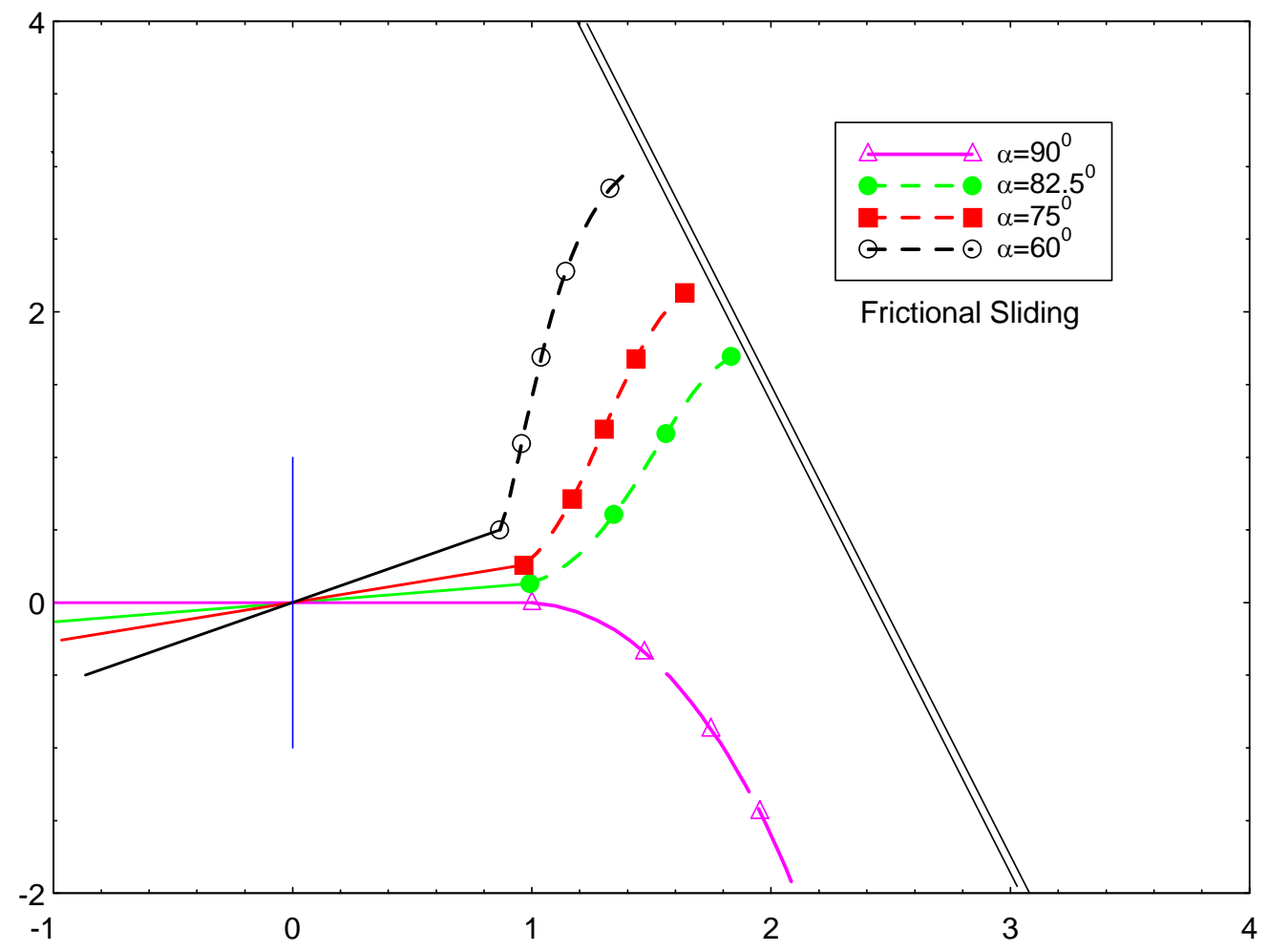

Figure 58: The effect of friction on the fracture path (small friction angle). 


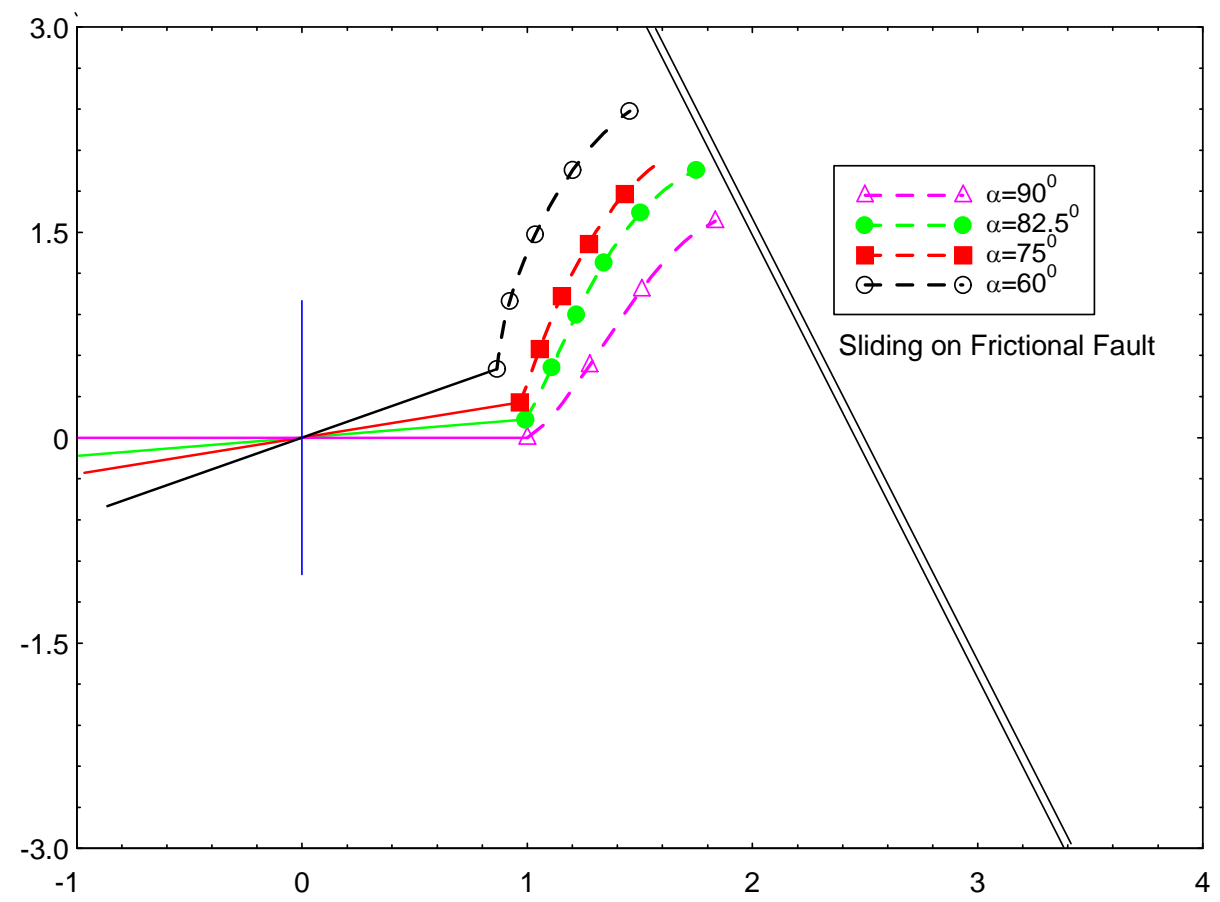

Figure 59: The effect of friction on the fracture path (larger friction angle). 


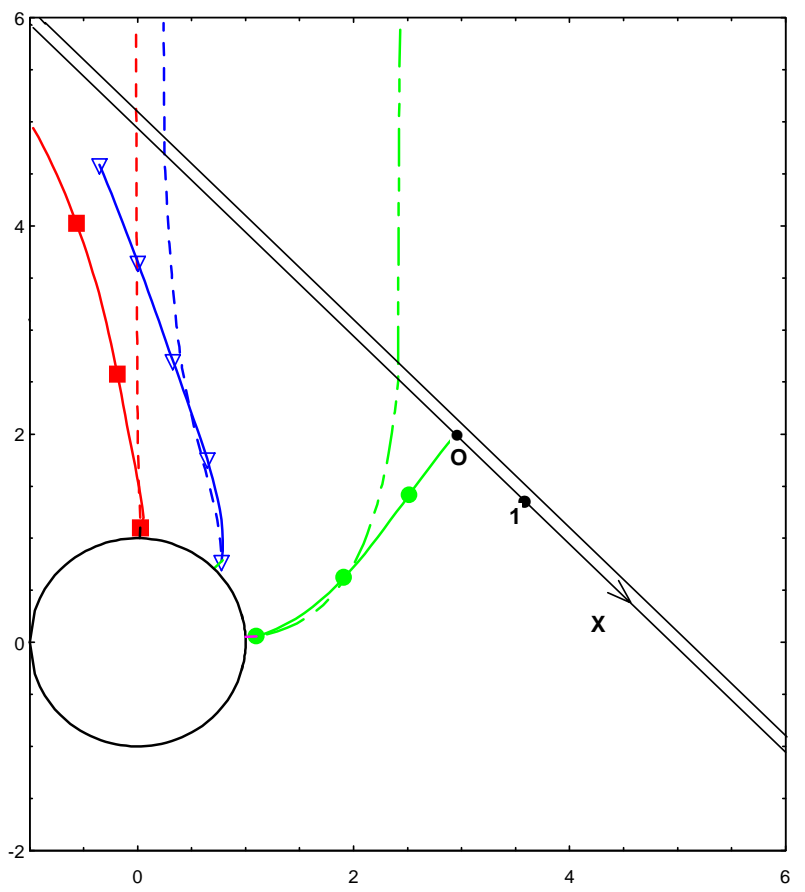

Figure 60: Crack propagation from a wellbore near a fault, $\beta=3.95$ (compare with Figure 50).

leads to the rotation of the trajectories towards the $x$-axis promoting intersection with the fault. Also, the critical inclination angle of the preexisting crack is more than that in the frictionless case; $82.5^{0}<\alpha_{*}<90^{0}$ for $f=0.5$.

It should be mentioned that the effects of sliding strongly depend on the angle $\varphi$ and the ratio $\frac{\sigma_{H}}{\sigma_{h}}$. The elementary static analysis leads to this conclusion. In the case of symmetry when $\varphi=0^{0}$ or $90^{\circ}$ and the fracture propagates normal to the fault, there is no sliding at all.

Crack emanating from a wellbore in the vicinity of a fault Now we modify the schemes of the previous section and consider the wellbore, crack, and fault interaction problem (Figure 48). Again, we vary the inclination of a preexisting crack relative to the vertical axis, $\alpha$, and assume that the wellbore radius is $R=0.1 ; \ell=0.01 \mathrm{~m}, K_{I C}=0.5 \mathrm{MPa} \cdot \sqrt{\mathrm{m}}$; and $\sigma_{H}=22, \sigma_{h}=11 \mathrm{MPa}$. The fluid pressure is allowed to increase until the critical condition $K_{I}=K_{I C}$ is satisfied, thereafter it is kept constant during fracture propagation. Figures 60-61 illustrate the crack trajectories near a fault under the same loading path as in Figure 52 and 53, respectively.

Note that new trajectories deviate more noticeably near the well and turn to become oriented parallel to the fault. Intersection with the fault is not promoted for initial cracks that are not sub-parallel to the $\mathrm{x}$ direction. However, as shown in Figure 63 (compare with Figure 62) when the wellbore is rapidly pressurized the cracks tend to follow their initial direction and possibly intersect the fault. The dashed lines in the first two figures show the fracture paths in the absence of a fault. 


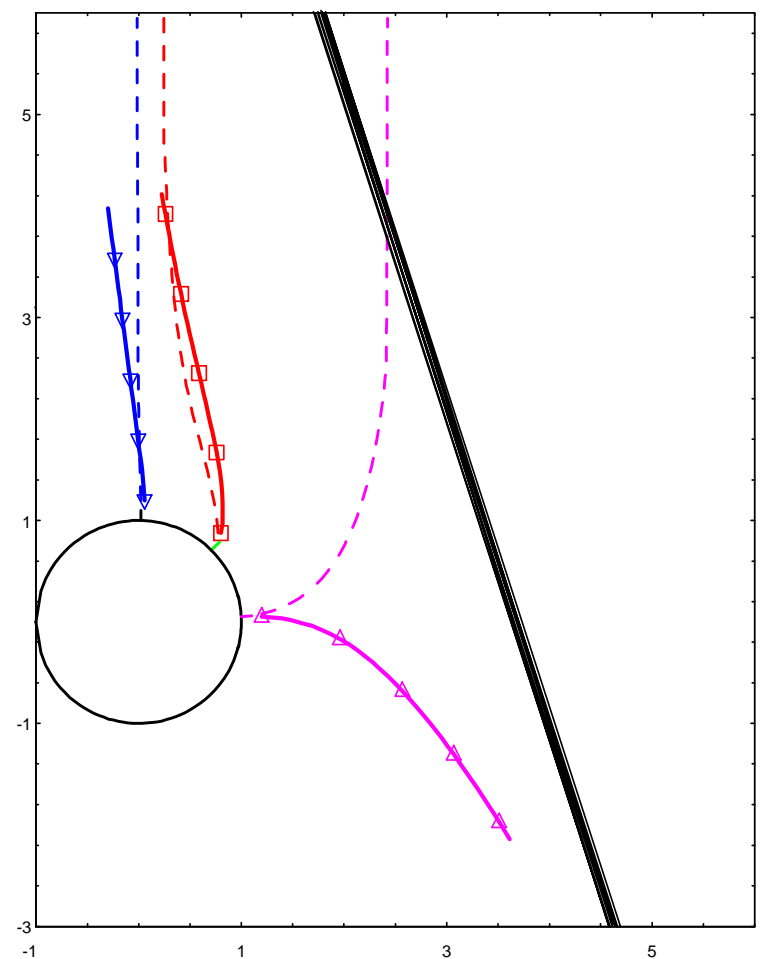

Figure 61: Crack emanating from a wellbore near a steep fault, $\beta=3.95$. 


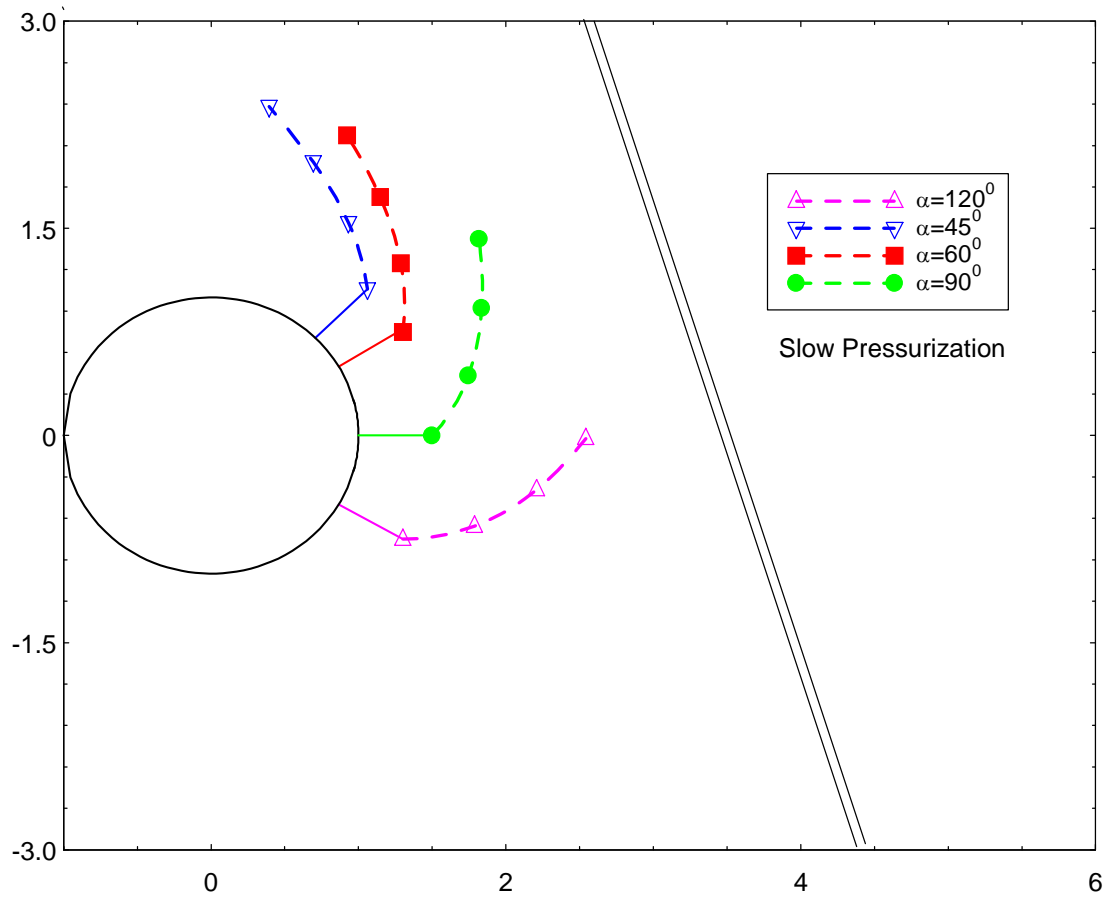

Figure 62: Crack paths for the case of slow pressurization, with $\sigma_{H}=15 \mathrm{MPa}, \sigma_{H}=10 \mathrm{MPa}, K_{I C}=2$ $\mathrm{MPa} \cdot \mathrm{m}^{\frac{1}{2}}(\beta=0.79)$. 


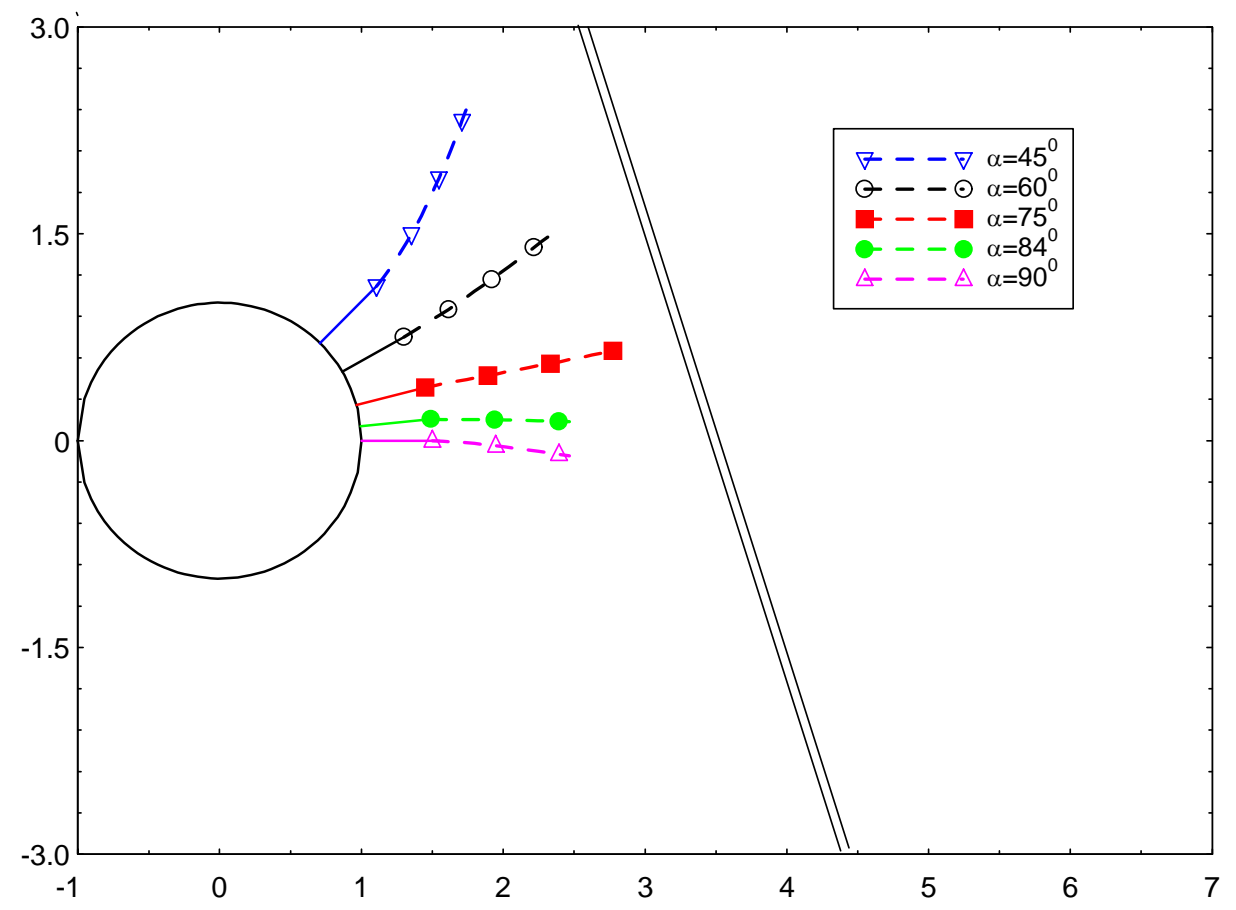

Figure 63: Crack paths for fast pressurization rate, with $\sigma_{H}=15 \mathrm{MPa}, \sigma_{H}=10, \mathrm{MPa}, K_{I C}=2 \mathrm{MPa} \cdot \mathrm{m}^{\frac{1}{2}}$ $(\beta=0.79)$. 
The conclusions are summarized as follows:

- in all the cases studied, both with and without a fault, the trajectory of the crack tends to be aligned in the direction of the maximum compressive principal stress acting in the vicinity of a wellbore

- a fault influences the crack propagation by altering the stresses in the vicinity of the wellbore from those at infinity

- a propagating crack noticeably influences tractions and displacement discontinuities on a fault when approaching at distances less than the wellbore radius

- the initial parts of the trajectories, obtained as explained by using the conditions 68-70 differ from those obtained from 68 and 69.

It is desirable to model the actual intersection of fractures to determine if the crack will cross the fault. However this is a complex problem. Firstly, it is necessary to have special (non-square root) asymptotics to calculate DD and SIF's for the small distances between the crack tip and the fault. Another question is the existence of an adequate criterion (based on experiments) for determining whether the crack penetrates or terminates at the interface. At last, one should be able to arrange the algorithm for tracing the path close to the fault such that the crack length increment should not intersect the fault. At the present we can only forecast whether the intersection is possible at all (we observe that sometimes crack apparently turns parallel to the fault).

\subsubsection{Implications for geothermal reservoir stimulation}

The analyses of Section 4 demonstrate a number of practical guidelines for geothermal reservoir development:

- Drilling: the drilling of a wellbore is a continual motion (ideally) that leads to gradual deepening of the wellbore. Initially, the relatively cool mud (and bit) comes in contact with increasingly warmer rocks (with depth). This leads to cooling of the formation near the wellbore. Cooling reduces the total stresses in the rock near the well (reducing the stress difference, i.e., Mohr's circle radius) and induces a pore pressure drop inside the formation that increases the effective stresses near the wellbore. As time increases, the mud temperature will equilibrate with its surroundings so that the formations higher in the section being drilled are subjected to the increased temperature of the mud. Heating generates increased pore pressure and total stresses near the wellbore, the former reduces the effective stresses. These processes may be occurring simultaneously in the lower and upper segments of the wellbore, respectively. Cooling can cause wellbore stability with respect to shear failure and instability with respect to tensile failure. The latter mechanism is useful in fracture initiation in stimulation; cold fluid injection can also significantly enhance fracture permeability.

- Fracture initiation/propagation: Modeling the propagation of fractures with various lengths and orientations clearly demonstrates that wellbore orientation can have an impact on future simulation practices. Trajectories of inclined wells need to be optimized in relation to future stimulation needs and the current state of stress to facilitate fracturing. Also, perforations need to be oriented favorably with respect to the in-situ stress direction. Perforations oriented close to the direction of maximum in-situ stress minimize near wellbore restrictions and can facilitate hydraulic stimulation [94]. 
- Fracturing of naturally-fractured reservoirs: Hydraulic fracturing has can be successfully applied in naturally fractured reservoirs. It has been observed that the path of the fracture is not a single plane but involves propagation along and through existing natural fractures [95]. However, a hydraulic fracturing treatment may not be successful if the existing fracture orientations are parallel to the current maximum horizontal stress. In this situation, the hydraulically induced fractures will most likely propagate parallel to the natural fractures with little connectivity with fractured zones. It may be better to orient the wellbore to intersect the natural fractures in the rock mass. In general, whether an artificial fracture can effectively propagate through a fractures rock mass depends on the joint properties, the in-situ stress state, and injection rate. Competent natural fractures with high normal stresses promote fractures intersection and crossing. The analysis shows that when the fluid does not penetrate the fracture, it tends not to be reoriented in the direction of maximum stress and may intersect existing natural cracks. Such pressurization scheme can be achieved in sleeve fracturing and dynamic fracturing by explosives. In an extensively fractured reservoirs, hydraulic stimulation can be used to connect the wellbore to the permeable fractured zones.

- Influence of cold fluid injection: The results have also shown that cold fluid injection in the reservoir can alter the stresses significantly and reduce the effective normal stress on joints. As a result, fracture slip is highly likely and can lead to permeability enhancement. Extensive slip, however, may cause a reduction of fracture permeability by crushing the aspirities. Furthermore, because the breakdown pressure is reduced by the cooling-induced tensile tangential stresses, reservoir cooling can impact interpretation of hydraulic fracturing for determination of in-situ stress. This needs to be considered in calculating the maximum in-situ stress. Furthermore, cooling a larger region around the wellbore will reduce the stresses so that using the instantaneous shut-in pressure will underestimate the value of the minimum in-situ stress. The latter should be considered when measuring stresses in hole that has been drilled for other purposes.

The models developed in this study allow one to perform quantitative analyses when considering these various issues in planning to develop an enhanced geothermal system.

\subsection{Graphics User Interface}

Experience has shown that an appropriate medium through which models can be applied and results studied is paramount to successful utilization of advanced scientific knowledge and technical solutions. Therefore, in order to maximize the efficacy to the industry, a graphics user interface (GUI) has been developed to enhance the fracture propagation modeling capabilities.

\subsubsection{General concepts \& characteristics}

As the operating system provides an environment for using the system efficiently, a means is necessary to facilitate user interaction with the software. The process of prompting the user to run each module separately, determine the individual results and infer the overall result of a simulation can be rather encumbered. Thus, it is better to provide the user with software that integrates the separate modules into a single entity. Wrapping the software with a Graphical User Interface will further enhance the user's ability to fully explore the software capabilities. In the worst possible case, the user of the application might not even know the sequence of execution of individual modules. Also, prompting the user to enter the input parameters during 
the execution of the program requires a prior knowledge of the flow of the program. However, building a GUI makes this unnecessary. This is achieved by providing the user with a list (sometimes broken into many) of input parameters on a screen that allow the user to view the input parameters in a glance. The input parameters are usually broken down into meaningful "classes". The parameters that are related to each other form a "class". One or more such classes can be grouped together and placed on a screen. The classes that have a loose binding can be separated into different screens. There are many criteria for binding these classes onto a single screen. If each class has many input parameters associated with it, and if two or more of such classes are placed on a single screen, then the screen becomes overloaded with parameters and might cause inconvenience to the user. At the same time, having a single screen for each class (if there are more than 10 screens) may also cause inconveniences for the user. The method of addressing the input parameter is also a key aspect of the screen. Some of the commonly used user interface components are text field, drop-down boxes, and choice buttons. Each of these has a particular appeal that depends on a number of factors. Therefore, factors that should be considered in building a GUI are numerous. However, the GUI should guide a novice user to understand the software. The GUI should guide the user through the process, anticipate the user's next move in the application, and provide choices. In some cases, the output generated by a program is best understood when it is viewed in its "raw" form. A "raw" output can be anything from a string of characters to a series of numbers. However, often times a graphical representation of the same "raw" output provides more information. The GUI can provide for graphical representation of raw data in the form of two-dimensional graphs, line graphs, bar graphs, and in tabular format. In short, GUI is a graphical representation of the software that gives it a friendly look and helps the user to experience the "feel" and "power" of the software.

The system architecture and the GUI have been described in detail elsewhere (NDRM-00-04, NDRM00-05, NDRM-01-06, Appendix C). Briefly, the user interface has graphics routines, and dialog boxes for conveniently selecting a desired program and entering data. Graphics capabilities have been implemented to assist the user in problem definition. Post-processing routines allow program output to be viewed directly through graphic presentations or saved in data files for later use.

\subsubsection{The client-server architecture}

The GUI for the fracture propagation model has been developed within the framework of a client-server model. In a typical case, client-server architecture consists of one server and one or more clients. The server,

as the name suggests, provides services to clients, the nature of which depends on the application. The services can range from performing a simulation requested by the client to "asking" other client(s) connected to that system to conduct simulations and to provide the results to the client. This is called a distributed computing system since there is a group of computers connected together and working "for" each other, with the server managing all the ongoing network activities.

If an application needs to plot graphs using a set of values, then the application can be broken into two parts: a part that calculates the necessary values and the other part which plots them. The function (or program) that performs the calculations can be placed on the server allowing the clients to implement the plotting function. Since the main function is placed on the server, the client needs only to log into the remote site and run the graphics application. Thus, the actual implementation of the application is transparent to the user. In this way, it is not necessary to distribute the program. However, a copy of the server program can be provided to the client through the download option. 
Two identifiers are needed to start communication with the server. The IP address which uniquely identifies the computer on the network (each unit on the network has an IP address). The other required parameter in a typical client-server application is the port number the server is running on (see Appendix B).

Description of the GUI for the fracture propagation code The current architecture of the crack propagation application is depicted in Appendix B. The flowchart explains the connectivity of the GUI and the program that actually generates the output files necessary for plotting and viewing the outcome of the experiment for the provided input parameters. The dashed-oval depicting the state "wait for completion" is entered when the user sends the input data to the server and is exited when the client receives a message from the server indicating that the server has completed the execution. It is at this stage that the client can download the output files from the server. This figure does not depict the order of execution of the application but only depicts the steps in which a user will follow to view the results of the provided input data with many or no intermediate steps. It can be observed that when the user sends the input data to the engine for processing, the application waits for either the new element's coordinates or an event indicating the end of the execution. If a new element was added, then it is plotted on the screen. After plotting an element, the program checks to see if the user has requested to "pause" the execution. If not, the application writes out a command to the engine to continue the execution, and so the engine goes on to the next iteration and adds another element, if any. If a request for a "pause" is issued, the client application enters a state where the intermediate data from the engine can be accessed. When the engine recognizes such a command from the user, it creates an intermediate data file that contains the information of all the elements that were added during the propagation since the beginning of the simulation, and sends out this file to the client application. The client application then displays this data to the user, who can make the desired modifications. Once the user has completed the editing, the data is transmitted back to the engine, which then runs the new data and the above cycle continues until the end of the execution.

Other aspects and details of the GUI's various options can be found in Appendix B. Below we illustrate the characteristics of the GUI through some technical examples.

\subsubsection{En echelon fracture}

Figure 64 illustrates a typical fracture interaction problem namely, the en echelon fracture geometry comprised of two cracks separated by some distance $(1 \mathrm{~cm}$ in this case). The lower crack is allowed to propagate under the influence of a far-field isotropic tensile stress. The fracture initially propagates in a straight direction, as it reaches the vicinity of the left tip of the upper fracture it turns away from it. However, as a result of mechanical interaction with the free face of the upper crack, it turns up and intersects the upper crack. This is in very good agreement with experimental observation (Thomas and Pollard, 1993).

\subsubsection{Fracture propagation from a wellbore}

The real time analysis of the hydraulic fracturing problem described previously is shown in Figure 65. The user can also study the pressure history and the stress intensity factor as the crack propagates. These are useful for monitoring stable and unstable fracture growth and pressure requirements. 


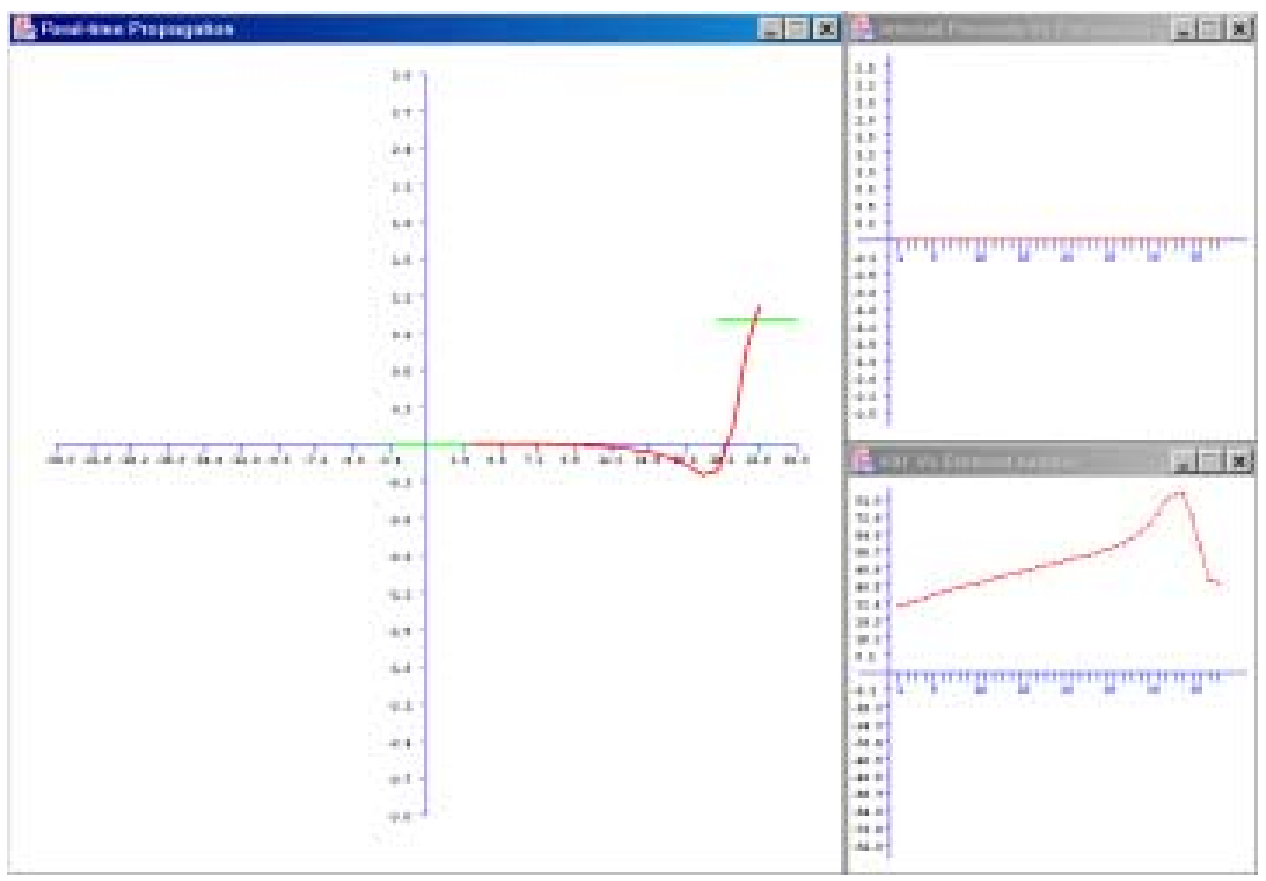

Figure 64: Real time propagation of en echelon cracks under isotropic tension.

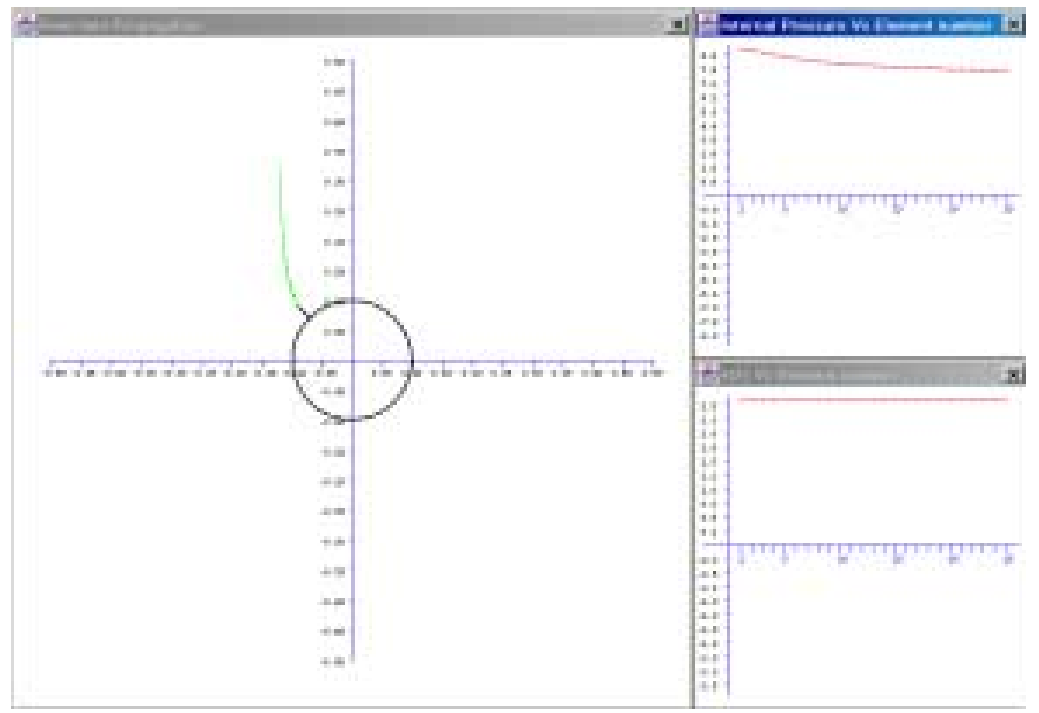

Figure 65: Hydraulic fracture propagation from a wellbore. Also shown, are the graphs of pressure and SIF history. 


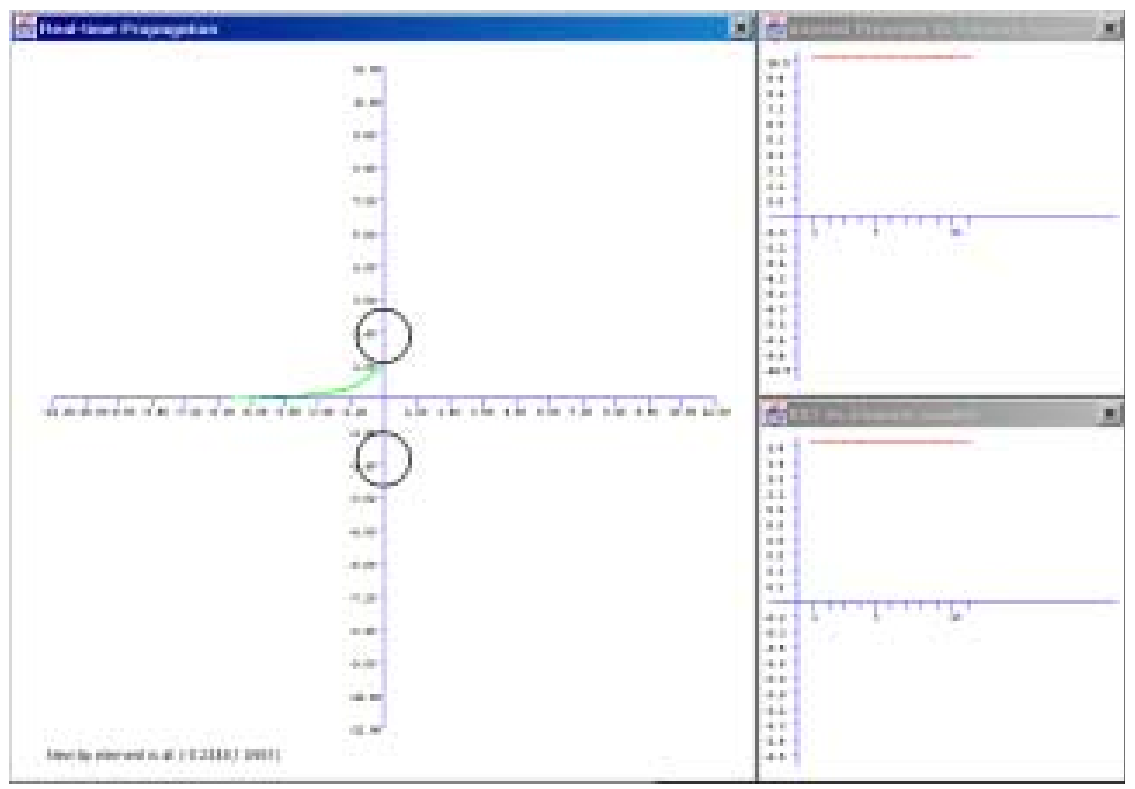

Figure 66: Fracture propagation in heterogeneous rock.

\subsubsection{Hydraulic fracture near solid inclusions}

To illustrate the capability of the model to consider rock heterogeneity, consider the influence of inclusions (e.g., rock grains or other material inhomogeneity) on fracture path (Figure 66). A hydraulically driven crack is approaching two inclusions in a compressive stress field with the major principal stress in the $x$ direction The upper inclusion is five times stiffer than the lower one, which has a stiffness that is twice that of the rock matrix. It can be seen that the crack deviates from the direction of maximum in-situ stress and tends to be attracted to the stiffer inclusion. This is due to the fact that the stiffer material carries the higher compressive stresses and modifies the stress distribution near it to promote easier propagation. The softer inclusion cannot carry as much load and transfers it to the material surrounding it resulting in higher compression zones not conducive to fracture growth.

\subsection{Heat Extraction}

\subsubsection{Solution of the general 1D model}

Before solving the governing equations of this problem we reduce the number of relevant variables by expressing them in non-dimensional form. First, we require that all lengths be normalized by the fracture length $L$

$$
\begin{aligned}
& x^{*}=x / L \\
& y^{*}=y / L
\end{aligned}
$$

and the time by the breakthrough time of the water flow $L / v$

$$
t^{*}=\frac{v t}{L}
$$


Temperature is replaced by the normalized temperature deficit

$$
\begin{aligned}
T_{w d}^{*} & =\frac{T_{r o}-T_{w}}{T_{r o}-T_{w o}} \\
T_{r d}^{*} & =\frac{T_{r o}-T_{r}}{T_{r o}-T_{w o}}
\end{aligned}
$$

Upon substitution in 31 and 32 we then obtain

$$
\frac{\partial T_{w d}^{*}}{\partial t^{*}}+\frac{\partial T_{w d}^{*}}{\partial x^{*}}-\frac{1}{P_{e}} \frac{\partial^{2} T_{w d}^{*}}{\partial x^{* 2}}=\left.S_{w} \frac{\partial T_{r d}^{*}}{\partial y^{*}}\right|_{y^{*}=0}
$$

and

$$
S_{r} \frac{\partial^{2} T_{r d}^{*}}{\partial y^{* 2}}=\frac{\partial T_{r d}^{*}}{\partial t^{*}}
$$

where

$$
P_{e}=\frac{v L}{D_{L}}
$$

is the Peclet number, and

$$
\begin{aligned}
S_{r} & =\frac{K_{r}}{v L \rho_{r} c_{r}} \\
S_{w} & =\frac{K_{r}}{v b \rho_{w} c_{w}}
\end{aligned}
$$

are equivalent to the Stanton number in heat transfer with $K_{r} / L$ and $K_{r} / b$ playing the role of heat transfer coefficients.

The two governing equations (75) and (76) need to be supplemented by initial and boundary conditions. Using the new variables, the conditions for $(75)$ are

$$
\begin{aligned}
T_{w d}^{*}\left(x^{*}, 0\right) & =0 \\
T_{w d}^{*}\left(0, t^{*}\right) & =1
\end{aligned}
$$

and for (76) we have:

$$
\begin{aligned}
T_{r d}^{*}\left(x^{*}, y^{*}, 0\right) & =0 \\
T_{r d}^{*}\left(x^{*}, 0, t^{*}\right) & =T_{w d}^{*}\left(x^{*}, t^{*}\right)
\end{aligned}
$$

The solution to three different versions of the problem are presented next. The second and third problems include additional phenomena that increase their level of complexity.

Model I First we re-visit Lowell's solution [81]. In this model, the dispersive transport term and transient storage term in (75) are neglected. Equation (75) simplifies to

$$
\frac{\partial T_{w d}^{*}}{\partial x^{*}}=\left.S_{w} \frac{\partial T_{r d}^{*}}{\partial y^{*}}\right|_{y^{*}=0}
$$


To solve this equation problem, the Laplace transform technique is utilized. Equations (83) and (76) then become

$$
\begin{gathered}
\frac{d \widetilde{T}_{w d}^{*}}{d x^{*}}=\left.S_{w} \frac{\partial \widetilde{T}_{r d}^{*}}{\partial y^{*}}\right|_{y^{*}=0} \\
S_{r} \frac{\partial^{2} \widetilde{T}_{r d}^{*}}{\partial y^{* 2}}=s \widetilde{T}_{r d}^{*}
\end{gathered}
$$

where the tilde denotes Laplace transform, and $s$ is the Laplace transform parameter. Solution of (85) with B.C. (82) is

$$
\widetilde{T}_{r d}^{*}=\widetilde{T}_{w d}^{*} \exp \left(-\sqrt{\frac{s}{S_{r}}} y^{*}\right)
$$

Substituting (86) into (84) and using the B.C. (80), we find

$$
\widetilde{T}_{w d}^{*}=\frac{1}{s} \exp \left(-\sqrt{\frac{s}{S_{r}}} S_{w} x^{*}\right)
$$

The inverse Laplace transform yields:

$$
T_{w d}^{*}=\operatorname{erfc}\left(\frac{1}{\sqrt{t_{D}}}\right)
$$

where

$$
t_{D}=\frac{\bar{\beta} t^{*}}{x^{* 2}}
$$

and

$$
\bar{\beta}=\frac{4 S_{r}}{S_{w}^{2}}=\frac{4 v^{2} b^{2} \rho_{w}^{2} c_{w}^{2}}{L K_{r} \rho_{r} c_{r}}=\frac{Q^{2} \rho_{w}^{2} c_{w}^{2}}{v L K_{r} \rho_{r} c_{r}}
$$

This shows that the solution is a function of a single variable $t_{D}$ only, which combines the spatial and time variables. This implies that the solution is self-similar.

Since $T_{w d}^{*}$ is a temperature deficiency, it is desirable to represent the solution in terms of its complementary part:

$$
T_{w}^{*}=1-T_{w d}^{*}=\frac{T_{w}-T_{w o}}{T_{r o}-T_{w o}}=\operatorname{erf}\left(\frac{1}{\sqrt{t_{D}}}\right)
$$

This solution is plotted in Figure 67 in semi-log scale. It demonstrates the pattern of temperature drop with time at a fixed (spatial coordinate) point.

Although the above single variable solution without any parameters is concise, it may be more useful to express it in terms of both time and spatial variables, $x^{*}$ and $t^{*}$. As can be observed from (88) and (89), a dimensionless parameter $\bar{\beta}$ will also be involved. Using specific data which are taken mostly from reference [85]

$$
\begin{aligned}
L & =1000 \mathrm{~m} ; \quad b=1 \mathrm{~cm} ; \\
Q & =1.45 \mathrm{~cm}^{2} / \mathrm{s} ; \quad v=0.725 \mathrm{~cm} / \mathrm{s} ; \\
\rho_{w} & =1.0 \mathrm{~g} / \mathrm{cm}^{3} ; \quad \rho_{r}=2.65 \mathrm{~g} / \mathrm{cm}^{3} ; \\
c_{w} & =1.0 \mathrm{cal} / \mathrm{g} \cdot{ }^{\circ} C ; \quad c_{r}=0.25 \mathrm{cal} / \mathrm{g} \cdot{ }^{\circ} \mathrm{C} \\
K_{r} & =6.2 \times 10^{-3} \mathrm{cal} / \mathrm{cm} \cdot \mathrm{s} \cdot{ }^{\circ} \mathrm{C}
\end{aligned}
$$




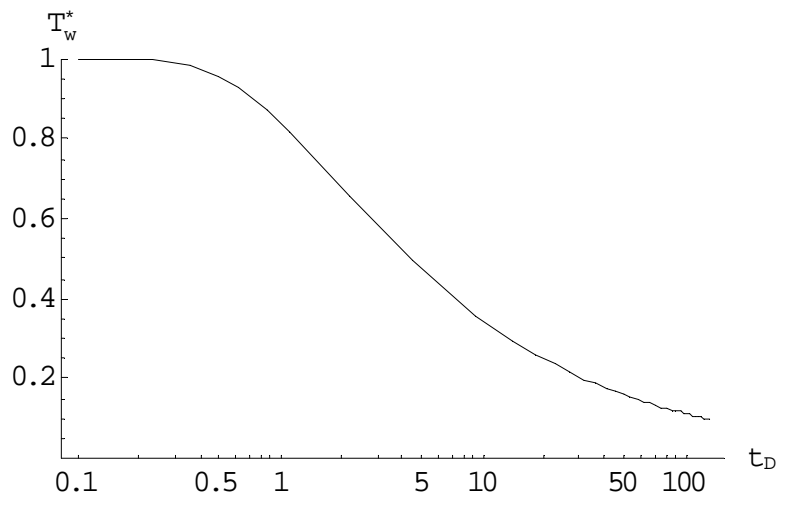

Figure 67: Plot of $T_{w}^{*}\left(t_{D}\right)$.

we find $\bar{\beta}=0.007$.

In Figure $68, T_{w}^{*}$ is plotted versus $x^{*}$ and $t^{*}$ for $\bar{\beta}=0.007$. The upper and lower diagrams show the result for $0 \leq t^{*} \leq 10$ and $0 \leq t^{*} \leq 1000$, respectively. We recall that $t^{*}=1$ marks the "breakthrough time" for the injected water. For the present set of data, the dimensional breakthrough time is 1.6 days. We observe that at $t^{*}=0$, the temperature in the fracture is that of the initial reservoir temperature, hence $T_{w}^{*}=1$. The upper diagram shows that as time increases, the temperature at the inlet $\left(x^{*}=0\right)$ drops immediately, while that at the outlet $\left(x^{*}=1\right)$ it is roughly at the initial reservoir temperature. The lower diagram demonstrates that after 4.4 years of operation $\left(t^{*}=1000\right)$, the increase of water temperature at the outlet decreases to about $40 \%$ of the extractable amount, $T_{r_{o}}-T_{w o}$.

Model II In this case, we retain the $\partial T_{w} / \partial t$ term in (75), while still neglecting the dispersion term:

$$
\frac{\partial T_{w d}^{*}}{\partial t^{*}}+\frac{\partial T_{w d}^{*}}{\partial x^{*}}=\left.S_{w} \frac{\partial T_{r d}^{*}}{\partial y^{*}}\right|_{y^{*}=0}
$$

Equation (76) remains the same. Upon transformation to Laplace space, we obtain

$$
\begin{aligned}
s \widetilde{T}_{w d}^{*}+\frac{d \widetilde{T}_{w d}^{*}}{d x^{*}} & =\left.S_{w} \frac{\partial \widetilde{T}_{r d}^{*}}{\partial y^{*}}\right|_{y^{*}=0} \\
S_{r} \frac{\partial^{2} \widetilde{T}_{r d}^{*}}{\partial y^{* 2}} & =s \widetilde{T}_{r d}^{*}
\end{aligned}
$$

The solution of the above equations with the boundary conditions (81)-(82) is:

$$
\widetilde{T}_{w d}^{*}=\frac{1}{s} \exp \left[-\left(\sqrt{\frac{s}{S_{r}}} S_{w}+s\right) x\right]
$$

The inverse Laplace transform produces

$$
\begin{aligned}
& T_{w}^{*}=\operatorname{erf}\left(\frac{1}{\sqrt{t_{D}^{\prime}}}\right) ; \quad \text { for } x^{*} \leq t^{*} \\
& T_{w}^{*}=1 ; \quad \text { for } x^{*} \geq t^{*}
\end{aligned}
$$



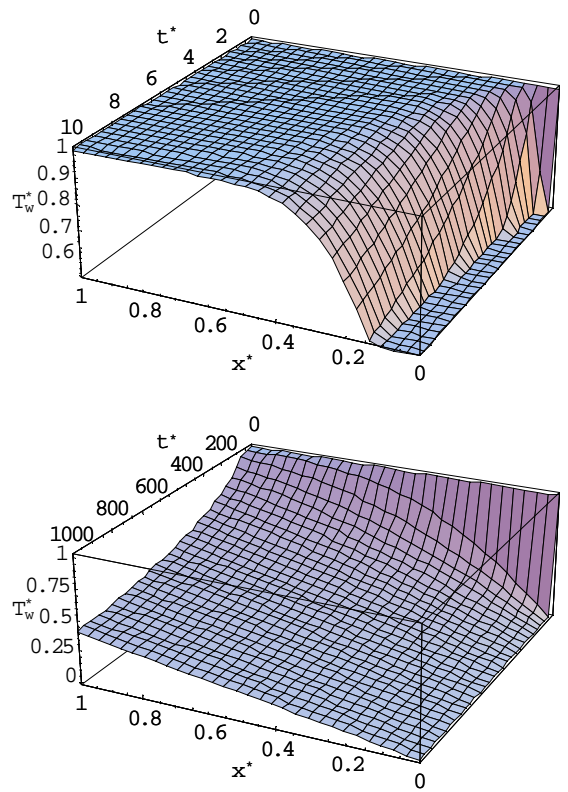

Figure 68: Plot of $T_{w}^{*}$ versus $x^{*}$ and $t^{*}$, for $\beta=0.007$.

where

$$
t_{D}^{\prime}=\frac{\beta\left(t^{*}-x^{*}\right)}{x^{* 2}}
$$

As before, the solution (96) can be expressed in terms of $x^{*}$ and $t^{*}$, for a given $\bar{\beta}$ value. For $\bar{\beta}=0.007$, however, the difference between the two solutions, (91) and (96), is negligibly small. As the value of $\bar{\beta}$ increases, the difference becomes more appreciable but is limited to small times. The difference between the two solutions for the case of $\bar{\beta}=0.1$ for $0 \leq t^{*} \leq 2$ is presented in Figure 69. This shows that under most circumstances the simplified model which neglects the time derivative term in $(75)$ is sufficiently accurate.

Model III In this model, the full governing equations (75) and (76) are used. Before proceeding with the solution, it would be illuminating to first examine the physical origin of the dispersion effect. Let us assume that the fracture flow takes place between two parallel plates. If the flow is laminar, the velocity profile is parabolic, as shown in Figure 70. However, the transport velocity $v$ used in the heat transport equation, is based on the average value of velocity over the fracture width. Comparing the two profiles in Figure 70, shows that a part of the heat will be transported at a velocity greater than the average, and a part will lag behind. This causes a stretching of heat in the longitudinal direction. An analogy exists for turbulent flow $[78,79]$. Hence we introduce a longitudinal dispersion term with the dispersion coefficient $D_{L}$.

After Laplace transform, (75) becomes

$$
s \widetilde{T}_{w d}^{*}+\frac{d \widetilde{T}_{w d}^{*}}{d x^{*}}-\frac{1}{P_{e}} \frac{d^{2} \widetilde{T}_{w d}^{*}}{d x^{* 2}}=\left.S_{w} \frac{\partial \widetilde{T}_{r d}^{*}}{\partial y^{*}}\right|_{y^{*}=0}
$$




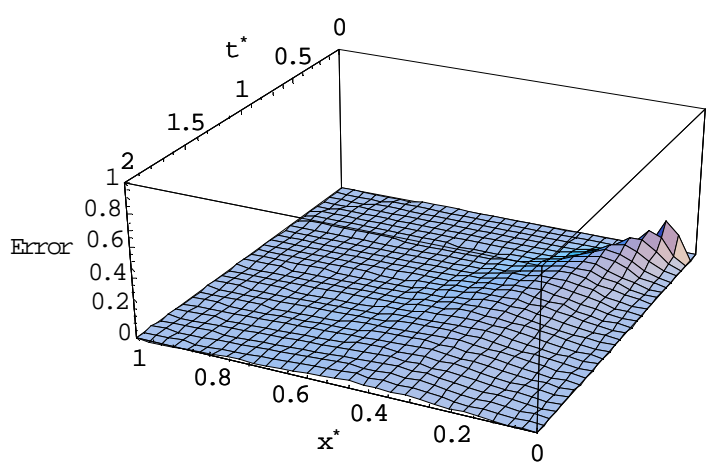

Figure 69: Difference of $T_{w}^{*}$ between Model I and Model II for the case $\bar{\beta}=0.1$.

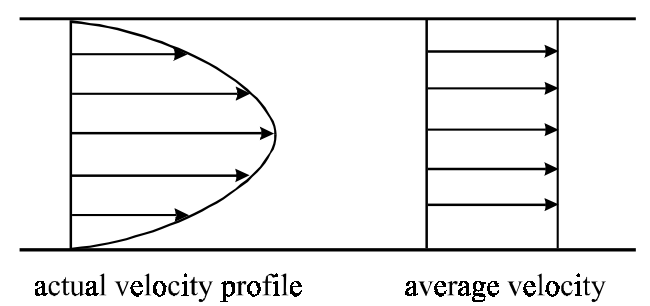

Figure 70: Velocity profile in parallel plates.

Substituting the solution of (85) into the right hand side of (98), we obtain the second order ODE:

$$
s \widetilde{T}_{w d}^{*}+\frac{d \widetilde{T}_{w d}^{*}}{d x^{*}}-\frac{1}{P_{e}} \frac{d^{2} \widetilde{T}_{w d}^{*}}{d x^{* 2}}=-2 \sqrt{\frac{s}{\beta}} \widetilde{T}_{w d}^{*}
$$

For the second order equation, a second boundary condition is required:

$$
\frac{\partial T_{w d}^{*}}{\partial x^{*}}=0 ; \quad \text { at } x^{*}=1
$$

The solution of (99) is:

$$
\widetilde{T}_{w d}^{*}=\frac{\lambda_{1} e^{\lambda_{1}+\lambda_{2} x^{*}}-\lambda_{2} e^{\lambda_{2}+\lambda_{1} x^{*}}}{s\left(\lambda_{1} e^{\lambda_{1}}-\lambda_{2} e^{\lambda_{2}}\right)}
$$

with

$$
\lambda_{1,2}=\frac{P_{e}}{2} \pm \frac{1}{2} \sqrt{P_{e}^{2}-4 P_{e}\left(s+2 \sqrt{\frac{s}{\beta}}\right)}
$$

Or, for its complementary part, we have:

$$
\widetilde{T}_{w}^{*}=\frac{1}{s}\left(1-\frac{\lambda_{1} e^{\lambda_{1}+\lambda_{2} x^{*}}-\lambda_{2} e^{\lambda_{2}+\lambda_{1} x^{*}}}{\lambda_{1} e^{\lambda_{1}}-\lambda_{2} e^{\lambda_{2}}}\right)
$$


In this case, we do not attempt to analytically invert the solution. Rather, numerical inversion [82] is performed using the Stehfest method.

The solution $T_{w d}^{*}$ is a function of the independent variables $x^{*}$ and $t^{*}$, and the parameters $\bar{\beta}$ and $P_{e}$. To evaluate the Peclet number, the longitudinal dispersion coefficient $D_{L}$ must be estimated. First, we check the Reynolds number:

$$
R_{e}=\frac{2 \rho_{w} v b}{\mu_{w}}
$$

where $\mu_{w}$ is the viscosity of water. Using the following data for water at $300^{\circ} \mathrm{C}$ :

$$
\begin{aligned}
\mu_{w} & =8 \times 10^{-4} \mathrm{~g} / \mathrm{cm} \cdot \mathrm{s} \\
K_{w} & =1.4 \times 10^{-3} \mathrm{cal} / \mathrm{cm} \cdot \mathrm{s} \cdot{ }^{\circ} \mathrm{C}
\end{aligned}
$$

yields $R_{e}=1800$. This is close to the critical Reynolds number of 2,000; hence flow is likely to be turbulent.

For laminar flow, we can use the following formula introduced by G.I. Taylor [78] for pipe flow:

$$
D_{L}=\frac{b^{2} v^{2}}{48 D}
$$

where

$$
D=\frac{K_{w}}{\rho_{w} c_{w}}
$$

is the heat diffusion coefficient. Based on the given data, we calculate $D=1.4 \times 10^{-3} \mathrm{~cm}^{2} / \mathrm{s}$ and $D_{L}=7.8$ $\mathrm{cm}^{2} / \mathrm{s}$. This gives $P_{e}=9.3 \times 10^{3}$. Comparison of the two solutions (96) and (103), however, shows that the dispersion effect is insignificant in the laminar flow range.

For turbulent flow, we may use the formula from reference [79], i.e.,

$$
D_{L}=10 \cdot a v^{*}
$$

where

$$
v^{*}=\sqrt{\frac{\tau_{o}}{\rho_{w}}}
$$

is the shear velocity. To calculate the shear velocity, the information of pressure drop across the fracture for a given flow rate is necessary. Since this data is not available, we shall use an arbitrary estimate. Assume that in turbulent flow the longitudinal dispersion coefficient is increased by 100 fold, giving a Peclet number $P_{e} \approx 100$. In this case, we find that there exists some small dispersion effect near the injection well at small times. But at the outlet, the difference in extraction temperature is negligible. Figure 71 shows the difference of the two solutions, (96) and (103), for $0 \leq t^{*} \leq 2$.

Influence of leakoff In this part we investigates the effect of fluid leakoff on heat extraction from a hot rock system. As a preliminary investigation, we shall make the assumption that both the heat conduction from the geothermal reservoir to the fracture fluid and the fluid loss into the reservoir is one-dimensional, perpendicular to the fracture (see Figure 72). 


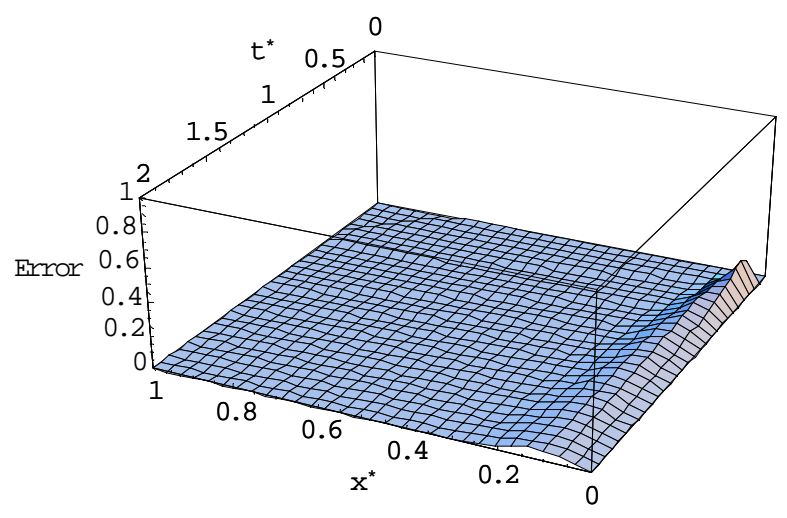

Figure 71: Difference of $T_{w}^{*}$ between Model II and Model III for the case $\bar{\beta}=0.007$ and $P_{e}=100$.

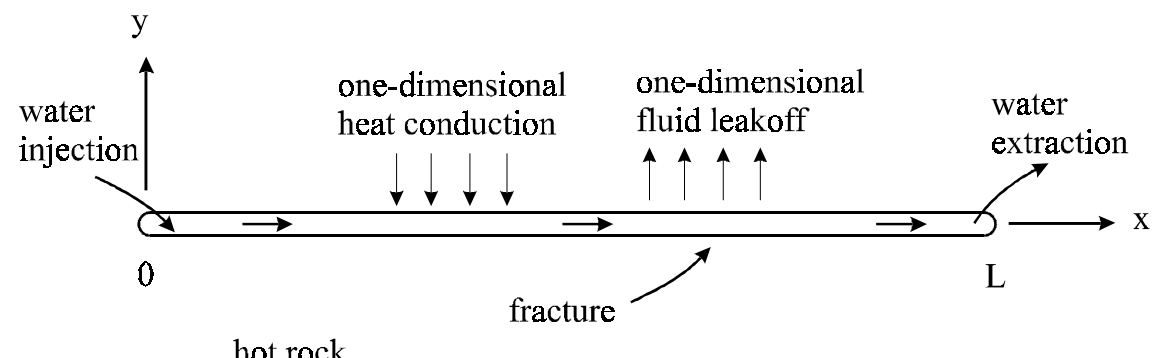

Figure 72: Geometry of mathematical model.

Figure 73 shows the heat balance in a segment of fracture. The heat transport equation in the fracture is then expressed as

$$
\begin{gathered}
-\rho_{w} c_{w} b \frac{\partial v(x) T(x, 0, t)}{\partial x}+\left.2 K_{r} \frac{\partial T(x, y, t)}{\partial y}\right|_{y=0^{+}} \\
-2 \rho_{w} c_{w} q(x) T(x, 0, t)=\rho_{w} c_{w} b \frac{\partial T(x, 0, t)}{\partial t}
\end{gathered}
$$

where $T=T(x, y, t)$ is the temperature in the reservoir, $T(x, 0, t)$ is the temperature at the fracture wall, which is the same as the water temperature in the fracture, $b$ is the fracture thickness, $\rho_{w}$ is the water density, $c_{w}$ is the specific heat of water, $v(x)$ is the cross-sectional average velocity of water in fracture, $q(x)$ is the leakoff velocity of water into the reservoir, which is assumed to be constant in time, and $K_{r}$ is the rock thermal conductivity. Assume that the fluid is incompressible, the fluid mass conservation requires:

$$
b \frac{d v(x)}{d x}+2 q(x)=0
$$

Combining (110) and (111), we obtain:

$$
-\rho_{w} c_{w} b v(x) \frac{\partial T(x, 0, t)}{\partial x}+\left.2 K_{r} \frac{\partial T(x, y, t)}{\partial y}\right|_{y=0^{+}}=\rho_{w} c_{w} b \frac{\partial T(x, 0, t)}{\partial t}
$$




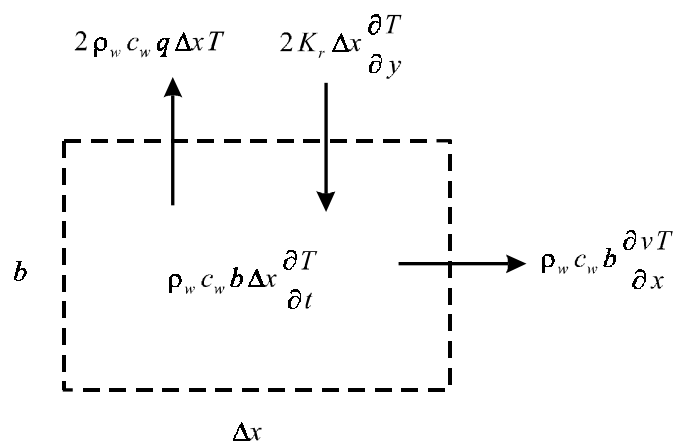

Figure 73: Heat balance in a fracture segment.

As we learned in the earlier report, the heat storage term on the right hand side has negligible effect in the advection dominant heat transport. Hence (112) is simplified to:

$$
\rho_{w} c_{w} b v(x) \frac{\partial T(x, 0, t)}{\partial x}=\left.2 K_{r} \frac{\partial T(x, y, t)}{\partial y}\right|_{y=0^{+}}
$$

Next we investigate the heat conduction in the rock. The one-dimensional heat transport equation is given by:

$$
\frac{\partial T(x, y, t)}{\partial t}+q(x) \frac{\partial T(x, y, t)}{\partial y}-\frac{K_{r}}{\rho_{r} c_{r}} \frac{\partial^{2} T(x, y, t)}{\partial y^{2}}=0
$$

where $\rho_{r}$ is the rock density, and $c_{r}$ is the specific heat of rock. The initial and boundary conditions are:

$$
\begin{aligned}
T(x, y, 0) & =T_{o} \\
T(0,0, t) & =T_{w}
\end{aligned}
$$

where $T_{o}$ is the initial reservoir temperature, $T_{w}$ is the injected water temperature. Equations (112), (113) and (114) then form the solution system.

It is however more convenient to use the dimensionless temperature deficit defined as

$$
T_{D}=\frac{T_{o}-T}{T_{o}-T_{w}}
$$

as the solution variable. This quantity has the limits $0 \leq T_{D} \leq 1$. Using (115) in (112) and (113), we obtain the form of governing equations:

$$
\begin{gathered}
\rho_{w} c_{w} b v(x) \frac{\partial T_{D}(x, 0, t)}{\partial x}=\left.2 K_{r} \frac{\partial T_{D}(x, y, t)}{\partial y}\right|_{y=0^{+}} \\
\frac{\partial T_{D}(x, y, t)}{\partial t}+q(x) \frac{\partial T_{D}(x, y, t)}{\partial y}-\frac{K_{r}}{\rho_{r} c_{r}} \frac{\partial^{2} T_{D}(x, y, t)}{\partial y^{2}}=0
\end{gathered}
$$

The initial and boundary conditions, however, becomes:

$$
\begin{aligned}
T_{D}(x, y, 0) & =0 \\
T_{D}(0,0, t) & =1
\end{aligned}
$$


The solution system them becomes (117), (118), and (119).

The first step of solution is to apply Laplace transform to the governing equations (117) and (118):

$$
\begin{gathered}
\rho_{w} c_{w} b v(x) \frac{\partial \widetilde{T}_{D}(x, 0, s)}{\partial x}=\left.2 K_{r} \frac{\partial \widetilde{T}_{D}(x, y, s)}{\partial y}\right|_{y=0^{+}} \\
s \widetilde{T}_{D}(x, y, s)+q(x) \frac{\partial \widetilde{T}_{D}(x, y, s)}{\partial y}-\frac{K_{r}}{\rho_{r} c_{r}} \frac{\partial^{2} \widetilde{T}_{D}(x, y, s)}{\partial y^{2}}=0
\end{gathered}
$$

The boundary condition is:

$$
\widetilde{T}_{D}(0,0, s)=\frac{1}{s}
$$

We first solve (121), which is a second order ODE. Its characteristic equation has two roots:

$$
\lambda_{1,2}=\frac{\rho_{r} c_{r} q(x)}{2 K_{r}}\left(1 \pm \sqrt{1+\frac{4 K_{r} s}{\rho_{r} c_{r}}}\right)
$$

By dropping the unbounded term, we obtain the solution as:

$$
\widetilde{T}_{D}(x, y, s)=\widetilde{T}_{D}(x, 0, s) \exp \left[\frac{\rho_{r} c_{r} q(x)}{2 K_{r}}\left(1-\sqrt{1+\frac{4 K_{r} s}{\rho_{r} c_{r} q^{2}(x)}}\right) y\right]
$$

Substituting (124) into (120), we obtain:

$$
\frac{\partial \widetilde{T}_{D}(x, 0, s)}{\partial x}=\frac{\rho_{r} c_{r} q(x)}{\rho_{w} c_{w} b v(x)}\left(1-\sqrt{1+\frac{4 K_{r} s}{\rho_{r} c_{r} q^{2}(x)}}\right) \widetilde{T}_{D}(x, 0, s)
$$

Equation (125) can be integrated. Together with the boundary condition, we find:

$$
\widetilde{T}_{D}(x, 0, s)=\frac{1}{s} \exp \left[\int_{0}^{x} \frac{\rho_{r} c_{r} q(x)}{\rho_{w} c_{w} b v(x)}\left(1-\sqrt{1+\frac{4 K_{r} s}{\rho_{r} c_{r} q^{2}(x)}}\right) d x\right]
$$

This gives the temperature distribution in the fracture. For temperature in the reservoir, we substitute (126) into (124).

The solution given (126) requires the knowledge of leakoff $q(x)$. For simplicity, we shall assume that the leakoff is constant along the fracture:

$$
q(x)=q_{o}
$$

From the continuity equation (111), we find the fracture flow velocity to be:

$$
v(x)=v_{o}-\frac{2 q_{o}}{b} x
$$

Substituting (127) and (128) into (126), we find:

$$
\widetilde{T}_{D}(x, 0, s)=\frac{1}{s}\left(1-\frac{2 q_{o}}{b v_{o}} x\right)^{-\frac{\rho_{r} c_{r}}{2 \rho_{w} c_{w}}\left(1-\sqrt{1+\frac{4 K_{r} s}{\rho_{r} c_{r} q_{o}^{2}}}\right)}
$$




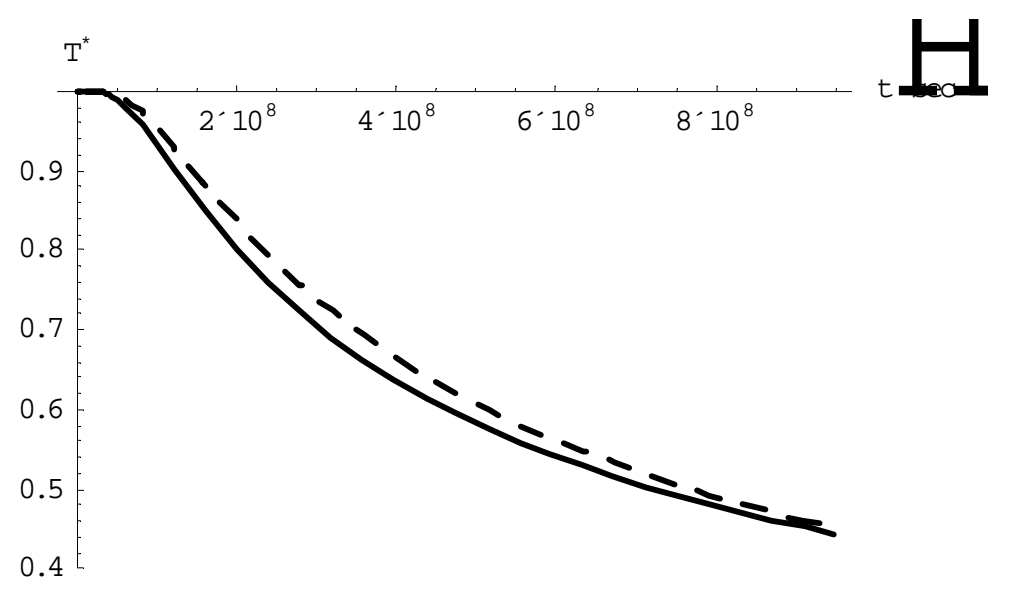

Figure 74: Extraction Temperature $T^{*}$ as function of time, at $20 \%$ fluid loss (dash line) and no fluid loss (solid line).

Approximate Laplace inversion, such as the Stehfest inversion can be used to invert the solution to the time domain.

For comparison purpose, we also present the solution without leakoff, i.e., $q_{o}=0$. In this case, the solution is given by:

$$
\widetilde{T}_{D}(x, 0, s)=\frac{1}{s} \exp \left(-\sqrt{\frac{4 K_{r} \rho_{r} c_{r} s}{\rho_{w}^{2} c_{w}^{2} b^{2} v_{o}^{2}}} x\right)
$$

Analytical inversion can be performed in this case, and the solution is:

$$
T_{D}(x, 0, t)=\operatorname{erfc}\left[\frac{x}{v_{o} b \rho_{w} c_{w}} \sqrt{\frac{K_{r} \rho_{r} c_{r}}{t}}\right]
$$

The temperature in the reservoir is:

$$
T_{D}(x, y, t)=\operatorname{erfc}\left[\frac{\left(2 K_{r} L+v b \rho_{w} c_{w} y\right) x}{2 L v b \rho_{w} c_{w}} \sqrt{\frac{\rho_{r} c_{r}}{K_{r} t}}\right]
$$

This solution is known as the Lowell [1976] solution.

To demonstrate the solution, we shall use the following set of data:

$$
\begin{aligned}
\rho_{w} & =1.0 \mathrm{~g} / \mathrm{cm}^{3} ; \quad \rho_{r}=2.65 \mathrm{~g} / \mathrm{cm}^{3} ; \quad K_{r}=6.2 \times 10^{-3} \mathrm{cal} / \mathrm{cm} \cdot \mathrm{s} \cdot{ }^{\circ} C ; \\
c_{w} & =1.0 \mathrm{cal} / \mathrm{g} \cdot{ }^{\circ} C ; \quad c_{r}=0.25 \mathrm{cal} / \mathrm{g} \cdot{ }^{\circ} C ; \\
L & =300 \mathrm{~m} ; \quad b=0.3 \mathrm{~cm} ; \quad v_{o}=0.5 \mathrm{~cm} / \mathrm{s}
\end{aligned}
$$

This gives a water volume injection rate per unit height of fracture as $Q_{i}=v_{o} b=0.15 \mathrm{~cm}^{2} / \mathrm{s}$. The fluid loss ratio is given by:

$$
r=\frac{2 q_{o} L}{Q_{i}}=\frac{2 q_{o} L}{v_{o} b}
$$




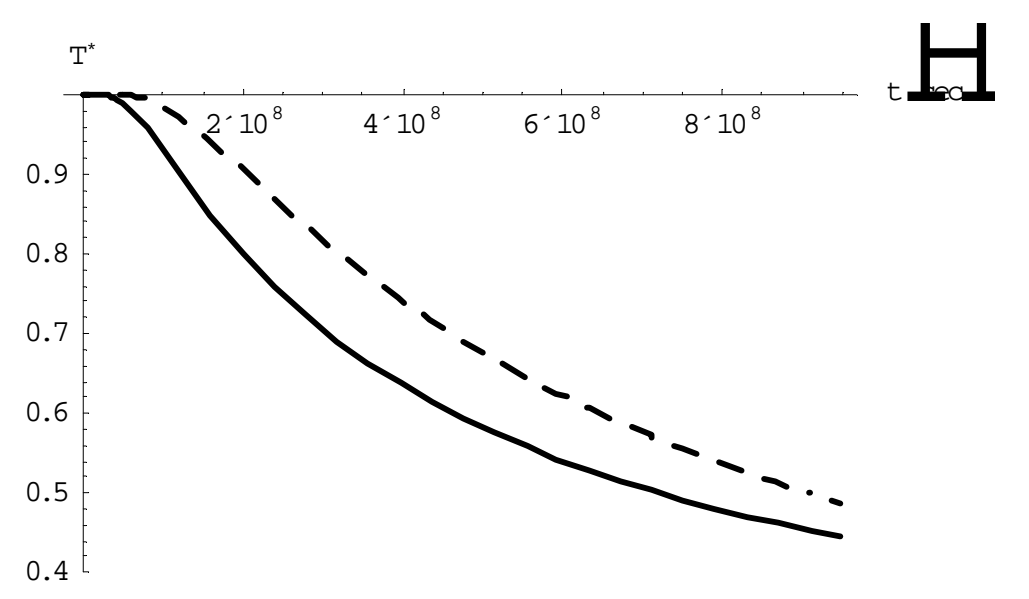

Figure 75: Extraction Temperature $T^{*}$ as function of time, at $50 \%$ fluid loss (dash line) and no fluid loss (solid line).

The extraction flow rate is:

$$
Q_{e}=(1-r) Q_{i}
$$

We shall present cases with different fluid loss ratio.

In Figure 74 and 75 we plot the normalized fluid extraction temperature:

$$
T^{*}(L, 0, t)=1-T_{D}(L, 0, t)=\frac{T(L, 0, t)-T_{w}}{T_{o}-T_{w}}
$$

for 30 years, respectively for fluid loss rate of $20 \%$ and $50 \%$. Also plotted is the Lowell solution, corresponding to no fluid loss. We observe that the extraction temperature is higher if the leakoff rate is larger, despite the fact that the formation is invaded by the lower temperature fluid. The higher temperature effect is caused by the decrease in fluid rate in the fracture due to leakoff. As fluid velocity decreases toward the outlet, the extracted fluid has longer residence time. To illustrate the residence time effect, we plot in Figure 76 the case without fluid leakoff, but with a $50 \%$ reduction of injection rate. The extraction temperature is significantly higher.

The effect of increased extraction temperature in the presence of fluid leakoff is somewhat misleading. Although the extraction temperature does increase, the total amount of heat extracted per unit time decreases because less amount of fluid is extracted. In Figure 77 we plot the rate of heat energy extracted per unit height of fracture for the $50 \%$ leakoff and no leakoff cases. Clearly the no leakoff case extracts more heat.

Next, we plot the temperature distribution in entire reservoir at a given time. In Figures 78 and 79 we plot $T^{*}$ in the range $0 \leq x \leq 300 \mathrm{~m}$ and $0 \leq y \leq 300$ at $t=30$ year, respectively for the $50 \%$ leakoff and no leakoff cases. We observe that with leakoff, the temperature is higher not only at the extraction point $(x, y)=(300 \mathrm{~m}, 0 \mathrm{~m})$, but also in the reservoir. This is another indication that less heat is extracted from the reservoir at that time.

Heat extraction from a fracture was studied in detail. After a careful investigation of the previously neglected effects of heat storage and heat dispersion within the fracture, it can be concluded that these effects are indeed negligible under ordinary conditions. 


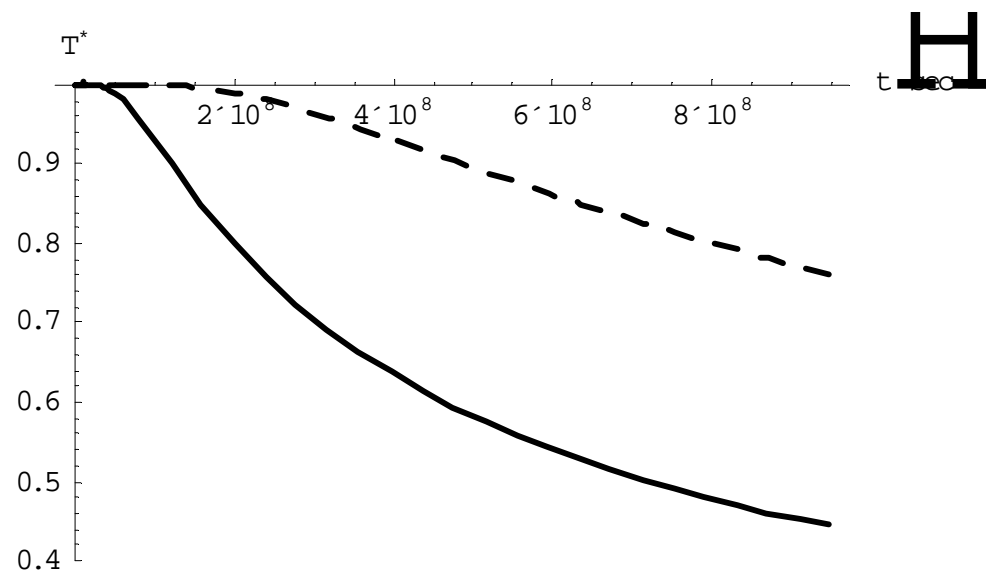

Figure 76: Extraction temperature $T^{*}$ as function of time, with $50 \%$ injection rate without leakoff (dash line), and full injection rate without leakoff (solid line).

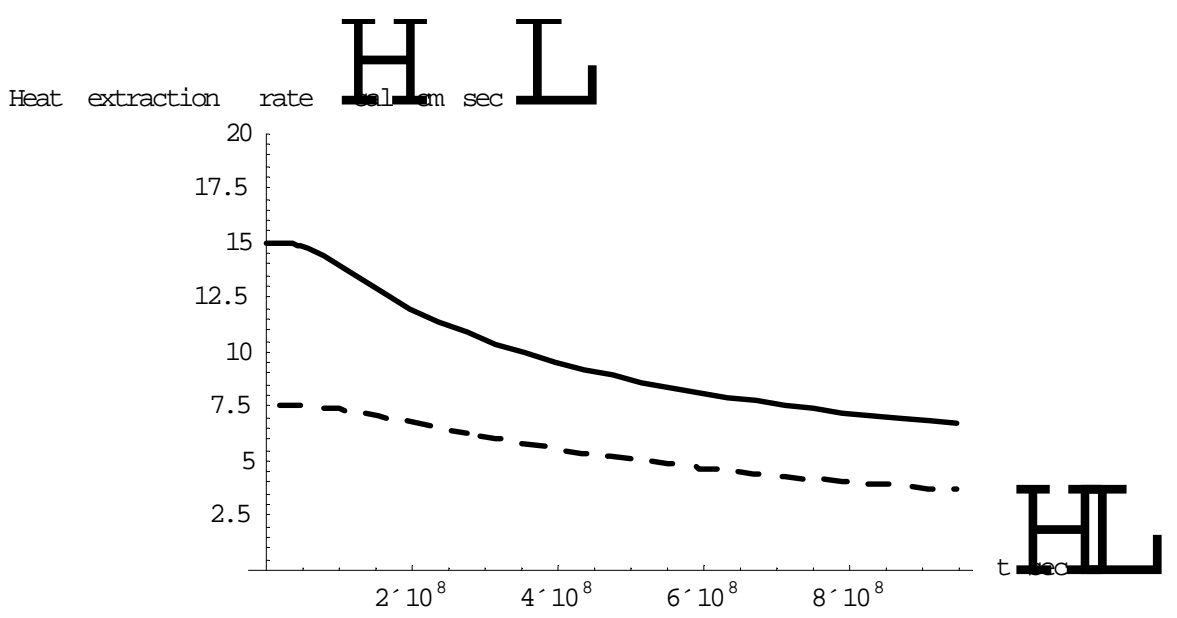

Figure 77: Heat extraction rate per unit height of fracture (dash line: 50\% leakoff; solid line: no leakoff) 


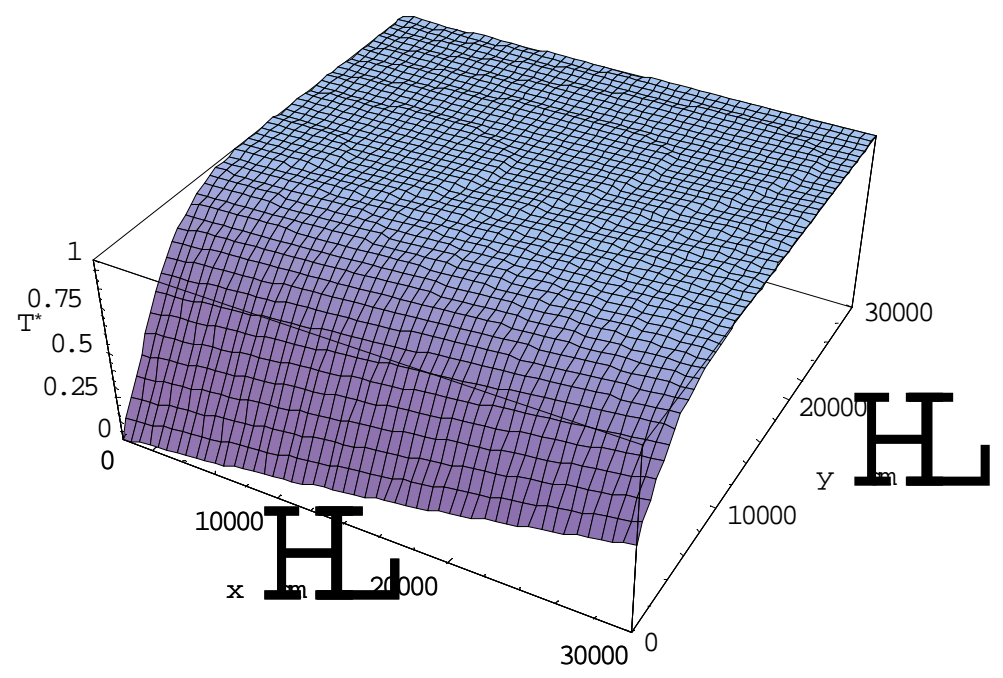

Figure 78: Temperature distribution in the reservoir and the fracture $(y=0)$ at $t=30 \mathrm{yr} ., 50 \%$ leakoff.

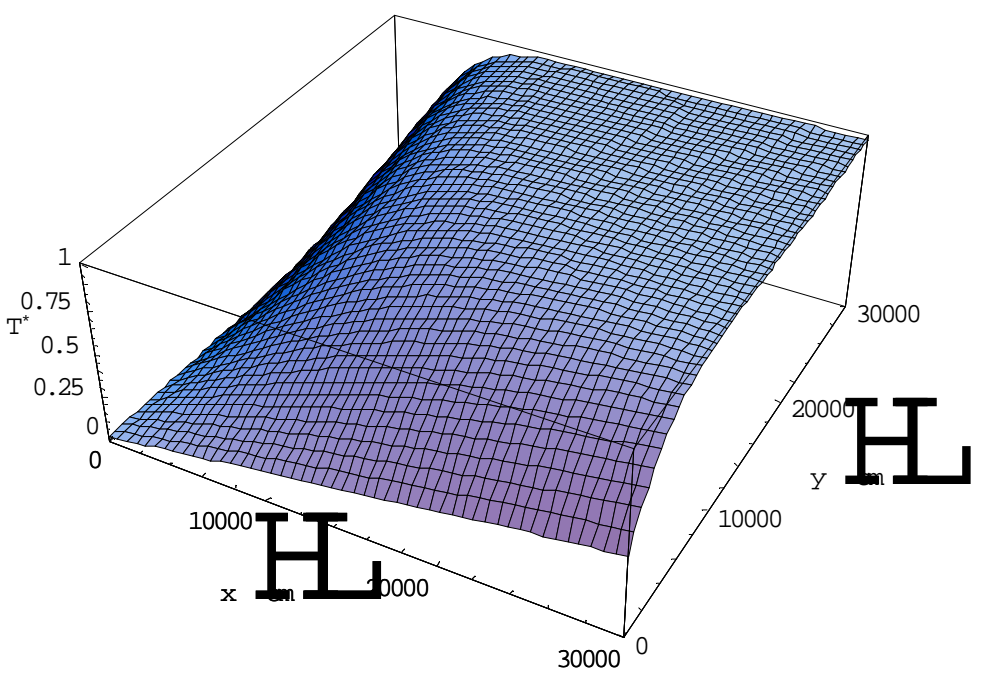

Figure 79: Temperature distribution in the reservoir and the fracture $(y=0)$ at $t=30$ yr., no leakoff. 


\subsubsection{Integral formulation \& solution of the $2 \mathrm{D}$ model}

The fist step in solution strategy for the 2D model involves eliminating the time variable by applying the Laplace transform to (33) and (34):

$$
\begin{aligned}
\frac{d \widetilde{T}_{w d}}{d x} & =\left.\frac{K_{r}}{v b \rho_{w} c_{w}} \frac{\partial \widetilde{T}_{r d}}{\partial y}\right|_{y=0^{+}} \\
\frac{\partial^{2} \widetilde{T}_{r d}}{\partial x^{2}}+\frac{\partial^{2} \widetilde{T}_{r d}}{\partial y^{2}} & =\frac{\rho_{r} c_{r} s}{K_{r}} \widetilde{T}_{r d}
\end{aligned}
$$

where the tilde denotes Laplace transform, and $s$ is the Laplace transform parameter. Note that in arriving at (136) and (136), the initial conditions (36) and (37) have incorporated. The boundary conditions (38) and (39) become:

$$
\begin{aligned}
\widetilde{T}_{w d}(0, s) & =\frac{1}{s} \\
\widetilde{T}_{r d}(x, 0, s) & =\widetilde{T}_{w d}(x, s)
\end{aligned}
$$

After solving the system, it is necessary to perform Laplace inversion to restore the time variable. This is handled by an approximate inversion algorithm known as the Stehfest method [82]; given data on the Laplace transformed function $F(s)$, the function in the time domain $f(t)$ can be found from the approximate formula:

$$
f(t) \approx \frac{\ln 2}{t} \sum_{n=1}^{N} c_{n} F\left(\frac{n \ln 2}{t}\right)
$$

where $N$ is the number of terms in the series ( must be even) and the coefficient $c_{n}$ is given by

$$
c_{n}=(-1)^{n+\frac{N}{2}} \sum_{k=\frac{n+1}{2}}^{\min \left(n, \frac{N}{2}\right)} \frac{k^{\frac{N}{2}}(2 k) !}{\left(\frac{N}{2}-k\right) ! k !(k-1) !(n-k) !(2 k-n) !}
$$

Integral equation formulation The solution system, (136) to (138), is defined in two spatial dimensions. It shall now be demonstrated that by utilizing the Green's function method, one can convert the system into a one-dimensional integral equation, thus significantly reduce the solution effort.

The temperature in the reservoir due to a continuous point heat source of magnitude $Q_{T}$ is governed by the equation:

$$
K_{r}\left(\frac{\partial^{2} G}{\partial x^{2}}+\frac{\partial^{2} G}{\partial y^{2}}\right)-\rho_{r} c_{r} s G=-\frac{Q_{T}}{s} \delta\left(\mathbf{x}-\mathbf{x}^{\prime}\right)
$$

where $\delta$ is the Dirac delta function, and $\mathbf{x}^{\prime}$ is the source location. The solution of (141) is the well known Green's function:

$$
G=\frac{Q_{T}}{2 \pi K_{r} s} \mathrm{~K}_{0}\left(\sqrt{\frac{\rho_{r} c_{r} s}{K_{r}}} r\right)
$$

where $\mathrm{K}_{0}$ is the modified Bessel function of the second kind of order zero, and $r=\left\|\mathbf{x}-\mathbf{x}^{\prime}\right\|$ is the Euclidean distance between two points. The temperature in the reservoir due to a distribution of line source along the 
fracture trajectory is given by:

$$
\widetilde{T}_{r d}(x, y, s)=\frac{1}{2 \pi K_{r}} \int_{0}^{L} \widetilde{q}\left(x^{\prime}, s\right) \mathrm{K}_{0}\left(\sqrt{\frac{\rho_{r} c_{r} s}{K_{r}}} r\right) d x^{\prime}
$$

where $\widetilde{q}$ is the Laplace transform of line source intensity. The line source intensity can be represented by the heat flux:

$$
\widetilde{q}(x, s)=-\left.2 K_{r} \frac{\partial \widetilde{T}_{r d}}{\partial y}\right|_{y=0^{+}}=-2 v b \rho_{w} c_{w} \frac{d \widetilde{T}_{w d}}{d x}
$$

according to (136) and (138). Substituting (144) into (141) yields:

$$
\widetilde{T}_{r d}(x, y, s)=-\frac{v b \rho_{w} c_{w}}{\pi K_{r}} \int_{0}^{L} \frac{d \widetilde{T}_{w d}\left(x^{\prime}, s\right)}{d x^{\prime}} \mathrm{K}_{0}\left(\sqrt{\frac{\rho_{r} c_{r} s}{K_{r}}} r\right) d x^{\prime}
$$

Performing integration by parts, the above equation transforms into:

$$
\begin{aligned}
\frac{\pi K_{r}}{v b \rho_{w} c_{w}} \widetilde{T}_{r d}(x, y, s)= & -\widetilde{T}_{w d}(L, s) \mathrm{K}_{0}\left(\sqrt{\frac{\rho_{r} c_{r} s}{K_{r}}\left[(x-L)^{2}+y^{2}\right]}\right) \\
& +\frac{1}{s} \mathrm{~K}_{0}\left(\sqrt{\frac{\rho_{r} c_{r} s}{K_{r}}\left(x^{2}+y^{2}\right)}\right) \\
+ & \sqrt{\frac{\rho_{r} c_{r} s}{K_{r}}} \int_{0}^{L} \widetilde{T}_{w d}\left(x^{\prime}, s\right) \frac{x-x^{\prime}}{\sqrt{\left(x-x^{\prime}\right)^{2}+y^{2}}} \mathrm{~K}_{1}\left(\sqrt{\frac{\rho_{r} c_{r} s}{K_{r}}} r\right) d x^{\prime}
\end{aligned}
$$

In the above, the boundary condition (137) has been utilized. Applying the above equation along the fracture trajectory, $y=0$ and $0 \leq x \leq L$, and setting $\widetilde{T}_{r d}=\widetilde{T}_{w d}$ according to (138), yields:

$$
\begin{aligned}
\frac{\pi K_{r}}{v b \rho_{w} c_{w}} \widetilde{T}_{w d}(x, s)= & -\widetilde{T}_{w d}(L, s) \mathrm{K}_{0}\left(\sqrt{\frac{\rho_{r} c_{r} s}{K_{r}}}(L-x)\right) \\
& +\frac{1}{s} \mathrm{~K}_{0}\left(\sqrt{\frac{\rho_{r} c_{r} s}{K_{r}}} x\right) \\
& +\sqrt{\frac{\rho_{r} c_{r} s}{K_{r}}} \int_{0}^{L} \widetilde{T}_{w d}\left(x^{\prime}, s\right) \frac{x-x^{\prime}}{\left|x-x^{\prime}\right|} \mathrm{K}_{1}\left(\sqrt{\frac{\rho_{r} c_{r} s}{K_{r}}}\left|x-x^{\prime}\right|\right) d x^{\prime} \\
\text { for } 0 \leq & x \leq L
\end{aligned}
$$

The above integral equation has incorporated all the initial and boundary conditions and has eliminated the reservoir temperature as a variable. It is a sufficient solution system to solved for $\widetilde{T}_{w d}$ in the one-dimensional space.

Numerical strategy Equation (147) is a Fredholm integral equation of the second kind [83]. It is Cauchy singular due to the $1 / r$ singularity contained in the Bessel function $\mathrm{K}_{1}$, which needs to be regularized before a numerical integration can be performed. For the purpose of regularization, first replace $\widetilde{T}_{w d}\left(x^{\prime}, s\right)$ in the singular integral in (147) by $\widetilde{T}_{w d}(x, s)$ and perform the integration analytically:

$$
\sqrt{\frac{\rho_{r} c_{r} s}{K_{r}}} \int_{0}^{L} \widetilde{T}_{w d}(x, s) \frac{x-x^{\prime}}{\left|x-x^{\prime}\right|} \mathrm{K}_{1}\left(\sqrt{\frac{\rho_{r} c_{r} s}{K_{r}}}\left|x-x^{\prime}\right|\right) d x^{\prime}=
$$




$$
\widetilde{T}_{w d}(x, s)\left[\mathrm{K}_{0}\left(\sqrt{\frac{\rho_{r} c_{r} s}{K_{r}}}(L-x)\right)-\mathrm{K}_{0}\left(\sqrt{\frac{\rho_{r} c_{r} s}{K_{r}}} x\right)\right]
$$

Then (148) can be subtracted from (147) to give:

$$
\begin{gathered}
{\left[\frac{\pi K_{r}}{v b \rho_{w} c_{w}}-\mathrm{K}_{0}\left(\sqrt{\frac{\rho_{r} c_{r} s}{K_{r}}}(L-x)\right)+\mathrm{K}_{0}\left(\sqrt{\frac{\rho_{r} c_{r} s}{K_{r}}} x\right)\right] \widetilde{T}_{w d}(x, s)=} \\
+\sqrt{\frac{\rho_{r} c_{r} s}{K_{r}}} \int_{0}^{L}\left[\widetilde{T}_{w d}\left(x^{\prime}, s\right)-\widetilde{T}_{w d}(x, s)\right] \frac{x-x^{\prime}}{\left|x-x^{\prime}\right|} \mathrm{K}_{1}\left(\sqrt{\frac{\rho_{r} c_{r} s}{K_{r}}}\left|x-x^{\prime}\right|\right) d x^{\prime} \\
-\widetilde{T}_{w d}(L, s) \mathrm{K}_{0}\left(\sqrt{\frac{\rho_{r} c_{r} s}{K_{r}}}(L-x)\right)+\frac{1}{s} \mathrm{~K}_{0}\left(\sqrt{\frac{\rho_{r} c_{r} s}{K_{r}}} x\right) \\
\text { for } 0 \leq x \leq L
\end{gathered}
$$

We note that the integral is no longer singular; hence the equation is regularized. To approximately solve (149), a numerical quadrature rule is applied over it. Because it is important to include the end points, $x=0, L$, in the formula, the Simpson's rule [84] is applied rather than the Gaussian quadrature formula. Hence (149) becomes:

$$
\begin{gathered}
{\left[\frac{\pi K_{r}}{v b \rho_{w} c_{w}}-\mathrm{K}_{0}\left(\sqrt{\frac{\rho_{r} c_{r} s}{K_{r}}}\left(L-x_{i}\right)\right)+\mathrm{K}_{0}\left(\sqrt{\frac{\rho_{r} c_{r} s}{K_{r}}} x_{i}\right)\right] T_{i}=} \\
-T_{n} \mathrm{~K}_{0}\left(\sqrt{\frac{\rho_{r} c_{r} s}{K_{r}}}\left(L-x_{i}\right)\right)+T_{0} \mathrm{~K}_{0}\left(\sqrt{\frac{\rho_{r} c_{r} s}{K_{r}}} x_{i}\right) \\
+\sqrt{\frac{\rho_{r} c_{r} s}{K_{r}}} \frac{L}{n} \sum_{j=0}^{n} w_{j}\left(T_{j}-T_{i}\right) \frac{x_{i}-x_{j}}{\left|x_{i}-x_{j}\right|} \mathrm{K}_{1}\left(\sqrt{\frac{\rho_{r} c_{r} s}{K_{r}}}\left|x_{i}-x_{j}\right|\right) \\
\text { for } i=1, \ldots, n
\end{gathered}
$$

where $n$ is the number of equal intervals, $x_{i}=i L / n$ for $i=0, \ldots, n$ are the abscissas, $w_{i}$ are weights, and $T_{i}$ denotes $\widetilde{T}_{w d}\left(x_{i}, s\right)$. For Simpson's $\frac{3}{8}$-rule, the weights are:

$$
w_{j}=\frac{3}{8}, \frac{7}{6}, \frac{23}{24}, 1,1, \ldots, 1,1, \frac{23}{24}, \frac{7}{6}, \frac{3}{8} ; \quad \text { for } j=0, \ldots, n
$$

Note that the system defined in (150) has $n+1$ discrete values of $T_{i}$. The first value $T_{0}$ is given by the boundary condition $T_{0}=1 / \mathrm{s}$. Hence there exist $n$ unknowns and $n$ equations for $i=1, \ldots, n$. This forms a linear system

$$
[\mathbf{A}]\{\mathbf{T}\}=\{\mathbf{b}\}
$$

which can be solved for $T_{i}$. A Fortran computer program for has been developed for solving (150). 
Example The effect of two-dimensional versus one-dimensional heat conduction is examined using a numerical example below. For this case, we choose the following data set

$$
L=300 \mathrm{~m} ; \quad b=0.3 \mathrm{~cm} ; \quad v=0.5 \mathrm{~cm} / \mathrm{s}
$$

Other parameters remain the same. Figure 80 presents the temperature profile along the fracture length at three different times, 1,5 , and 15 years after production. Due to the rapidly varying function in a wide range of Laplace transform parameters, 200 nodes are needed to have an accurate quadrature by Simpson's rule and numerical Laplace inversion by Stehfest method. The temperature shown is the normalized temperature $T_{w}=\left(T-T_{w o}\right) /\left(T_{r o}-T_{w o}\right)=1-T_{D}$. The two-dimensional heat conduction solution (solid lines) is computed based on (163), while the one-dimensional solution (dash line) is given by the Lowell solution (83). We observe that the 2-D case always predicts a higher temperature for the obvious reason that more heat is supplied from the additional hot rock region. The increase in temperature is more significant near the inlet than outlet, and at large than small time.

The most useful information for the application is the extraction temperature. Figure 81 shows a plot of the normalized extraction temperature $T_{w}(L, 0, t)$ for 30 years of production time. It can be observed that the 2-D heat conduction case always predicts a higher extraction temperature. The difference is more pronounced at large production time. At the end of 30 years, the predicted temperature $T_{w}$ of the 2-D case is about $10 \%$ higher than the 1-D case. Another way to envision the difference is that the 1-D case predicts that the heat extraction efficiency drops to below $50 \%$ after 23 years of production, while the 2-D case predicts 29 years.

Kolditz has reported that the production temperature predicted by the three-dimensional model was significantly higher than that of the one-dimensional model. Specifically, after 20 years production temperatures of $67 \%$ and $56 \%$ of initial reservoir temperature were reported for the two cases, respectively. Figure 82 shows the normalized temperature of water form a fracture in hot dry rock using the two-dimensional boundary element model. The one-D solution of Lowell is also shown. Note that the difference between $1 \mathrm{D}$ and 2D solutions is insignificant only for longer fractures, given the same injection rate. This is to be expected as the rate of heat extraction from the rock decreases. Thus, it takes a much longer time for the two-dimensional diffusion mechanism to develop in the rock mass. This explains the large differences between the 3D and 1D heat extraction from a circular fracture reported by the Kolditz (1995). Such differences seem to occur only under high water injection rates.

Calculation of thermally-induced stress field It is of interest to calculate the tractions that are induced along the fracture(s) by the perturbation of the temperature field due to extraction of heat. The induced thermal stress can explain the injection induced seismicity observed by a number of investigators [135], [136]. The latter has found that half the earthquakes in The Geysers field seem to be associated with cold water injection. The mechanism by which seismicity occurs is well understood and is based on the Coulomb criterion, $\tau=\left(\sigma_{n}-p\right)+C$; a reduction in effective stress acting across the fracture drives it to slip in shear.

In this work a two-dimensional constant element real displacement discontinuity model [99] is used to treat the problem. The thermal stresses are calculated from the source strengths obtained from the 2D boundary element solution of heat extraction. Figure 83 illustrates the thermal stresses for the heat extraction problem treated above (using a $\Delta T=380{ }^{\circ} \mathrm{C}$ ). Note that significant stresses are generated on the facture 


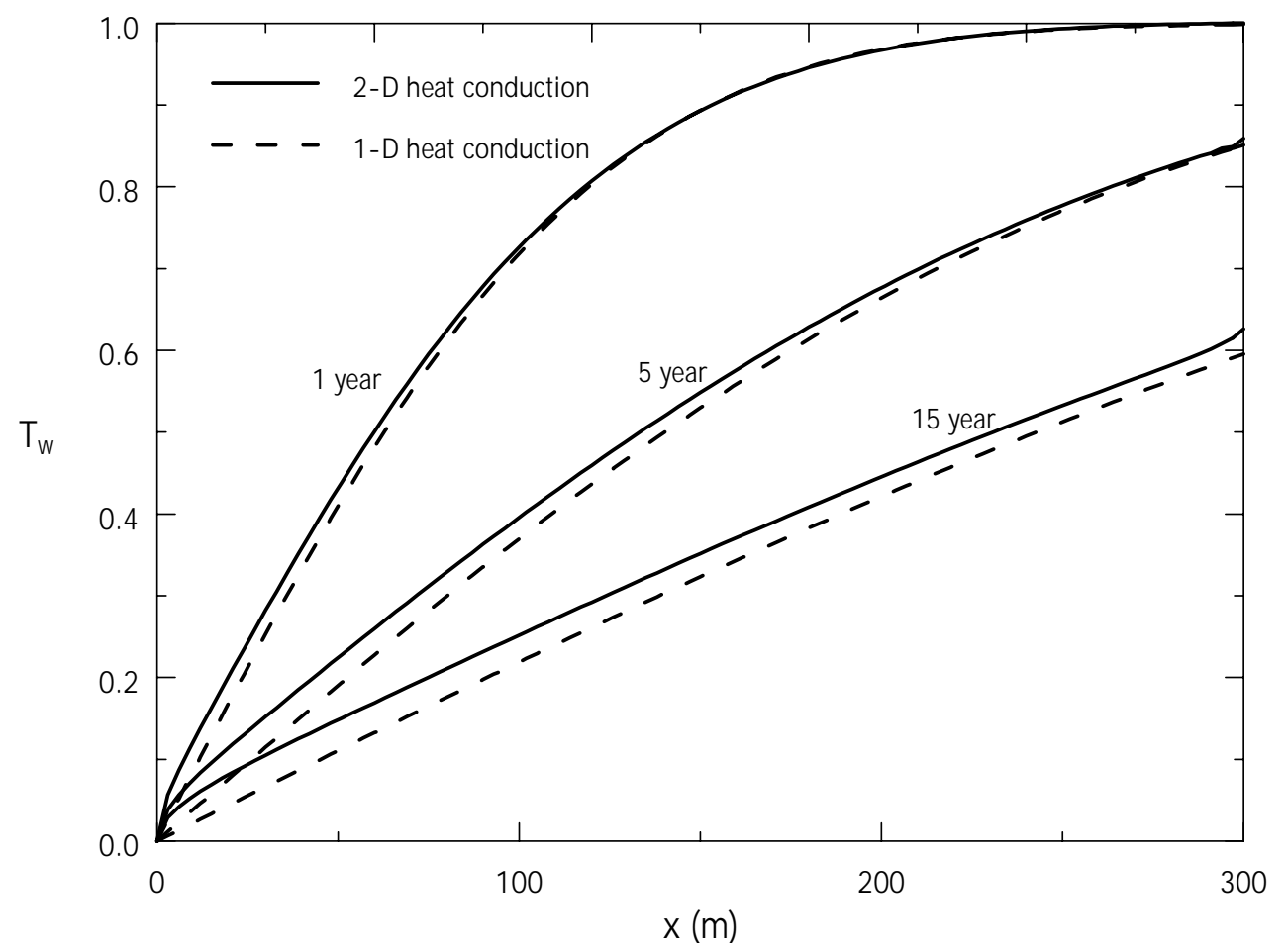

Figure 80: Normalized temperature distribution in the fracture based on 1-D and 2-D heat conduction models.

face (increasing with time). This suggests that when compared to pore pressure, the thermal stresses play a more significant role in joint and fault slip and associated seismicity, as observed by [100] for an injection well. The magnitude of the thermal stresses associated with advective cooling was estimated analytically by [100] using an axisymmetric model of injection into a planar reservoir and a $1 \mathrm{D}$ heat flow in the rock mass. According to their analysis, for time periods on the order of a few years, the thermal stress perturbation is proportional to $\left(\frac{1}{3} \Delta T G \beta_{s}\left[c^{*} t\right]^{\frac{1}{4}}\right) m^{\frac{-1}{2}}$; where $m$ is the mass injection rate.

Coupling fluid flow, thermal, and mechanical effects The two-dimensional injection/operation scheme has been coupled to the mechanical fracture propagation model. The corresponding numerical algorithms and the program allow one to calculate slip on the fractures due to thermally-induced variations of in-situ stress. One can also study the impact of long term injection on fracture propagation. Due to time limitations, however, examples are not presented at this time. The modifications of the interface to allow consideration of such problems along with some numerical examples will be accomplished in the next phase of work.

The aforementioned thermoelastic work is based on the assumption of rock homogeneity. The presence of inhomogeneities has recently been addressed [137] by developing a steady-state CV-BEM thermoelastic model. Its extension to include transient effects is forthcoming. 


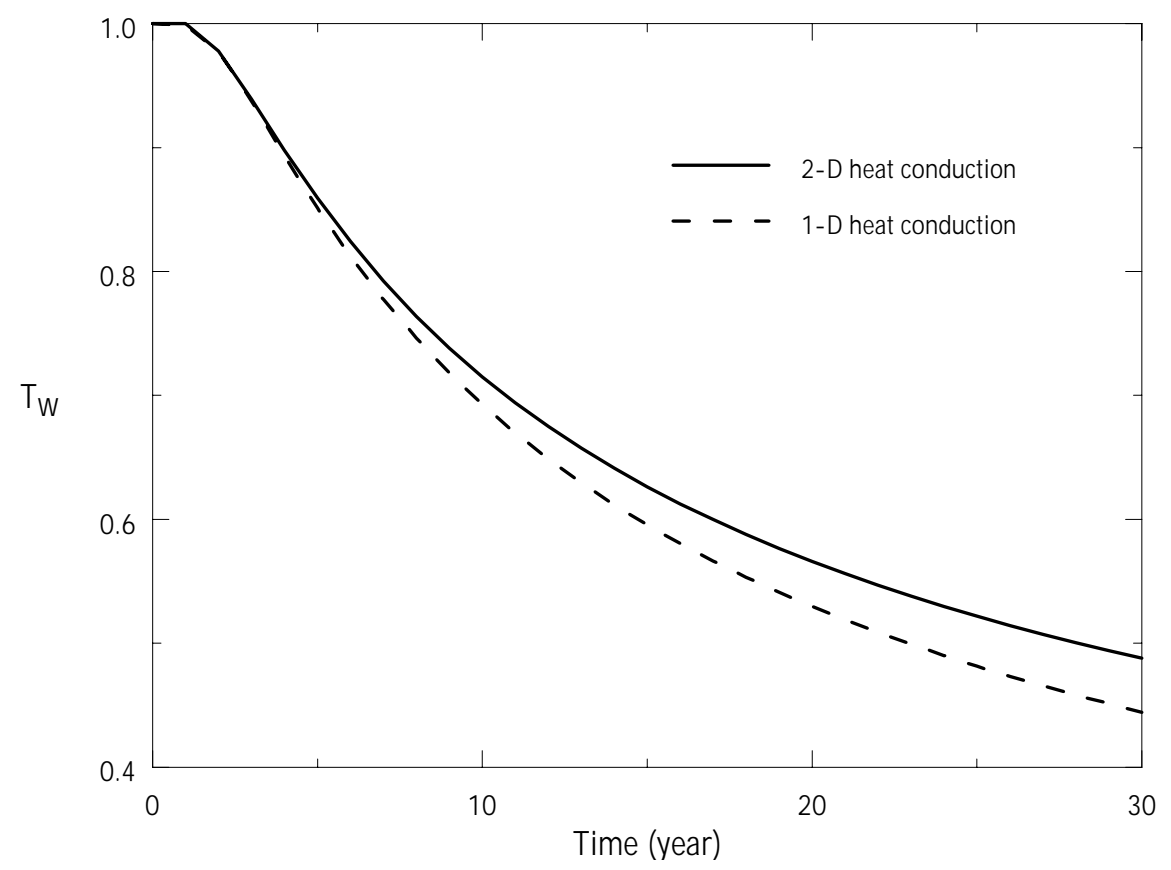

Figure 81: Normalized extraction temperature based on 1-D and 2-D heat conduction models.

\subsubsection{Integral formulation \& solution for the 3D model}

Normalized solution system As before, it is desirable to normalize the solution field with a value between zero and one. Therefore, we introduce a normalized temperature deficit by:

$$
T_{d}=\frac{T_{o}-T}{T_{o}-T_{i n j}}
$$

Equations (46) and (48) retain their original forms under this new variable,

$$
\begin{gathered}
K_{r} \nabla_{3}^{2} T_{d}(x, y, z, t)=\rho_{r} c_{r} \frac{\partial T_{d}(x, y, z, t)}{\partial t} \\
\rho_{w} c_{w} \mathbf{q}(x, y) \cdot \nabla_{2} T_{d}(x, y, 0, t)-\left.2 K_{r} \frac{\partial T_{d}(x, y, z, t)}{\partial z}\right|_{z=0^{+}}=0
\end{gathered}
$$

The initial condition is then:

$$
T_{d}(x, y, z, 0)=0
$$

and the boundary condition becomes:

$$
T_{d}\left(x_{i}, y_{i}, 0, t\right)=1
$$

To facilitate the treatment of the time variable, we apply Laplace transform to the above equations and obtain:

$$
K_{r} \nabla_{3}^{2} \widetilde{T}_{d}(x, y, z, s)=s \rho_{r} c_{r} \widetilde{T}_{d}(x, y, z, s)
$$




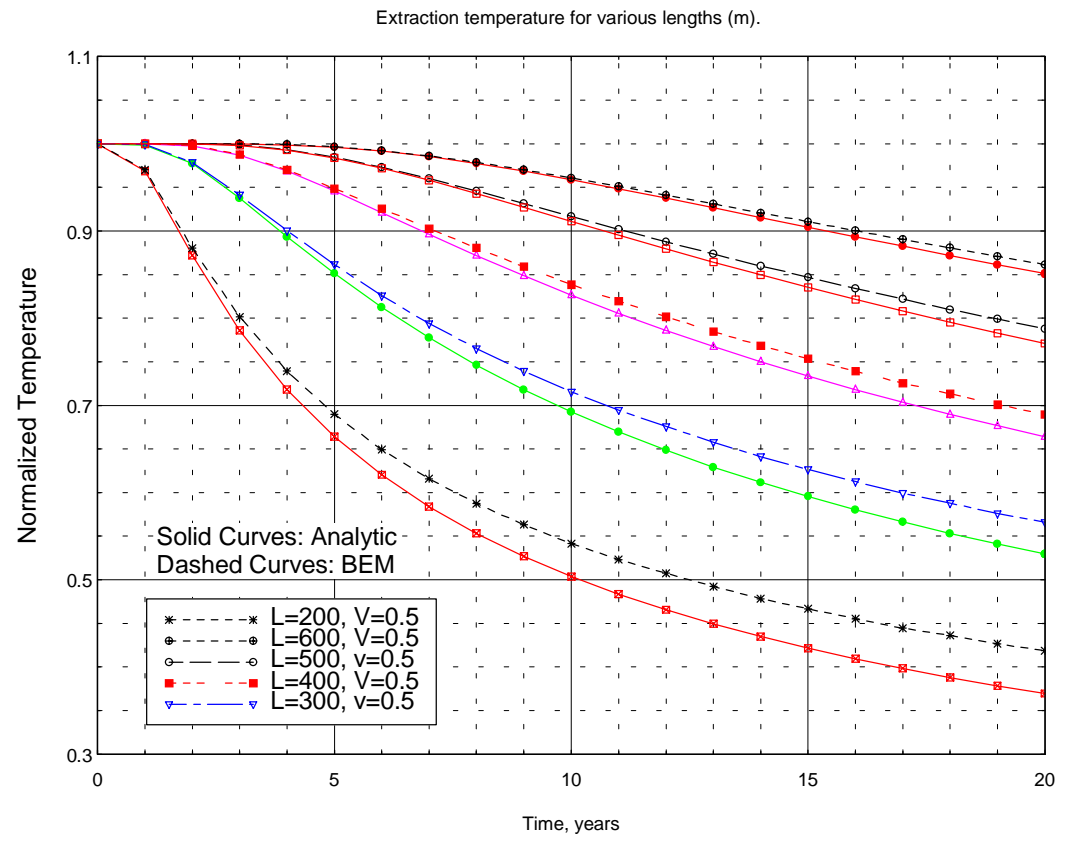

Figure 82: Normalized water temperature extracted form a fracture using the 2D BEM (fluid velocity of 0.5 $\mathrm{cm} / \mathrm{sec}$, see Cheng et al., 2001 for fluid \& rock properties). 


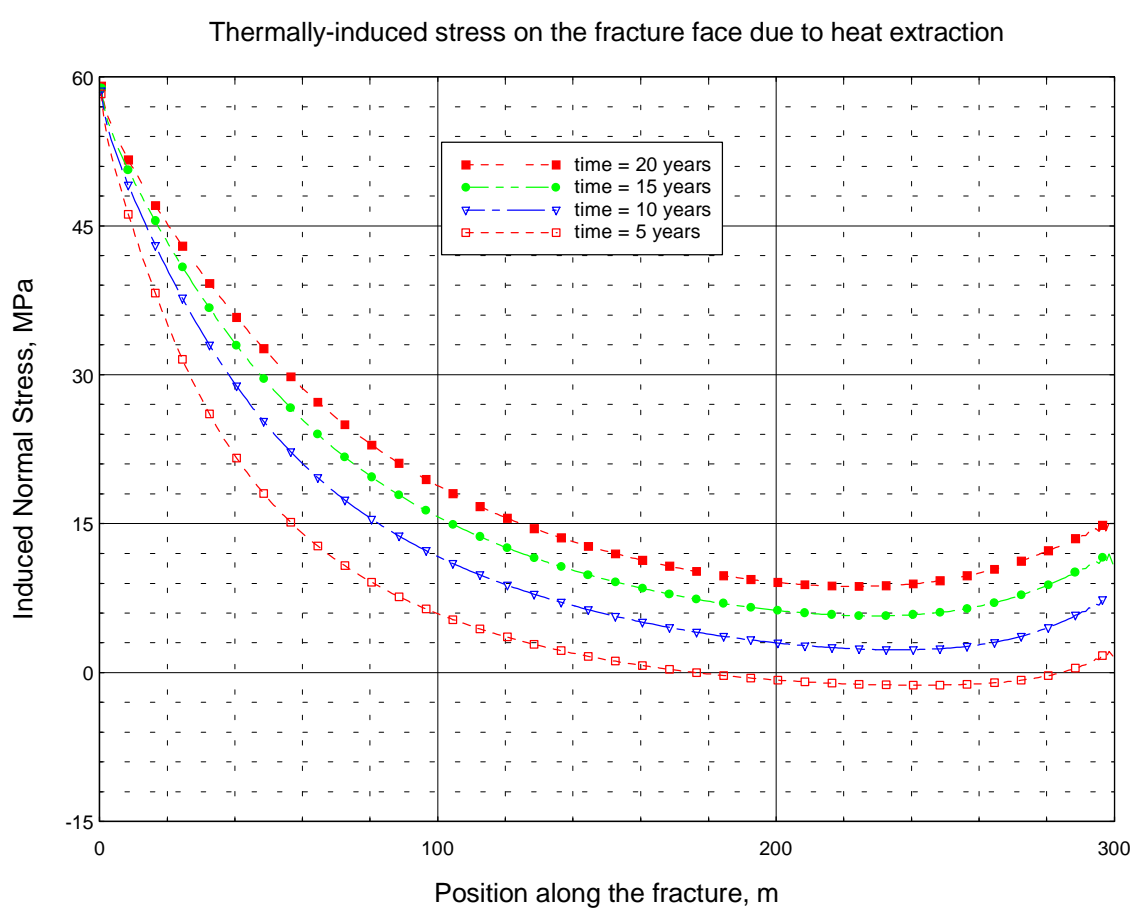

Figure 83: Thermal stresses on the fracture surface in an injection/extraction operation. 


$$
\begin{gathered}
\rho_{w} c_{w} \mathbf{q}(x, y) \cdot \nabla_{2} \widetilde{T}_{d}(x, y, 0, s)-\left.2 K_{r} \frac{\partial \widetilde{T}_{d}(x, y, z, s)}{\partial z}\right|_{z=0^{+}}=0 \\
\widetilde{T}_{d}\left(x_{i}, y_{i}, 0, s\right)=\frac{1}{s}
\end{gathered}
$$

where the wiggle overbar denotes the Laplace transform, and $s$ is the transform parameter. We note that the initial condition (156) has been absorbed into (158). hence, Equations (158)-(160) form a complete solution system.

For convenience, we also introduce the alternative form of (159) based on (47), as follows:

$$
\begin{array}{r}
\rho_{w} c_{w} \nabla_{2} \cdot\left[\mathbf{q}(x, y) \widetilde{T}_{d}(x, y, 0, s)\right]-\left.2 K_{r} \frac{\partial \widetilde{T}_{d}(x, y, z, s)}{\partial z}\right|_{z=0^{+}}= \\
\rho_{w} c_{w} Q\left[\widetilde{T}_{d}\left(x_{e}, y_{e}, 0, s\right) \delta\left(x-x_{e}, y-y_{e}\right)-\frac{1}{s} \delta\left(x-x_{i}, y-y_{i}\right)\right]
\end{array}
$$

Integral equation formulation The system of equations (47)-(48) is defined in three spatial dimensions. We shall demonstrate that by utilizing Green's function of three-dimensional diffusion equation, the solution system can be reduced to a two-dimensional integral equation. The numerical discretization is performed on the fracture surface only, significantly reducing the computational cost.

The Laplace transform of temperature deficit in the reservoir due to a continuous point heat source of unit magnitude and located at $\left(x^{\prime}, y^{\prime}, z^{\prime}\right)$ is given by the fundamental solution of the modified Helmholtz equation (158) as:

$$
\widetilde{G}=\frac{1}{4 \pi K_{r}} \frac{1}{R} \exp \left(-\sqrt{\frac{\rho_{r} c_{r} s}{K_{r}}} R\right)
$$

where

$$
R=\sqrt{\left(x-x^{\prime}\right)^{2}+\left(y-y^{\prime}\right)^{2}+\left(z-z^{\prime}\right)^{2}}
$$

is the Euclidean distance. The temperature in the reservoir due to a distribution of sources on the fracture surface $A$ is then written as:

$$
\widetilde{T}_{d}(x, y, z, s)=\frac{1}{4 \pi K_{r}} \int_{A} \widetilde{\mu}\left(x^{\prime}, y^{\prime}, s\right) \frac{1}{R_{1}} \exp \left(-\sqrt{\frac{\rho_{r} c_{r} s}{K_{r}}} R_{1}\right) d x^{\prime} d y^{\prime}
$$

where $\widetilde{\mu}$ is the source intensity, and

$$
R_{1}=\sqrt{\left(x-x^{\prime}\right)^{2}+\left(y-y^{\prime}\right)^{2}+z^{2}}
$$

We realize that the heat lost in the reservoir is just the heat gained by the fracture fluid through the wall; hence the source strength is just

$$
\widetilde{\mu}(x, y, s)=-\left.2 K_{r} \frac{\partial \widetilde{T}_{d}(x, y, z, s)}{\partial z}\right|_{z=0^{+}}
$$

According to (159), we can tie the heat source to the heat conservation in the fracture as

$$
\widetilde{\mu}(x, y, s)=-\rho_{w} c_{w} \mathbf{q}(x, y) \cdot \nabla_{2} \widetilde{T}_{d}(x, y, 0, s)
$$


Substituting (167) into (164), we obtain:

$$
\widetilde{T}_{d}(x, y, z, s)=\frac{-\rho_{w} c_{w}}{4 \pi K_{r}} \int_{A}\left[\mathbf{q}\left(x^{\prime}, y^{\prime}\right) \cdot \nabla_{2} \widetilde{T}_{d}\left(x^{\prime}, y^{\prime}, 0, s\right)\right] \frac{1}{R_{1}} \exp \left(-\sqrt{\frac{\rho_{r} c_{r} s}{K_{r}}} R_{1}\right) d x^{\prime} d y^{\prime}
$$

Equation (168) is the integral equation representation of temperature at any point $(x, y, z)$ in the reservoir, provided that the temperature on the fracture surface $\widetilde{T}_{d}(x, y, 0, s)$ is known.

To solve the temperature on the fracture surface, we restrict (168) to only points on the fracture surface $A$, and it becomes

$$
\begin{aligned}
& \widetilde{T}_{d}(x, y, 0, s)=\frac{-\rho_{w} c_{w}}{4 \pi K_{r}} \int_{A}\left[\mathbf{q}\left(x^{\prime}, y^{\prime}\right) \cdot \nabla_{2} \widetilde{T}_{d}\left(x^{\prime}, y^{\prime}, 0, s\right)\right] \frac{1}{r} \exp \left(-\sqrt{\frac{\rho_{r} c_{r} s}{K_{r}}} r\right) d x^{\prime} d y^{\prime} ; \\
& x, y \in A
\end{aligned}
$$

where

$$
r=\sqrt{\left(x-x^{\prime}\right)^{2}+\left(y-y^{\prime}\right)^{2}}
$$

Equation (169) is entirely defined on the two-dimensional plane $A$. Together with the boundary condition (160), it forms a complete solution system for fluid temperature in the fracture.

Equation (169) contains the temperature gradient as an unknown, which requires a finite difference approximation in numerical solution. Sometimes it is more desirable to directly model temperature for better accuracy. An alternative formula of (169) can be derived as follows. Utilizing (161) instead of (159) in (167) and (168), we obtain

$$
\begin{gathered}
\widetilde{T}_{d}(x, y, z, s)=\frac{-\rho_{w} c_{w}}{4 \pi K_{r}} \int_{A} \nabla_{2} \cdot\left[\widetilde{T}_{d}\left(x^{\prime}, y^{\prime}, 0, s\right) \mathbf{q}\left(x^{\prime}, y^{\prime}\right)\right] \frac{1}{R_{1}} \exp \left(-\sqrt{\frac{\rho_{r} c_{r} s}{K_{r}}} R_{1}\right) d x^{\prime} d y^{\prime} \\
+\frac{\rho_{w} c_{w} Q}{4 \pi K_{r}}\left[\widetilde{T}_{d}\left(x_{e}, y_{e}, 0, s\right) \frac{1}{R_{e}} \exp \left(-\sqrt{\frac{\rho_{r} c_{r} s}{K_{r}}} R_{e}\right)-\frac{1}{s} \frac{1}{R_{i}} \exp \left(-\sqrt{\frac{\rho_{r} c_{r} s}{K_{r}}} R_{i}\right)\right]
\end{gathered}
$$

where

$$
\begin{aligned}
R_{e} & =\sqrt{\left(x-x_{e}\right)^{2}+\left(y-y_{e}\right)^{2}+z^{2}} \\
R_{i} & =\sqrt{\left(x-x_{i}\right)^{2}+\left(y-y_{i}\right)^{2}+z^{2}}
\end{aligned}
$$

The divergence operator in (171) can be relaxed by the divergence theorem

$$
\begin{gathered}
\int_{A} \nabla_{2} \cdot\left[\widetilde{T}_{d}\left(x^{\prime}, y^{\prime}, 0, s\right) \mathbf{q}\left(x^{\prime}, y^{\prime}\right)\right] \frac{1}{R_{1}} \exp \left(-\sqrt{\frac{\rho_{r} c_{r} s}{K_{r}}} R_{1}\right) d x^{\prime} d y^{\prime} \\
=\int_{A} \nabla_{2} \cdot\left[\widetilde{T}_{d}\left(x^{\prime}, y^{\prime}, 0, s\right) \mathbf{q}\left(x^{\prime}, y^{\prime}\right) \frac{1}{R_{1}} \exp \left(-\sqrt{\frac{\rho_{r} c_{r} s}{K_{r}}} R_{1}\right)\right] d x^{\prime} d y^{\prime} \\
\quad-\int_{A} \widetilde{T}_{d}\left(x^{\prime}, y^{\prime}, 0, s\right) \mathbf{q}\left(x^{\prime}, y^{\prime}\right) \cdot \nabla_{2}\left[\frac{1}{R_{1}} \exp \left(-\sqrt{\frac{\rho_{r} c_{r} s}{K_{r}}} R_{1}\right)\right] d x^{\prime} d y^{\prime} \\
=\int_{\partial A} \widetilde{T}_{d}\left(x^{\prime}, y^{\prime}, 0, s\right) q_{n}\left(x^{\prime}, y^{\prime}\right) \frac{1}{R_{1}} \exp \left(-\sqrt{\frac{\rho_{r} c_{r} s}{K_{r}}} R_{1}\right) d x^{\prime} d y^{\prime} \\
\quad-\int_{A} \widetilde{T}_{d}\left(x^{\prime}, y^{\prime}, 0, s\right) \mathbf{q}\left(x^{\prime}, y^{\prime}\right) \cdot \nabla_{2}\left[\frac{1}{R_{1}} \exp \left(-\sqrt{\frac{\rho_{r} c_{r} s}{K_{r}}} R_{1}\right)\right] d x^{\prime} d y^{\prime}
\end{gathered}
$$


where $q_{n}$ is the normal component of $\mathbf{q}$ on $\partial A$. We further note that in (173) the line integral performed over the fracture boundary $\partial A$ vanishes by the virtue of the no flux condition $q_{n}=0$ as indicated in (45). Substituting (173) into (171) and applying the equation to the fracture surface only, we obtain the alternative to the solution system (169):

$$
\begin{gathered}
\widetilde{T}_{d}(x, y, 0, s)=\frac{\rho_{w} c_{w}}{4 \pi K_{r}} \int_{A} \widetilde{T}_{d}\left(x^{\prime}, y^{\prime}, 0, s\right) \mathbf{q}\left(x^{\prime}, y^{\prime}\right) \cdot \nabla_{2}\left[\frac{1}{r} \exp \left(-\sqrt{\frac{\rho_{r} c_{r} s}{K_{r}}} r\right)\right] d x^{\prime} d y^{\prime} \\
+\frac{\rho_{w} c_{w} Q}{4 \pi K_{r}}\left[\widetilde{T}_{d}\left(x_{e}, y_{e}, 0, s\right) \frac{1}{r_{e}} \exp \left(-\sqrt{\frac{\rho_{r} c_{r} s}{K_{r}}} r_{e}\right)-\frac{1}{s} \frac{1}{r_{i}} \exp \left(-\sqrt{\frac{\rho_{r} c_{r} s}{K_{r}}} r_{i}\right)\right]
\end{gathered}
$$

where

$$
\begin{aligned}
r_{e} & =\sqrt{\left(x-x_{e}\right)^{2}+\left(y-y_{e}\right)^{2}} \\
r_{i} & =\sqrt{\left(x-x_{i}\right)^{2}+\left(y-y_{i}\right)^{2}}
\end{aligned}
$$

We note that in this formulation the gradient operator is transferred to the known fundamental solution, which can be exactly executed.

Numerical implementation The general scheme for solving the system represented by (169) or (174) involves discretizing an arbitrary shaped fracture surface into a number of elements defined by a total of $n+1$ nodes. An unknown temperature deficit $\widetilde{T}_{d}^{i}$ is assigned to each node, with the exception of the injection point, where $\widetilde{T}_{d}=1 / s$ is the imposed boundary condition. As a result, there are $n$ unknown discrete temperatures. Equation (169) or (174) is then applied to the $n$ nodes by selecting in turn their nodal locations as the base point. This produces $n$ equations to be solved for the $n$ unknowns. Some detail of the numerical implementation is given below.

In the present work, we choose formulation (169) instead of (174) for the convenience of implementing an upwind scheme similar to the finite difference method. First, we subdivide the domain $A$ into $n_{e}$ rectangular elements using a finite difference mesh as shown in Figure 84. The area integration in (169) is performed element by element with reference to local coordinates $(\eta, \xi)$ (see Figure 84 ):

$$
\begin{aligned}
\widetilde{T}_{d}(x, y, s)=\frac{-\rho_{w} c_{w}}{4 \pi K_{r}} \sum_{m=1}^{n_{e}} \int_{A_{m}} & {\left[q_{x}(\eta, \xi) \frac{\partial \widetilde{T}_{d}(\eta, \xi, s)}{\partial \eta}+q_{y}(\eta, \xi) \frac{\partial \widetilde{T}_{d}(\eta, \xi, s)}{\partial \xi}\right] } \\
& \frac{1}{r} \exp \left(-\sqrt{\frac{\rho_{r} c_{r} s}{K_{r}}} r\right) d \eta d \xi
\end{aligned}
$$

The values of $q_{x}, q_{y}, \partial \widetilde{T}_{d} / \partial \eta$, and $\partial \widetilde{T}_{d} / \partial \xi$ within the element are interpolated from their nodal values based on bilinear shape functions; for example

$$
\frac{\partial \widetilde{T}_{d}}{\partial \eta}=\left(\frac{\partial \widetilde{T}_{d}}{\partial \eta}\right)_{1} N_{1}+\left(\frac{\partial \widetilde{T}_{d}}{\partial \eta}\right)_{2} N_{2}+\left(\frac{\partial \widetilde{T}_{d}}{\partial \eta}\right)_{3} N_{3}+\left(\frac{\partial \widetilde{T}_{d}}{\partial \eta}\right)_{4} N_{4}
$$


where the subscripts ()$_{i}$ denote the discrete nodal values, and

$$
\begin{aligned}
& N_{1}=\frac{1}{4}(1-\eta)(1-\xi) \\
& N_{2}=\frac{1}{4}(1+\eta)(1-\xi) \\
& N_{3}=\frac{1}{4}(1+\eta)(1+\xi) \\
& N_{4}=\frac{1}{4}(1-\eta)(1+\xi)
\end{aligned}
$$

are the shape functions. Values of $q_{x}$ and $q_{y}$ are directly specified on the nodes; $\partial \widetilde{T}_{d} / \partial \eta$ and $\partial \widetilde{T}_{d} / \partial \xi$, on the other hand, need to be approximated using finite difference formula based on nodal temperature values. Due to the presence of strong advection, an upwind differencing scheme is needed to maintain solution stability. At a grid point $(i, j)$ (see Figure 84), the temperature derivatives are expressed as backward or the forward differences according to the direction of the flow:

$$
\begin{aligned}
& \left(\frac{\partial \widetilde{T}_{d}}{\partial \eta}\right)_{i, j}=\frac{\widetilde{T}_{i, j}-\widetilde{T}_{i-1, j}}{\Delta x} ; \quad \text { if } \quad q_{x}>0 \\
& \left(\frac{\partial \widetilde{T}_{d}}{\partial \eta}\right)_{i, j}=\frac{\widetilde{T}_{i+1, j}-\widetilde{T}_{i, j}}{\Delta x} ; \quad \text { if } \quad q_{x}<0
\end{aligned}
$$

and similarly for $\partial \widetilde{T}_{d} / \partial \xi$. Substitution of (179) into (177) gives the temperature derivative within an element, which is represented by discrete temperatures not only on the element, but also on neighboring elements. Green's function in (176) is exactly calculated within the elements. An adaptive Gaussian quadrature is used for integration. On each element, 4-point and 9-point rules are applied and results are compared for convergence. If the accuracy is not satisfactory, the element is continuously subdivided into smaller ones for integration until a specified accuracy is reached. The resultant linear system contains discrete nodal temperatures as the unknowns. An LU decomposition scheme is then employed to solve the matrix.

The solution obtained above is in the Laplace transform domain. It is necessary to transform the solution back into the time domain. This is achieved by using an approximate Laplace inversion method. The widely used Stehfest method [113], [82] is adopted for the purpose.

\section{Numerical examples}

Infinite Fracture We first solve the problem of an infinite planar fracture with a single injection well located at the origin (Figure 85). The initial reservoir temperature is at a constant $T=T_{o}$. At $t=0^{+}$, water at a temperature $T_{i n j}$ is injected at a flow rate $Q$. Due to axial symmetry, the fluid flow in the infinite fracture is easily resolved as:

$$
q_{r}=\frac{Q}{2 \pi r}
$$

For the numerical solution, the infinite region is truncated using a circle that is large enough to enclose the temperature deficit front to ensure that the boundary effect is small (Figure 85). The following data are 
used in the simulation [100]

$$
\begin{gathered}
Q=1 \times 10^{-2} \mathrm{~m}^{3} / \mathrm{sec} ; \quad \rho_{w}=1000 \mathrm{~kg} / \mathrm{m}^{3} ; \quad \rho_{r}=2700 \mathrm{~kg} / \mathrm{m}^{3} ; \\
K_{r}=2.0 \mathrm{~W} / \mathrm{m}^{\circ} \mathrm{K} ; \quad c_{w}=4.2 \times 10^{3} \mathrm{~J} / \mathrm{kg}^{\circ} \mathrm{K} ; \quad c_{r}=1.0 \times 10^{3} \mathrm{~J} / \mathrm{kg}^{\circ} \mathrm{K}
\end{gathered}
$$

In Figure 86 we plot the normalized temperature deficit $T_{d}$ on the fracture $(z=0)$ along the radial direction $r$, at times $t=1 \times 10^{7}, 1 \times 10^{8}$, and $1 \times 10^{9}$ sec., using symbols connected by dashed lines.

To validate the solution, it is desirable to compare the numerical result with known analytical solution. Unfortunately, it is not to our knowledge that any analytical solution simulating three-dimensional heat flow exists. Hence only an indirect comparison can be made using a one-dimensional reservoir heat flow model, which is briefly presented below.

The temperature in the fracture varies in the radial direction only, hence (155) simplifies to:

$$
\frac{Q \rho_{w} c_{w}}{2 \pi r} \frac{\partial T_{d}(r, 0, t)}{\partial r}-\left.2 K_{r} \frac{\partial T_{d}(r, z, t)}{\partial z}\right|_{z=0^{+}}=0
$$

Assuming that the heat conduction in the reservoir is one-dimensional and perpendicular to the fracture surface, (154) becomes:

$$
K_{r} \frac{\partial^{2} T_{d}(r, z, t)}{\partial z^{2}}=\rho_{r} c_{r} \frac{\partial T_{d}(r, z, t)}{\partial t}
$$

The normalized temperature deficit solved from the above set of equations is:

$$
T_{d}(r, z, t)=\operatorname{erfc}\left[\left(\frac{\pi K_{r} r^{2}}{Q \rho_{w} c_{w}}+\frac{z}{2}\right) \sqrt{\frac{\rho_{r} c_{r}}{K_{r} t}}\right]
$$

where erfc is the complementary error function. In Figure 86 we plot the normalized temperature deficit $T_{d}$ on the fracture $(z=0)$ along the radial direction $r$, at times $t=1 \times 10^{7}, 1 \times 10^{8}$, and $1 \times 10^{9}$ sec; the analytical solution is shown in solid lines and the three-dimensional BEM results are shown using dashed lines with data markers. We observe that the two solutions are very close to each other at early times. As time increases, the difference between them becomes more noticeable, but is still small. Hence the three-dimensional heat flow effect is insignificant for this infinite fracture case.

Circular Fracture In the second example, heat extraction in a circular fracture is studied. In this case, the fluid flow is no longer simple. Nevertheless, the fluid flow in the fracture induced by multiple injection/extraction wells can still be presented in an exact solution, which is constructed using the method of images [114]. For a fracture of radius $R$ centered at the origin and containing any number of injection and extraction wells, the discharge per unit width is given by:

$$
\begin{aligned}
& q_{x}=\frac{1}{2 \pi} \sum_{i=1}^{n_{w}} Q_{i}\left[\frac{x-x_{i}}{\left(x-x_{i}\right)^{2}+\left(y-y_{i}\right)^{2}}+\frac{r_{i}^{2}\left(x r_{i}^{2}-x_{i} R^{2}\right)}{\left(x r_{i}^{2}-x_{i} R^{2}\right)^{2}+\left(y r_{i}^{2}-y_{i} R^{2}\right)^{2}}\right] \\
& q_{y}=\frac{1}{2 \pi} \sum_{i=1}^{n_{w}} Q_{i}\left[\frac{y-y_{i}}{\left(x-x_{i}\right)^{2}+\left(y-y_{i}\right)^{2}}+\frac{r_{i}^{2}\left(y r_{i}^{2}-y_{i} R^{2}\right)}{\left(x r_{i}^{2}-x_{i} R^{2}\right)^{2}+\left(y r_{i}^{2}-y_{i} R^{2}\right)^{2}}\right]
\end{aligned}
$$

where $Q_{i}$ is the fluid extraction rate of the $i^{t h}$ well, $x_{i}$ and $y_{i}$ denote the well location, $n_{w}$ is the number of wells, and:

$$
r_{i}=\sqrt{x_{i}^{2}+y_{i}^{2}}
$$




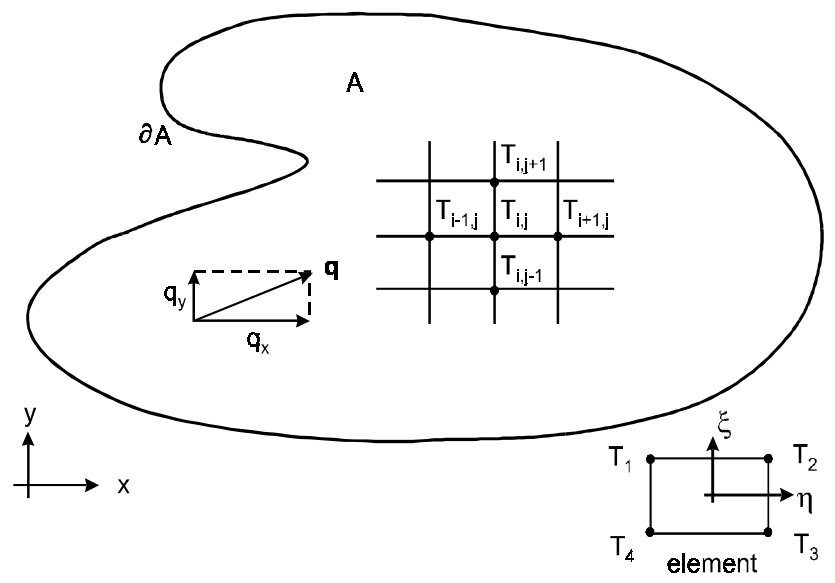

Figure 84: Computational mesh for a planar fracture.

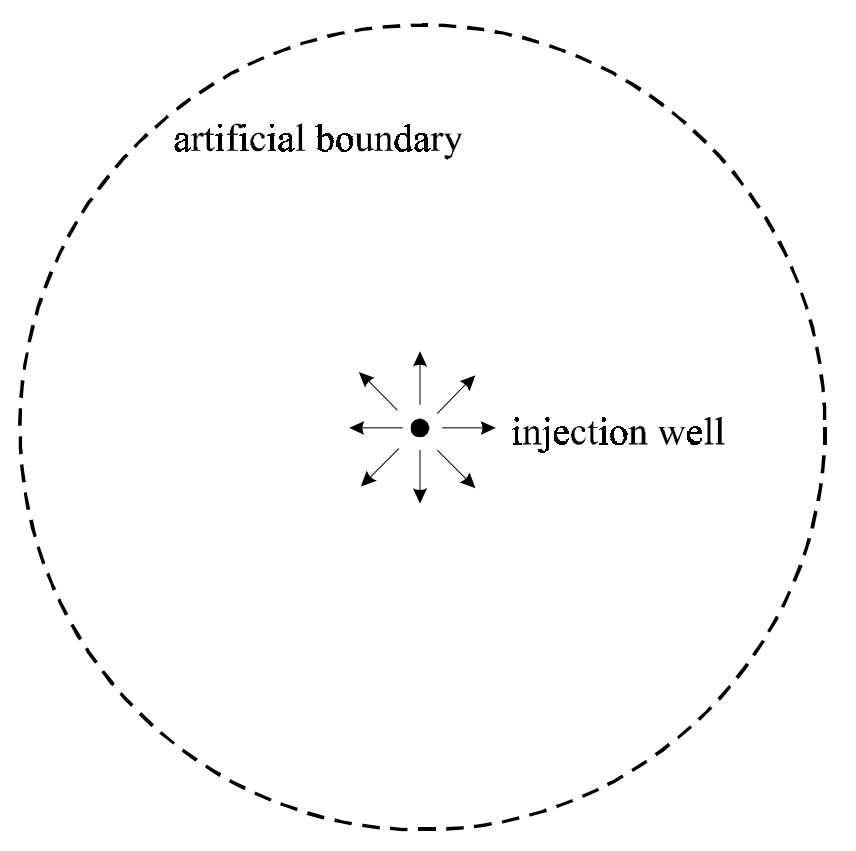

Figure 85: An infinite fracture with an injection well. 


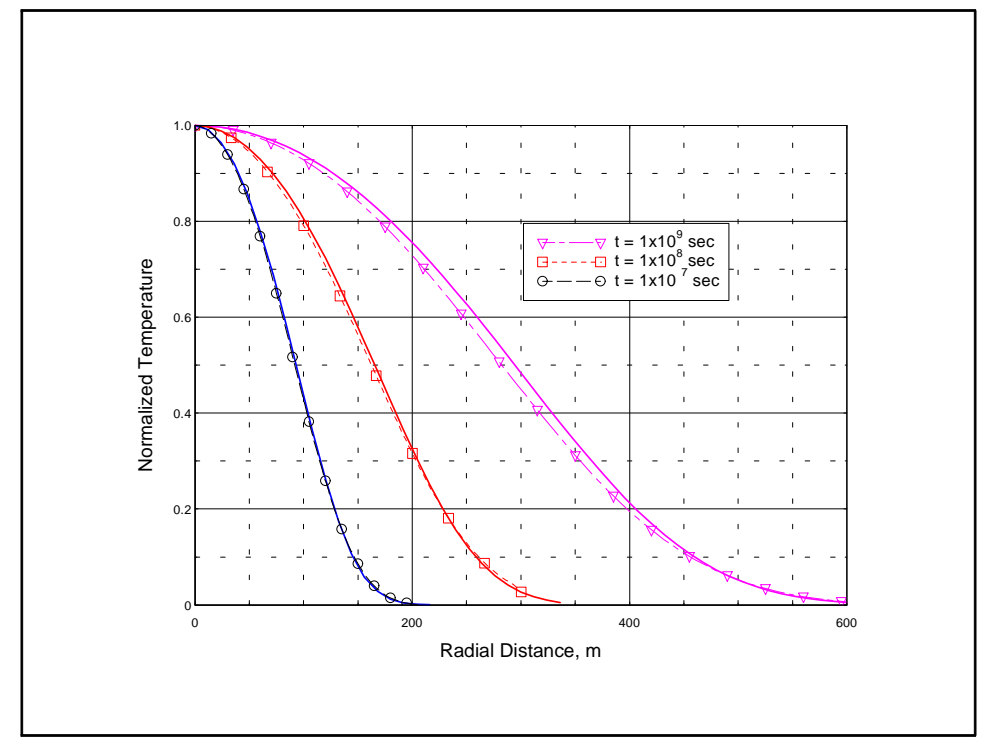

Figure 86: Normalized temperature distribution in the infinite fracture for various times.

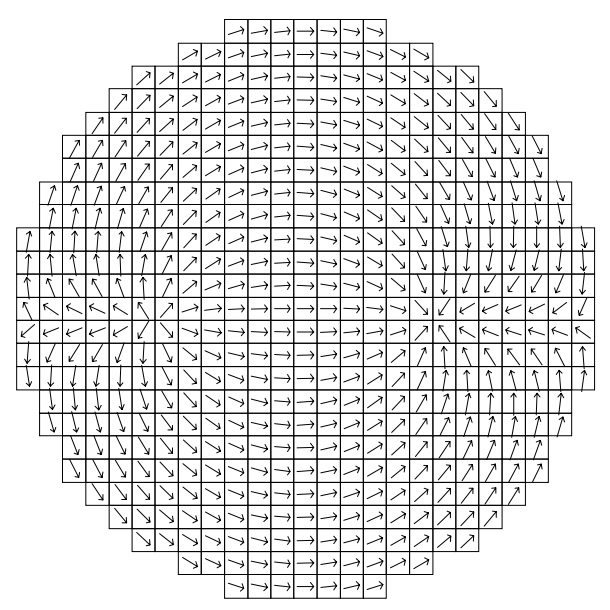

Figure 87: Computational mesh for heat extraction from a circular crack showing flow vectors. 


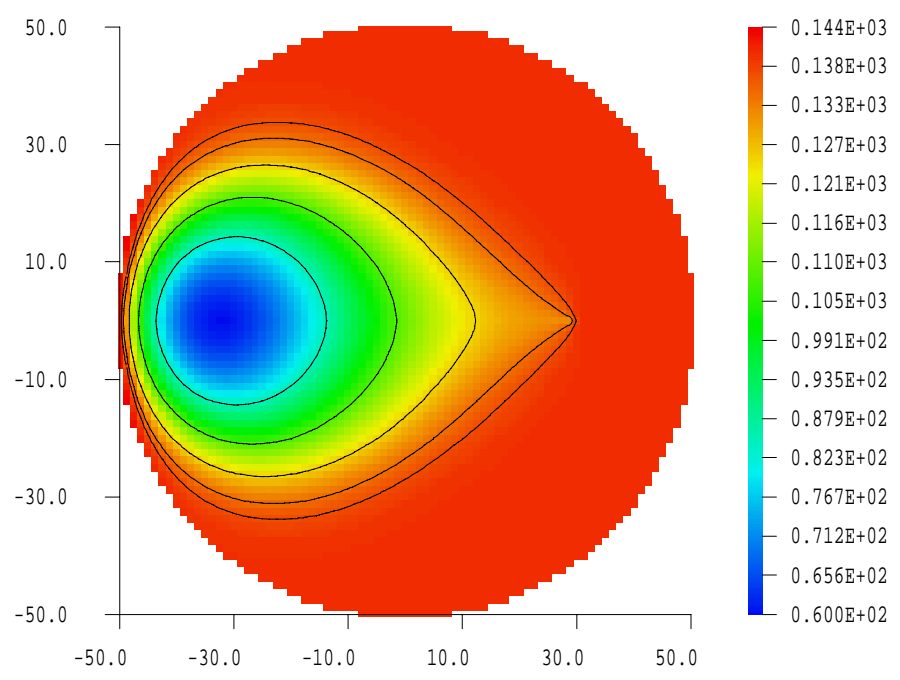

Figure 88: Temperature distribution in circular fracture at $t=3$ days, based on integral equation solution.

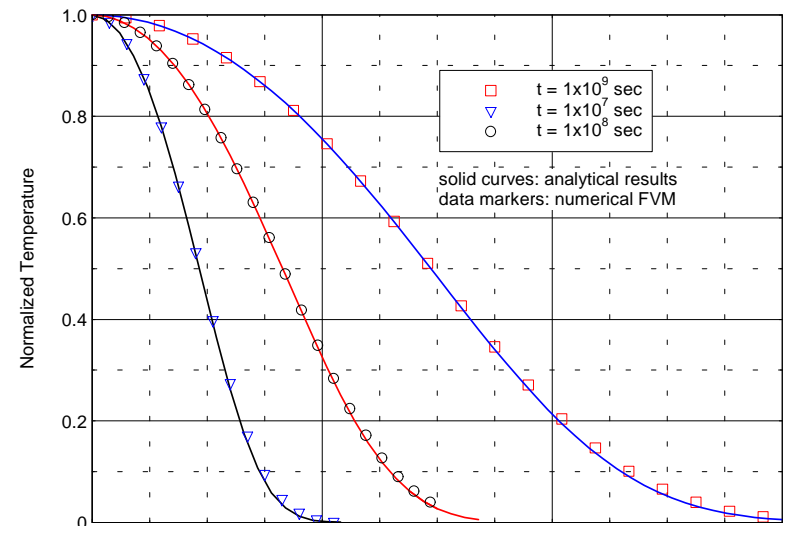

Figure 89: Comparison of the 1-D FVM and analytical results. 


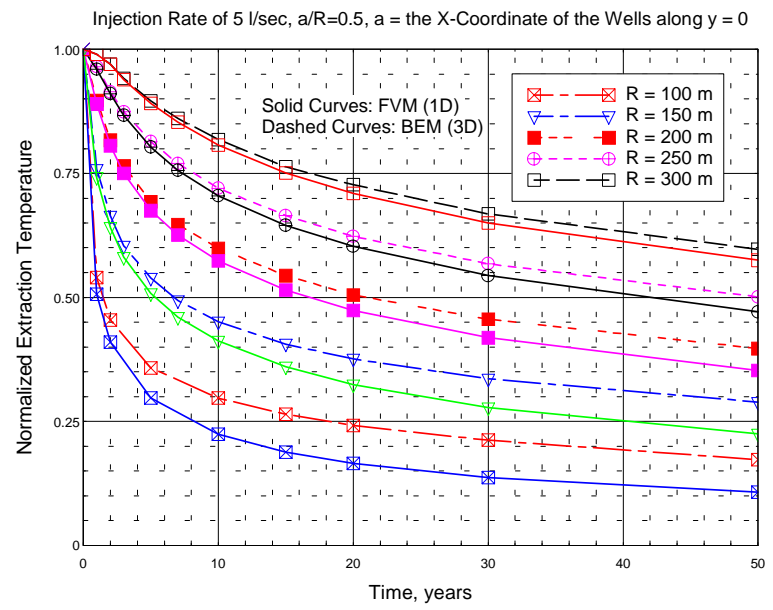

Figure 90: Extraction temperature for various fracture sizes.

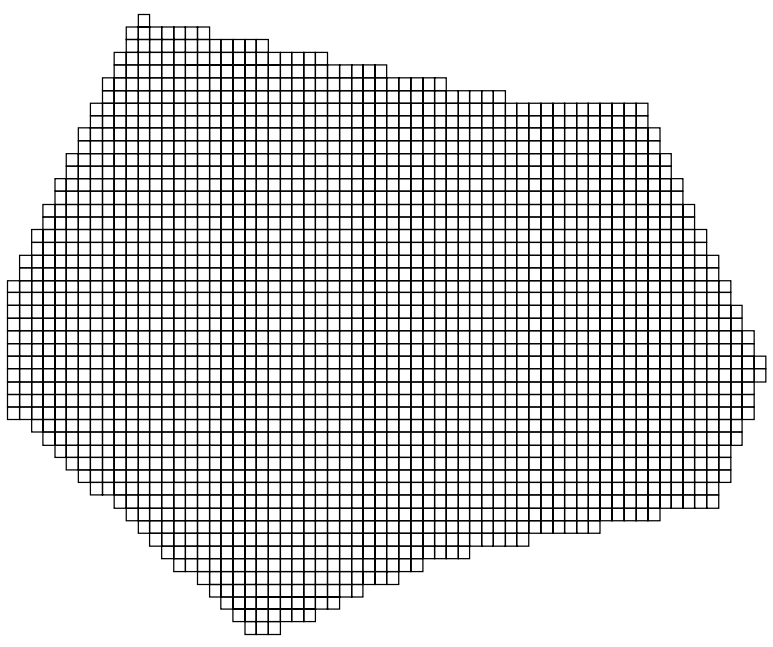

Figure 91: A fracture with an arbitrary geometry. 


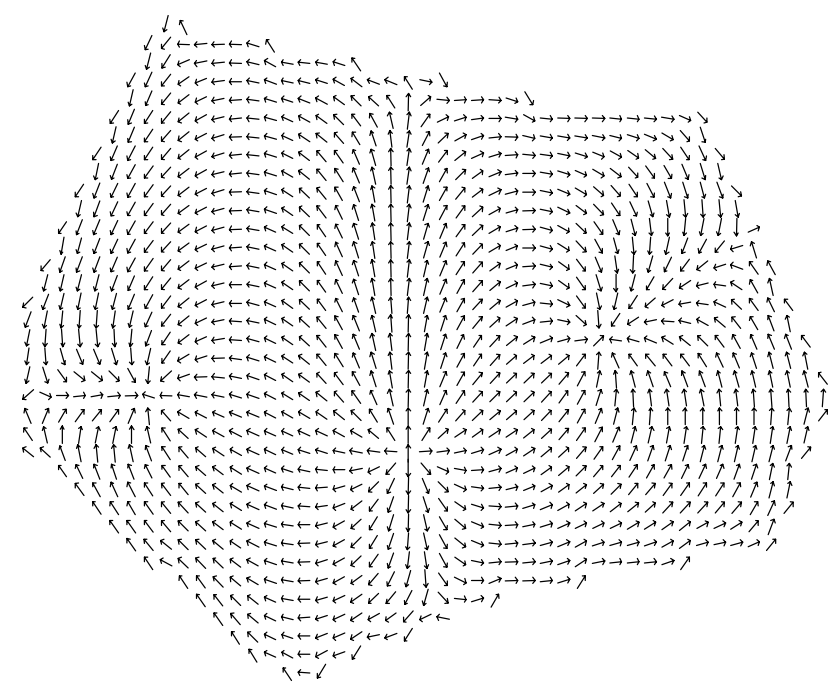

Figure 92: Fluid flow in an arbitrarily shaped fracture with one injection well and two extraction wells.

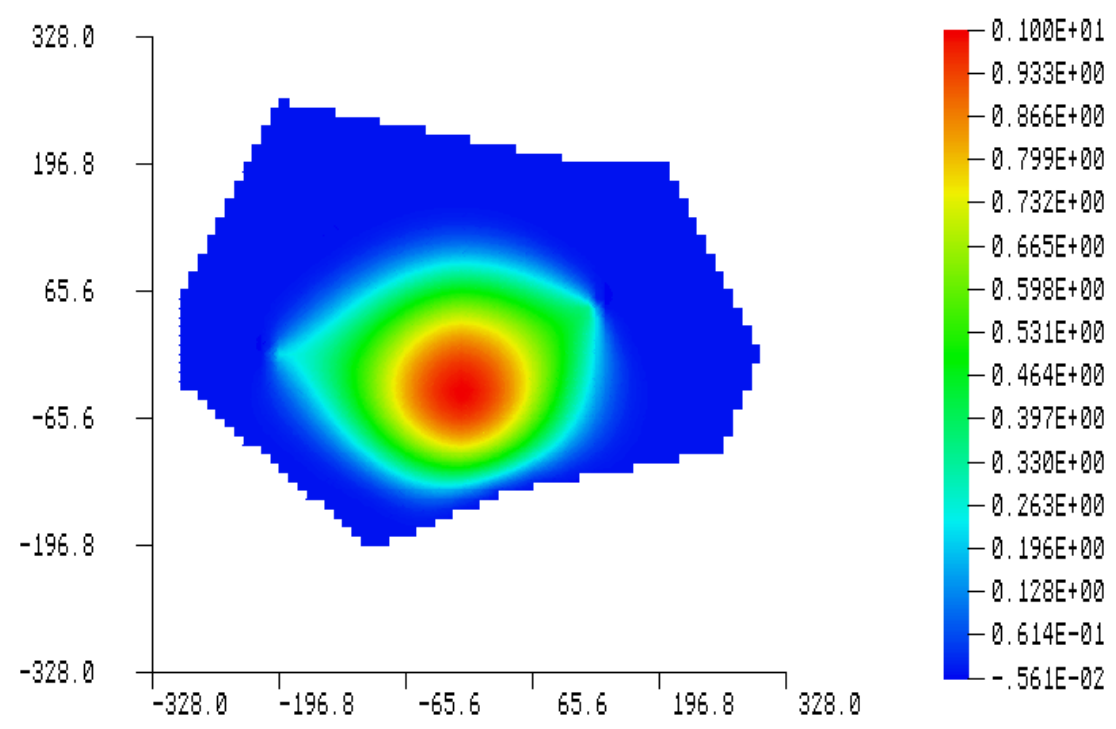

Figure 93: Normalized temperature deficit in a fracture with an injection well and two extraction wells. 
Due to the condition of mass balance of incompressible fluid, it is necessary that the extraction/injection rates sum to zero:

$$
\sum_{i=1}^{n_{w}} Q_{i}=0
$$

It is easy to check that the above solution (184) and (184) satisfies the continuity equation (43), and also the boundary condition that normal flux vanishes at the edge of the fracture, $q_{n}=0$ at $r=R$.

In this example, we solve the problem of a pair of injection and extraction wells located at $(-a, 0)$ and $(+a, 0)$ with equal but opposite discharge rate $(-Q$ and $+Q)$. The resultant flow field is illustrated in Figure 87 for the fracture radius of $50 \mathrm{~m}$ and the spacing between two wells equal to $62.5 \mathrm{~m}$.

For the solution of temperature, the fracture surface is discretized into 5024 four-noded bilinear elements similar to those shown in Figure 87. The following data set used in Rodemann [115] is adopted for the numerical solution:

$$
\begin{gathered}
Q=5 \times 10^{-3} \mathrm{~m}^{3} / \mathrm{sec} ; \quad K_{r}=2.58 \mathrm{~W} / \mathrm{m}^{\circ} \mathrm{K} ; \\
\rho_{w}=1000 \mathrm{~kg} / \mathrm{m}^{3} ; \quad \rho_{r}=2650 \mathrm{~kg} / \mathrm{m}^{3} ; \\
c_{w}=4.05 \times 10^{3} \mathrm{~J} / \mathrm{kg}^{\circ} \mathrm{K} ; \quad c_{r}=1.1 \times 10^{3} \mathrm{~J} / \mathrm{kg}^{\circ} \mathrm{K}
\end{gathered}
$$

The initial reservoir temperature is $140^{\circ} \mathrm{C}$, and the injected water temperature is $60^{\circ} \mathrm{C}$. In Figure 88 , we plot the water temperature in the fracture in isothermal lines at the time $t=3$ days. Note that the isolines correspond to $85^{\circ} \mathrm{C}, 108^{\circ} \mathrm{C}, 125^{\circ} \mathrm{C}, 134^{\circ} \mathrm{C}$, and $139^{\circ} \mathrm{C}$.

Next, we examine the effect of fracture size on the extraction temperature. In Figure 90, we present the time history of normalized extraction temperature $\left(T_{\text {extr }}-T_{i n j}\right) /\left(T_{o}-T_{i n j}\right)$ for different fracture sizes, $R=100,150,200,250$, and $300 \mathrm{~m}$. The injection and extraction wells are located at $\pm R / 2$ on the $x$-axis. The integral equation solution is presented in dash lines. We clearly observe the trend that the larger the fracture size, the higher the extraction temperature, due to longer residence time.

To compare the effect of three-dimensional versus one-dimensional reservoir heat flow model, we have separately written a Finite Volume Method (FVM) program using the one-dimensional heat flow assumption. The numerical results based on the FVM are plotted in solid lines in Figure 90. We observe that the 1-D model always under-predicts the extraction temperature. This is a consequence of the fact that the part of the reservoir beyond the fracture footprint is effectively cut off from heat supply in the one-dimensional model. The difference between the two models is more significant for smaller fracture sizes. This observation is consistent with the infinite fracture case. The result demonstrates that the use of correct reservoir geometry is important in predicting the life of HDR reservoir with a finite fracture size.

Arbitrary Shape Fracture As a last example, we consider a fracture with an arbitrary geometry. Figure 91 shows the fracture and its discretization into surface elements. The heat extraction operation involves 3 wells: one injection well with flow rate $10 \ell / \mathrm{s}$, and two extraction wells each with a flow rate of 5 $\ell / \mathrm{s}$. All other reservoir parameters are the same as the preceding example.

In this case, the fluid flow can no longer be calculated by analytical solution. The Finite Element Method is utilized to solve the potential flow in the two dimensional fracture geometry. The flow singularities were treated using a singularity extraction and superposition procedure [116]. The resultant flow field is plotted using velocity vectors in Figure 92. The normalized temperature deficit in the fracture after 6 months of operation is shown in contour lines in Figure 93. 
In summary, an integral equation formulation for heat extraction from a planar fracture in an infinite hot dry rock reservoir has been presented. Most of the existing work, analytical and numerical, treats the heat conduction in the geothermal reservoir as one-dimensional and perpendicular to the fracture. This assumption significantly restricts the heat supply to the HDR system, hence under-predicts the system's performance. One of the reason that the three-dimensional nature of heat conduction is ignored is the difficulty in treating the infinite geothermal reservoir geometry by numerical discretization.

In the present work, we demonstrate that by the use of three-dimensional Green's function for heat conduction and the integral equation formulation, the need for discretizing the three-dimensional reservoir is completely eliminated. The resultant discretization involves the fracture surface only.

To demonstrate the solution algorithm, three examples, an infinite fracture with a single injection well, a circular fracture with a pair of injection and extraction wells, and an arbitrarily-shaped fracture with one injection and two extraction wells, were solved. Unfortunately, due to the lack of analytical solution that models the three-dimensional heat conduction, comparisons were made only with an analytical solution and a FVM solution, both with one-dimensional heat conduction assumption. Partial validation is achieved by observing the consistency in asymptotic behavior as the fracture size becomes large. The proposed methodology is then used to solve HDR heat extraction system involving an arbitrarily-shaped fracture with multiple injection and extraction wells. Numerical investigation shows that the three-dimensional heat conduction can significantly alter the prediction of heat extract temperature and the reservoir life, particularly for smaller fractures.

Although the present integral equation solution has been applied to a single fracture, the same concept can be applied to a reservoirs with a number of fractures. Other improvement that can be implemented in the model includes the modeling of reservoir elasticity and thermoelasticity. 


\section{Conclusions \& Future Research}

\subsection{Heat Extraction \& Thermoelastic Effects}

In this portion of the work several mechanisms of heat transport by fluid flow in a fracture were examined. An analytical solution that incorporates all the mechanisms was presented. By a numerical example, it was demonstrated that the heat storage and the heat dispersion effects are not important due to dominant advection in the fracture fluid flow.

Then, the heat extraction problem was formulated in 2D and 3D for line cracks and planar fractures, respectively. Most existing works, analytical and numerical, treat the heat conduction in the geothermal reservoir as one-dimensional and perpendicular to the fracture. This assumption significantly restricts the heat supply to the HDR system, hence under-predicts the system's performance. For the two-dimensional model, an integral equation formulation was derived such that the need for modeling the reservoir geometry was eliminated in the discrete solution system. Numerical investigation showed that the two-dimensional heat conduction can significantly alter the prediction of heat extraction temperature and the reservoir life.

The same approach was then applied in a three-dimensional geometry to eliminate the discretization of geothermal reservoir. The integral equation formulation for heat extraction from a planar fracture in an infinite hot dry rock reservoir was derived. To our knowledge this is the only numerical solution for 3D heat extraction that agrees with analytical results. The proposed methodology was then used to solve an HDR heat extraction system involving an arbitrarily-shaped fracture with multiple injection and extraction wells. Numerical investigation has shown that the three-dimensional heat conduction significantly alters the prediction of heat extract temperature and the reservoir life, particularly for smaller fractures. Although the present integral equation solution has been applied to a single fracture, the same concept can be applied to a reservoirs with a number of fractures. Other improvement that can be implemented in the 3D model includes the modeling of reactive fluid flow and joint slip.

The thermoelastic effects associated with fluid injection into a fracture were also studied in this work. The two-dimensional injection/operation scheme has been implemented in the mechanical fracture propagation program. A 2D constant element real displacement discontinuity model was used to treat the problem in $2 \mathrm{D}$. The thermal stresses were calculated from the source strengths obtained from the $2 \mathrm{D}$ boundary element solution of heat extraction. It was noted that significant stresses are generated on the facture face that increase with time. This suggests that when compared to pore pressure, the thermal stresses play a more significant role in joint and fault slip and associated seismicity.

The thermal stress numerical algorithms combined with the fracture propagation models allow one to calculate slip on the fractures due to thermally-induced variations of in-situ stress. One can also study the impact of long term injection on stress distribution in the reservoir. Due to time limitations, however, final

verification and examples were not presented. The modifications of the interface to allow consideration of such problems along with some numerical examples will be accomplished in the next phase of work.

The aforementioned thermoelastic study was based on the assumption of rock homogeneity. The presence of inhomogeneities has recently been addressed [137] by developing a steady-state CV-BEM thermoelastic model; its extension to include transient effects is forthcoming. 


\subsection{Wellbore Failure and Fracture Initiation; Porothermoelastic Effects}

When drilling in a low permeability geothermal reservoir, the transient temperature distribution in the formation may be approximated by conductive heat transfer. The formation is gradually cooled when the borehole temperature is suddenly reduced. A significant pressure drop is generated near the borehole at early times. With time, the pore pressure peak is reduced and moves away from the well where it gradually recovers the original state. A significant radial tensile stress peak is produced inside the formation, and the tensile stress zone moves inside the formation while the magnitude of the "peak" increases. Tensile tangential stresses are induced around the wellbore due to the tendency of the rock to shrink when cooled. Away from the borehole wall, the magnitude of the induced tensile stress decreases and changes its sign at some point inside the formation, i.e., it becomes compressive. This is because the shrinkage of the material near the borehole due to cooling tends to pull on the outer rock thus inducing a compressive stress zone that gradually moves away from the borehole.

By considering the total pore pressure, tangential, and radial stress (the sum of the induced and in-situ stress) distribution, it has been found that the maximum tensile (tangential) stress always occurs at the wall, but a zone of tension also develops and extends into the formation with increasing cooling time.

In summary, high tensile stresses are developed around the well as a result of cooling. The tensile zone expands into the reservoir with time resulting in fracture initiation and possible propagation. Thus, one can take advantage of thermal stresses to stimulate geothermal reservoirs.

\subsection{Fracture Propagation}

A CV-BEM was developed and used to model various fracture propagation problems. In this approach, the displacement discontinuities are defined from the numerical solution of a complex hypersingular integral equation written for the fracture of a given configuration and loading. The main advantages of the complex variable approach are: the ability to consider fractures in piecewise homogeneous media, i.e., to take into account inclusions and openings; the use of a circular arc (straight segment as a degenerated case) to model the crack path increment at each propagation stage; the use of high order approximating polynomials with square-root asymptotics for the unknown functions (DD); and the use of a circular arc (straight segment) for segments of the crack path that allows one to avoid the numerical integration under the solution of the hypersingular integral equation. This leads to high accuracy in the calculation of the stress intensity factors.

The model was applied to study several aspects of crack propagation from a wellbore. The variation of propagation pressure along various paths was considered first. It was found that although the path of the fracture is independent of the mean stress, the propagation pressure does dependent on it. The corresponding pressure for every path attains a minimum at a point corresponding to the inflection point of the propagation path. When the inflection point does not exist, the pressure curve displays no minimum. It was observed that the orientation of the initial fracture has a strong effect on the fracture path. The fracture tends to return to what would be the path of the "favorably oriented fracture". The propagation pressure drops to a rather low value irrespective of the orientation of the preexisting fracture. In general, higher values of the stress ratio lead to lower minimum values of the propagation pressure.

Computations have shown that the fracture path is practically independent of the length of fracture increment. For a fixed set of stresses, fracture toughness, and wellbore radius, the fracture path is only weakly dependent on the initial fracture length. However, the initiation pressure is strongly dependent on the initial fracture length for all initial orientations of the fracture. 
For fracture propagation from a wellbore, there is a tremendous difference between the results for the schemes of slow and fast pressurization. When calculations are carried out without fluid pressure penetrating the crack, fractures generally follow the path along the initial radial direction with little curvature. Pressures required to propagate the fracture are high and increase rapidly with the fracture length. This type of behavior has been observed in laboratory experiments.

The Complex Variable Hypersingular Boundary Element Method has also been used for studying hydraulic fracturing near natural fractures, faults, and inhomogeneities. We have found that in all the cases studied, both with and without a fault, the trajectory of the crack tends to be aligned in the direction of the maximum compressive principal stress acting in the vicinity of a wellbore. A fault influences the crack propagation by altering the stresses in the vicinity of the wellbore from those at infinity, a propagating crack noticeably influences tractions and displacement discontinuities on a fault when approaching at distances less than the wellbore radius. We have demonstrated that natural fractures, faults, and other inhomogeneities generate unstable fracture configurations which can transform significantly under small disturbances of the initial crack inclination, loading, and geometry. The fault inclination and frictional characteristics as well the conditions on the fracture surfaces proved to be the most significant factors that influence the trajectories.

\subsection{Graphics User Interface}

In order to maximize the efficacy to the industry, our design codes and analytical solutions have been developed with a graphics user interface (GUI) to enhance the fracture propagation modeling capabilities. In short, GUI is a graphical representation of the software that gives it a friendly look and helps the user to experience the "feel" and "power" of the software. The user interface has graphics routines, and dialog boxes for conveniently selecting a desired program and entering data. Graphics capabilities have been implemented to assist the user in problem definition. Post-processing routines allow program output to be viewed directly through graphic presentations or saved in data files for later use. The fracture propagation model can be accessed through client-server architecture. The server, as the name suggests, provides services to clients. In a typical case, client-server architecture consists of one server and one or more clients. The services can range from performing a simulation requested by the client to "asking" other client(s) connected to that system to conduct simulations and to provide the results to the client. This is called a distributed computing system since there is a group of computers connected together and working "for" each other, with the server managing all the ongoing network activities. If an application needs to plot graphs using a set of values, then the application can be broken into two parts: a part that calculates the necessary values and the other part which plots them. The function (or program) that performs the calculations can be placed on the server allowing the clients to implement the plotting function. Since the main function is placed on the server, the client needs only to log into the remote site and run the graphics application Thus, the actual implementation of the application is transparent to the user. In this way, it is not necessary to distribute

the program. However, a copy of the server program can be provided to the client through the download option.

\subsection{Practical Guidelines for Enhanced Geothermal Systems}

The models developed in this study allow one to perform quantitative analyses when considering various rock mechanics issues in planning and development of enhanced geothermal systems. The work completed to date demonstrates a number of practical guidelines for geothermal reservoir development: 


\subsubsection{Drilling}

The drilling of a wellbore is a continual motion (ideally) that leads to gradual deepening of the wellbore. Initially, the relatively cool mud (and bit) comes in contact with increasingly warmer rocks (with depth). This leads to cooling of the formation near the wellbore. Cooling reduces the total stresses in the rock near the well (reducing the stress difference, i.e., Mohr's circle radius) and induces a pore pressure drop inside the formation that increases the effective stresses near the wellbore. As time increases, the mud temperature will equilibrate with its surroundings so that the formations higher in the section being drilled are subjected to the increased temperature of the mud. Heating generates increased pore pressure and total stresses near the wellbore, the former reduces the effective stresses. These processes may be occurring simultaneously in the lower and upper segments of the wellbore, respectively. Cooling can cause wellbore stability with respect to shear failure and instability with respect to tensile failure. The latter mechanism is useful in fracture initiation in stimulation; cold fluid injection can also significantly enhance fracture permeability.

\subsubsection{Fracture initiation/propagation}

Modeling the propagation of fractures with various lengths and orientations clearly demonstrates that wellbore orientation can have an impact on future simulation practices. Trajectories of inclined wells need to be optimized in relation to future stimulation needs and the current state of stress to facilitate fracturing. Also, perforations need to be oriented favorably with respect to the in-situ stress direction. Perforations oriented close to the direction of maximum in-situ stress minimize near wellbore restrictions and can facilitate hydraulic stimulation.

\subsubsection{Fracturing of naturally-fractured reservoirs}

Hydraulic fracturing can be successfully applied in naturally fractured reservoirs. The path of the fracture is not a single plane but involves propagation along and through existing natural fractures. However, a hydraulic fracturing treatment may not be successful if the existing fracture orientations are parallel to the current maximum horizontal stress. In this situation, the hydraulically induced fractures will most likely propagate parallel to the natural fractures with little connectivity with fractured zones. It may be better to orient the wellbore to intersect the natural fractures in the rock mass. In general, whether an artificial fracture can effectively propagate through a fractures rock mass depends on the joint properties, the in-situ stress state, and injection rate. Competent natural fractures with high normal stresses promote fractures intersection and crossing. The analysis has shown that when the fluid does not penetrate the fracture, it tends not to be reoriented in the direction of maximum stress and may intersect existing natural cracks. Such pressurization scheme can be achieved in sleeve fracturing and dynamic fracturing by explosives. In an extensively fractured reservoirs, hydraulic stimulation can be used to connect the wellbore to the permeable fractured zones.

\subsubsection{Influence of cold fluid injection}

The results have also shown that cold fluid injection in the reservoir can alter the stresses significantly and reduce the effective normal stress on joints. As a result, fracture slip is highly likely and can lead to permeability enhancement. Extensive slip, however, may cause a permeability reduction by crushing the aspirities. Furthermore, cooling can impact interpretation of hydraulic fracturing for determination of in-situ 
stress. The breakdown pressure is reduced by the cooling induced tensile tangential stresses. Furthermore, cooling a larger region around the wellbore will reduce the stresses so that using the instantaneous shut-in pressure will underestimate the value of the minimum in-situ stress. The latter should be considered when measuring stresses in a hole that has been drilled for other purposes.

\subsection{Future Work}

The next phase of the work will include implementation of the current capability in a reservoir geomechanics model for calculating stress and fracture data for use in reservoir characterization and development plans and for stimulation modeling. The work will allow a priori determination of suitable target zones, drilling risks and alternatives, and assessment of stimulation by hydraulic fracturing. The proposed geomechanics model will be developed through rock mechanics research and development activities for EGS namely:

- Enhancement of the current 2D fracture propagation model to allow analysis of thermal stresses while considering rock inhomogeneities, generation of fracture networks, and fracture interactions, and fracture slip.

- Formulation and solution of the fracture arrest or "jog" at the interface should follow the analysis of the fracture trajectory once it is determined whether it is attracted or rejected by the interface. Future improvements should address a more general concept of interaction, i.e., consideration of the successive process of loss of cohesion on the fault surface as the fracture propagates towards it. Also, special asymptotics need to be implemented near the tip depending on the interface characteristics near the tip. At last, the question of fracture process zone and its possible effects of fault local opening should be studied.

- Three-dimensional calculation of cooling induced stresses and 3D analysis of natural fracture response

Furthermore, the reservoir's state of stress is of fundamental importance to many aspects of geothermal energy development. Fracture distribution is largely controlled by stress, which in turn depends on rock rheology, natural discontinuities such as faults, poroelastic, and thermal stresses. The quantification of the stress state is also important in other aspects of reservoir development, namely optimum drilling trajectory, borehole instability and fluid loss which adversely impact economic drilling of geothermal wells; and hydraulic fracturing. Thus, the following activities are also recommended:

- Development of a 2D poro-viscoelastic finite element model with damage mechanics for predicting zones of fluid accumulation and deformation-induced fluid flow and migration. The geomechanics model will allow:

- Prediction of the in-situ stress distribution, including mean, maximum, and minimum stresses (and) strains as well as the pore fluid pressure and temperature

- Prediction of fracture zones as well as fracture type and orientation associated with reservoir deformation

- Development of a state-of-the-art porothermoelastic wellbore stability model to assist in planning drilling operations, and hydraulic fracturing for future reservoir development

The models will be applied in a quantitative geomechanics study of geothermal fields of interest such as Coso. This will allow calibration of the model for application to future sites. 


\section{References}

[1] Hubbert M.K. \& Willis D.G., 1957. Mechanics of hydraulic fracturing. Trans. Am. Inst. Min. Engrs., 210, 153-168.

[2] Brace, W.F., 1960. An extension of the Griffith's theory of fracture to rocks. J. Geophys. Res., 65(10), 3477-3480.

[3] Brace, W.F., 1964. Brittle fracture of rocks, In W.R. Judd (ed.) State of Stress in the Earth's Crust. Elsevier, New York, 111-174.

[4] Callanan, M.J., 1981. Hydraulic fracture initiation by shear failure in formations at great depths. Proc. Workshop on hydraulic fracturing stress measurements, Monterey, CA, 181-189.

[5] Guo, F., Morgenstern, N.R. \& Scott, J.D., 1993. Interpretation of hydraulic fracturing breakdown pressure. Int. J. Rock Mech. Min. Sci. \& Geomech. Abstr., 30(6), 617-626.

[6] Clausing, D.P., 1959. Comparison of Griffith's theory with Mohr's failure criteria. Quart. Colo. School of Mines, 54, 286-296.

[7] Daneshy, A.A., 1989. Proppant transport, In Recent Advances in Hydraulic Fracturing, SPE monograph, 12, 210-222.

[8] England, A.H. \& Green, A.E., 1963. Some two-dimensional punch and crack problems in classical elasticity. Proc. Camb. Phil. Soc., 59, 489-500.

[9] Erdogan, F. \& Sih, G.C., 1963. On the crack extension in plates under plane loading and transverse shear. Trans. ASME, J. Basic Engrg., 519-527.

[10] Erdogan, F. 1974., Principles of fracture mechanics. In Thoft-Christensen (ed.) Continuum Mechanics Aspects of Geodynamics and Rock Mechanics, D. Reidel publishing, Dordrecht-Holland, 29-44.

[11] Goodier, J.N. 1972. Mathematical theory of equilibrium cracks. In H. Liebowitz (ed.) Fracture: An Advanced Treatise, 2, 1-66.

[12] Goodman, R.E., 1980. Introduction to rock mechanics. John Wiley \& Sons, New York.

[13] Daneshy, A.A., 1973. Experimental investigation of hydraulic fracturing through perforations. J. Pet. Tech., Oct., 1201-1206.

[14] Griffith, A.A., 1921. The theory of rupture and flow in solids. Phil. Trans. Roy. Soc. London, 163-197.

[15] Griffith, A.A., 1924. Theory of rupture. Proc. of the 1st Int. Cong. Appl. Mech., Delft, 55-63.

[16] Rinehart, J.S., 1966. Fracture of rocks. Int. J. Fract. Mech., 2(3), 534-551.

[17] Murrell, S.A.F., 1958. The strength of Coal under triaxial compression. In W.H. Walton (ed.) Mechanical Properties of Nonmetallic Solids, Interscience, New York, 123-146.

[18] Harr, M.E., 1962. Ground water and seepage. McGraw-Hill, New York. 
[19] Howard, G.C. \& Fast, C.R., 1970. Hydraulic fracturing. SPE monograph, Vol. 2, Henry L. Doherty series.

[20] Hussain, M.A., Pu, S.,L. \& Underwood, J., 1974. Strain energy release rate for a crack under combined Mode I and Mode II. In Fracture Analysis, Proc. of the '73 Nat. Symp. on fracture mechanics, part II, ASTM STP 560, ASTM, 2-28.

[21] Inglis, C.E., 1913. Stresses in a plate due to the presence of cracks and sharp corners. Trans. Inst. Naval Architects, 55, 219-230.

[22] Irwin, G.R., 1957. Analyses of stresses and strains near the end of a crack transversing a plate. J. Appl. Mech., 24, 361-364.

[23] Roegiers, J.-C. \& Zhao, X.L., 1991. Rock fracture tests in simulated downhole conditions. Proc. of the 32nd U.S. Symposium on Rock Mechanics, Rock mechanics as a Multidisciplinary Science, A.A. Balkema, 221-230.

[24] Boone, T.J., Ingraffea, A.R. \& Roegiers, J.-C., 1991. Simulation of hydraulic fracture propagation in poroelastic rock with application to stress measurement technique. Int. J. Numer. Anal. Meth. Geomech., 28, 1-14.

[25] Jaeger, J.C. \& Cook, N.G.W., 1979. Fundamentals of rock mechanics. 3rd ed., Chapman and Hall, New York.

[26] Kanninen, M.F. \& Popelar, C.H., 1985. Advanced fracture mechanics. Oxford University Press, New York.

[27] McClintock, F.A. \& Walsh, J.B., 1962. Friction on Griffith cracks under pressure. Proc. of the 4th U.S. National Cong. of Appl. Mech., 1015-1577.

[28] Obert, L., 1972. Brittle fracture of rock. In H. Liebowitz (ed.) Fracture: An Advanced Treatise, VII, 93-155.

[29] Ong, S.H., 1994. Borehole Stability. Ph.D. dissertation, Dept. of Petroleum and Geological Engineering, The University of Oklahoma.

[30] Paulding, B.W., 1967. Orientation of hydraulically induced fractures. Proc. 9th Symp. on Rock Mech., Golden, Colorado, 461-489.

[31] Palaniswami, K. \& Knauss, W.G., 1978. On the problem of crack extension in brittle solids under general loading. In Nemat-nasser (ed.) Mechanics Today, Vol. 4, Pergamon Press, New York, 87-148.

[32] Yatomi, C., Fujii, K. \& Nakagawa, K., 1989. Combined stress hypothesis for mixed mode fracture toughness criterion. Engrg. Fracture Mech., 32(6), 881-888.

[33] Rummel, F., 1987. Fracture mechanics approach to hydraulic fracturing stress measurements. In B.K. Atkinson (ed.) Fracture Mechanics of Rocks, 217-240.

[34] Shah, R.C., 1974. Fracture under combined modes in 4340 Steel. In: Fracture Analysis. Proc. of the 1973 national symposium on fracture mechanics, part II, ASTM STP 560, ASTM, 29-52. 
[35] Schmidt, R.A., 1980. A microcrack model and its significance to hydraulic fracturing and fracture toughness testing. Proc. 21st Rock Mech. Symp., 581-590.

[36] Sih G.C. \& Erdogan, F., 1963. Stress intensity factor: concept and application. Linear Fracture Mechanics, Sih G. C., Wei, R.P., \& Erdogan F. (eds.), Envo Publishing Company, 85-106.

[37] Sih, G.C. \& Macdonald, B., 1974. Fracture mechanics applied to engineering problems-strain energy density fracture criterion. Engineering Fracture Mechanics, 6, 361-386.

[38] Sih, G.C., 1973. Some basic problems in fracture mechanics and new concepts. Engineering Fracture Mechanics, 5, 365-377.

[39] Sih, G.C., 1974. Strain-energy-density factor applied to mixed mode crack problems. Int. J. fracture, 10(3), 305-320.

[40] Sneddon, I.N., 1946. The distribution of stress in the neighborhood of a crack in an elastic solid. Proc. Royal Soc. London, Ser. A., 187, 229-260.

[41] Solberg, P., Lockner, D. \& Byerlee, J., 1977. Shear and tension hydraulic fractures in low permeability rocks. Pure Appl. Geophys., 115, 191-198.

[42] Starfield, A.M. \& Cundall, P.A., 1988. Towards a methodology for rock mechanics modeling. Int. J. Rock Mech. Min. Sci. \& Geomech. Abstr., 25(3), 99-106.

[43] Thiercelin, M., Jeffrey, R.G. \& Ben Naceur, K., 1989. Influence of fracture toughness on the geometry of hydraulic fractures. SPE Production Engrg., Nov., 435-442.

[44] Tirosh, J., 1977. Incipient fracture angle, fracture loci and critical stress for mixed mode loading. Engrg. Fract. Mech., 9, 607-616.

[45] Valko, P. \& Economides, M.J., 1993. A continuum-damage mechanics model of hydraulic fracturing. J. Pet. Tech., March, 198-205.

[46] Westergaard, H.M., 1939. Bearing pressures and cracks. J. Appl. Mech., 6(2), 49-53.

[47] Whittaker, A., (ed.), 1985. Theory and application of drilling fluid hydraulics. IHRDC Publishers, Boston, MA.

[48] Wiles, T.D., 1985. Modeling of Hydraulic fracture propagation in a discontinuous rock mass using the displacement discontinuity method. Ph.D. dissertation, University of Toronto, Toronto.

[49] Witherspoon, P.A., Wang, J.S.Y., Iwai, K. \& Gale, J.E., 1980. Validity of cubic law for fluid flow in a deformable rock fracture. Water Resources Res., 6(6), 1016-1024.

[50] Wolff, R.G., Bredehoeft, J.D., Keys, W.S. \& Shuter, E., 1975. Stress determination by hydraulic fracturing in subsurface waste injection. J. of American Water Works Association, 67, 519-523.

[51] White, Jr., F.M., Barfield, B.F. \& Goglia, M.J., 1958. Laminar flow in a uniformly porous channel. $J$. Appl. Mech., Dec., 613-617. 
[52] Barenblatt, G.I., 1962. The mathematical theory of equilibrium cracks. In: Brittle Fracture, Advances in Appl. Mech., 7, 55-129.

[53] Charlez Ph. A., 1997. Rock Mechanics (2): Petroleum Applications, Èditions Technip, Paris.

[54] Haimson B. C. \& Fairhurst C., 1967. Initiation and extension of hydraulic fractures in rocks. Soc. Pet. Eng. J., 310-318.

[55] Hardy M.P., 1973. Fracture Mechanics Applied to Rocks. Ph.D. Thesis, University of Minnesota.

[56] Zoback M.D., Rummel F., Jung R. \& Raleigh C.B., 1977. Laboratory hydraulic fracturing experiments in intact and pre-fractured rock. Int. J. Rock Mech. Min. Sci. Geomech. Abstr, 14, 49-58.

[57] Abou-Sayed A.S., Brechtel C.E. \& Clifton R. J., 1978. In situ stress determination by hydrofracturing: a fracture mechanics approach. J. Geophys. Res., 83(B6), 2851-2862.

[58] Rummel F. \& Hansen J., 1989. Interpretation of hydrofrac pressure recordings using a simple fracture mechanics simulation model. Int. J. Rock Mech. Min. Sci. Geomech. Abstr, 26, 483-488.

[59] Ito T. \& Hayashi K., 1991. Physical background to the breakdown pressure in hydraulic fracturing tectonic stress measurements. Int. J. Rock Mech. Min. Sci. Geomech. Abstr, 28, 285-293.

[60] Stephens, G. \& Voight, B., 1982. Hydraulic fracturing theory for conditions of thermal stress. Int. J. Rock Mech. Min. Sci. \&3 Geomech. Abst., 19, 279-284.

[61] Wolfe, C.A., 2002. Borehole Stability in Shale, M.Sc. Thesis, Dept. of Geology \& Geological Engineering, University of North Dakota.

[62] Detournay E. \& Cheng A.H., 1992. Influence of pressurization rate on the magnitude of the breakdown pressure. In: Proc. 33rd US Rock Mechanics Symposium, 325-333, Balkema, Rotterdam.

[63] Thiercelin M., 1992. The influence of microstructure on hydraulic fracturing breakdown pressure. In: Structure et Comportment Mècanique des Gèomateriaux; Colloque Rene Houpert, 419-428, Nancy.

[64] Atkinson C. \& Thiercelin M., 1993. The interaction between the wellbore and pressure-induced fractures. International Journal of Fracture, 59, 23-40.

[65] Carbonell R., 1994. Fracture Mechanics Analysis of the Breakdown Pressure. MSc thesis, University of Minnesota.

[66] Daneshy, A. A., 2003. Off-balance growth: a new concept in hydraulic fracturing. JPT, April, 78-85.

[67] Detournay E. \& Carbonell R., 1994. Fracture mechanics analysis of the breakdown process in minifrac or leak-off tests. Proceedings EUROCK 94 Symposium, Delft, 399-407.

[68] Mogilevskaya S.G., 1996. The universal algorithm based on complex hypersingular integral equation to solve plane elasticity problems. Computational Mechanics 18, 127-138.

[69] Mogilevskaya S.G., 1997. Numerical modeling of 2-D smooth crack growth. Int. J. Fracture, 87, 389405. 
[70] Mogilevskaya, S.G., 1998. Complex singular and hypersingular equations for the system of openings, cracks and inclusions in a half-plane. In: M. Bonnet \& Aubry, D. (ed.), Proceedings of the International Symposium on Boundary Element Methods, Ecole Polytechnique, Paris, 153-154.

[71] Linkov A. M., Mogilevskaya S. G. \& Napier J. A. L., 1997. Multiple interacting curvilinear crack problems: a method of solution and numerical results. In: Kim K., editor. Proceedings 36th US Rock Mechanics Symposium (NYRock'97), Elsevier, Oxford.

[72] Linkov, A.M. \& Mogilevskaya, S.G., 1998. Complex hypersingular BEM in plane elasticity problems. In: V. Sladek and J. Sladek, editors. Singular Integrals in Boundary Element Method, Computational Mechanics Publication, 299-364.

[73] Kitagawa, H. \& Yuuki, R., 1977. Analysis of branched cracks under biaxial stresses. In: Fracture 1977, 3, University of Waterloo Press, 201 p.

[74] Zang, W. L., 1990. On modeling of piece-wise smooth cracks in two-dimensional finite bodies. Int. J. of Fracture, 46, 41-55.

[75] Murakami, Y. (ed.), 1987. Stress Intensity Factors Handbook. Oxford: Pergamon Press.

[76] Mogilevskaya, S.G., Rothenburg, L., \& Dusseault, M.B., 2000. Interaction between a circular opening and fracture originating from its boundary in a piecewise homogeneous plane. Int. J. Numer. Anal. Meth. Geomech., 24, 947-970.

[77] Cataldi, R. \& Sommaruga, C., 1986. Background, present state and future prospects of geothermal development. Geothermics, 15(3).

[78] Taylor, G.I., 1953. Dispersion of soluble matter in solvent flowing slowly through a Tube. Proc. Roy. Soc., Ser. A, 219, 186-203.

[79] Taylor, G.I., 1954. The dispersion of matter in turbulent flow through a pipe. Proc. Roy. Soc., Ser. A, $223,446-468$.

[80] Modeling fracture propagation from a wellbore and heat extraction from a fracture in HDR. Progress Report NDRM-00-02. Submitted to Department of Energy, 2000.

[81] Lowell, R.P., 1976. Comments on 'Theory of heat extraction from fracture hot dry rock' by A.C. Gringarten, P.A. Witherspoon, and Y. Ohnishi. J. Geophys. Res., 81, 359-360.

[82] Cheng, A.H.-D., Sidauruk, P. \& Abousleiman, Y. 1994., Approximate inversion of the Laplace transform. Mathematica J., 4, 76-82.

[83] Polyanin, A.D. \& Manzhirov, A.V., 1998. Handbook of Integral Equations, CRC Press.

[84] Press, W.H., Teukolsky, S.A., Vetterling, W.T. \& Flannery, B.P., 1992. Numerical Recipe in Fortran, the Art of Scientific Computing, 2nd ed., Cambridge Univ. Press.

[85] Gringarten, A.C., Witherspoon, P.A. \& Ohnishi, Y., 1974. Theory of heat extraction from fracture hot dry rock. J. Geophys. Res., 80, 1120-1124. 
[86] Kolditz, O., 1995. Modelling flow and heat transfer in fractured rocks: Dimensional effect of matrix hear diffusion. Geothermics, 24, 421-437.

[87] Gringarten, A.C. \& Sauty, J.P., 1975. A theoretical study of heat extraction from aquifers with uniform regional flow," J. Geophys. Res., 80, 4956-4962.

[88] Mogilevskaya, S.G., 2000. Modeling two-dimensional fracture propagation using a complex variable displacement discontinuity method. Report to the Department of Geology \& Geological Engineering, University of North Dakota.

[89] NDRM-00-02. Modeling fracture propagation from a wellbore \& heat extraction from a fracture in HDR. Quarterly Report Submitted to DOE by Dept. of Geology \& Geological Engineering, University of North Dakota, 2000.

[90] NDRM-00-03. Numerical analysis of hydraulic fracture propagation under a thermoelastic perturbation of the state of stress \& A two-dimensional solution for heat extraction from a fracture in hot dry rock. Quarterly Report Submitted to DOE by Dept. of Geology \& Geological Engineering, University of North Dakota, 2000.

[91] NDRM-00-04. User interface development \& Three-dimensional heat extraction from a fracture in HDR. Quarterly Report Submitted to DOE by Dept. of Geology \& Geological Engineering, University of North Dakota, 2000.

[92] Koshelev, V. F. \& Linkov, A.M., 1999. Complex BEM in periodic and double-periodic elasticity problems. Proc. 13th ASCE Engrg. Mech. Conf., CDROM.

[93] Linkov, A. M. \& Mogilevskaya, S. G., 1994. Complex hypersingular integrals and integral equations in plane elasticity. Acta Mechanica, 105, 189-205.

[94] Almaguer, J., Manrique, J., Wickramasuriya, S., Habbtar, A., de Cardenas, J., May, D., McNally, A.C. \& Sulbaran, A., 2002. Orienting perforations in the right direction. Oil Filed Review, Spring 2002, 16-31.

[95] van As, A. \& Jeffrey, R.G., 2002. Hydraulic fracture growth in naturally fractured rock: Mine through, mapping, and analysis. NARMS-TAC 2003, Toronto, Canada.

[96] Linkov, A.M. \& Koshelev V.F., 1999. Complex variable BIE and BEM for a plane doubly periodic system of flaws. J. Chinese Inst. Engineers, 22(6), 709-720.

[97] Linkov, A.M. \& Mogilevskaya, S., 1991. Complex hypersingular integrals and integral equations in plane problems of elasticity theory. In: Researches on Structure Mechanics and Materials. Leningrad: Leningrad. Inst. for Build. Eng., 17-34 (in Russian).

[98] Linkov, A.M., \& Mogilevskaya, S., 1995. On the theory of complex hypersingular equations. In: Atluri, S., Yagawa, G. \& Cruse, T. (ed.): Computational Mech.'95(2), Berlin - Heidelberg-New-York: SpringerVerlag, 2836-2840.

[99] Savitski, A., 2001. Calculation of thermal stress due to heat extraction from HDR. Report to the Dept. of Geology \& Geological Engineering, University of North Dakota. 
[100] Mossop, A. \& Segall, P., 2001. Induced seismicity in geothermal field. I- A thermoelastic injection model (on the Web; to be published).

[101] Dobroskok, A. A., 2001. On a new method for iterative calculation of crack trajectory. Int. J. Fracture, 111, L41-46.

[102] Linkov, A.M., 1983. Plane problems of the static loading of a piecewise homogeneous linearly elastic medium. J. Appl. Math. and Mech., 47, 527-532.

[103] Linkov, A.M., 2002. Boundary Integral Equations in Elasticity Theory. Dordrecht: Kluwer Academic Publishers. 286 p.

[104] Hayashi, K., Willis-Richards, J., Hopkirk, R.J. \& Niibori, Y., 1999. Numerical models of HDR geothermal reservoirs - a review of current thinking and progress. Geothermics, 28, 507-518.

[105] Willis-Richards, J. \& Wallroth, T., 1995. Approaches to the modeling of HDR reservoirs: a review. Geothermics, 24, 307-332.

[106] Kolditz, O. \& Clauser, C., 1998. Numerical simulation of flow and heat transfer in fractured crystalline rocks: Application to the hot dry rock site in Rosemanowes (U.K.). Geothermics, 27, 1-23.

[107] Kohl, T., Evans, K.F., Hopkirk, R.J. \& Ryback, L. 1995., Coupled hydraulic, thermal, and mechanical considerations for the simulation of hot dry rock reservoirs. Geothermics, 24, 345-359.

[108] Cheng, A.-D., Ghassemi, A. \& Detournay, E., 2001. A two-dimensional solution for heat extraction from a fracture in hot dry rock. Int. J. Numer. Analy. Meth. Geomech., 25, 1327-1338.

[109] Abe, H., Mura, T. \& Keer, L.M., 1976. Heat extraction from a hydraulically fractured penny-shaped crack in hot dry rock. Proc. 2nd Workshop Geothermal Reservoir Engineering, Stanford University, CA, 200-212.

[110] Bodvarsson, G. \& Tsang, C.F., 1982. Injection and thermal breakthrough in fractured geothermal reservoirs. J. Geophys. Res., 87, 1031-1048.

[111] Heuer, N., Kupper, T. \& Windelberg, D., 1991. Mathematical model of a hot dry rock system. Geophys. J. Int., 105, 659-664.

[112] Kruger, P., Dyadkin, Y.D., Gendler, S., Artemieva, E. \& Smirnova, N., 1991. Comparison of thermal cooldown estimates in the Russkie Komarovtsy petrogeothermal reservoir. Proc. 16th Workshop Geothermal Reservoir Engineering, Stanford University, CA, 159-164.

[113] Stehfest, H., 1989. Numerical inversion of Laplace transforms. Comm. ACM, 13, 47-49 and 624, 1970.

[114] Strack, O. D.L., Groundwater Mechanics, Prentice Hall, New Jersy, 732 p.

[115] Rodemann, H., 1982. Analytical model calculations on heat exchange in a fracture. In: Urach Geothermal Project, Haenel, R. (ed.), Stuttgart, 351-353.

[116] Ligget, A.J. \& Liu, P.L-F., 1983. The Boundary Integral Equation Methods for Porous Media Flow, George Allen \& Unwin, 255 p. 
[117] Aadnoy, B.S. \& Angell-Olsen, F., 1995. Some effects of ellipticity on the fracturing and collapse behavior of a borehole. Int. J. Rock. Mech. Mining Sci., 32(6), 621-627.

[118] Berchenko, I., 1998. Thermal Loading of Saturated Rock Mass: Field Experiment and Modeling Using Thermoporoelastic Singular Solutions. Ph.D. Dissertation, University of Minnesota.

[119] Biot, M.A., 1941. General theory of three-dimensional Consolidation. J. Appl. Phys., 12, 155-164.

[120] Cheng, A.H.-D. \& Predeleanu, M., 1987. Transient boundary element formulation for linear poroelasticity. Appl. Math. Modeling. 11, 285-290.

[121] Cheng, A.H-D. \& Detournay, E., 1998. On singular integral equations and fundamental solutions of poroelasticity. Int. J. Solids \& Structures 35(34/35), 4521-4555.

[122] Curran, J.H. \& Carvalho, J.L., 1987. A displacement discontinuity model for fluid-saturated porous media. Proc. 6th Congress of the I.S.R.M. (I), Montreal, 73-78.

[123] Detournay, E. \& Cheng, A.H.-D., 1993. Fundamentals of poroelasticity. In J.A. Hudson (ed.) Comprehensive Rock Engineering: Principles, Practices, and Projects (2), 113-171, Pergamon Press.

[124] Ghassemi, A., Cheng, A. H.-D., Diek, A. \& Roegiers, J.-C. 2001. A complete plane- strain fictitious stress boundary element method for poroelastic media. J. Eng. Anal. Boundary Elements, 25(1), 41-48.

[125] Ghassemi, A. \& Zhang, Q., 2003. Indirect Boundary element Methods for plane strain Porothermoelasticity J. Eng. Anal. Boundary Elements (submitted).

[126] McTigue, D.F., 1986. Thermoelastic response of fluid-saturated porous Rock. J. Geophys. Res., 91(B9), 9533-9542.

[127] Palciauskas V.V. \& Domenico P.A., 1982. Characterization of drained and undrained response of thermally loaded repository rocks. Water Resour. Res., 18, 281-290.

[128] Smith, D.W. \& Booker, J.R., 1993. Boundary element analysis of linear thermoelastic consolidation. Int. J. Num. Anal. Methods. Geomech., 20, 457-488.

[129] van As, A. \& Jeffrey, R.G., 2002. Hydraulic fracture growth in naturally fractured rock: Mine through, mapping, and analysis. NARMS-TAC 2003, Toronto, Canada.

[130] Crouch S.L. \& Starfield A.M., 1983. Boundary Element Method in Solid Mechanics, George Allen \& Unwin, London.

[131] Savruk, M.P., Osiv, P.N. \& Prokopchuk, I.V., 1989. Numerical analysis in plane problems of crack theory. Kiev: Naukova Dumka. 248 p. (In Russian).

[132] Cotterell, B. \& Rice, J.R., 1980. Slightly curved or kinked cracks. Int. J. Fracture, 16, 155-168.

[133] Radon, J.C., Leevers P.S. \& Culver, L.E., 1977. Fracture toughness of PMMA under biaxial stress. Fracture, 8, 1113-1116. 
[134] Volkov, V. (ed.), 1987. Approximation of the compound curves by the arcs of the circles and its application to the problems of mechanical engineering. Kemerovo: Kuzbass Technical University. (in Russian).

[135] Sherburn, S., 1984. Seismic monitoring during a cold water injection experiment, Wairakei Geothermal Field: preliminary results, In: Proc. 6th New Zealand Geothermal Workshop, 6, 129-133.

[136] Stark, M.A., 1990. Imaging injected water in the Geysers reservoir using microearthquakes data, In: Geothermal Resources Council Trans. 14(II), 1697-1704.

[137] Koshelev, V. and Ghassemi, A. 2003. Complex variable BEM for thermo- and poroelasticity. J. Eng. Anal. Boundary Elements (in review). 


\section{Appendix A}

\subsection{Mathematical Formulation and Numerical Aspects of GeoFrac}

\subsubsection{Basic integral equation for a plane without a circular hole}

Details of the building block of the current version (1.0) of GeoFrac are described in this section. The BEM under consideration is the most general way to numerically solve the HCV-BIE. The equation proposed by Linkov and Mogilevskaya [5] is:

$$
\frac{1}{2 \pi i} \int_{S}\left\{\begin{array}{c}
\frac{2 \Delta u}{(\tau-t)^{2}} d \tau-\Delta u \frac{\partial}{\partial t} d k_{1}-\Delta \bar{u} \frac{\partial}{\partial t} d k_{2}+\left(2 a_{1}-a_{3}\right) \frac{\sigma}{\tau-t} d \tau+ \\
\left(a_{1}-a_{3}\right) \sigma \frac{\partial k_{1}}{\partial t} d \tau+a_{1} \bar{\sigma} \frac{\partial k_{2}}{\partial t} d \bar{\tau}
\end{array}\right\}=\frac{1}{2} a_{2} \sigma(t)+s^{\infty}
$$

where $S$ is the total boundary of the system of blocks. The contact between surfaces of adjacent blocks is accounted as a single line at which both mechanical properties and field values may experience discontinuities; $\Delta u(\tau)$ is the complex displacement discontinuity in the global coordinates : $\Delta u=u^{+}-u^{-}=\Delta u_{x}+i \Delta u_{y}$; $\sigma(t)$ is the traction vector at a point $t: \sigma(t)=\sigma_{n n}+i \sigma_{n t} ;(n, t)$ is a local coordinates; overbar denotes a complex conjugation;

$$
a_{1}=\frac{1}{2 \mu^{+}}-\frac{1}{2 \mu^{-}} ; a_{2}=\frac{\kappa^{+}+1}{2 \mu^{+}}+\frac{\kappa^{-}+1}{2 \mu^{-}} ; a_{3}=\frac{\kappa^{+}+1}{2 \mu^{+}}-\frac{\kappa^{-}+1}{2 \mu^{-}}
$$

the sign "plus" denotes the limit value from the left side of the travelling path, the sign "minus" refers to the right side of the travelling path, $\kappa=3-4 \nu$ for plane strain; $\kappa=\frac{3-\nu}{1+\nu}$ for plane stress; $\nu$ is Poisson's ratio; $\mu$ is shear modulus;

$$
s^{\infty}=-\frac{\kappa_{0}+1}{4 \mu_{0}}\left[\sigma_{x}^{\infty}+\sigma_{y}^{\infty}+e^{-2 i \alpha_{t}}\left(\sigma_{y}^{\infty}-\sigma_{x}^{\infty}-2 i \sigma_{x y}^{\infty}\right)\right]
$$

$\sigma_{x}^{\infty}, \sigma_{y}^{\infty}, \sigma_{x y}^{\infty}$ are stresses at infinity; $\alpha_{t}$ is the angle between $x$-axis and the tangent vector at a point $t$; subscript 0 relates to the external domain embedding the system of blocks; $k_{1}$ and $k_{2}$ are the conventional kernels in the Complex Variables BIE:

$$
k_{1}(\tau, t)=\ln \frac{\tau-t}{\bar{\tau}-\bar{t}} ; k_{2}(\tau, t)=\frac{\tau-t}{\bar{\tau}-\bar{t}}
$$

It should be noted that another form of hypersingular BIE formulation was implemented in [76] that is based on the fundamental solution for a plane with a circular hole. Such a method has the advantage that discretization of the circular wellbore boundary is not necessary. The integral equation for this approach is presented next. Both approaches use similar numerical schemes for fracture propagation.

\subsubsection{Basic integral equation for a plane with a circular hole}

The CHSIE for isotropic elastic plane with a circular opening and an arbitrary curvilinear crack is given by [76]:

$$
\frac{1}{2 \pi i}\left\{2 \int_{S} \frac{\Delta u d \tau}{(\tau-t)^{2}}-\int_{S} \Delta u \frac{\partial}{\partial t}\left[d \ln \frac{\tau-t}{\bar{\tau}-\bar{t}}+d \ln \left(\bar{\tau}-\frac{R^{2}}{t}\right)+d \ln \left(\tau-\frac{R^{2}}{\bar{t}}\right)-\right.\right.
$$




$$
\begin{gathered}
\left.\left(t-\frac{R^{2}}{\bar{t}}\right)\left[\frac{R^{2}}{\bar{t}^{2}} d \frac{\bar{\tau}-\bar{t}}{\left(\tau-\frac{R^{2}}{\bar{t}}\right)^{2}}-\frac{d \tau}{\left(\tau-\frac{R^{2}}{\bar{t}}\right)^{2}}+\frac{d \tau}{\tau^{2}}\right)\right]+ \\
\int_{S} \overline{\Delta u} \frac{\partial}{\partial t}\left[-d \frac{\tau-t}{\bar{\tau}-\bar{t}}+d \frac{\tau-t}{\bar{\tau}-\frac{R^{2}}{t}}-d \frac{\tau-t}{\bar{\tau}}+\frac{R^{2}}{\bar{t}} \frac{d \bar{\tau}}{\bar{\tau}^{2}}-\right. \\
\left.\left.\left(t-\frac{R^{2}}{\bar{t}}\right) \frac{R^{2}}{\bar{t}^{2}} \frac{d \tau}{\left(\tau-\frac{R^{2}}{\bar{t}}\right)^{2}}\right]\right\}=\frac{1+\kappa}{2 \mu}\left(\sigma(t)+\frac{d \bar{t}}{d t} \frac{p R^{2}}{\bar{z}^{2}}\right)+\sigma^{\infty}(t)
\end{gathered}
$$

where $S$ is the crack boundary; $\sigma=\sigma_{n}+i \sigma_{s}$, where $\sigma_{n}$ and $\sigma_{s}$ are the normal and shear traction on $S$, respectively; $i=\sqrt{-1}$. The unit outward normal $n$ is directed to the right of the direction of travel on the boundary; $\Delta u=u^{+}-u^{-}$is the DD in the global Cartesian coordinate system with $u^{+}$and $u^{-}$representing the limit values of displacements from the left or right sides of the contours, respectively. $p$ is normal pressure on the contour of the opening, $t=t_{1}+i t_{2} \in S$, and $\sigma^{\infty}(t)$ is defined by

$$
\begin{aligned}
\sigma^{\infty}(t)= & -\frac{\kappa+1}{2 \mu}\left[\frac{\sigma_{h}+\sigma_{H}}{2}\left(1+\frac{R^{2}}{\bar{t}^{2}} \frac{d \bar{t}}{d t}\right)+\frac{\sigma_{H}-\sigma_{h}}{2}\left(\frac{R^{2}}{t^{2}}+\right.\right. \\
& \left.\left.\frac{d \bar{t}}{d t}+\frac{R^{2}}{\bar{t}^{2}}-\frac{2 R^{2} t}{\bar{t}^{3}} \frac{d \bar{t}}{d t}+\frac{3 R^{4}}{\bar{t}^{4}} \frac{d \bar{t}}{d t}\right)\right]
\end{aligned}
$$

in which $\kappa=3-4 \nu, \nu$ is the Poisson's ratio, $\mu$ is the shear module and a bar over a symbol denotes complex conjugation.

It can be observed that the boundary of the opening is not involved in (191) because this equation was obtained using a Green's function that automatically satisfies the pressure condition on the opening boundary. This means that the boundary of the opening need not to be discretized for numerical solution of (191).

The SIFs at the crack tip $z_{1}$ can be calculated from the known DD according to [68]:

$$
\left(K_{1}-i K_{2}\right)_{z_{1}}=-\frac{\sqrt{2 \pi} \mu i}{\kappa+1} \exp (-i \psi / 2) \lim _{\tau \rightarrow z_{1}}\left(\frac{\Delta u}{\sqrt{z_{1}-\tau}}\right), \tau \in S
$$

where $\psi$ is the angle between the tangent at the tip $z_{1}$ and axis $O x$. Note that computed SIFs are independent on Poisson's ratio as can be seen from the structure of (191).

The numerical technique for solving equations (191) and (187), and calculating the SIFs is based on the complex variables BEM with straight and circular elements. The formulation is similar to the one developed for piece-wise homogeneous plane [68]-[72] and half-plane [70]. As mentioned above, the boundary of the opening need not be discretized, however, the boundary $S$ is divided on $N$ equal straight or circular elements $S_{j}$. The DDs $\Delta u$ are approximated by complex second degree Lagrange polynomials along each element $S_{j}$. For the tip element a square root asymptotics for $\Delta u$ is used.

Thus, for each element $S_{j}$ the following interpolation is used:

$$
\Delta u^{j}(\tau) \approx \sum_{\ell=1}^{3} \varpi_{\ell}^{j}(\tau) \Delta u_{\ell}^{j} Q_{\ell}^{j}(\tau)
$$


where

$$
Q_{\ell}^{j}(\tau)=\prod_{\substack{s \neq \ell \\
s=1}}^{3} \frac{\tau-\tau_{s}^{j}}{\tau_{\ell}^{j}-\tau_{s}^{j}} ; \varpi_{\ell}^{j}(\tau)=\left\{\begin{array}{cc}
\frac{1}{\sqrt{z_{1}-\tau}} & \text { for ordinary elements } \\
\sqrt{z_{1}-\tau_{\ell}^{j}} & \text { for the tip } z_{1}
\end{array}\right.
$$

$\Delta u_{\ell}^{j}$ are the values of $\Delta u(\tau)$ in the nodes $\tau_{\ell}^{j}(\ell=1, \ldots, 3)$ of $j$-th element.

By substituting (193) into (191) and applying a collocation method, a system of linear algebraic equations is obtained. Upon solution of this system, the SIFs are found form the DDs using:

$$
\left(K_{1}-i K_{2}\right)_{z_{1}}=-\frac{\sqrt{2 \pi} \mu i}{\kappa+1} \exp (-i \psi / 2) \sum_{\ell=1}^{3} \frac{\Delta u_{\ell}^{q} Q_{\ell}^{q}\left(z_{1}\right)}{\sqrt{z_{1}-\tau_{\ell}^{q}}}
$$

where $q$ is the number of the element containing the tip $z_{1}$.

The numerical algorithm for two-dimensional smooth crack growth can be summarized as follows:

1. numerical calculation of the DD along the initial crack and the stress intensity factors (SIFs) $K_{1}$ and $K_{2}$

2. definition of the deviation angle $\theta$;

3. smooth approximation of the new crack path by a circular arc or a straight segment.

Each of these aspects are described in detail below.

\subsubsection{Calculation of SIFs}

Analytic method To calculate SIFs for a curvilinear crack $L$ the following formulae is used as suggested in [68]:

$$
\begin{aligned}
& \left(K_{1}-i K_{2}\right)_{z_{1}}=-\frac{\sqrt{2 \pi} \mu i}{\kappa+1} \exp (-i \varphi / 2) \lim _{\tau \rightarrow z_{1}}\left(\frac{\Delta u}{\sqrt{\tau-z_{1}}}\right) \\
& \left(K_{1}-i K_{2}\right)_{z_{2}}=-\frac{\sqrt{2 \pi} \mu i}{\kappa+1} \exp (-i \psi / 2) \lim _{\tau \rightarrow z_{2}}\left(\frac{\Delta u}{\sqrt{z_{2}-\tau}}\right)
\end{aligned}
$$

where $\varphi$ is the angle between the tangent in the left crack tip, $z_{1}$, and axis $O x ; \psi$ is the similar angle in the right tip $z_{2} ; i=\sqrt{-1} ; \Delta u=u^{+}-u^{-}$is the displacement discontinuity (DD) in global coordinate system; $u^{+}$and $u^{-}$are the limit values of the displacements when approaching the crack boundary from its left or right side, respectively; $\kappa=3-4 \nu$ in plane strain; $\kappa=(3-\nu) /(1+\nu)$ in plane stress; $\mu=\mu^{+}=\mu^{-}$is the shear module; $\nu=\nu^{+}=\nu^{-}$is the Poisson's ratio; the signs "+" and "-" denote the elastic constants for the region that is to the left and right of the direction of traverse on $L ; \tau \in L$.

Suppose that the crack $L$ is situated in a piecewise homogeneous plane. The values of the DD involved in (195) can be found from the solution of CHSIE for this problem. This was obtained by Linkov and Mogilevskaya [97] through direct differentiation of the corresponding complex singular equation obtained by Linkov [102]. It can be written as: 


$$
\begin{gathered}
\frac{1}{\pi i} \int_{S} \frac{\Delta u(\tau)}{(\tau-t)^{2}} d \tau+\frac{1}{2 \pi i} \int_{S} \Delta u(\tau) S_{1}(\tau, t) d \tau-\frac{1}{2 \pi i} \int_{S} \overline{\Delta u(\tau)} S_{2}(\tau, t) d \bar{\tau}+ \\
+\frac{1}{\pi i} \int_{S}\left(a_{1}-\frac{a_{3}}{2}\right) \frac{\sigma(\tau)}{\tau-t} d \tau+\frac{1}{2 \pi i} \int_{S}\left(a_{1}-a_{3}\right) \sigma(\tau) S_{3}(\tau, t) d \tau- \\
-\frac{1}{2 \pi i} \int_{S} a_{1} \overline{\sigma(\tau)} S_{4}(\tau, t) d \bar{\tau}-\frac{a_{2}}{2} \sigma(t)=f(t), t \in S
\end{gathered}
$$

where $S$ is the totality of external and internal boundaries including the crack boundary $L ; \sigma=\sigma_{n}+i \sigma_{\tau}$; $\sigma_{n}$ is a normal and $\sigma_{\tau}$ is a shear traction on $S$. The unit normal, $n$, is directed to the right of the direction of traverse; the direction of traverse is arbitrary for contact boundaries, however, it is counter clockwise for an external boundary. Note that a bar over a symbol denotes complex conjugation and $a$ 's are given by:

$$
\begin{gathered}
a_{1}=\frac{1}{2 \mu^{+}}-\frac{1}{2 \mu^{-}} ; a_{2}=\frac{1+\kappa^{+}}{2 \mu^{+}}+\frac{1+\kappa^{-}}{2 \mu^{-}} ; a_{3}=\frac{1+\kappa^{+}}{2 \mu^{+}}-\frac{1+\kappa^{-}}{2 \mu^{-}} ; \\
f(t)=-\frac{\kappa_{\infty}+1}{4 \mu_{\infty}}\left[\sigma_{x x}^{\infty}+\sigma_{y y}^{\infty}+\frac{d \bar{t}}{d t}\left(\sigma_{y y}^{\infty}-\sigma_{x x}^{\infty}-2 i \sigma_{x y}^{\infty}\right)\right]
\end{gathered}
$$

$\sigma_{x x}^{\infty}, \sigma_{y y}^{\infty}, \sigma_{x y}^{\infty}$ are the stresses at infinity (for a finite region these stresses are equal to zero); $t=x+i y$;

$$
\begin{gathered}
S_{1}(\tau, t)=-\frac{\partial^{2}}{\partial \tau \partial t} \ln \frac{\tau-t}{\bar{\tau}-\bar{t}}=\frac{d \bar{t}}{d t} \frac{d \bar{\tau}}{d \bar{\tau}} \frac{1}{(\bar{\tau}-\bar{t})^{2}}-\frac{1}{(\tau-t)^{2}} \\
S_{2}(\tau, t)=\frac{\partial^{2}}{\partial \bar{\tau} \partial t} \frac{\tau-t}{\bar{\tau}-\bar{t}}=\left(1+\frac{d \bar{t}}{d t} \frac{d \tau}{d \bar{\tau}}\right) \frac{1}{(\bar{\tau}-\bar{t})^{2}}-2 \frac{d \bar{t}}{d t} \frac{\tau-t}{(\bar{\tau}-\bar{t})^{3}} \\
S_{3}(\tau, t)=\frac{\partial}{\partial t} \ln \frac{\tau-t}{\bar{\tau}-\bar{t}}=\frac{d \bar{t}}{d t} \frac{1}{\bar{\tau}-\bar{t}}-\frac{1}{\tau-t} \\
S_{4}(\tau, t)=-\frac{\partial}{\partial t} \frac{\tau-t}{\bar{\tau}-\bar{t}}=\frac{1}{\bar{\tau}-\bar{t}}-\frac{d \bar{t}}{d t} \frac{\tau-t}{(\bar{\tau}-\bar{t})^{2}}
\end{gathered}
$$

with $\frac{1}{2 \mu^{-}}=0, u^{-}=0$ for a plane with an inclusion; and $\frac{1}{2 \mu^{+}}=0, u^{+}=0$ for a plane with a hole.

Boundary and contact conditions may be different on different parts of $S-L$ and may include prescribed tractions, DD's, or prescribed relationships between tractions and DD's.

The CHSIE (196) have been proven to have zero index in the functional class of N by Muskhelishvili [98]. This means there no additional conditions needed to be satisfied.

Numerical procedure Equation (196) can be solved using a boundary element method. As it was shown in [68] only two types of the boundary elements (straight segments and circular arcs) need to be employed to represent any smooth part of the boundary. We represent the contour $S$ by a totality of such boundary elements $S_{j}, j=1, \ldots, n$.

Because of the continuity constraints $\left(C^{1}\right.$ continuity is needed for $\left.\Delta u\right)$ we will use nonconforming elements, when the collocation points are away from the edge ones. Furthermore, the use of conforming elements has 
been shown not to improve accuracy dramatically. The "quality" of the approximations is more important than the location of the nodes.

The tractions $\sigma$ and DD's, $\Delta u$, are approximated by complex Lagrange polynomials of arbitrary degree $m-1$ at the each element $S_{j}$. For the tip elements (in a crack problem) the asymptotics for $\Delta u$ are taken into account. Then for the element $S_{j}$ one has:

$$
\begin{gathered}
\Delta u^{j}(\tau) \approx \sum_{\ell=1}^{m} \Delta u_{\ell}^{j} P_{\ell}^{j}(\tau) \\
\sigma^{j}(\tau) \approx \sum_{\ell=1}^{m} \sigma_{\ell}^{j} Q_{\ell}^{j}(\tau)
\end{gathered}
$$

where

$$
Q_{\ell}^{j}(\tau)=\prod_{s \neq \ell}^{m} \frac{\tau-\tau_{s}^{j}}{\tau_{\ell}^{j}-\tau_{s}^{j}}
$$

and

$$
P_{\ell}^{j}(\tau)=\left\{\begin{array}{cc}
Q_{\ell}^{j}(\tau), & \text { for ordinary elements } \\
\frac{\sqrt{\tau-a^{j}}}{\sqrt{\tau_{\ell}^{j}-a^{j}}} Q_{\ell}^{j}(\tau), & \text { for the left tip } a^{j} \\
\frac{\sqrt{b^{j}-\tau}}{\sqrt{b^{j}-\tau_{\ell}^{j}}} Q_{\ell}^{j}(\tau), & \text { for the right tip } b^{j}
\end{array}\right.
$$

$\Delta u_{\ell}^{j}, \sigma_{\ell}^{j}$ are the values of $\Delta u(\tau)$ and $\sigma(\tau)$ in the nodes $\tau_{\ell}^{j}(\ell=1, \ldots, m)$ of $j$-th element.

On substituting (198),(199) into (196) a system of equations is obtained:

$$
\left\{\begin{array}{c}
\sum_{j=1}^{n} \sum_{\ell=1}^{m}\left[\alpha_{\ell j}(t) \Delta u_{\ell}^{j}+\beta_{\ell j}(t) \overline{\Delta u_{\ell}^{j}}+\gamma_{\ell j}(t) \sigma_{\ell}^{j}+\zeta_{\ell j}(t) \overline{\sigma_{\ell}^{j}}\right]- \\
-a_{2} \pi i \sigma(t) \approx 2 \pi i f(t)
\end{array}\right.
$$

where:

$$
\begin{gathered}
\alpha_{\ell j}(t)=2 \int_{L_{j}} \frac{P_{\ell}^{j}(\tau) d \tau}{(\tau-t)^{2}}+\int_{L_{j}} P_{\ell}^{j}(\tau) S_{1}(\tau, t) d \tau \\
\beta_{\ell j}(t)=-\int_{L_{j}} \overline{P_{\ell}^{j}(\tau)} S_{2}(\tau, t) d \bar{\tau} ; \zeta_{\ell j}(t)=-\int_{L_{j}} a_{1} \overline{Q_{\ell}^{j}(\tau)} S_{4}(\tau, t) d \bar{\tau} \\
\gamma_{\ell j}(t)=\int_{L_{j}}\left(2 a_{1}-a_{3}\right) \frac{Q_{\ell}^{j}(\tau) d \tau}{\tau-t}+\int_{L_{j}}\left(a_{1}-a_{3}\right) Q_{\ell}^{j}(\tau) S_{3}(\tau, t) d \tau
\end{gathered}
$$

Equations (200) can be solved by using a collocation method. To do so, it is suppose that the collocation points $t_{1}, \ldots t_{m \times n}$ coincide with the nodes $\tau_{1}^{1}, \tau_{2}^{1}, \ldots, \tau_{m}^{1}, \tau_{1}^{2}, \ldots \tau_{m}^{2}, \ldots, \tau_{1}^{n}, \ldots \tau_{m}^{n}$ of the Lagrange polynomials. 


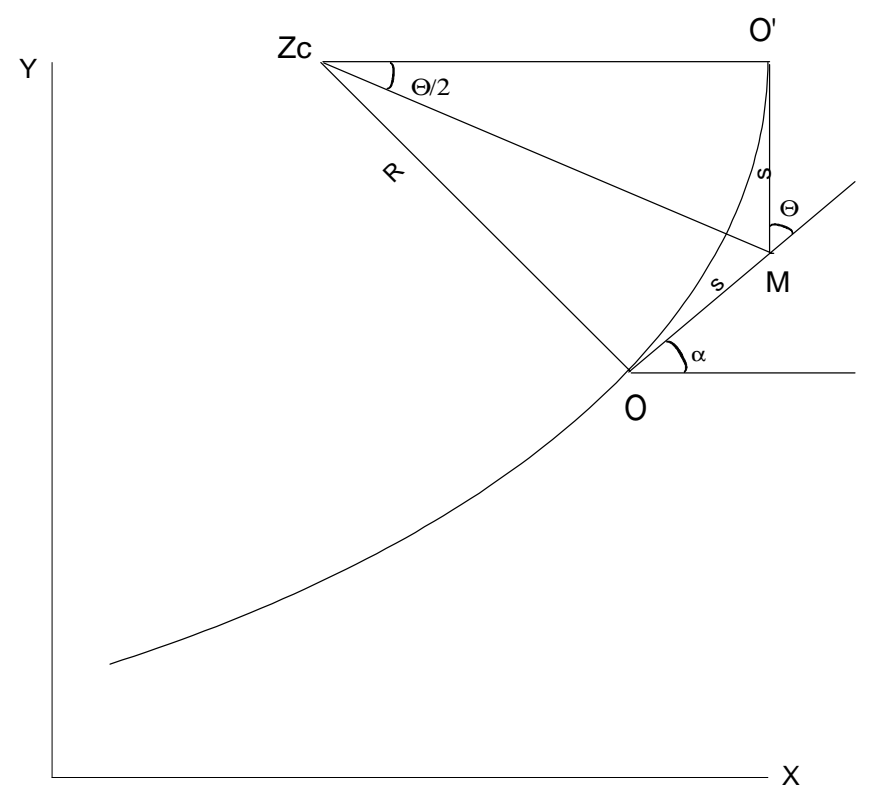

Figure 94: Approximation of the crack path at each propagation stage

Using (195) and (198), the SIFs at the left tip (element $S_{p}, z_{1}=a^{p}$ ) and at the right tip (element $S_{r}$, $\left.z_{2}=b^{r}\right)$ can be evaluated by:

$$
\begin{aligned}
& \left(K_{1}-i K_{2}\right)_{a^{p}}=-\frac{\sqrt{2 \pi} \mu i}{\kappa+1} \exp (-i \varphi / 2) \sum_{\ell=1}^{3} \frac{\Delta u_{\ell}^{p} Q_{\ell}^{p}\left(a^{p}\right)}{\sqrt{\tau_{\ell}^{p}-a^{p}}} \\
& \left(K_{1}-i K_{2}\right)_{b^{r}}=-\frac{\sqrt{2 \pi} \mu i}{\kappa+1} \exp (-i \psi / 2) \sum_{\ell=1}^{3} \frac{\Delta u_{\ell}^{r} Q_{\ell}^{r}\left(b^{r}\right)}{\sqrt{b^{r}-\tau_{\ell}^{r}}}
\end{aligned}
$$

All the integrals involved in (201) are calculated in close-form for both types of the boundary elements employed. The expression to evaluate them are given in [68]. The accuracy of the suggested method depends only on the "quality" of the approximation of the unknown function and the boundary of the curve. Approximation of the DD's by polynomials of the second degree provides the best results [68] if the boundary elements situated close to the crack tip have approximately equal lengths.

\subsubsection{Approximation of the crack path at each stage of propagation}

The following new scheme is suggested for representing each portion of the crack path. Consider the crack tip at $O$, known from the previous stage of the propagation, i.e., $O$ is the right tip of an initial crack. Suppose the angle between the tangent at point $O$ and the $x$-axis is also known from the previous stage and is equal to $\alpha$. The tangent at point $O$ is continued till a point $M$ (Figure 94) so that the distance between $O$ and $M$ will be equal to some value $s$. Assume the new path direction, $\theta$, is known from a crack propagation criterion. From point $M$ a straight line is drawn at an angle $\theta$ to the direction of $O M$. On this line the point $O^{\prime}$ is defined in such a way that the distance between $O$ and $O^{\prime}$ is equal to $s$ (Figure 1). Then, the 
crack path is represented by the circular arc which links points $O$ and $O^{\prime}$ such that lines $O M$ and $O^{\prime} M$ are the tangents to the arc at points $O$ and $O^{\prime}$, respectively. If $\theta=0$, then $O^{\prime} \equiv M$ and the straight segment $O M$ will represent the crack path. The only unknown is the magnitude of distance $s$. The value of $s$ can be found from the following condition:

$$
s=\frac{\ell}{\theta} \tan \frac{\theta}{2}
$$

where $\ell$ is the length of the right tip boundary element from the previous stage of the propagation. Equation (203) expresses the fact that the length of the circular arc is equal to $\ell$. As it was mentioned earlier, the elements close to the crack tip need to have approximately the same lengths.

The coordinates of the current tip, $O^{\prime}\left(x_{1}, y_{1}\right)$, can be found from the following formulae:

if $\theta \neq 0$, then

$$
\begin{aligned}
& x_{1}=x+s[\cos \alpha+\cos (\alpha+\theta)] \\
& y_{1}=y+s[\sin \alpha+\sin (\alpha+\theta)]
\end{aligned}
$$

if $\theta=0$, then

$$
\begin{aligned}
& x_{1}=x+s \cos \alpha \\
& y_{1}=y+s \sin \alpha
\end{aligned}
$$

where $x, y$ are the coordinates of the previous tip $O$.

Therefore, the coordinates of two points of the circle, the angle $\alpha$ between the $O x$ axis, and the tangent at point $O$ are known. The coordinates of the center of the circle, $z_{c}\left(x_{c}, y_{c}\right)$, which links $O$ and $O^{\prime}$ can be found from the solution of the corresponding problem of analytical geometry (see e.g., [134]). They take a form

$$
\begin{aligned}
& x_{c}=x+T \sin \alpha \\
& y_{c}=y-T \cos \alpha
\end{aligned},
$$

where

$$
T=\frac{\left(y-y_{1}\right)^{2}+\left(x-x_{1}\right)^{2}}{2\left[\left(y-y_{1}\right) \cos \alpha-\left(x-x_{1}\right) \sin \alpha\right]}
$$

Definition of the deviation's angle $\theta$ As referenced previously, two crack propagation criteria are used to define $\theta$. In the first stage, the maximum tensile stress criterion is used. It follows from this criterion that crack propagates in the direction perpendicular to the direction of greatest tension when the stress $\sigma_{\theta \theta}$ (in cylindrical coordinate system with the origin in the crack tip) reaches a critical value. This gives the following expression for the deviation angle $\theta$ :

$$
\theta=2 \arctan \frac{K_{1} \pm \sqrt{K_{1}^{2}+8 K_{2}^{2}}}{4 K_{2}}
$$

where $K_{1}, K_{2}$ are the SIFs for the right tip of the initial crack. The sign in (207) is chosen so as to maximize the stress component $\sigma_{\theta \theta}$ defined by: 


$$
\sigma_{\theta \theta}=\frac{1}{\sqrt{2 \pi r}} \cos \frac{\theta}{2}\left[K_{1} \cos ^{2} \frac{\theta}{2}-\frac{3}{2} K_{2} \sin \theta\right]
$$

For the next propagation stages, the fact that $K_{2}=0$ at the current crack tip is utilized. To obtain $\theta$ from this condition one needs to follow an iterative procedure for the numerical solution of the equation $K_{2}=0$. In the first iteration step one finds $\theta=\theta_{1}$ from equation (207). The next iteration steps, $\theta=\theta_{n}$ $(n=2, \ldots)$ involve the following sequence of operations:

1. define the crack tip $O\left(\theta_{n-1}\right)$ as it was described above. The value of $\ell$ from formula (203) is usually supposed to equal 0.1 measured from the end of the initial crack;

2. solve equation (196) numerically; and

3. calculate $K_{2}\left(\theta_{n-1}\right)$ at $O\left(\theta_{n-1}\right)$ using formulae $(202)$.

\subsection{Numerical Algorithm for Crack Propagation}

As was mentioned above, a stepwise method to model crack propagation is used. At each propagation step, the crack is extended from the previous tip by a length increment $s$ in some direction (Figure 2) found from the propagation criteria $K_{1}=K_{1 C}, K_{2}=0$. At each propagation step the numerical algorithm to model crack growth can be summarized as follows:

1. Numerical solution of the equation (191) to define the DDs along the initial crack;

2. Calculation of the SIFs $K_{1}$ and $K_{2}$ by using the formula (194);

3. Adjusting the uniform pressure on the boundary of a wellbore (and fracture for slow rate) to reach the condition $K_{1}=K_{1 C}$;

4. Definition of the deviation's angle $\theta$ in which $K_{2}=0$ (by rotating the increment); and

5. Smooth approximation of the new crack path by a circular arc (if $\theta \neq 0$ ) or a straight segment (if $\theta=0)$.

All these steps except for the third one has been described in details [102] for the general case of piece-wise homogeneous elastic plane (without taking into account the presence of a circular opening). Due to linearity of the problem, the pressure to propagate the fracture can be found from the solution of linear equation

$$
p=a K_{1 C}+b
$$

where $a$ and $b$ are some constants. These are found from the numerical solution of the equation (191) for two different values of $p$. These values are chosen using the propagation pressure calculated at the previous step. 


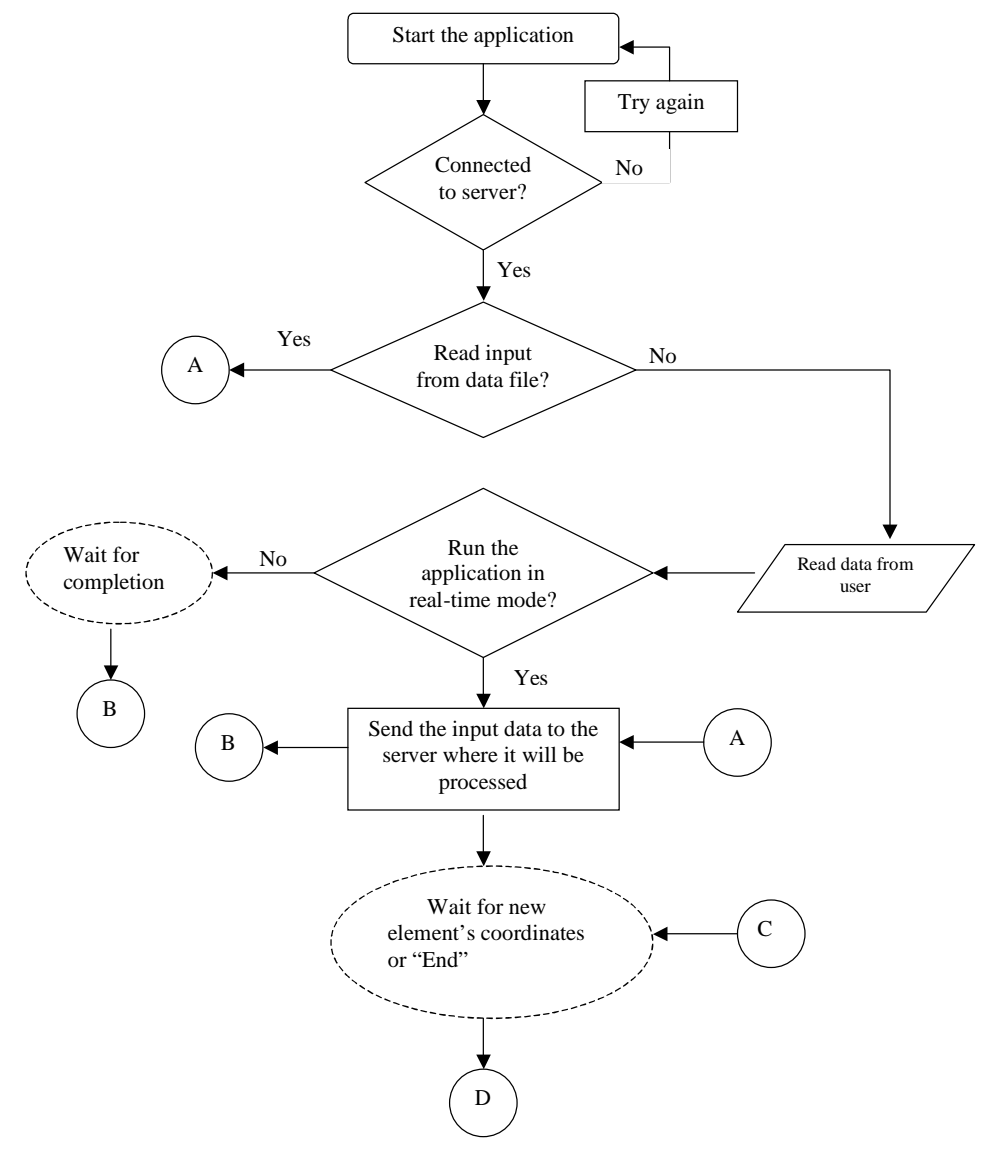

Figure 95: Part 1 of the fowchart.

\section{Appendix B}

\subsection{Graphics User Interface (GUI)}

The current architecture of the crack propagation application is depicted in Figures 95-98 below. The flowchart explains the connectivity of the GUI and the program that actually generates the output files necessary for plotting and viewing the outcome of numerical experiments. The dashed-oval depicting the "wait for completion" state is entered when the user sends the input data to the server and is exited when the client receives a message from the server indicating that the server has completed the execution. It is at this stage that the client can download the output files from the server. The flowchart does not depict the order of execution of the application but only depicts the steps a user will follow to view the results of the provided input data with many or no intermediate steps.

In order for a client to use the application, the client has to first get connected to the server. Currently, the server can serve only one client at a time. Thus, a connection can be established only if the server is idle. The information that is required to connect is shown in Figure 99 (the default settings connect to the server). Once the connection is established, the user may begin using the program (a READ.ME file accompanying 


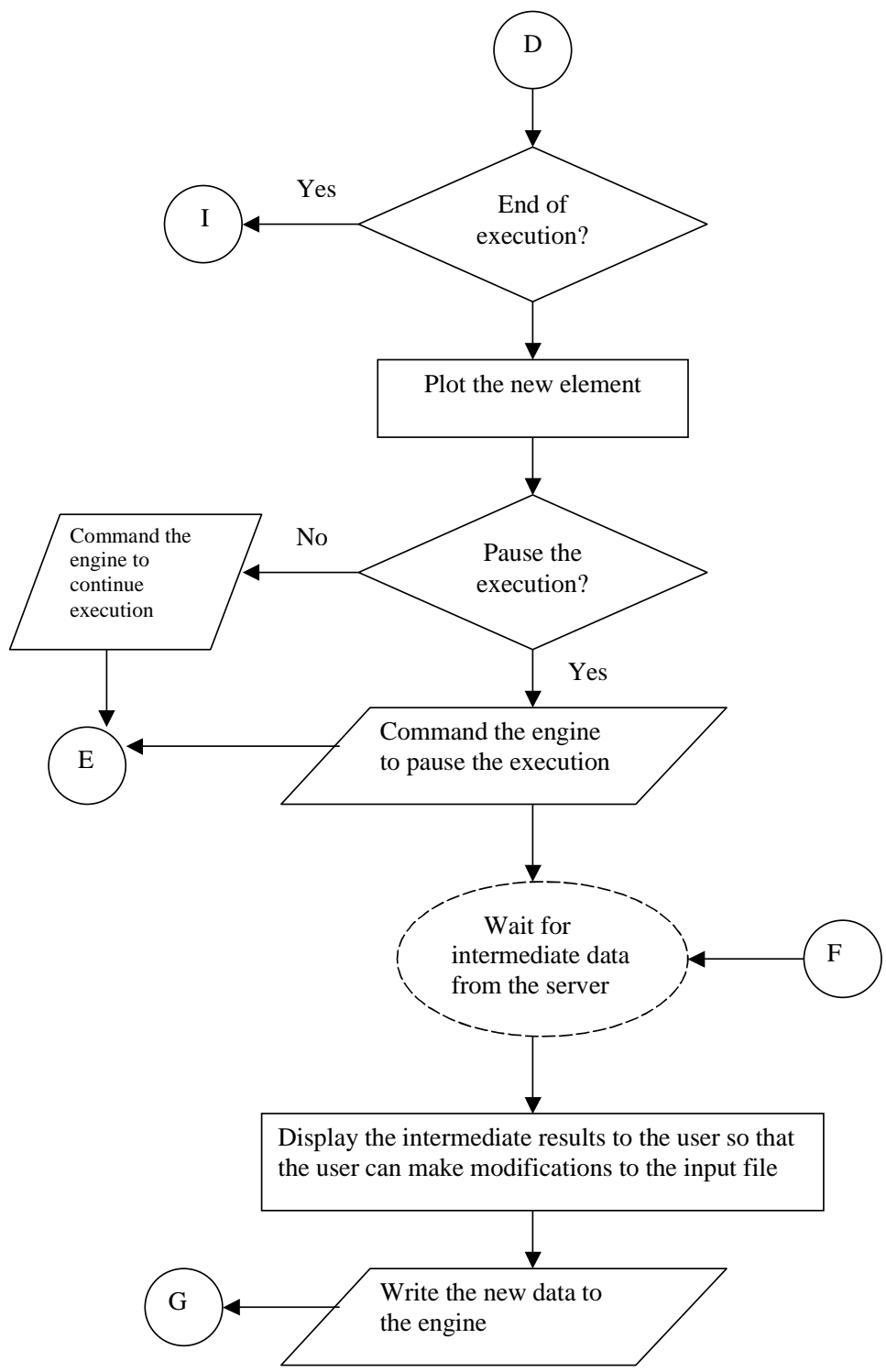

Figure 96: Part 2 of the flowchart. 


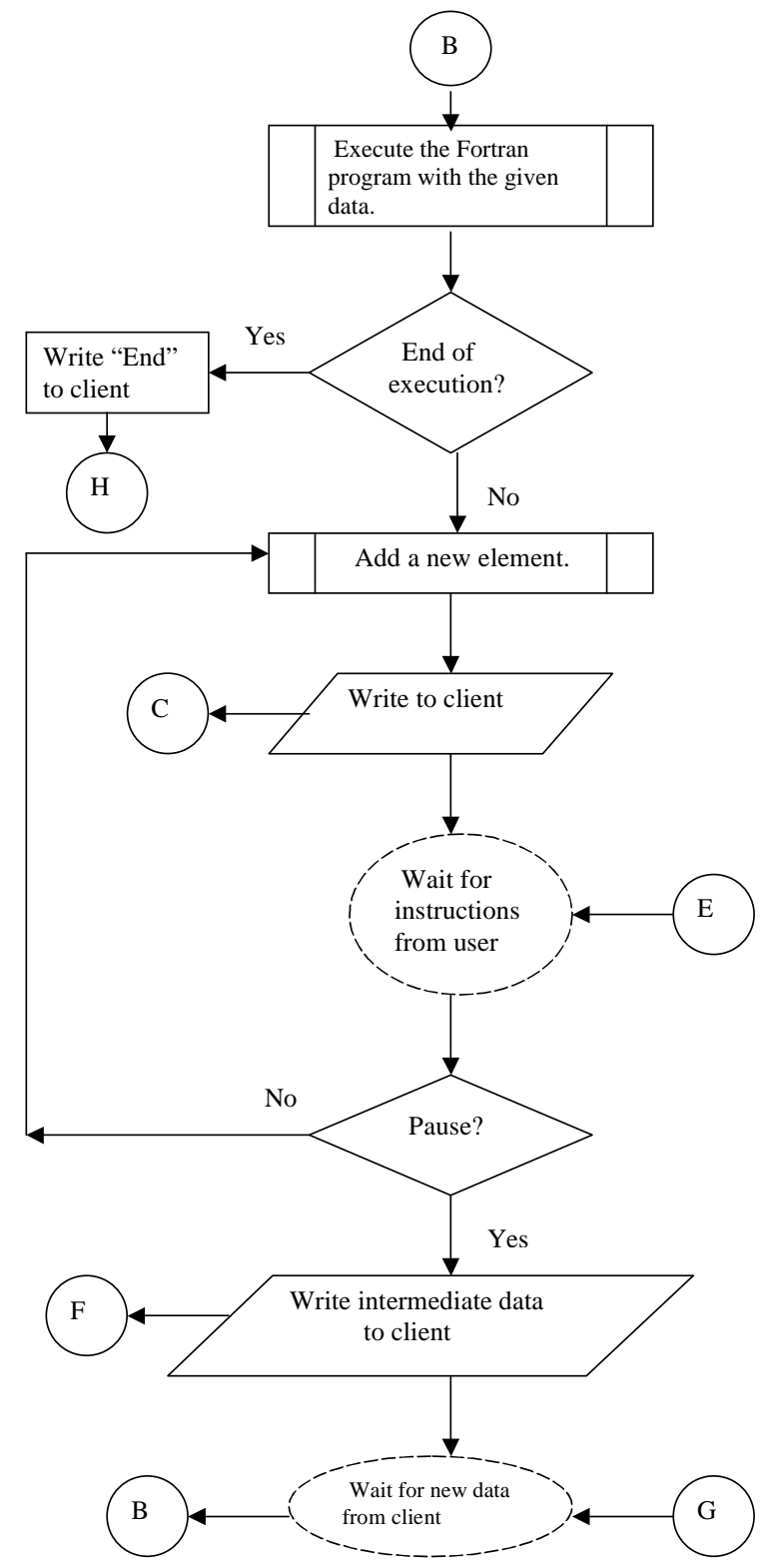

Figure 97: Part 3 of the flowchart. 


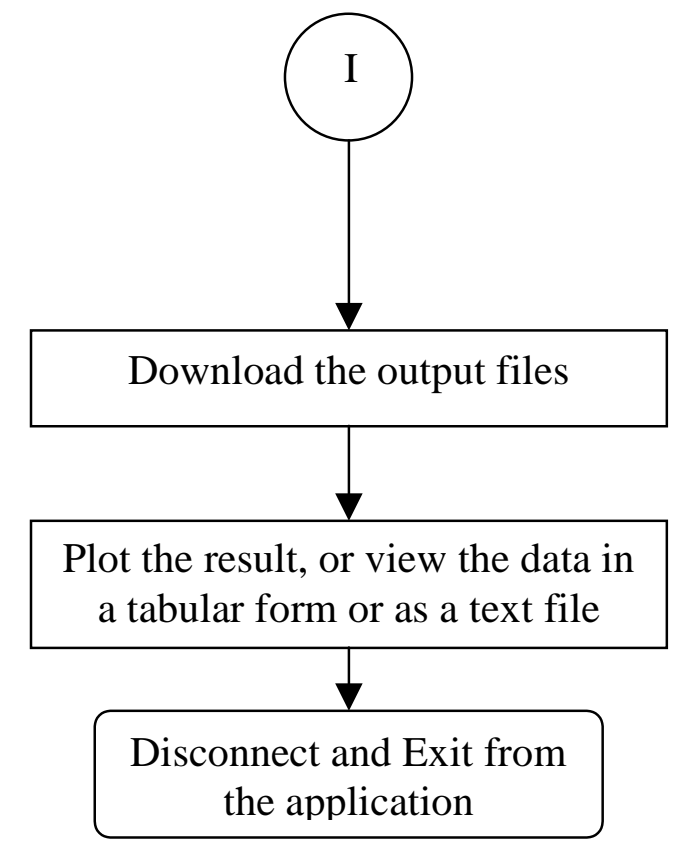

Figure 98: Part 4 of the flowchart.

the program provides a detailed account of the steps involved) using the input/output procedures described below.

\subsubsection{Input procedures:}

The input parameters can be entered via an input file or on the screen (real time mode). The client can use a submenu to choose the option of an existing input file (Figure 101) entering data in real-time mode.

- Input data:

This sub-menu allows the client to enter the various parameters of the problem on the screen. At the end of the second screen the client has the option to either click on the "Run" button to run the problem in

\begin{tabular}{|c|c|}
\hline \multicolumn{2}{|c|}{ Enter the network setup information $-[|\square| x$} \\
\hline Socket address & 134.129.143.109 \\
\hline Port number & 9970 \\
\hline Connect & Help \\
\hline
\end{tabular}

Figure 99: Window showing establishing a connection to the server. 


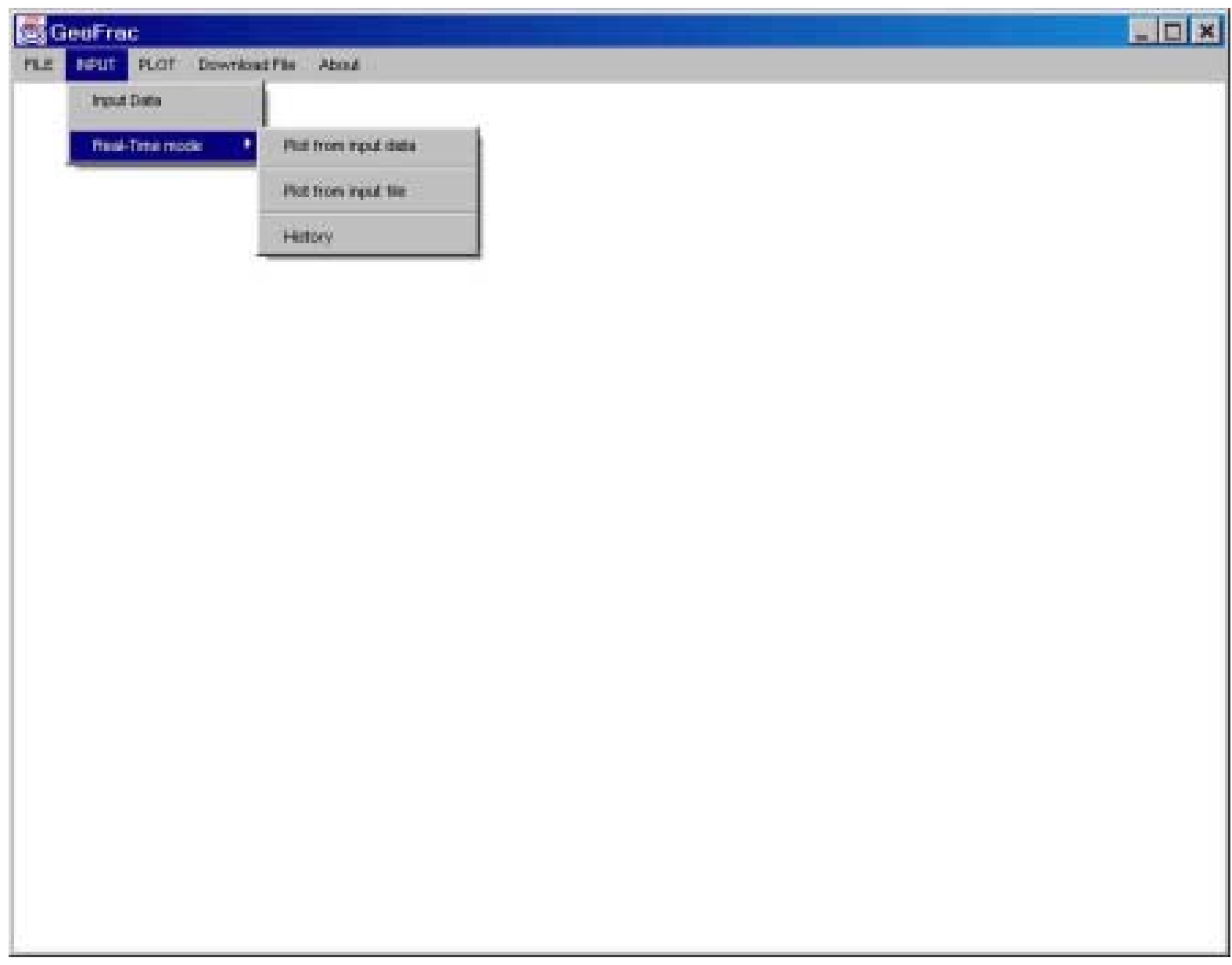

Figure 100: Window showing program access and run options. 


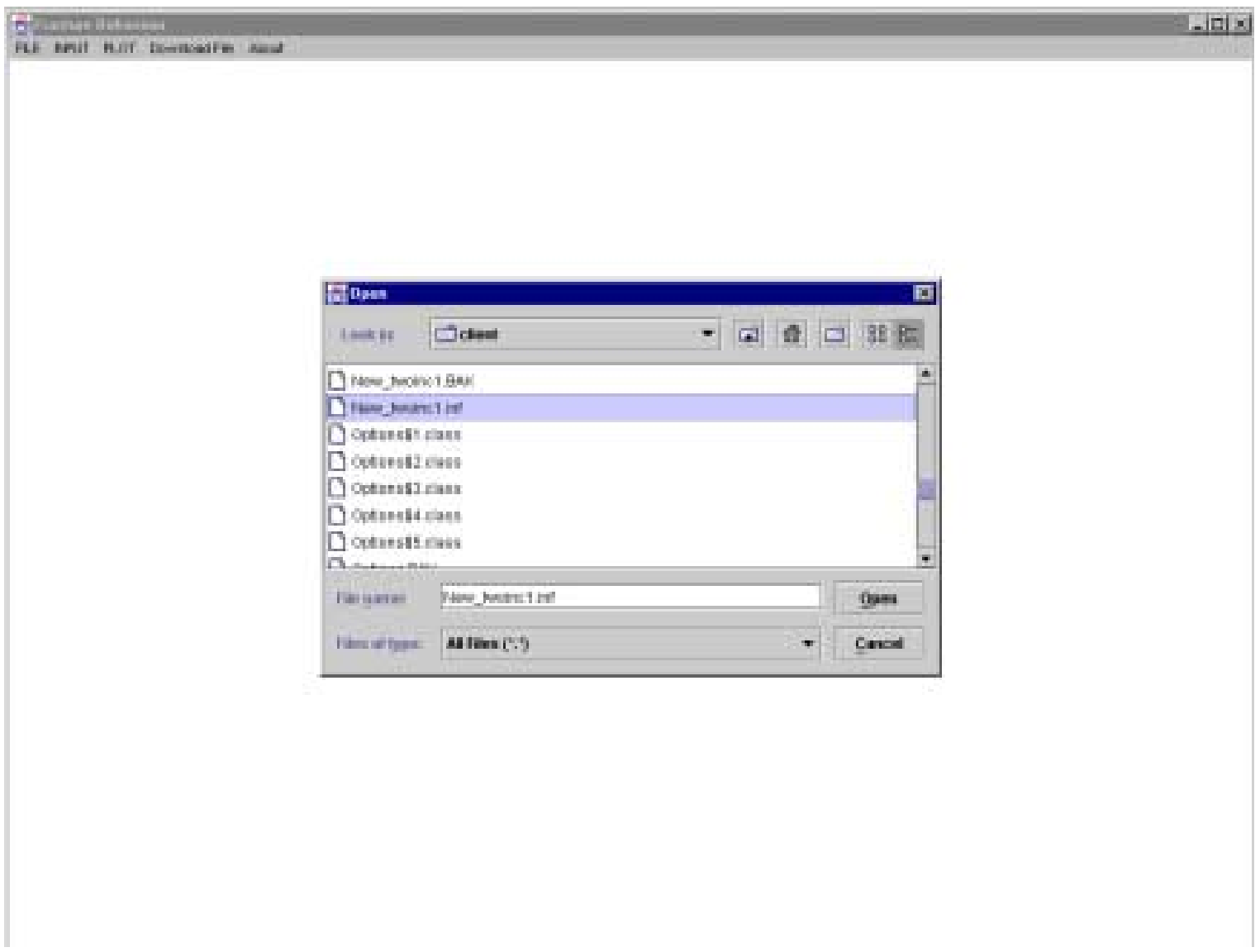

Figure 101: Window showing accessing a file. 


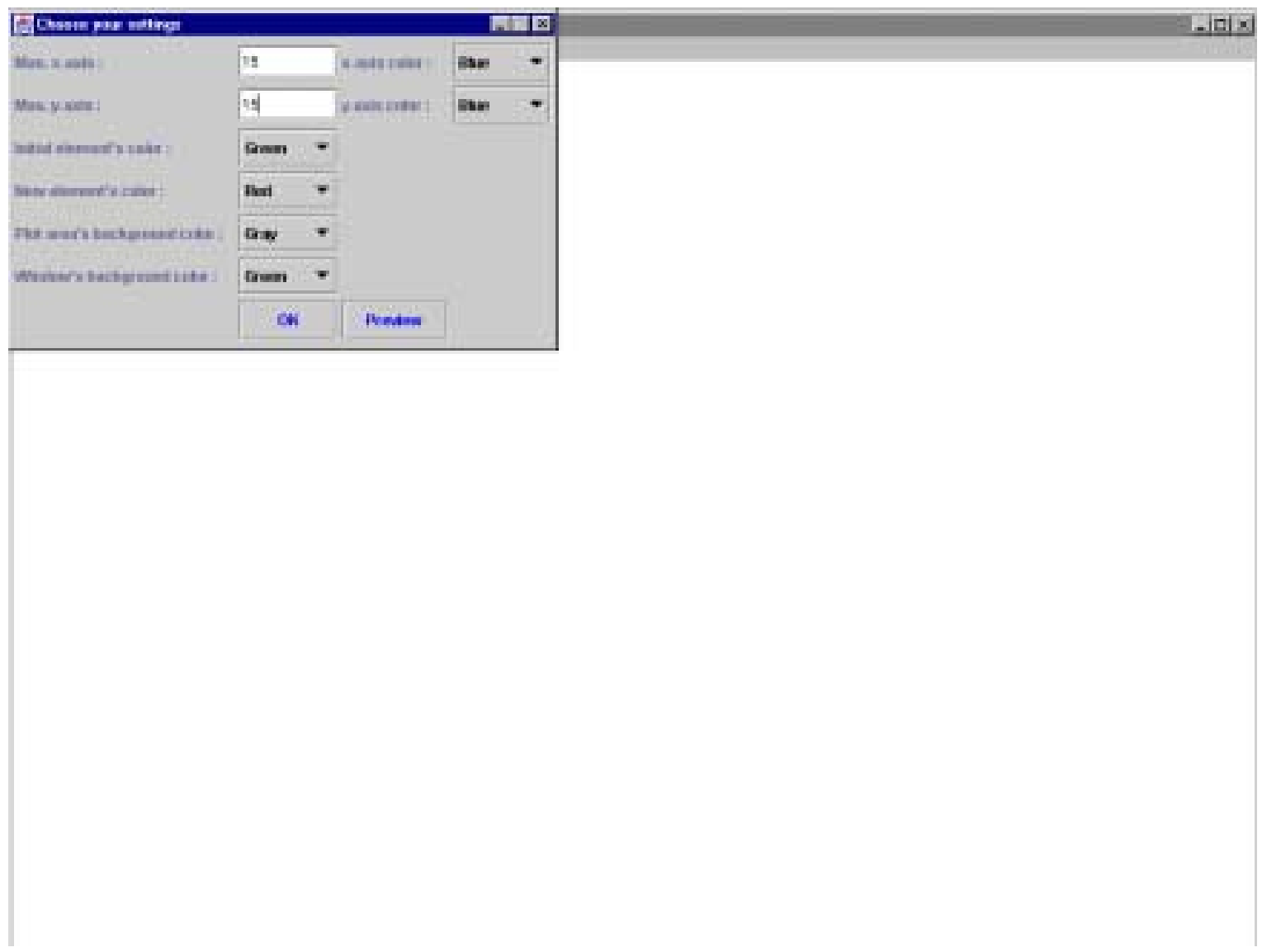

Figure 102: Window for choosing output plot color, size, etc. 
the non-real time mode, or click on "Cancel". Clicking on the "Cancel" button saves the input entered in a file named "input.inf".

(a) Real-Time mode:

After the user has completed the input on the screen, then the problem can be solved in real-time mode. There are two choices; the first one is to click on the "Plot from input data" under the Real-time mode sub-menue, and the other is to click on the "Plot from data file"

\section{(b) Input from Data File}

If the client does not wish to enter the input parameters via input screens but has the input data in a file, the client can make use of this sub-menu. However, the input file should have a specific format as explained below. An example input file is shown in Figure 103. When the client clicks on this, an "Open" screen pops up (Figure 101) that allows the client to choose the input file. After the client chooses the input file, a screen pops up (Figure 102) allowing the user to choose the initial element's color, new element's color, window's color, plot area's color, the axes color and the range for both axes. By setting appropriate values for the range, the client can have a better granularity of the plot (i.e., it produces a zooming effect).

If the client enters data using a data file, then the following format should be used:

Omega_iter, N_max_iter, eps_iter

N_segm, rigid_body, coef_sym, LK, LR, Kernel_type, N_new_elem, K1C, TR

Shear_mod_inf, pois_inf, stress_inf_xx, stress_inf_yy, stress_inf_xy, X0, Y0, sym_x, sym_y

N_as_l, N_as_r

Elem_as_l(i)

Elem_as_r(i)

Segm_beg_x, segm_beg_y, segm_end_x, segm_end_y, G1, pois_l, G, pois_r, segm_bc_type, X

XC, YC, segm_type, N_segm_elem, add_press, segm_bc_normal, segm_bc_shear

Where:

Omega_iter: Iteration Parameter

N_max_iter: Max number of iterations

Eps_iter: Precision parameter

N_segm: Number of global segments

Rigid_body: set to 1 for correction for rigid body motion (set to 0 when no need to correct)

Coef_sym: Specifies the line of semmetry, $=1$ if there is no sym-ry,

$$
\begin{aligned}
& =2 \text { if } \mathrm{x}=\mathrm{XSYM} \text { is the axis of the sym-ry, } \\
& =3 \text { if } \mathrm{y}=\mathrm{YSYM} \text { is the axis of the sym-ry, } \\
& =4 \text { if } \mathrm{x}=\mathrm{XSYM} \text { and } \mathrm{y}=\mathrm{YSYM} \text { are the axes of sym-ry }
\end{aligned}
$$

LK: number of divisions from element's beginning where the 1st collocation point is placed

LR: number of divisions. This means that if e.g., the first collocation point is placed at $1 / 6$ of the segment's length, the second collocation point would be located at $5 / 6$ of the length (measured from beginning of the element)

Kernel_type: 0 (1) if the plane without (with) a default hole

N_new_elem: Number of elements that needs to be added

$\mathrm{K}_{1 C}$ : rock fracture toughness 
TR: normal traction on boundary of opening

Shear_mod_inf: the shear module at infinity

Pois_inf: Poisson's ratio at infinity

Stress_inf_xx, stress_inf_yy, stress_inf_xy: the stresses at infinity

(X0, Y0): the complex number of a point for fixing the rigid body movement (any point but better to choose a good line).

Sym_x: axis of symmetry

Sym_y: axis of symmetry

N_as_l: number of elements with left asymptotic

N_as_r: number of elements with right asymptotic

Elem_as_l(i): element Numbers of elements with left asymptotic

Elem_as_r(i): element Numbers of elements with right asymptotic

Segm_beg_x, segm_beg_y: Segment's beginning

Segm_end_x, segm_end_y: Segment's end

G (G1): the shear mod. on the right (left) side of the element

Pois_r (pois_l): the Poisson's ratios on the right (left) side of the element

Segm_bc_type: Segment boundary conditions type:

$=1$ if tractions are given

$=2$ if $\mathrm{DD}$ are given

$=3$ if the relation segm_bc_normal + isegm_bc_shear $=C A *(U N+i U S)$ is given

$\mathrm{X}: \mathrm{CA}$

(XC,YC): the center of the circ. arc; enter $(0 ., 0$.$) for the straight el-t,$

Segm_type: equals 1 for straight element, equals 2 for circular arc

N_segm_elem: Number of equally spaced elements for a segment

Add_press: Paramter indicating if uniform pressure will be applied to a boundary:

0 if the pressure will not be adjusted

1 if the pressure will be adjusted

Segm_bc_normal: Normal boundary conditions at a segment

Segm_bc_shear: Shear boundary conditions at a segment

When the input data contains complex coordinates, a comma should not be used to separated them. The input file should NOT contain comments. 


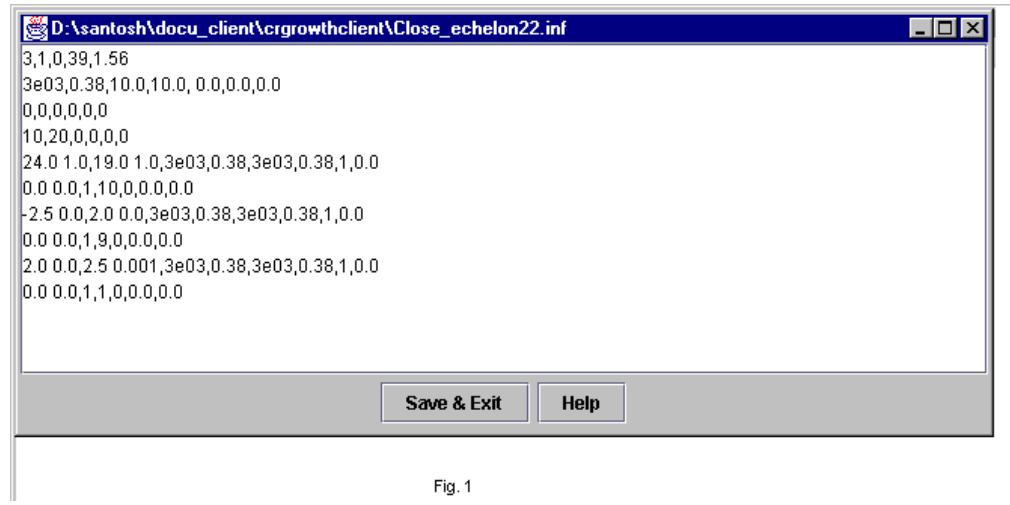

Figure 103: Window showing an input data file.

\subsubsection{File handling procedure:}

OPEN : As shown below, by using "Open" the input file or output file can be viewed. The screen that is displayed when the user chooses to open a file is shown above. The chosen file is opened in an editor as shown in Figures 104 and 105.

Opening a file using the "Open" submenu under the "File" menu, allows the user to make modifications to the file and the changes will be recorded when the editor is closed. If the user wants to run a simulation using the modified file, typically the following steps are used:

- Click on the "Input" menu

- Click on the "Real-Time mode" menu

- Click on the "Plot from input file" menu

- Traverse through the entire tree, locate the file and then run the problem

This last step usually becomes tedious when the user wants to run the same file more than once (with some modifications). The new version of the application brings some relief to the user in this regard. When the user opens a data file and makes changes to it in the built-in editor as described above or runs any data file, the "path" to the file is saved under the "History" submenu (Figure 106). Thus, the user need not have to traverse the directory tree to reach the same file, but instead has to just click on the "History" submenu under the "Real-Time mode" submenu to display all the files used in the current session (Figure 106).

- About:

This gives a general description of the program that the GUI executes. To close the screen, click anywhere within the gray part of screen (Figure 107). 


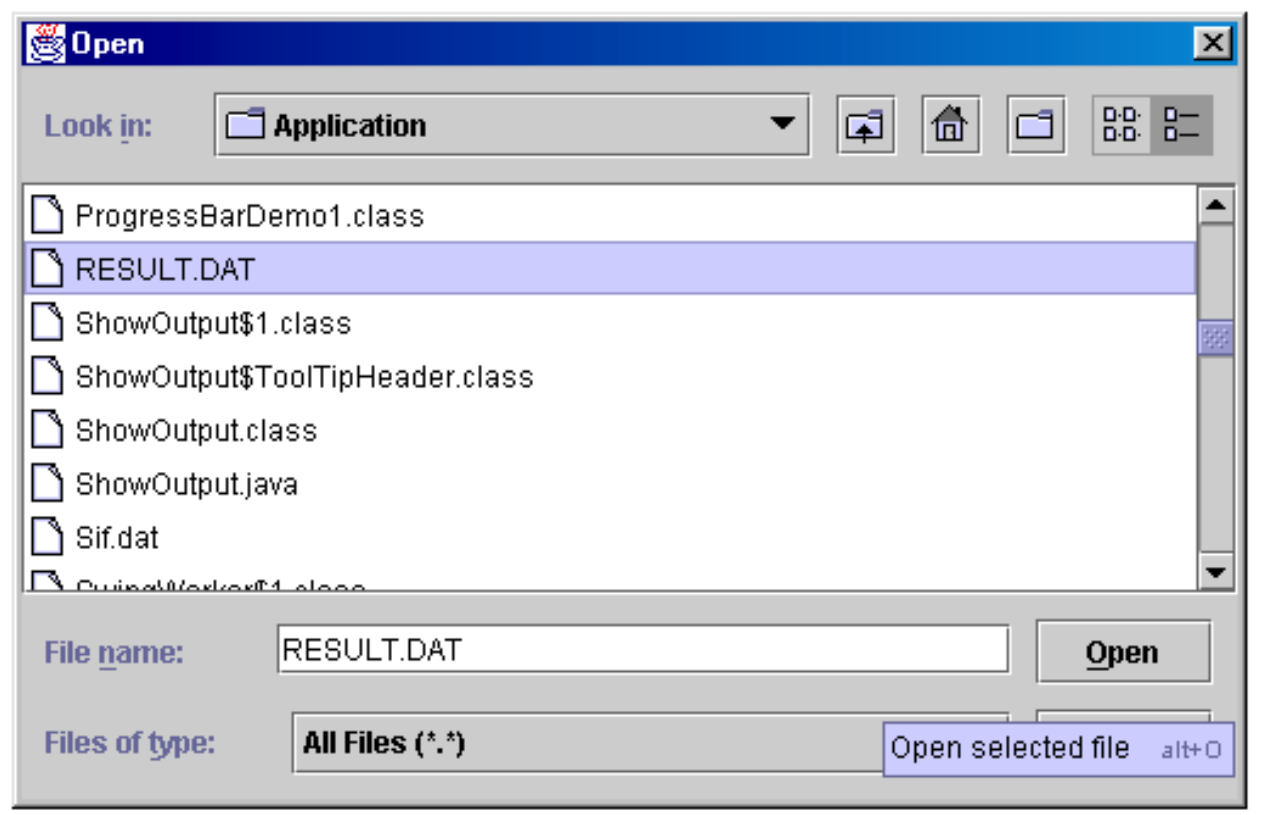

Figure 104: Window showing file access procedure.

Details of Real Time Input Procedure: This is accomplished by using two screens (tool tips are provided for a number of the parameters). The first screen is shown in Figure 108. The user cannot go to the second screen without entering all the input values of the first screen; the same holds for the second screen. After all the parameters are entered on the first screen, the user can proceed to the next input screen by clicking on the "Proceed to the next page" (Figure 108). The screen iterates for the total number of fracture segments entered by the user. The current segment is displayed on the bottom of the second screen. The user can enter the values for the successive segment by clicking on the "Next Segment" button (Figure 109). After all the input parameters have been entered, the "Next Segment" button is disabled and the "Run" button is enabled (Figure 110). The user can run the program by clicking on the "Run" button. A message pops up telling the user that he would be notified when the server has finished executing the program. At the same, a progress bar appears indicating that the server is currently running the program. After the server completes the execution, it sends back a message notifying the client that the server has completed executing the problem and that the user can download the output files (Figure 111).

\subsubsection{Simulation output}

- Downloading output files:

Filename: After the user is finished with entering all the required parameters and after the server has notified the client that the server has finished executing the program, the user can download the output files. To determine which files need to be downloaded from the server, click the "HELP" button in the download screen. 


\begin{tabular}{|c|c|}
\hline geroblem Description & $-\nabla x$ \\
\hline the case of plane & 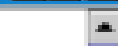 \\
\hline NUMBER OF BOUNDMR'Y ELEMENTS $=8$ & \\
\hline ELEMENTS WITH RIOHT ASMMPTOTIC & \\
\hline 8 & \\
\hline ELEMENTS WITH LEFT ASMMPTOTIC & \\
\hline X\%-COMPONENT OF FIELD STRESS $=0.0000 E+00$ & \\
\hline Y-CONPONENT OF FIELD STRESS $=0.1000 E+01$ & \\
\hline XY-COMPONENT OF FIELD STRESS $=0.0000 E+00$ & 2 \\
\hline SHEAR MODULUS AT INFINITY $=0.5000 E+00$ & \\
\hline POISSONS RATIO AT INFINTY $=0,0000 E+00$ & - \\
\hline
\end{tabular}

Figure 105: An example of an output file generated by the program (Result.dat).

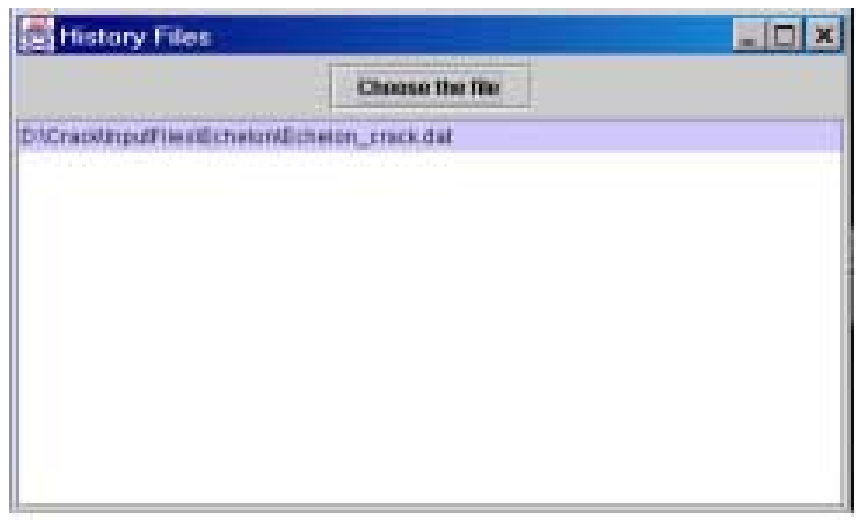

Figure 106: Window showing the history of files accessed. 


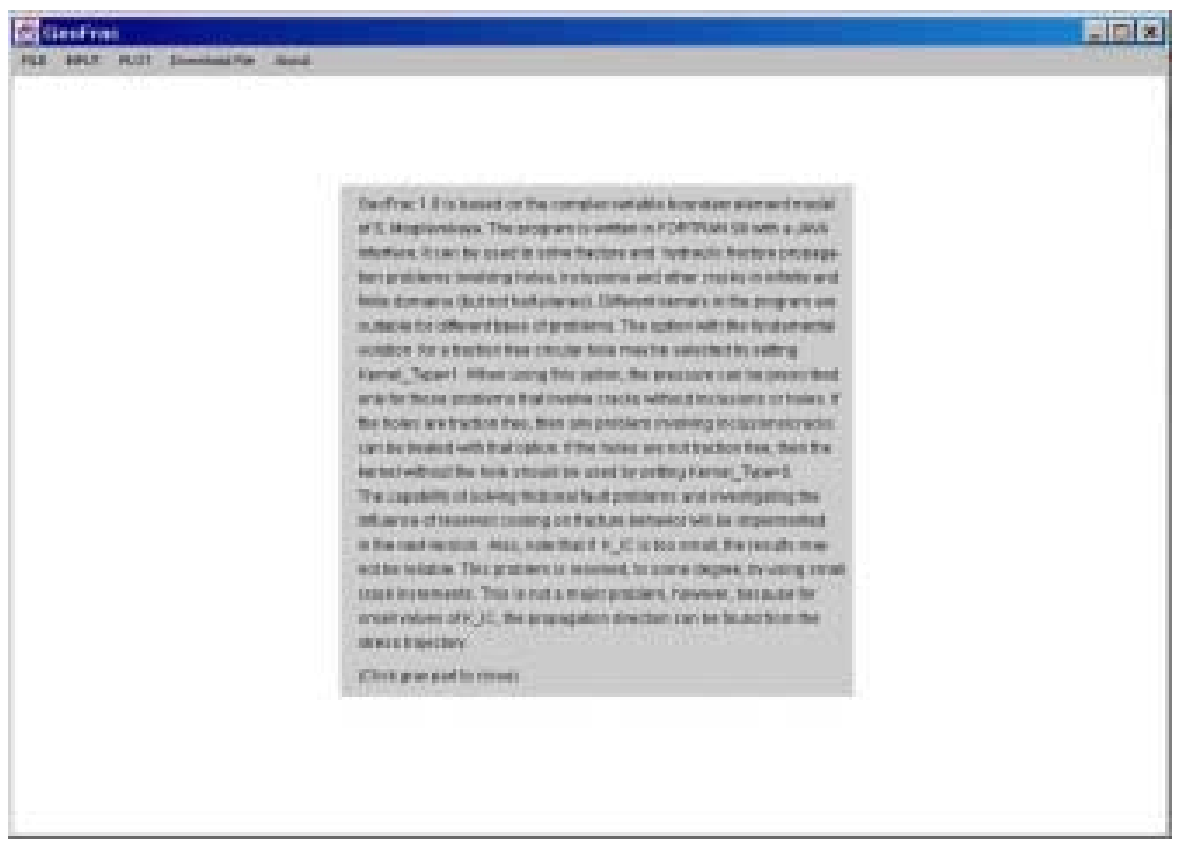

Figure 107: Window showing a brief description of the program and various engine options.

NOTE: A warning message appears when an attempt is made to download a file that is not present on the server's disk.

- Viewing simulation results:

- Plot Result: This plots the results from the downloaded file "Result.dat". An example plot for a kinked crack near a borehole shown below (Figure 112):

Other files containing information regarding the problem geometry, boundary conditions, and other data of interest may also be viewed:

General Information: This describes the problem description, etc from the downloaded file "Output.dat". An editor similar to the one shown under "FILE -> OPEN" is opened.

- - Boundary Conditions: This represents the data in a tabular form from the downloaded file "Output.dat".

- Mesh Data: This represents the data in a tabular form from the downloaded file "Output.dat" (see Figure 113).

- SIF/Load Data: This represents the load and stress intensity factor (SIF) data in a tabular form from the downloaded file "Sif.dat" (see Figure 113).

NOTE: An attempt to plot or open any items without downloading the files will cause a warning message and a blank table is displayed. 


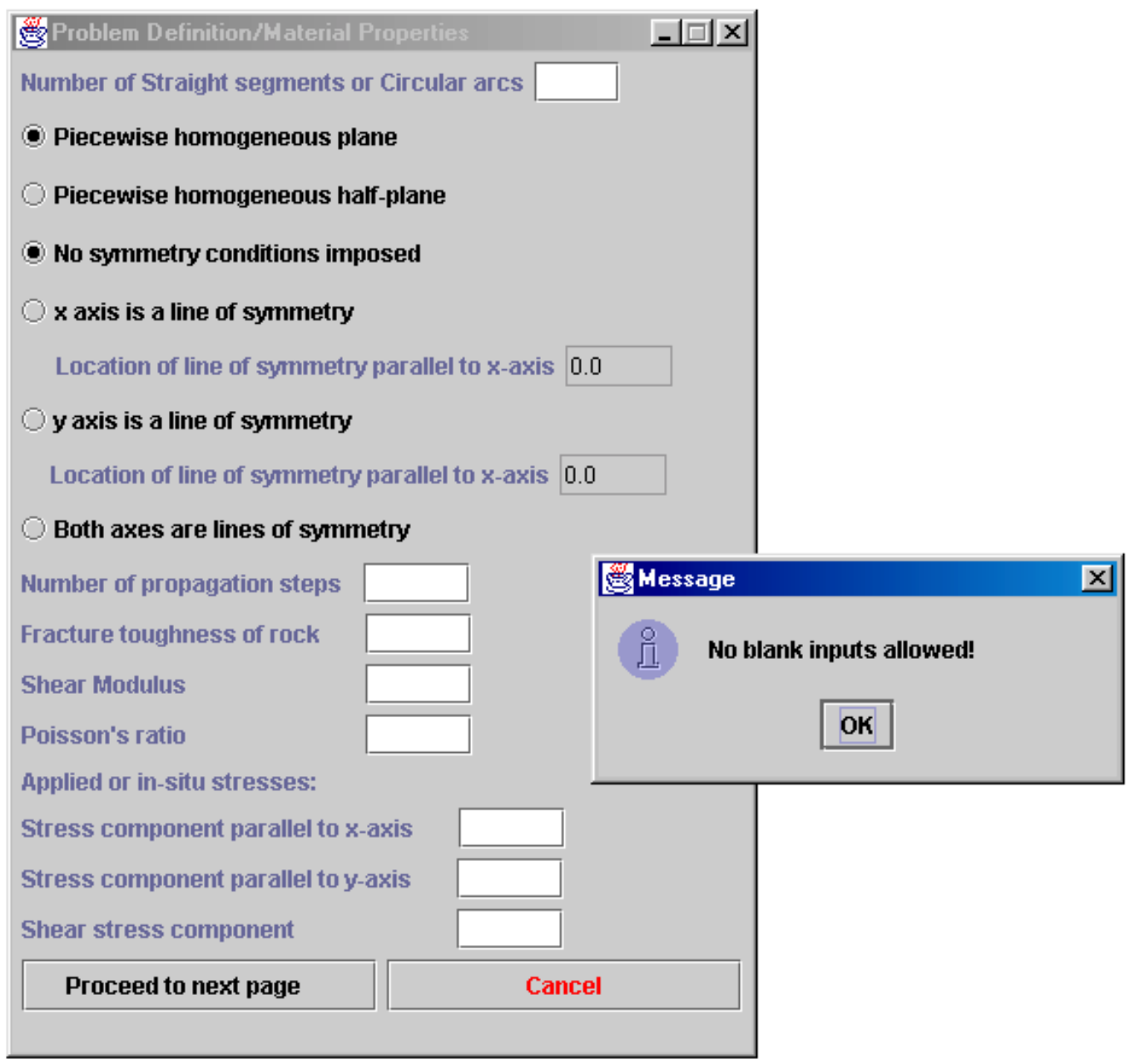

Figure 108: Window showing program's response to blank input lines. 


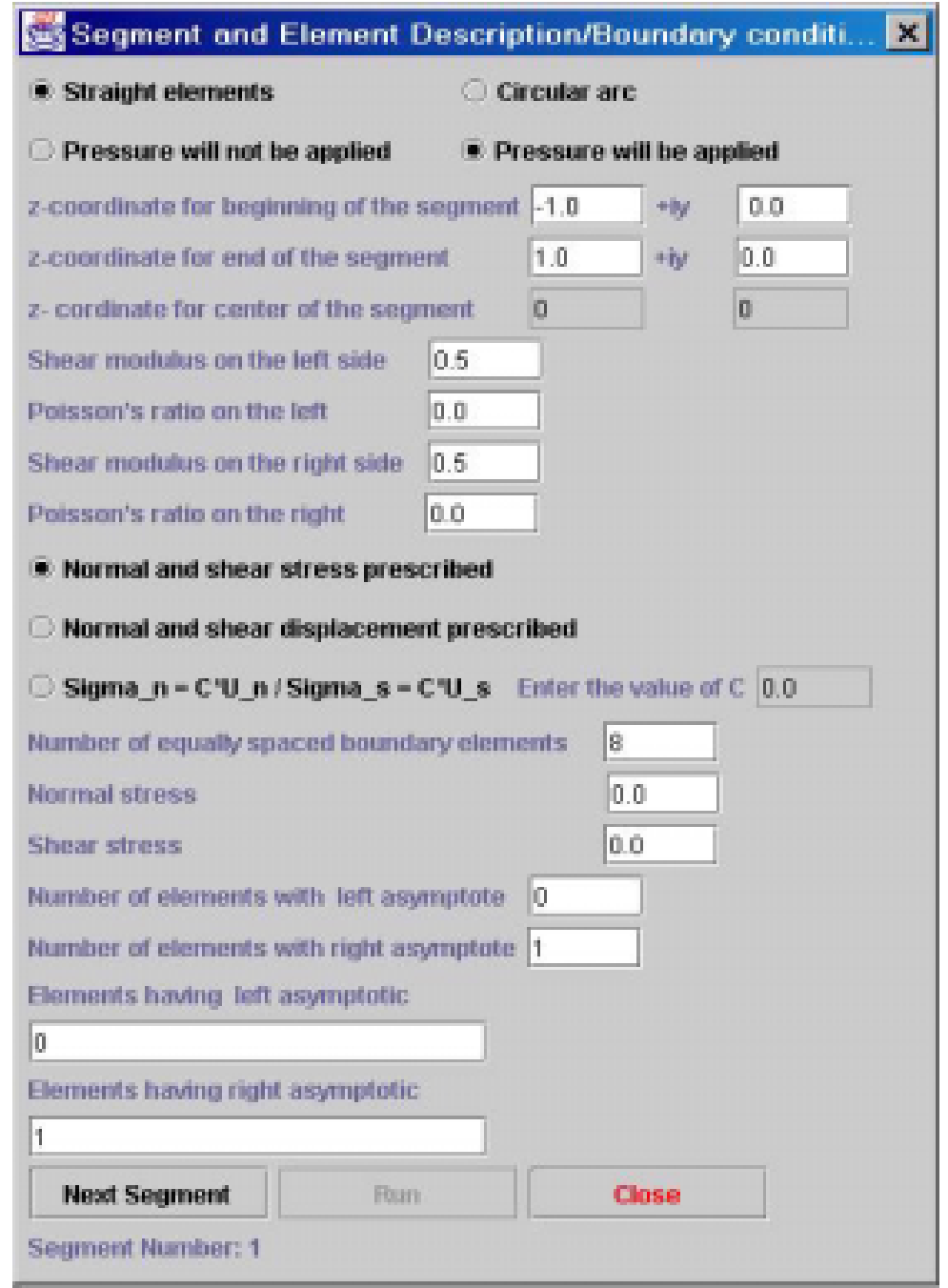

Figure 109: Window showing real time input. 


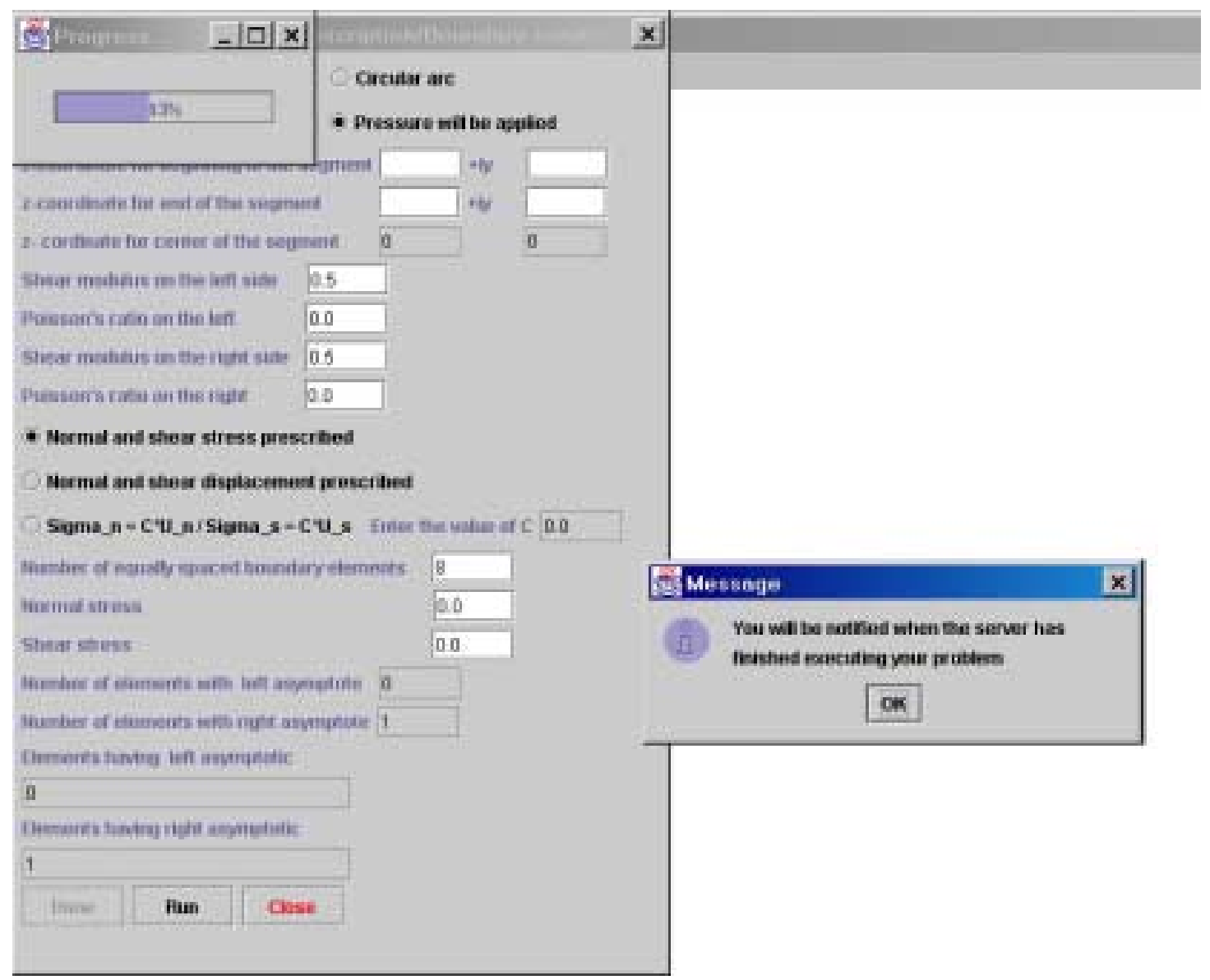

Figure 110: Window showing a message in response to a RUN command.

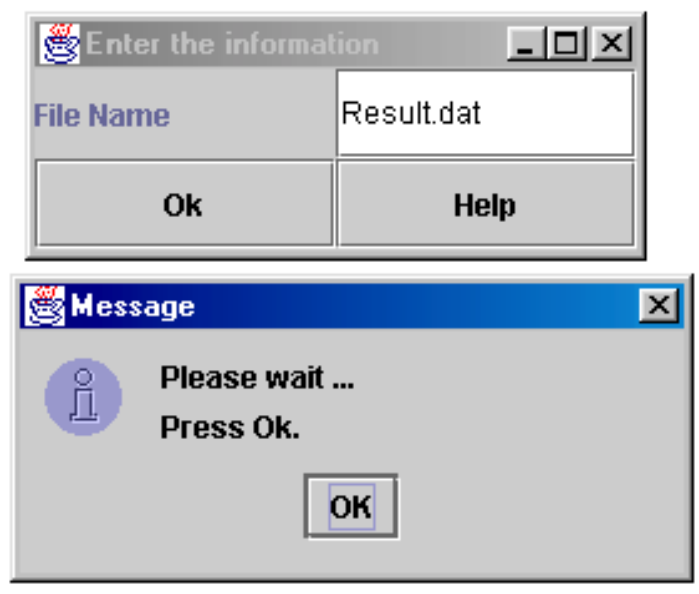

Figure 111: Window showing program's response to a download request. 


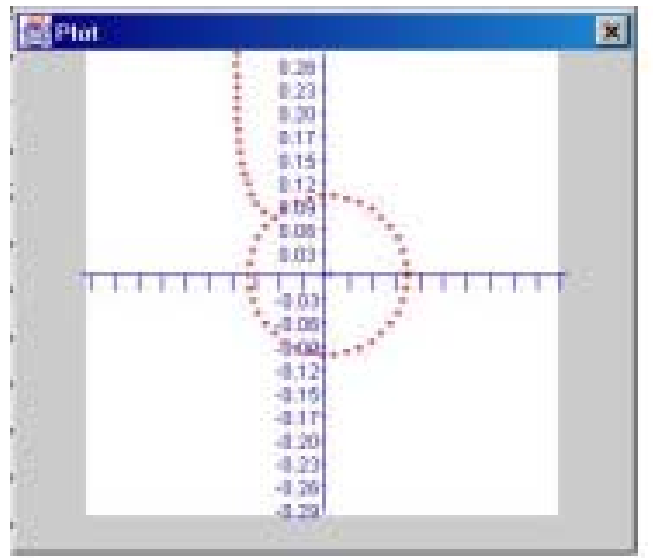

Figure 112: A plot generated from an output file.

\subsubsection{Real time output}

The application allows the user to communicate with the Fortran engine during its execution, through the interface. A feature has been added to the real-time plot of fracture propagation. It is a message that displays the current tip position. Therefore, every time a new element is added to the existing set of elements, the position of the tip is indicated to the user (Figure 114). The user is allowed to pause the execution of the Fortran engine to:

- View the propagation path

- View the various parameters related to new elements'

- Change the problem definition, element properties (element type, boundary conditions, etc) and command the Fortran engine to continue the propagation with the new modified parameters.

This feature can be activated while the window that is responsible for plotting the propagation path is active (a window is said to be active when the input focus is on it e.g., Figure 114). To pause the execution of the engine, the user can "press" any key. Doing so will cause a message to scroll at the top of the current window acknowledging the request (Figure 114). A window allowing the user to view the intermediate data and/or to run the modified data will then be displayed after a brief delay (Figure 116). This delay is due to the fact that the engine checks to see if the user has requested a pause in the execution only after adding a new element. However, if the user requests such an action during the execution of the routines involved in adding the new element, the engine continues to write out the coordinates of the new elements prior to pausing. Thus, between the time the user requests the engine to pause and the appearance of the 


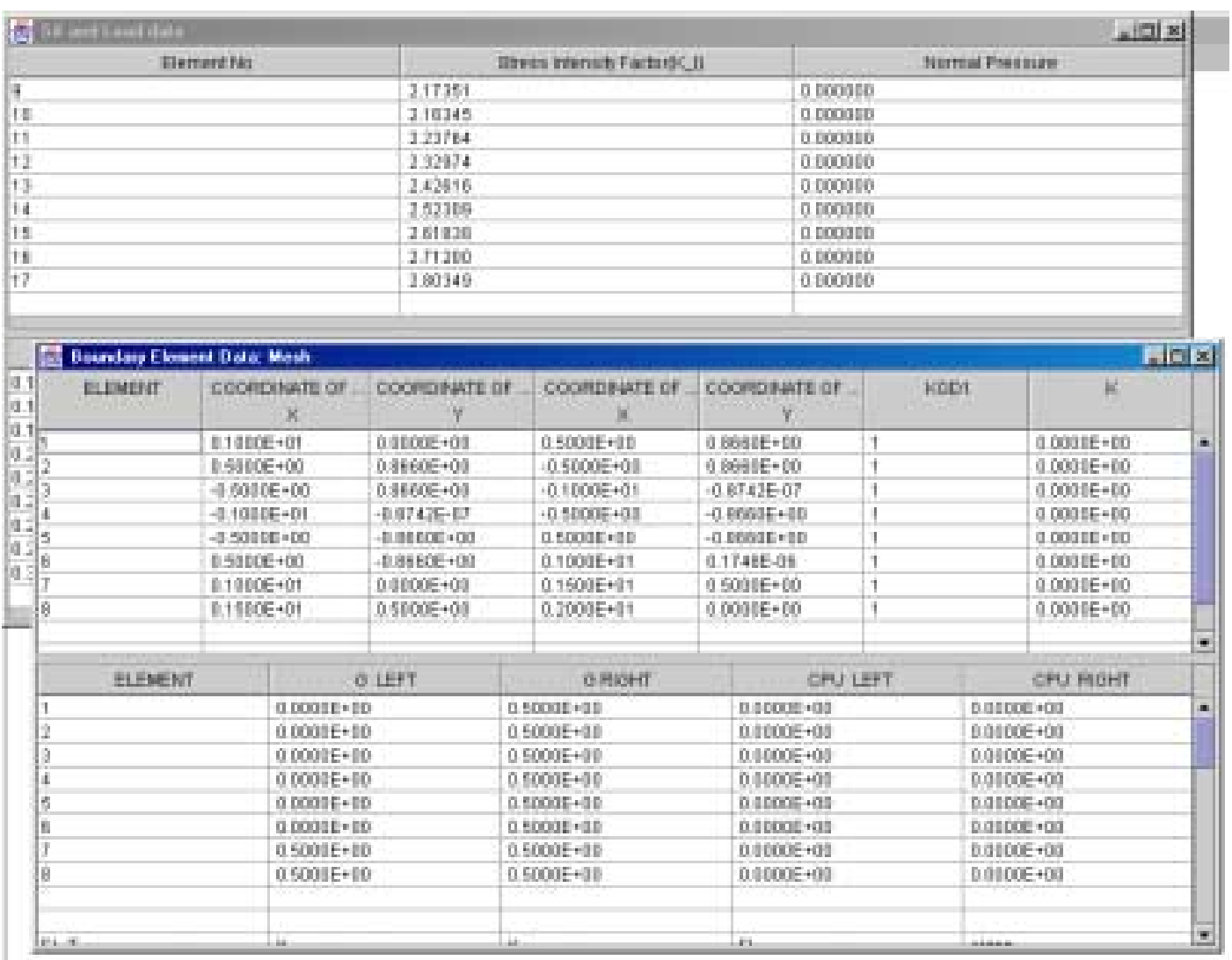

Figure 113: Window showing example output data files. 


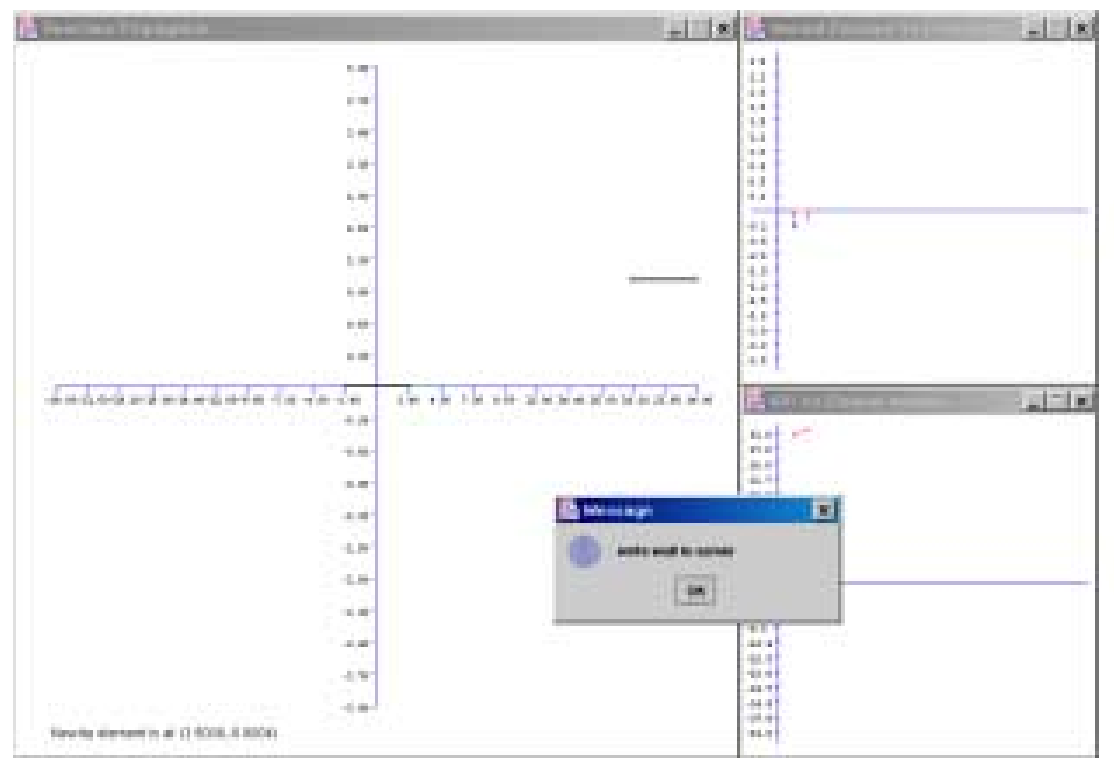

Figure 114: Window showing program's response to a pause command issued by user.

window, the coordinates of the intermediate elements are written out by the engine and are plotted on the screen. When the user chooses to view the intermediate data, a request is sent to the server to transmit the intermediate data. Recognizing this, the server sends out the data and this data is presented to the user. The user can then make modifications to the data presented (Figure 116). Clicking on the "Proceed to next page" button presents more information about each element (Figure 117). Clicking on the "Next" button displays information about the next element. Clicking on the "Cancel" button on either of the two windows cancels all the changes made to the data on that screen. Clicking on the "Continue processing with the changed data" button on either of the two windows closes the window after creating a file which includes the modified data as well as the portions of the intermediate data that were not modified. The user can continue the simulation by running the new input file. An example of this is shown in Figures 118 and 119 for the interaction of two echelon cracks. It can be seen that changing the shear stress boundary condition after the crack has propagated a few steps (Figure 114) causes the crack path to change, turning upward.

There are certain points the user has to remember when dealing with the current version of the application. Some GUI components are directly connected to the server. This means that events generated by the GUI are given as input to the server which acts accordingly. The client has to remember the following four points:

1) DO not use Ctrl-C to exit from the application. USE File->Exit instead. The server can handle only one client at a time. Therefore, when a client is connected to the server, other clients wishing to get connected are rejected. New clients are allowed into the system when a client exists. The only way the server can know if a client has exited is when the current client uses File-> Exit to exit from the application.

2) Allow the execution of downloading command to be completed. When a client clicks on "Download 


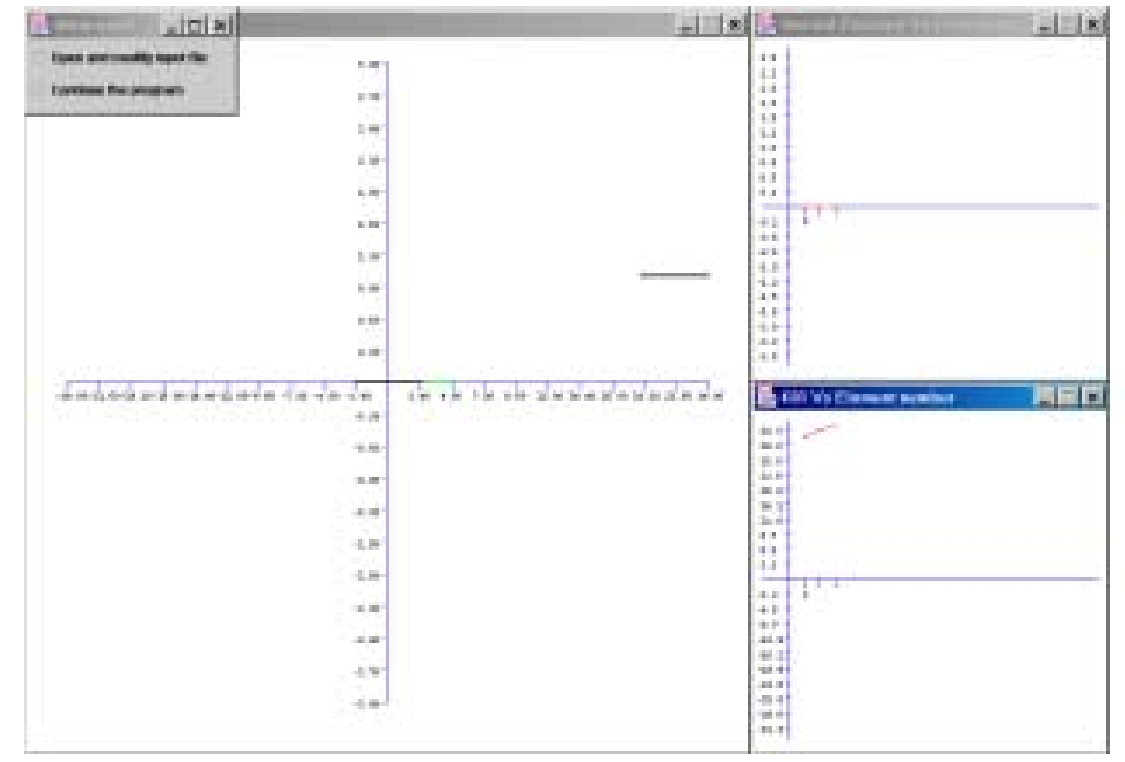

Figure 115: Window showing options upon pausing the program.

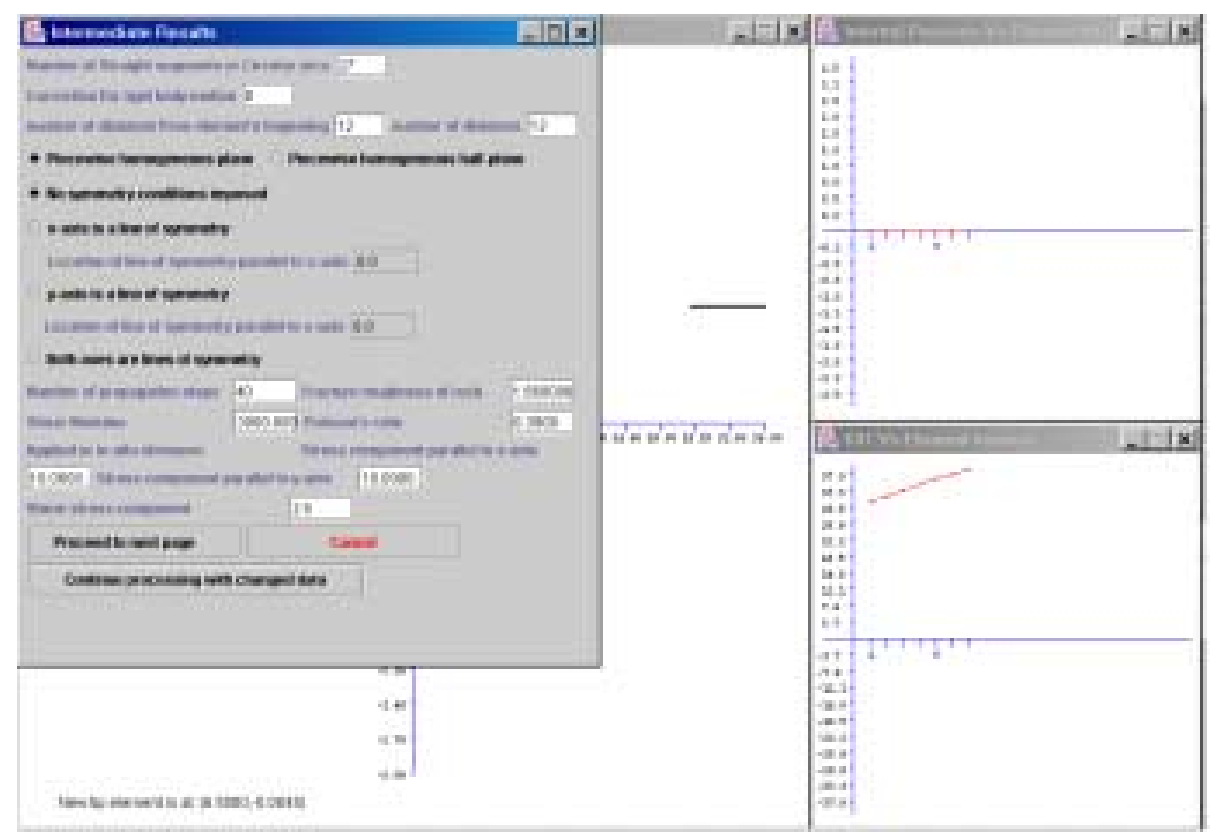

Figure 116: First window for changing input parameters after pausing the program. 


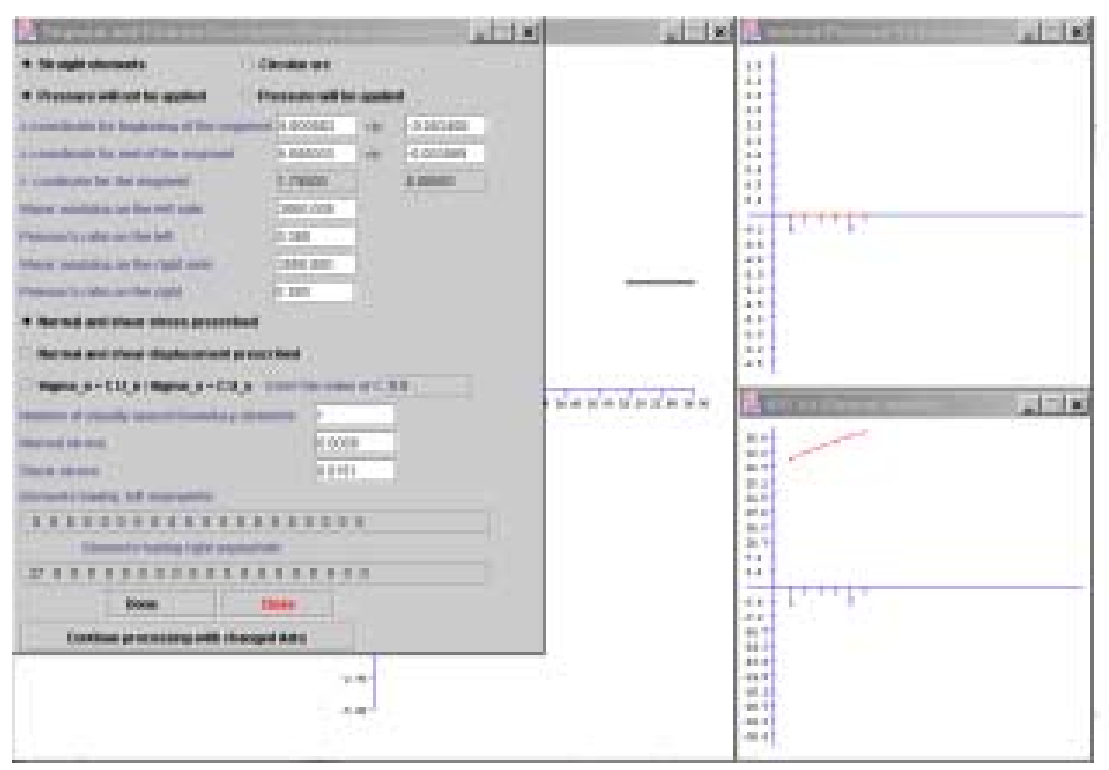

Figure 117: Second window for changing problem input parameters.

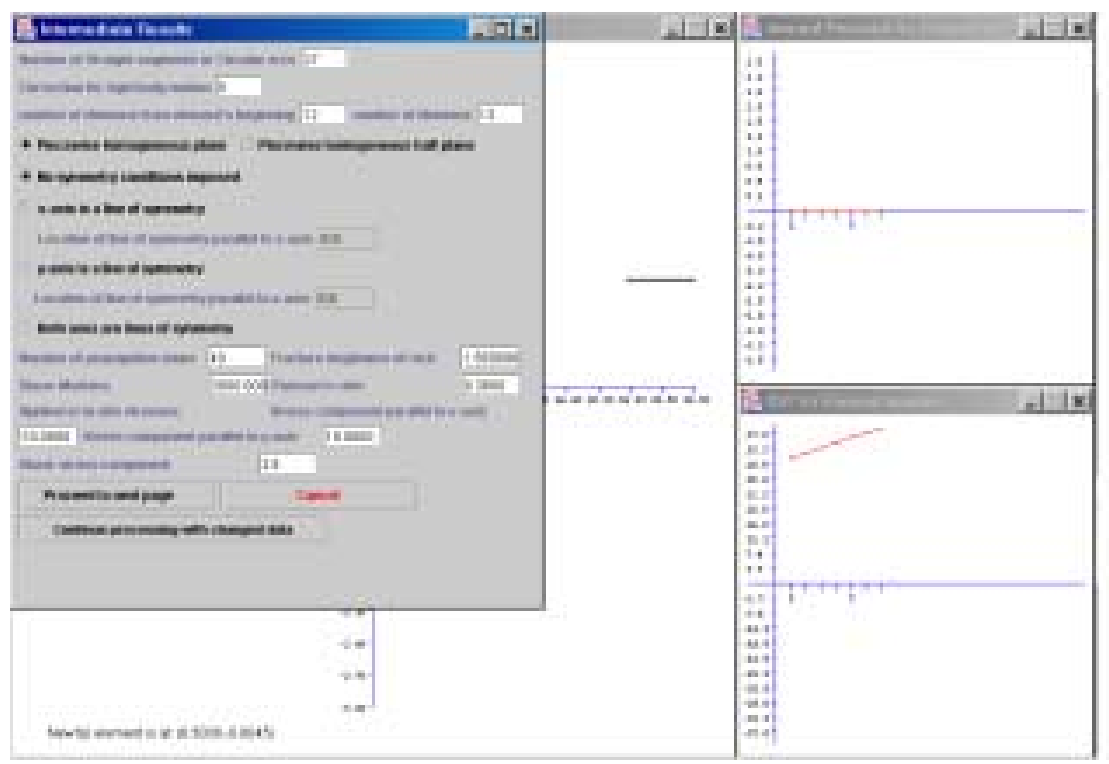

Figure 118: Third window for changing input parameters (e.g., shear stress) 


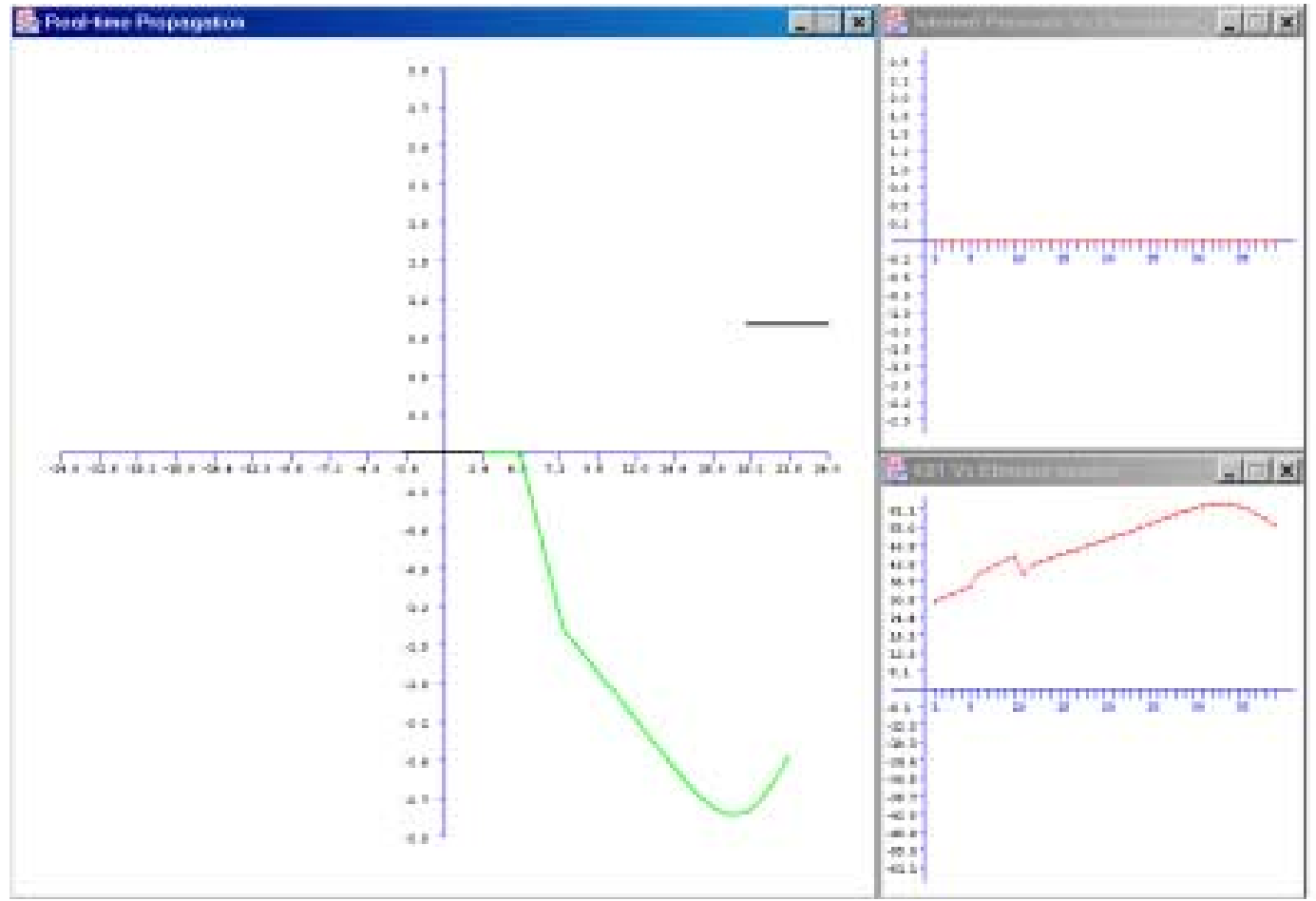

Figure 119: Real time illustration of the crack path adjusting to new boundary conditions. 
File", a command is issued to the server. Upon receiving this command, the server enters a state where it expects a file name to be sent by the client. If the client attempts to run the application at this time (or performs any other operation that involves the server), the server MIGHT hang. The client will have to force-quit the application and server will have to be restarted.

Examples Two examples are presented next. The first one is an example run using the file "Twoinc.inf". This problem illustrates the influence of circular inclusions and their elastic properties on fracture propagation. The upper circular inclusion has an elastic modulus five times larger than the lower one. The rock matrix's elastic modulus is one half the modulus of the lower inclusion. The horizontal and vertical in-situ stresses are 10 and 12.5 MPa, respectively. Briefly, the stiffer inclusion carries a higher stress than the softer one; it tends to rotate the stress field and attracts the fracture. The results are shown in Figure 66. The fracture is shown in green.

Figure 64 is an example of the interaction between two echelon fractures in an isotropic tensile in-situ stress field. The vertical distance between the two fractures is $1 \mathrm{~cm}$. The lower fracture is pressurized and allowed to propagate from the right. It follows a straight path initially, turning slightly away from the top fracture. However, the top fracture's distortion of the stress field attracts the propagating fracture and causes it to propagate towards it (in the direction of maximum stress). The fracture toughness in both examples is 1.6 MPa.m ${ }^{1 / 2}$. The sequence of program execution is listed below:

- Step 1: Choose "Plot from data file" located under the Real-Time mode sub-menu under Input menu. Open "New_twoinc1.inf", the formatted version of twoinc1.inf

- Step 2: When the client chooses the file, an "Options" screens pops up allowing customizing of the plotting window

- Step 3: In the next step, the client will be able to see the plotting window with the colors chosen. The plot (Figure 66) shows the actual propagation of the fracture. The red curve indicates the new elements that are added to the initial element. The black line segments indicate the initial elements.

- Step 4: After the program completes, the GUI indicates to the user that the program has completed its execution. Now, the client can download all the output files generated by the program.

\subsubsection{Current limitations}

The program limitations pertain to the engine and the interface. The engine or the mathematical models implemented need to be improved to handle fracture intersection, non-linear frictional sliding on cracks and joints, as well as to allow mixed mode fracture propagation. Also, fluid mechanics analysis should be implemented to rigorously couple fluid mechanics to solid deformation as well as thermoelastic effects.

The program currently allows for 20 tip elements and this needs to be increased later. This is a minor matter and can be addressed by modification of array sizes. Dynamic array allocation would speed up the program. The symmetry feature may not work properly in this version of the code and its use is not recommended.

Currently, the capability of modeling fracture propagation while considering thermoelastic stresses associated with fluid circulation is not available through the user interface. The improvement of the interface for 
this purpose is not a major matter and will be completed in the next version along with other improvements such as the nonhomogeneous thermoelastic CVBEM model.

Currently, the server is set up to handle only one user at a time. However, this issue can be addressed readily. Other limitations include the inability to plot or contour the stress distributions. Moreover, the mesh generation scheme is somewhat rigid. It is anticipated that using "mouse events" for input generation will remedy many of the existing shortcomings. 


\section{Appendix $\mathrm{C}$}

\subsection{Poro-thermoelastic Boundary Element Method}

The fictitious stress (FS) and the displacement discontinuity (DD) techniques are indirect boundary element methods. The former is based on the fundamental solutions to the problems of fluid/heat source and point force in an infinite solid; the latter utilizes similar singular solutions with a point DD used instead of a point force. These indirect methods have proven versatile for solving geomechanics problems involving underground openings of arbitrary shapes and fractures. Although the DD and FS methods have very different physical meanings, they are very similar in mathematical/numerical structure. With some modifications the following discussion can also be applied to the DD method. Similarly to the case for poroelasticity [120], a rigorous derivation of the indirect formulation for porothermoelasticity begins with Betti's reciprocal theorem to yield:

$$
\begin{gathered}
\sigma_{i j}(\mathbf{x}, t)=\int_{0}^{t} \int_{\Gamma}\left\{\sigma_{i j k}^{i p} \psi_{k}(\boldsymbol{\xi}, \tau)+\sigma_{i j}^{i s} \phi(\boldsymbol{\xi}, \tau)+\sigma_{i j}^{i h} \varphi(\boldsymbol{\xi}, \tau)\right\} d \Gamma(\boldsymbol{\xi}) d \tau \\
p(\mathbf{x}, t)=\int_{0}^{t} \int_{\Gamma}\left\{p_{i}^{i p} \psi_{i}(\boldsymbol{\xi}, \tau)+p^{i p} \phi(\boldsymbol{\xi}, \tau)+p^{i h} \varphi(\boldsymbol{\xi}, \tau)\right\} d \Gamma(\boldsymbol{\xi}) d \tau \\
T(\mathbf{x}, t)=\int_{0}^{t} \int_{\Gamma} \varphi T^{i h} d \Gamma(\chi) d \tau
\end{gathered}
$$

with:

$$
\begin{aligned}
\psi_{i} & =\left(\sigma_{i j}+\bar{\sigma}_{i j}\right) n_{j} \\
\phi & =-\left(v_{i}+\bar{v}_{i}\right) n_{i} \\
\varphi & =-\left(\bar{q}_{i}^{T}+q_{i}^{T}\right) n_{i}
\end{aligned}
$$

where the superscripts "ip", "ih", "is" denote the instantaneous force, heat source, and fluid source, respectively; and $n_{i}$ is the outward unit normal vector. The above set represents the integral equations for the porothermoelastic fictitious stress method. A similar set of expressions can be written for the displacement discontinuity method. The poroelastic fundamental solutions are readily available (e.g., [121]) and those for a heat source are listed in [118]. The integration of the latter over a straight line segment (assuming a constant distribution) is reported in [7].

Numerical Implementation The numerical procedure for porothermoelastic problems consists of solving the above integral equations for the unknown fictitious forces and fluid/heat sources. This is achieved using a boundary collocation technique, e.g., [122]. The problem boundary is divided into a number of elements $(J)$ and fictitious forces and fluid/heat sources are distributed over each element in space and time using

a desired shape function such that the sum of their effects satisfies the prescribed boundary conditions of e.g., tractions, pore pressures, and temperature. In the present implementation, it is assumed that boundary 
elements are straight segments with collocation points located at the center of each element. The induced stresses, pore pressures, and temperature on element " $M$ " due to a constant spatial distribution of continuous normal and shear forces and sources on element " $N$ ", occurring at time $\tau$, are given by:

$$
\begin{gathered}
\sigma_{n n}^{M}(\mathbf{x}, t)=\left[\sum_{N=1}^{J} \sigma_{n n k}^{c p}\left(\mathbf{x}, t^{\prime}\right) \Delta \psi_{k}^{N}+\sigma_{n n}^{c s}\left(\mathbf{x}, t^{\prime}\right) \Delta \phi^{N}+\sigma_{n n}^{c h}\left(\mathbf{x}, t^{\prime}\right) \Delta \varphi^{N}\right] \\
\sigma_{s n}^{M}(\mathbf{x}, t)=\left[\sum_{N=1}^{J} \sigma_{s n k}^{c p}\left(\mathbf{x}, t^{\prime}\right) \Delta \psi_{k}^{N}+\sigma_{s n}^{c s}\left(\mathbf{x}, t^{\prime}\right) \Delta \phi^{N}+\sigma_{s n}^{c h}\left(\mathbf{x}, t^{\prime}\right) \Delta \varphi^{N}\right] \\
p^{M}(\mathbf{x}, t)=\left[\sum_{N=1}^{J} p_{k}^{c p}\left(\mathbf{x}, t^{\prime}\right) \Delta \psi_{k}^{N}+p^{c s}\left(\mathbf{x}, t^{\prime}\right) \Delta \phi^{N}+p^{c h}\left(\mathbf{x}, t^{\prime}\right) \Delta \varphi^{N}\right] \\
T^{M}(\mathbf{x}, t)=\left[\sum_{N=1}^{J} T_{k}^{c h}\left(\mathbf{x}, t^{\prime}\right) \Delta \varphi_{k}^{N}\right]
\end{gathered}
$$

These equations state that at any given time, $\tau$, the stress and pore pressure at any point in the medium is the sum of all stresses and pore pressures caused by all the fictitious forces and sources taking place at time $t \leq \tau$ in the medium. The boundary influence coefficients such as $\sigma_{i j k}^{c p}$ denote the actual stresses (in the local system) at the midpoint of element $M$ due to a constant force applied to the element $N$ in the $k$ direction:

$$
\sigma_{i j k}^{c p}=a_{i \ell} a_{j m} \int_{0}^{t} \int_{\Gamma_{e}} \sigma_{\ell m k}^{c f} d \Gamma_{e} d t
$$

where the time integral is to be replaced by a discrete sum over the desired number of time steps in the solution procedure. The superscripts "cp", "cs" and "ch" refer to continuous force, continuous fluid source, and continuous fluid source, respectively; $a_{i \ell}$ is the rotation matrix from the influencing $(N)$ to the influenced $(M)$ element's coordinate system, and $t^{\prime}=t-\tau$. Note that the integration with respect to time is performed by tracing back to the time origin for the solution at any given time, i.e., it is carried out in the convolutional sense, so that no internal discretization would be necessary. The equation for the temperature is not coupled to the other sets and can be solved independently for the unknown hear sources. A computer program has been developed based on the above procedures. It utilizes analytical and numerical spatial integration of the fundamental solutions using a constant time step. 


\section{Appendix D}

\subsection{Publications}

In addition to 12 technical reports, the following publications have resulted from the project:

1) Koshelev, V. \& Ghassemi, A. 2003. Hydraulic fracture propagation near a natural discontinuity. 28th Workshop on Geothermal Reservoir Engineering, Stanford University, Stanford, CA, Jan. 27-29.

2) Koshelev, V. \& Ghassemi, A. 2003. Complex variable BEM for thermo- and poroelasticity. J. Eng. Anal. Boundary Elements (submitted).

3) Simakin, A. \& Ghassemi, A. 2003. Salt loaded heat pipes: steady-state operation and related heat and mass transport. J. Earth and Planetary Science (accepted for publication).

4) Ghassemi, A., Tarasovs, S. \& Cheng, A.-H. 2003. An Integral equation method for modeling threedimensional heat extraction from a fracture in hot dry rock. Int. J. Num. $\&$ Anal. Methods in Geomech. (in press).

5) Ghassemi, A. \& Zhang, Q. 2003. A transient indirect boundary element method for Poro-thermoelsaticity. BEM Technology 2003. Detroit.

6) Ghassemi A. \& Zhang, Q. 2002. Modeling fracture initiation \& propagation using an indirect porothermoelastic boundary element method. GRC Annual Conf. Reno.

7) Cheng, A. H.-D., Ghassemi, A. \& Detournay, E. 2001. A two-dimensional solution for heat extraction from a fracture in hot dry rock. Int. J. Numerical $\&$ Analytical Methods in Geomech., 25, 1327-1338.

8) Cheng, A. H.-D. \& Ghassemi, A. 2001. Effect of fluid leakoff on heat extraction from a fracture in hot dry rock. GRC 2001 Annual Meeting, San Diego, CA.

9) Ghassemi, A., Cheng, A. H.-D. \& Tarasovs, S. A three-dimensional solution for heat extraction from a fracture in hot dry rock using the boundary element method. Proc. 27th Workshop on Geothermal Reservoir Engineering, Stanford University, Stanford, California, January 28-30, 2002. 